\title{
PROTEIN AGGREGATION IN THE CYTOPLASM
}

\author{
Dissertation \\ for the award of the degree \\ "Doctor of Philosophy" Ph.D. Division of Mathematics and Natural Sciences \\ of the Georg-August-Universität Göttingen \\ within the doctoral program Biology \\ of the Georg-August University School of Science (GAUSS)
}

submitted by

Triana Amen

Göttingen, 2020 


\section{Thesis Committee}

Prof. Dr. Daniel Kaganovich

(Dept. of Experimental Neurodegeneration, University Medical Center Goettingen)

Prof. Dr. Tiago Outeiro

(Dept. of Experimental Neurodegeneration, University Medical Center Goettingen)

Prof. Dr. Gerhard Braus

(Dept. of Molecular Microbiology and Genetics, Institute for Microbiology and Genetics)

Members of the Examination Board

Reviewer: Prof. Dr. Daniel Kaganovich

(Dept. of Experimental Neurodegeneration, University Medical Center Goettingen)

Second Reviewer: Prof. Dr. Gerhard Braus

(Dept. of Molecular Microbiology and Genetics, Institute for Microbiology and Genetics)

Additional Reviewer: Prof. Dr. Jeffrey L. Brodsky

(Dept. of Biological Sciences, University of Pittsburgh)

Further members of the Examination Board:

Prof. Dr. Stefanie Pöggeler

(Dept. of Genetics of Eukaryotic Microorganisms, Institutes for Microbiology and Genetics)

Prof. Dr. Tiago Outeiro

(Dept. of Experimental Neurodegeneration, University Medical Center Goettingen)

Prof. Dr. Silvio O. Rizzoli

(Dept. of Neuro- and Sensory Physiology, University Medical Center Goettingen)

Prof. Dr. Kai Heimel

(Dept. of Molecular Microbiology and Genetics, Institute for Microbiology and Genetics)

Date of the oral examination: 30.04 .2020 
To my mother 


\section{Table of Contents}

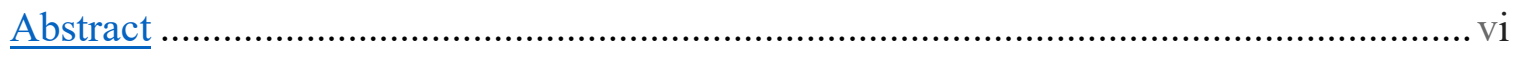

Acknowledgements ............................................................................................. vii

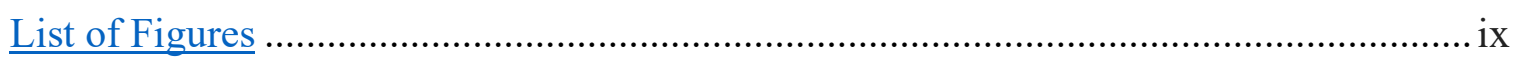

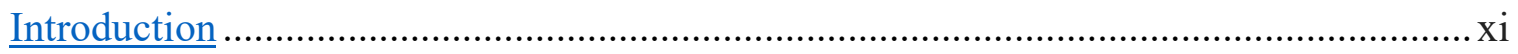

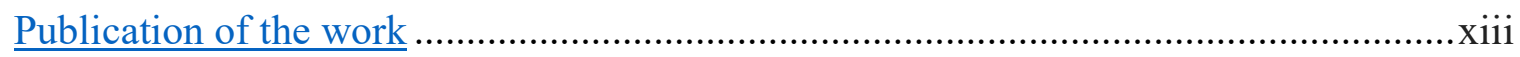

\section{$\underline{\text { CHAPTER } 1}$}

\section{Protein aggregation in the cytoplasm}

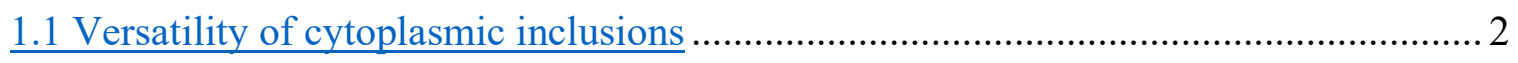

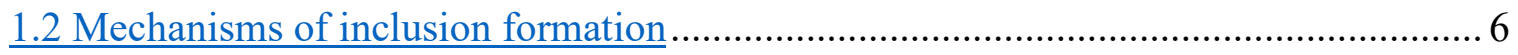

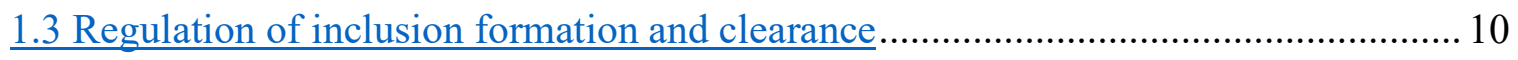

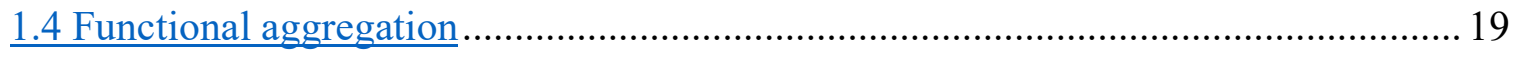

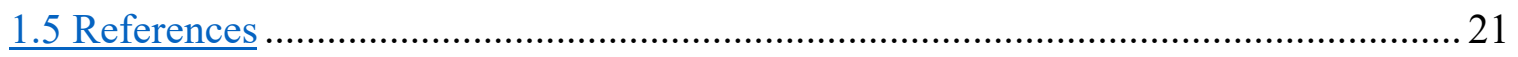

\section{CHAPTER 2}

\section{$\underline{\text { Stress Granule regulation of fatty acid oxidation }}$}

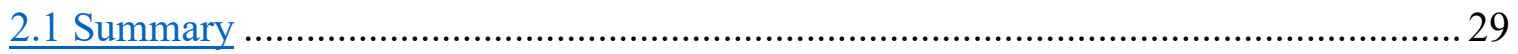

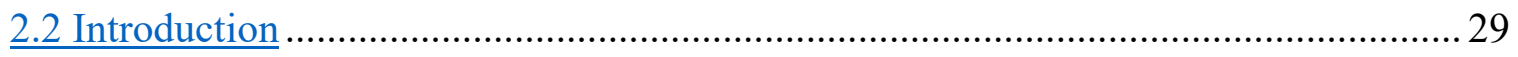

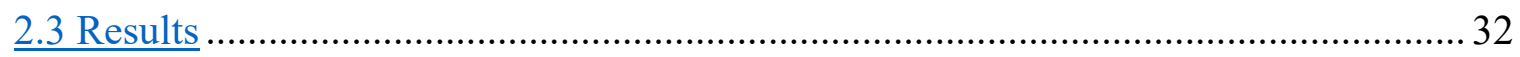

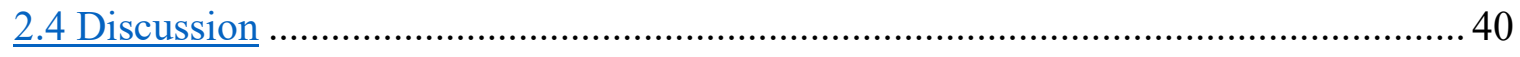

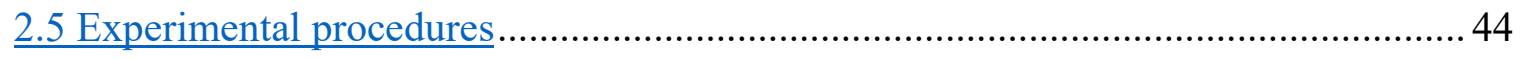

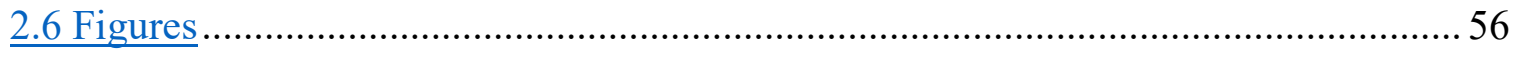

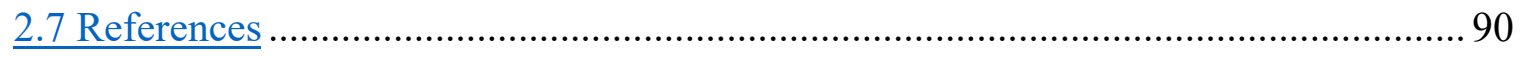




\section{CHAPTER 3}

$\underline{\text { Stress Granule mediate communication between stress response and lipid biogenesis }}$

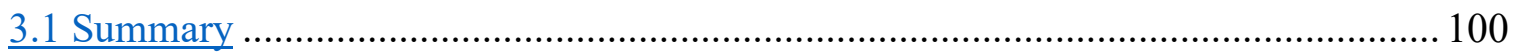

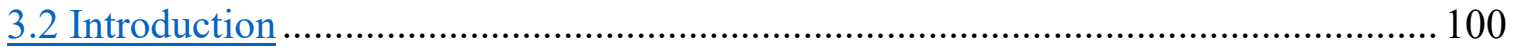

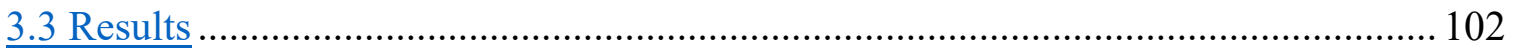

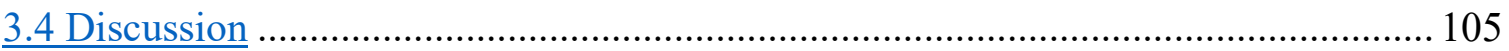

3.5 Figures

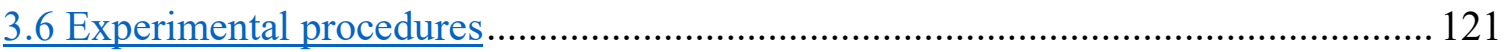

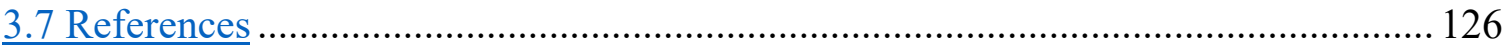

\section{CHAPTER 4}

Eisosome regulation of Stress granule formation in yeast Saccharomyces cerevisiae

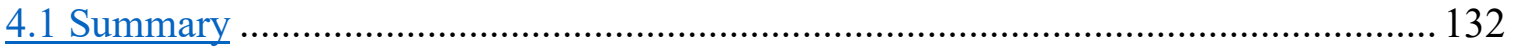

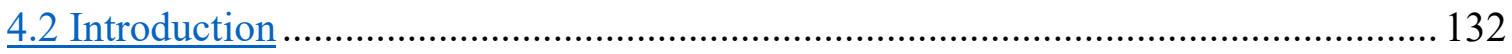

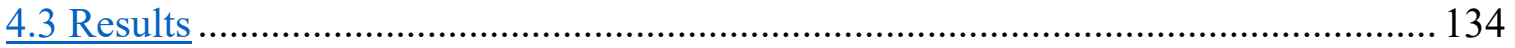

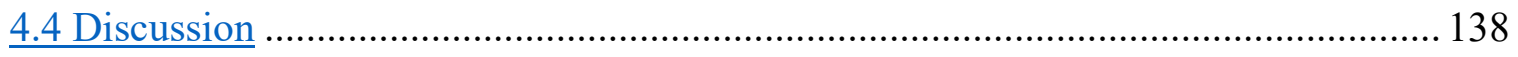

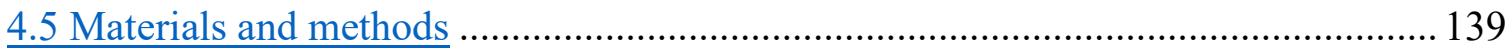

4.6 Figures

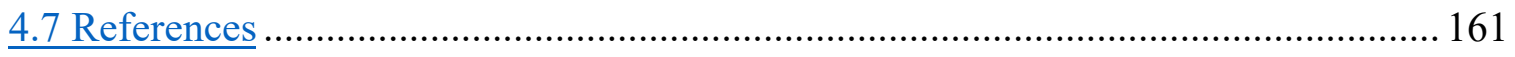

\section{CHAPTER 5}

\section{Conclusion and Future directions}

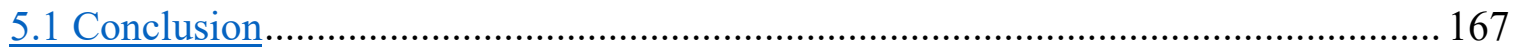

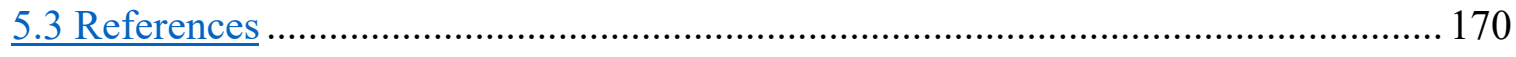




\begin{abstract}
The hypothesis motivating this thesis is that aggregation of proteins in the cytoplasm can have protective functions and can facilitate cell survival. Conversely, the disruption of functional aggregation can lead to cellular dysfunction and death. There is, therefore, a tremendous need to understand the mechanisms and precise sub-cellular architecture of functional aggregates. So far, the multitude of cellular aggregate structures or granules have been proposed to function as enzyme storage compartments, centers of memory retention, signaling hubs, mRNA triage compartments, degradation and protein refolding depots, structural elements, and transport granules. One of the unifying aspects of cytoplasmic granules appears to be stress dependent transient formation. In order to study cellular aggregation in live cells I chose to investigate factors that regulate the formation and clearance of cellular inclusions and their functions in cellular metabolism and signaling pathways. In this thesis I describe regulatory pathways that govern formation and function of granules with the focus on ribonucleoprotein granules, Stress Granules.
\end{abstract}




\section{Acknowledgements}

I have been fortunate to meet supporting, motivating, and diligent people who contributed to my thesis throughout the years. This work would not have been possible without my scientific adviser, Dr. Daniel Kaganovich, who devoted countless hours to help me gain technical knowledge and formulate my ideas. He taught me what quantitative cell biology is and what it is not.

I began work on this project in the Hebrew University of Jerusalem. Last two years of the work were conducted in the University Medical Center, Goettingen. I thank Jerusalem Brain Community of the Hebrew University for a generous financial support during my stay at the Hebrew University. I am grateful to the PhD GAUSS program at the University Medical Center for providing the stimulating research environment and teaching opportunities. I thank the members of the department of Experimental Neurodegeneration for their kindness, support, and creating an outstanding research environment.

I thank people who contributed to improving this work. I thank Prof. Daniel Kaganovich, Prof. Tiago Outeiro, Prof. Gerhard Braus, Prof. Maya Schuldiner, and Prof. Michael Glickman members of my thesis committee, former and present. I thank members of the Kaganovich Lab for the valuable discussions, and brainstorming ideas.

I received detailed technical support from the Hebrew University and University Medical Center facilities. I thank Dr. William Breuer, Prof. Christof Lenz, Lisa Neuenroth, and Thierry Wasselin for helping with the Mass Spectroscopy experiments, Christine Zellent and Prof. Jorg Wilting for helping with the transmission electron microscopy experiments, I thank Prof. Dr. Thomas Kneib for consultation on statistical analysis.

Several sections of this proposal would not be possible without collaborations which were done with many groups throughout the years. I thank collaborators and colleagues who shaped my views on the entire project while we were working on its parts. I thank Prof. Maya Schuldiner for her scientific leadership which is truly inspiring, Prof. Richard Gardner, for his experimental guidance and his kindness, Prof. Thomas Nystrom for his unique scientific approach, Prof. Sigal Ben Yehuda for an opportunity to work on bacterial nanotubes, Prof. Jeremy England for encouraging scientific curiosity, and Prof. Tiago Outeiro for providing both great advice on the 
scientific projects and valuable technical support. I also thank the members of their labs who contributed to the work.

Publication of the work wouldn't be possible without the joint effort of the editors and anonymous peers. I thank them for the effort and honest assessment of my work. This work was inspired by all the colleagues working in the field of protein aggregation and metabolic regulation.

Finally, I am immensely grateful to my mentor and scientific adviser Prof. Daniel Kaganovich. His scientific integrity and professional approach influenced how I see my scientific career beyond the $\mathrm{PhD}$. His unwavering support and endorsing of all my scientific endeavors allowed me to develop several projects described in this thesis. His patience, thorough guidance, and endless discussions took me through the hard parts of the projects. Dan is the greatest teacher I have ever encountered with deep understanding of the scientific conduct and unattainable standards in visualization of living systems. I am honored to be a part of the Kaganovich Lab. I thank Dan for believing in my abilities as a scientist, encouragement and support to explore new ideas, devoting time to explain, generosity, and innate kindness.

Goettingen, Germany

Triana Amen 


\section{List of Figures}

Figure 1.1 Spatial architecture of inclusions in yeast $S$. cerevisiae and mammalian cells. 4

Figure 1.2 Measuring Stress Granule dynamics............................................... 8

Figure 1.3 Elc1 regulates VHL inclusion formation in yeast $S$. cerevisiae...................... 11

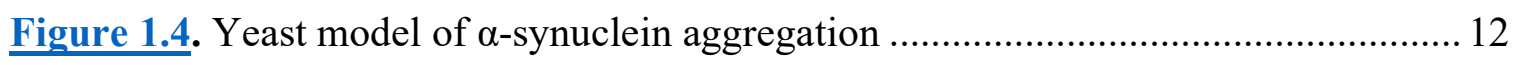

Figure 1.5 Vac17 regulates aggregate fusion and vacuole inheritance in yeast $S$. cerevisiae

Figure 1.6 Cyc8 inclusion formation during hyperosmotic stress is regulated by sumoylation

Figure 1.7 Endocytic trafficking of wild type (WT) and S129A mutant of human $\alpha$-synuclein in yeast $S$. cerevisiae. 16

Figure 1.8 Inclusions of human VHL are associated with Lipid Droplets in yeast S. cerevisiae 18

Figure 2.1 Stress Granule formation in starvation coincides with a decrease in fatty acid oxidation 75

Figure 2.2. Stress Granule formation promotes increased Fatty Acid import into lipid droplets 76

Figure 2.3 Stress Granules interactome reveals mitochondrial and Lipid Droplet association

Figure 2.4 VDAC undergoes reversible clustering during Stress Granule formation...... 78

Figure 2.5 VDAC2 is essential for mitochondrial influx of fatty acids 79

Figure 2.6 Stress Granule mediated signaling regulates VDAC clustering and fatty acid oxidation 80

Figure 2.7 Patient derived iPSC differentiated to neural precursors exhibit delayed FA Inhibition response 81 


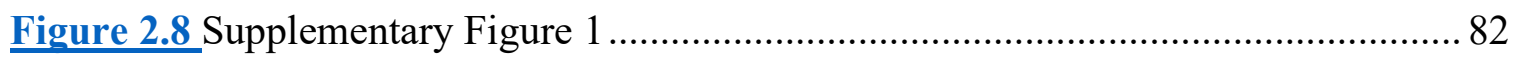

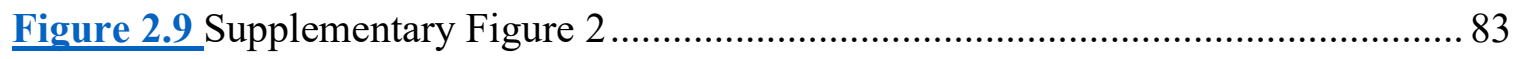

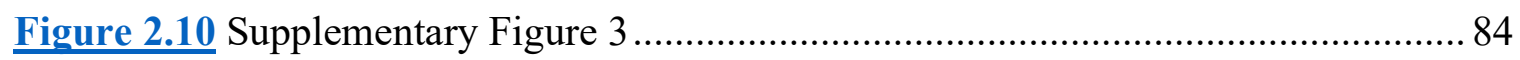

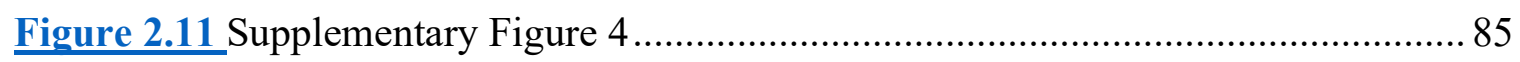

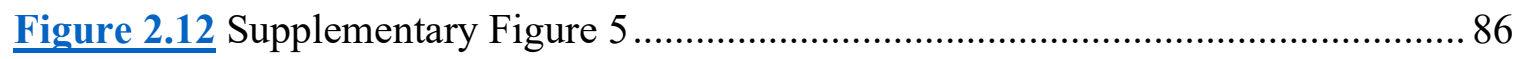

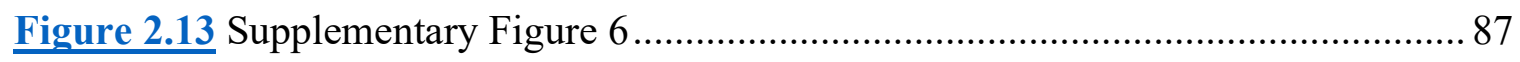

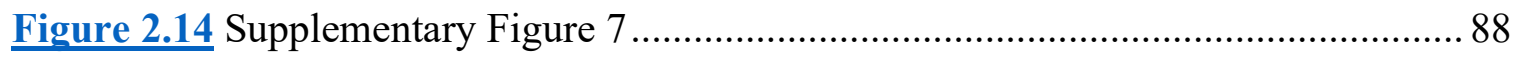

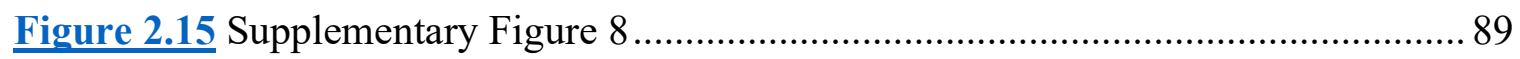

Figure 3.1 Small molecule inducers of Lipid Droplets also trigger Stress Granule formation

Figure 3.2 PPAR activation triggers Stress Granule formation ............................... 115

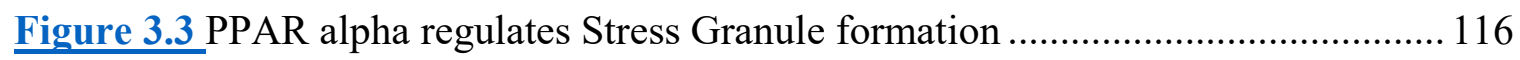

Figure 3.4 Stress Granules coordinate translation of PPAR induced transcripts ........... 117

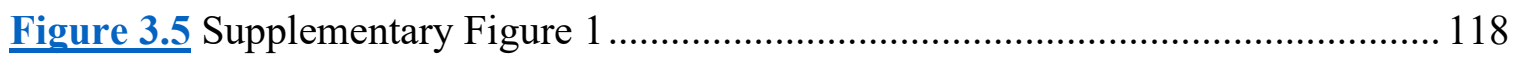

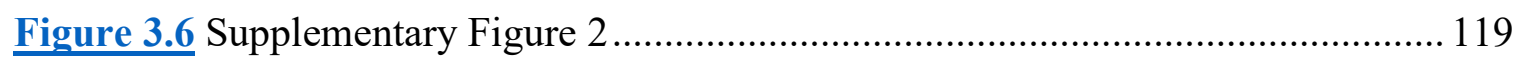

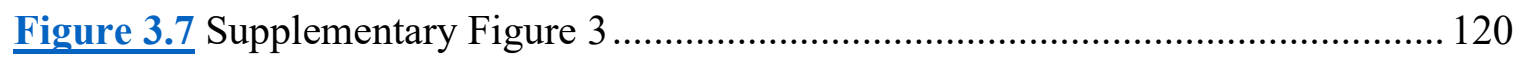

Figure 4.1 Stress granules associate with eisosomes during starvation ....................... 154

Figure 4.2 Pill undergoes Pkc1-dependent rearrangements during starvation .............. 155

Figure 4.3 $\mathrm{Pkc1}$ localizes to stress granules and is required for stress granule formation 156

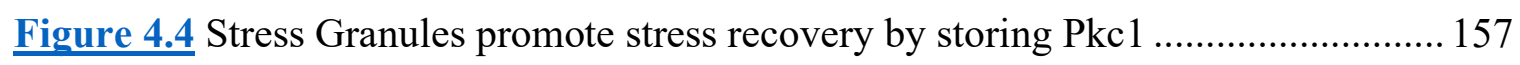

Figure 4.5 Stress granules associate with eisosomes during starvation ...................... 158

Figure 4.6 Eisosomes undergo Pkc1-dependent clustering during starvation................ 159

Figure 4.7 Pkc1 localizes to stress granules during starvation.................................. 160 


\section{Introduction}

My thesis describes the functions of protein inclusions that form in a living eukaryotic cell, with the emphasis on the cytoplasmic Stress Granules. The work presented here is done using human immortalized cell lines, human induced pluripotent stem cells, and yeast $S$. cerevisiae. Inclusions are often formed under stress conditions, thus where it was possible, I used naturally occurring stress conditions, such as nutrient starvation, to study inclusions in the physiological setting. Studying aggregation processes in the living cells requires caution, as over-production of inclusion components often triggers inclusion formation. To mitigate that, when working with live cells, I used endogenously tagged proteins. Throughout my thesis I interchange terms inclusion, protein aggregate, and granule, meaning a multi-protein cluster that forms during stress conditions often held together by multivalent non-specific interactions between proteins with disordered regions.

There are two defining aspects of functional protein inclusions: the relative transiency of their formation, and the ability to exert regulatory function that differ from the function of any of its individual components. Inclusions form under multitude of stress conditions and generally clear when normal condition resume. Inclusions often harbor dozens of different proteins, affecting multiple regulatory pathways in the cell. The first chapter is introductory, devoted to different mechanisms of inclusion formation and clearance, as well as their functions in the cell. I study different aspects of inclusion behavior in the cell with the unifying question being, what are the different mechanisms that allow cells to respond to stress conditions via protein clustering? In chapter 2 and following chapters I focus on Stress Granules. In chapter 2 I investigate the function of Stress Granules in the regulation of metabolic adaptation during starvation, specifically on the regulation of fatty acid oxidation metabolism during nutrient starvation stress. Chapter 3 extends the scope of Stress Granule regulation of lipid trafficking and transcriptional response in human cells.

Chapter 4 is on the formation and function of Stress Granules in yeast S. cerevisiae. I study how subcortical eisosome complex regulates the formation of Stress Granules and recruitment of protein kinase $\mathrm{C}$.

All of the ideas addressed in this thesis were developed throughout countless hours of discussion with my adviser Dr. Daniel Kaganovich. Chapter 1 is largely a result of multiple 
collaborations with the Labs of Prof. Richard Gardner, Prof. Jeremy England, Prof. Thomas Nystrom, Prof. Tiago Outeiro and Prof. Maya Schuldiner. 


\section{Publication of the work}

The experiments described in this dissertation were designed by me together with my scientific adviser Dr. Daniel Kaganovich. The experiments were performed by me.

Chapter 1 publications:

Triana Amen and Daniel Kaganovich Dynamic droplets: the role of cytoplasmic inclusions in stress, function, and disease Invited Review, Cellular and Molecular Life Sciences, Volume 72, Issue 3 (2015), Page 401-415

Sandra Malmgren Hill, Xinxin Hao, Johan Grönvall, Stephanie Spikings-Nordby, Per Widlnd, Triana Amen, Anna Jörhov, Rebcca Josfson, Daniel Kaganovich, Beidong Liu and Thomas Nyström Asymmetric Inheritance of Aggregated Proteins and Age Reset in Yeast Are Regulated by Vac17- Dependent Vacuolar Functions, Cell Reports, 16, 2016

Triana Amen, Diana F. Lázaro, Tiago F. Outeiro, Daniel Kaganovich Modeling neuronal pathology in yeast: insights into the molecular basis of Parkinson's disease, Israel Journal of Chemistry, Vol 55,2015

Michelle L. Oeser, Triana Amen, Cory M. Nadel, Amanda I. Bradley, Benjamin J. Reed, Ramon D. Jones, Janani Gopalan, Daniel Kaganovich, Richard G. Gardner Dynamic Sumoylation of a Conserved Transcription Corepressor Prevents Persistent Inclusion Formation during Hyperosmotic Stress, PLoS Genetics, 12(1), 2016.

Kelly P. Brock*, Ayelet-chen Abraham*, Triana Amen*, Daniel Kaganovich, and Jeremy L. England Structural Basis for Modulation of Quality Control Fate in a Marginally Stable Protein, Structure, Vol 23, 2015, * - co-author

Ofer Moldavski, Triana Amen, Smadar Levin-Zaidman, Miriam Eisenstein, Ilana Rogachev, Alexander Brandis, Daniel Kaganovich, * and Maya Schuldiner* Lipid Droplets Are Essential for Efficient Clearance of Cytosolic Inclusion Bodies, Developmental Cell, Vol 33, 2015, * - co-author Sandra Tenreiro, Madalena M. Reimão-Pinto, Pedro Antas, José Rino, Donata Wawrzycka, Diana Macedo, Rita Rosado-Ramos, Triana Amen, Meytal Waiss, Filipa Magalhães, Andreia Gomes, Cláudia N. Santos, Daniel Kaganovich and Tiago Fleming Outeiro Phosphorylation modulates clearance of alpha-synuclein inclusions in a yeast model of Parkinson's disease, PLoS Genetics, 2014

Chapter 2 manuscript has been submitted and is currently in revision in Cell: 
Triana Amen, Daniel Kaganovich Stress Granules regulate lipid metabolism by inhibiting mitochondrial influx of fatty acids, in revision (Cell)

Chapter 4 manuscript has been accepted in Science Signaling:

T. Amen, D. Kaganovich, Stress granules sense metabolic stress at the plasma membrane and potentiate recovery by storing active Pkc1. Sci. Signal. 13, eaaz6339 (2020), in press

Papers published during my $\mathrm{PhD}$ that are not discussed in the dissertation:

Triana Amen, Daniel Kaganovich Integrative modules for efficient genome engineering in yeast, Microbial Cell, Volume 4, Issue 6, 2017, Cover article

Gyanendra P. Dubey, Ganesh Babu Malli Mohan, Anna Dubrovsky, Triana Amen, Shai Tsipshtein, Alex Rouvinski, Alex Rosenberg, Daniel Kaganovich, Eilon Sherman, Ohad Medalia, and Sigal Ben-Yehuda Architecture and Characteristics of Bacterial Nanotubes, Developmental Cell, 36, 2016

Mikołaj Ogrodnik, Hanna Salmonowicz, Rachel Brown, Joanna Turkowska, Władysław Średniawa, Sundararaghavan Pattabiraman, Triana Amen, Ayelet-chen Abraham, Noam Eichler, Roman Lyakhovetsky and Daniel Kaganovich Dynamic JUNQ inclusions are asymmetrically inherited in mammalian cell lines through the asymmetric partitioning of vimentin, PNAS, 2014 


\section{Chapter 1}

\section{Protein aggregation in the cytoplasm}

Factors that affect protein aggregation span all levels of cellular complexity, from protein sequence and post translational modifications to membrane organelle association and regulation of cell division. In this chapter I discuss the mechanisms that govern the formation and clearance of protein inclusions in eukaryotic cells. We consider how inclusion formation is influenced by binding partners and post translational modifications of aggregated proteins, signaling cascades, organellar associations, and other pathways in the cell. Plethora of factors that influence protein aggregation reflect the functional diversity of protein inclusions. 


\subsection{Versatility of cytoplasmic inclusions}

Protein aggregation is an association of proteins into a high molecular weight cluster, often concomitant with a decrease in protein solubility and changes in protein conformation. Accumulation of proteins in aggregates has long been referred to as a pathological process. Aggregation is associated with cellular dysfunction, toxicity and disease progression (Bence et al., 2001; Vaquer-Alicea and Diamond, 2019). New findings, however, have led us to see aggregation process as a part of architectural changes during stress response, often serving protective functions. In recent years, we have come to appreciate the incredible complexity of protein granules in the cytoplasm of a living cell (Amen and Kaganovich, 2015; Kaganovich et al., 2008; Kedersha and Anderson, 2002; Pedley and Benkovic, 2017; Sagot et al., 2006). Inclusion formation is exploited by cells, allowing higher order regulation of cellular functions, such as protein storage and memory, signaling regulation and local response amplification, establishing polarity and filtering (Kaganovich, 2017). Protein inclusions, often referred to as membaneless organelles, granules, dynamic droplets, bodies, or simply aggregates, allow the cell to function during stress - sort the constant flow of misfolded proteins, store enzymes for later use, reconfigure signaling pathways, regulate translation, and nucleocytoplasmic transport, and govern replicative rejuvenation mechanisms (Amen and Kaganovich, 2015; Kaganovich et al., 2008; Kedersha and Anderson, 2002; Marshall and Vierstra, 2018; Ogrodnik et al., 2014; Petrovska et al., 2014; Thedieck et al., 2013; Zhang et al., 2018a). Thus, inclusion formation is emerging as an adaptive mechanism during physiological stress response. It is therefore unsurprising that pathological conditions that usually put cells under chronic stress are concomitant with the presence of aggregates in the cell. Neurodegenerative disorders, including Amyotrophic Lateral Sclerosis (ALS), Alzheimer's and Parkinson's diseases, are etiologically linked to pathological protein aggregation (Gundersen, 2010; Irvine et al., 2008; Jouanne et al., 2017; Li et al., 2013; Wolozin and Ivanov, 2019). Therefore, studying the mechanisms of aggregation will allow us to decipher the stress response pathways that led to the pathology in the first place.

Seminal discoveries on the misfolded protein inclusions and ribonucleoprotein (RNP) granules revealed the diversity of protein inclusions in the cells (Figure 1.1) (Bashkirov et al., 1997; Eystathioy et al., 2002; Ingelfinger et al., 2002; Johnston et al., 1998; Kaganovich et al., 2008; Kedersha and Anderson, 2002; van Dijk et al., 2002). In recent years, many new compartments were discovered, including metabolic enzyme storage compartments and protein quality control 
compartments designated for refolding or ubiquitination (De Graeve and Besse, 2018; Lelouard et al., 2004; Marshall and Vierstra, 2018; Pedley and Benkovic, 2017; Petrovska et al., 2014; Sagot et al., 2006; Spokoini et al., 2012; Szeto et al., 2006). Additionally, we learned that the protein composition of an inclusion is subject to a change depending on the stress conditions and formation mechanisms (Buchan et al., 2011). Interestingly, despite the breathtaking diversity of granules, they all exert only a few modes of regulation (Kaganovich, 2017). Inclusions can store proteins and RNA (including functional enzyme or proteins designated for degradation), amplify the signal by locally clustering functional proteins, and filter cellular components establishing polarity of cells or structures within the cell, combination of these principles and multiple components of the inclusions allows cells to regulate virtually all its functions via inclusion formation (Kaganovich, 2017). We still know relatively little about intra-organellar inclusions. However, it is likely that they follow the same principles proposed for cytoplasmic inclusions (Bruderek et al., 2018).

Although stress is a common denominator of inclusion formation, inclusions differ in their composition, localization, and functional modality in various stress conditions (Aulas et al., 2017; Weids et al., 2016). Misfolded, dysfunctional, or aggregation prone proteins are sequestered into protein quality control inclusions: JUNQ, IPOD, and Stress Foci (Figure 1.1) (Kaganovich et al., 2008; Spokoini et al., 2012). Formation of those inclusions is often triggered by acute or chronic protein folding stress, or overproduction of an aggregation prone protein (Spokoini et al., 2012). Cell attempts to fold or recover the dysfunctional protein using elaborate chaperone network, and if that is not possible, it is either designated for proteasomal degradation using the Ubiquitinproteasome system (JUNQ) or packed in a highly dense aggregated compartment (IPOD), possibly for eventual lysosomal degradation (Gidalevitz et al., 2011; Hill et al., 2016; Kaganovich et al., 2008). Acute stress leads to the formation of triage compartments (Stress Foci) that can be fused to the IPOD or refolded by chaperones (Alberti, 2012; Hill et al., 2016; Shiber et al., 2013; Spokoini et al., 2012). Mislocalization of misfolded proteins to a non-native inclusion compartment creates a highly toxic environment for the cell, implying that formation of protein quality control inclusions is a way to mitigate stress from dysfunctional proteins (Weisberg et al., 2012). Thus, inclusions of misfolded protein are protein quality control compartments which maintain protein homeostasis (Alberti, 2012; Kaganovich et al., 2008).

Another example of functional aggregation is formation of Storage Granules. Several proteins, including metabolic enzymes, proteasome subunits, and actin, form bodies or storage 
compartments during stress (Marshall and Vierstra, 2018; Pedley and Benkovic, 2017; Petrovska et al., 2014; Sagot et al., 2006). Those compartments preserve the functional proteins for later use, for example non-dividing and starving yeast cells store actin in bodies, creating an immediately available pool of proteins to jump start cellular functioning after normal conditions resume (Marshall and Vierstra, 2018; Sagot et al., 2006). Aggregation in this case creates a fitness advantage for the cell (Pedley and Benkovic, 2017).
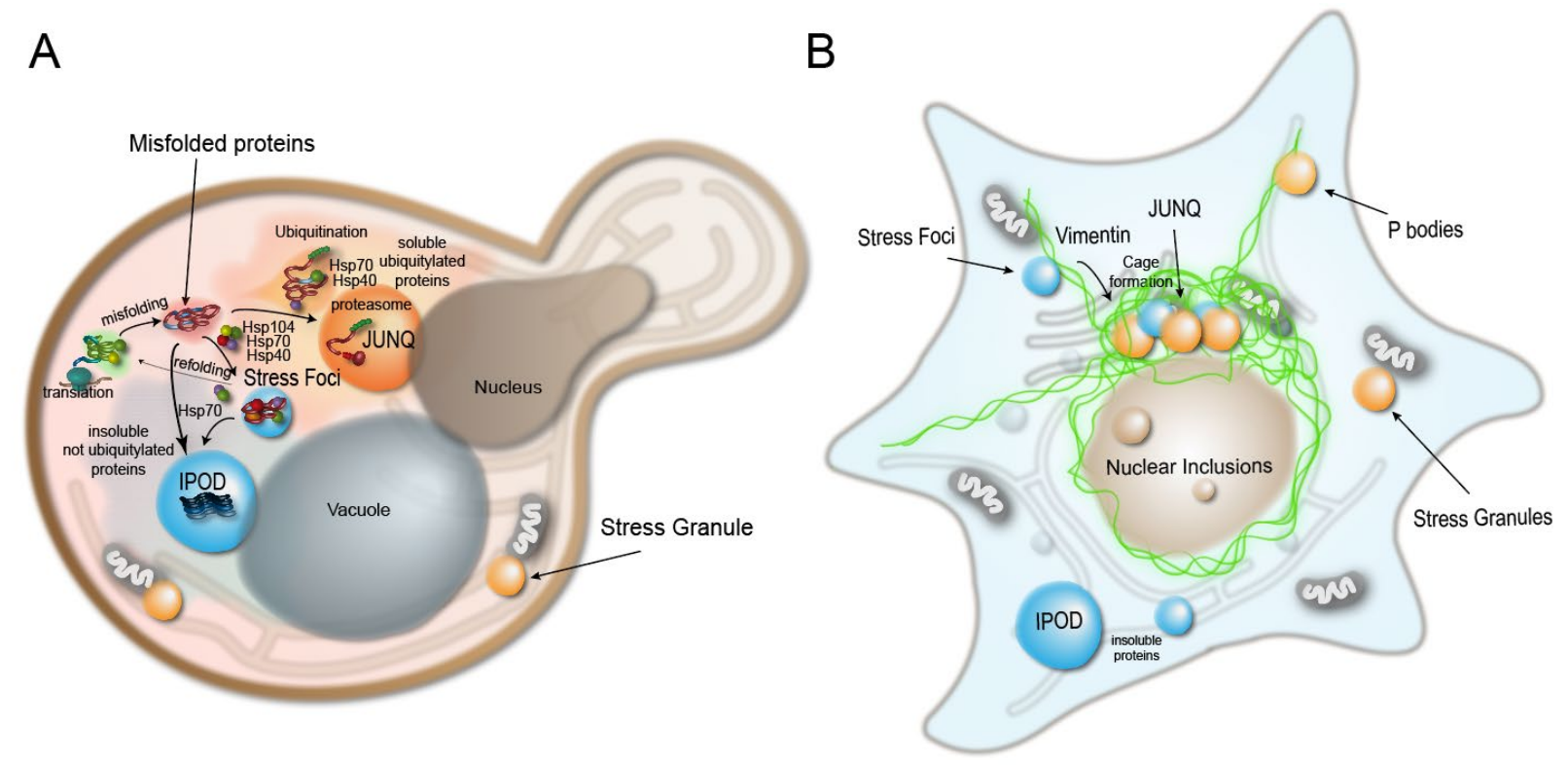

Figure 1.1. Spatial Architecture of inclusions in yeast S. cerevisiae (A) and mammalian cells (B). Misfolded proteins are partitioned into nuclei-associated JUNQ compartments, and cytoplasmic IPOD compartments. The process is regulated by the chaperone network, protein concentrations, post translational modifications, and cellular architecture. Acute stress conditions result in the formation of Stress Foci, where misfolded proteins are transiently retained. Stress Foci can merge with the IPOD, for insoluble aggregation or targeted to the JUNQ for eventual proteasomal degradation. Stress Granules and P bodies are ribonucleoprotein (RNP) granules that harbor stalled translation pre-initiation complexes, RNA-binding proteins, and cell signaling components. RNP granules regulate multiple cellular functions, and can be contaminated by aggregated misfolded proteins, which will result in the aberrant aggregation of their components.

One of the most striking examples of functional aggregation is formation of the ribonucleoprotein granules (RNP granules). Those granules contain dozens of different proteins 
and mRNAs and are able to regulate diverse cellular functions, such as memory storage, nucleocytoplasmic transport, apoptosis, kinase signaling, translation and mRNA triage (Arimoto et al., 2008; Kedersha and Anderson, 2007; Thedieck et al., 2013; Zhang et al., 2018a; Zhang et al., 2018b). Stress Granules (SGs), RNP granules that contain stalled pre-initiation complexes, RNA-binding proteins, such as TDP43, FUS, and kinases, has been thought of as a signaling hub of the mammalian cell during stress conditions (Buchan and Parker, 2009; Kedersha et al., 2013; Kedersha et al., 2005). Key signaling molecules, like protein kinase C, and mTOR kinase relocate to the SG compartment and affect its formation (Fournier et al., 2013; Jevtov et al., 2015; Kobayashi et al., 2012; Sfakianos et al., 2018; Thedieck et al., 2013). SG appear in response to many physiological stresses, like heat, oxidative stress, starvation, cold shock, and have been shown to prevent apoptosis and reduce production of reactive oxidative species (Arimoto et al., 2008; Takahashi et al., 2013; Thedieck et al., 2013), serving a protective function.

Granules are often referred to as membraneless organelles (Gomes and Shorter, 2019). Unlike most membrane compartments, granule formation is often conditional. Inclusions usually form during stress conditions and are generally cleared when normal conditions resume (Protter and Parker, 2016; Spokoini et al., 2012). Cells exploit aggregate formation and clearance mechanisms to regulate availability and activity of proteins without the need to re-synthesize and degrade molecules (Oeser et al., 2016; Petrovska et al., 2014). Control over aggregation allows cell to regulate virtually all its functions, from enzyme availability to memory and replicative rejuvenation (Kaganovich, 2017; Ogrodnik et al., 2014; Petrovska et al., 2014; Si and Kandel, 2016). Pathology involving protein aggregation often stems from a disruption of the mechanisms of inclusions formation, clearance or is indicative of a chronic stress, forcing cell to respond by sequestering proteins inside granules. For example, aberrant persistent aggregation of SG is implicated in the pathology of Amyotrophic Lateral Sclerosis (ALS), an age-related neurodegenerative disorder, and it is concomitant with the death of motor neurons (Li et al., 2013; Wolozin and Ivanov, 2019). Thus, studying inclusion clearance mechanisms along with inclusion formation can provide invaluable insights into the mechanisms of cellular pathology. 


\subsection{Mechanisms of protein aggregation}

Historically, aggregation was associated with protein misfolding, or loss of native conformation (Fink, 1998). Protein synthesis or translation is usually accompanied by establishing a native 3D structure by the amino acid sequence. During and after the translation proteins are accompanied by chaperone machinery that, together with the cytosol, facilitate establishing of the protein's native conformation (McClellan et al., 2005b). Misfolded or non-native protein states are prone to aggregation, due to exposed hydrophobic regions which tend to associate, creating protein clusters (Fink, 1998). Therefore, translation and folding process is a constant source of aggregation intermediates, which comprise not only newly arising polypeptides, but also prematurely terminated sequences, such as Defective ribosome products (DRiPS) (McClellan et al., 2005b; Yewdell et al., 1996). To mitigate the constant accumulation of misfolded peptide chains and to keep proteins in a soluble state, cell has to continuously reverse the aggregation process or degrade misfolded proteins (Gidalevitz et al., 2011; McClellan et al., 2005b). For that cell utilizes the chaperone network and the ubiquitin proteasome system (UPS). Chaperones bind misfolded regions stabilizing them from hydrophobic association, and UPS is designated for protein degradation (Hershko and Ciechanover, 1998; McClellan et al., 2005b). The two system work together to establish a balance of protein production and degradation in order to maintain protein homeostasis - steady state of production of functional proteins and destruction of damaged and dysfunctional proteins in the cell (Gidalevitz et al., 2011). Exceeding the capacity of the degradation or chaperone system leads to overload of misfolded proteins which primes widespread aggregation. For example, recombinant expression of model misfolded proteins above critical concentrations or components or RNP granules lead to the formation of inclusion compartments (Gilks et al., 2004; Spokoini et al., 2012). Cells have several distinct compartments designated for storage, destruction, or refolding of the misfolded proteins (Kaganovich et al., 2008; Lelouard et al., 2004; Spokoini et al., 2012; Szeto et al., 2006). For example, acute heat stress in yeast $S$. cerevisiae, leads to the formation of Stress Foci, multiple dynamic inclusions, marked by chaperone Hsp104 (Spokoini et al., 2012). If stress is chronic, those compartments fuse and form an insoluble protein deposit (IPOD) near the vacuole (Hill et al., 2016; Kaganovich et al., 2008), when stress is resolved the proteins will be refolded or released to a cytoplasmic soluble pool (Spokoini et al., 2012). The IPOD formation is regulated by vacuole inheritance adaptor protein Vac17, which facilitate fusion of inclusions on their way to the vacuole-bound compartment (Hill 
et al., 2016). Additionally, Hsp104 binds endomembrane trafficking components suggesting that vesicle trafficking can facilitate inclusion formation (Hill et al., 2016). Thus, chaperones that recognize and bind misfolded proteins govern the formation of the inclusion.

Mutations, irreversible protein modification, stresses all lead to protein misfolding and the formation of protein quality control compartments (Du et al., 2015; Fink, 1998; Spokoini et al., 2012). However, the bulk of what we call inclusions aren't formed due to misfolding events. To add a level of complexity, in recent years it has become clear that proteins have stretches of disordered regions which do not obtain a defined conformation (Deiana et al., 2019). At a certain level of intrinsic disorder protein is not able to establish any native conformation while still being functional. These proteins are classified as intrinsically disordered protein (IDP) (Forman-Kay and Mittag, 2013; Oldfield and Dunker, 2014) and constitute 30 to 50\% of human proteome (Deiana et al., 2019; Forman-Kay and Mittag, 2013). IDPs thought to be hubs in protein interaction networks due to their capacity to bind multiple partners (Dunker et al., 2005; Oldfield and Dunker, 2014) and regulators of cellular signaling (Wright and Dyson, 2015). Not surprisingly, IDPs play central role in the formation of RNP granules, such as Stress Granules, P granules, nucleolus, and other non-membrane organelles (Elbaum-Garfinkle et al., 2015; Feric et al., 2016; Kato et al., 2012; Weber and Brangwynne, 2012). The aggregation of RNP granules can be explained by phase separation of proteins and RNA in cytoplasmic solutions, governed by multi-valent interactions of its components (Boeynaems et al., 2018; Kato et al., 2012; Weber and Brangwynne, 2012). Interesting characteristic of these aggregates is that they are dynamic in nature, exhibiting interchange of components within the aggregate and with its surroundings. These inclusions are often referred to as liquid droplets (Brangwynne et al., 2009), due to an intermixing of components and droplet like behavior in the cellular context (Figure 1.2). The phase separated granules can acquire gel-like, liquid, or solid states, generally exhibiting slow diffusion of proteins within the inclusion (Figure 1.2) (Boeynaems et al., 2018; Kaganovich, 2017; Kato et al., 2012; Weber and Brangwynne, 2012). High cytoplasmic concentrations of proteins and other macromolecules, referred to as macromolecular crowding, facilitates phase separation (Ellis, 2001; Kaur et al., 2019). Formation of granules can be a way to stabilize protein concentrations, by aggregating the excess. Aggregation in this case can be seen as a dosage compensation mechanism. For example, aggregation of proteins maintains the stoichiometry of protein complexes in aneuploidy conditions (Brennan et al., 2019; Saarikangas and Barral, 2016). 
A

B
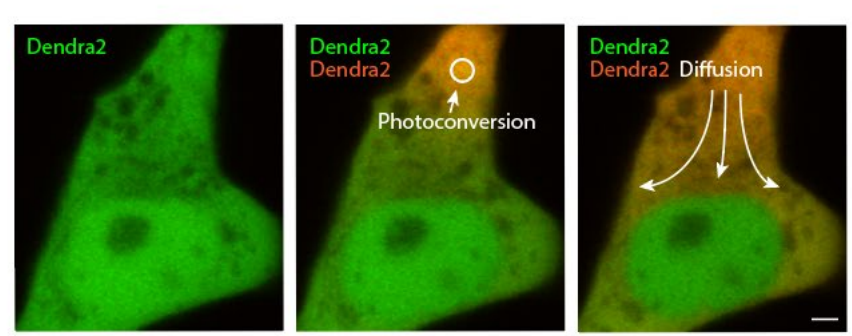

C
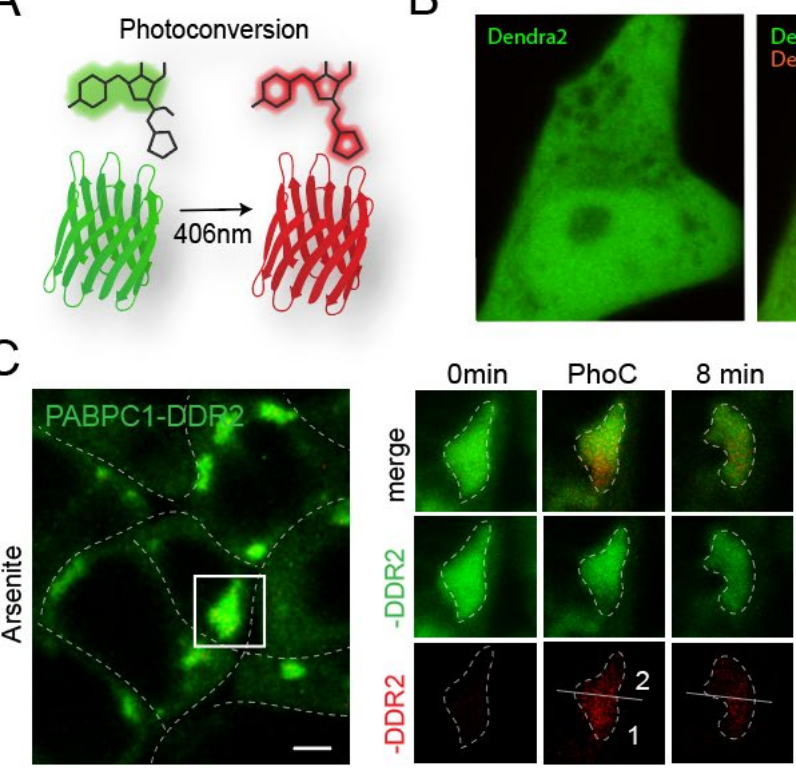

D
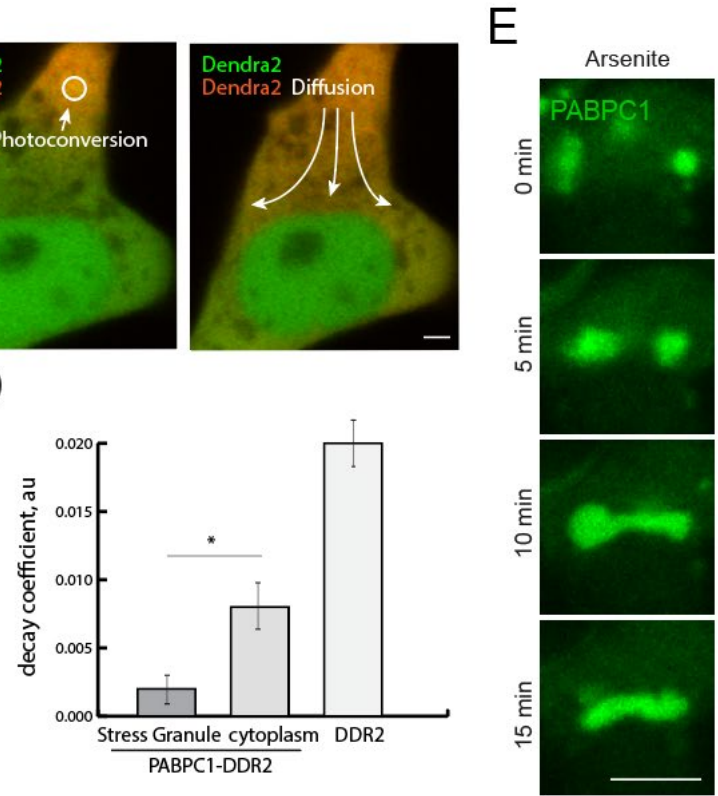

Figure 1.2. Measuring Stress Granule dynamics (A) Schematic of photoconversion (B) Confocal microscopy of photoconversion of over expressed Dendra2 (DDR2) protein in human cells, scale bar $1 \mu \mathrm{m}$ (C) Confocal microscopy of SG photoconversion in HEK293T cells, expressing CRISPR/Cas9 tagged PABPC1-DDR2 (D) Quantification shows decay coefficient (extracted from fitted exponential decay function) of red fluorescence intensity in a photoconverted area of SG compartment, or overexpressed DDR2, $\mathrm{p}<0.05, \mathrm{n}=10$ (E) Confocal microscopy of SG fusion in HEK293T cells expressing PABPC1-DDR2 treated with arsenite for 45 minutes prior to the start of the experiment, scale bar $5 \mu \mathrm{m}$

Phase separation as a mechanism of granule formation became a widely recognized phenomena, explaining how cell establishes polarity, creates subcompartments in the nucleus, and regulates signaling (Boeynaems et al., 2018; Elbaum-Garfinkle et al., 2015; Shin and Brangwynne, 2017; Weber and Brangwynne, 2012). The mechanisms behind phase transitions mostly stem from a multivalent interaction of proteins - here IDPs come into play, because it is easier for them to bind multiple partners creating oligomeric states that can be further organized into high molecular weight clusters (Boeynaems et al., 2018; Wright and Dyson, 2015). Disordered proteins involved in the phase transitions have repetitive sequences, sometimes referred to as low complexity domains, LCDs, and lack hydrophobic stretches that are crucial for the folding of ordered proteins (Dyson and Wright, 2005; Franzmann and Alberti, 2019; Wootton, 1994). Disordered and low complexity regions are able to establish a wide array of conformations with multiple potential 
interactors (Boeynaems et al., 2018; Franzmann and Alberti, 2019; Kato et al., 2012). These proteins are often RNA binding proteins, and RNA plays a pivotal role in the formation of membraneless organelles such as SGs (Elbaum-Garfinkle et al., 2015; Kato et al., 2012; Weber and Brangwynne, 2012).

The molecular mechanisms that initiate phase separation vary, often involving stress inducedchanges in signaling and post-translational modifications, or variations in the concentration of RNA and salts (Boeynaems et al., 2018; Elbaum-Garfinkle et al., 2015; Schuster et al., 2018; Shin and Brangwynne, 2017). P granule, a perinuclear RNP granule specific to the germline in Caenorabditis elegans, is formed through phase separation of LAF-1, DDX3 RNA-helicase (Elbaum-Garfinkle et al., 2015; Wang and Seydoux, 2014) Intrinsically disordered domain of LAF-1 is necessary and sufficient to initiate viscosity transitions, depending on the temperature and salt concentration (Elbaum-Garfinkle et al., 2015; Schuster et al., 2018). Unlike P granules, formation of SGs has been thought to depend on multiple mechanisms. SGs are non-membrane ribonucleoprotein compartments that form transiently during stress in most of the cells ranging from yeast to mammals (Buchan and Parker, 2009; Kedersha and Anderson, 2002). The initial stage of SG formation includes phosphorylation of eukaryotic translation initiation factor 2 alpha, eIF2alpha, or inhibition of translation by dephosphorylation of 4EBP1, both events decrease bulk translation rates and initiate polysome disassembly (Anderson and Kedersha, 2002; Clemens, 2001; Fingar et al., 2004; Gingras et al., 1999; Sfakianos et al., 2018; Wu et al., 2017). Hence, drugs that stabilize polysomes, such as cycloheximide, are able to inhibit SG formation (Kedersha et al., 2000). The basic SG constituents are components of disassembled polysomes, 48S translation preinitiation complexes, mRNA binding proteins, and translation regulators (Kedersha and Anderson, 2002). TIA-1, mRNA binding protein that shuttles between nucleus and the cytoplasm, is inducing SG formation depending on its concentration by assembly through glutamine-rich prion-related domain (Gilks et al., 2004). Downstream of translation inhibition SG assembly is governed by post translational modification of several proteins. It is thought that endoribonucleases G3BP1 and G3BP2 (G3BP) nucleate Stress Granules, dependent on their phosphorylation status (Panas et al., 2019). G3BP nucleation step is essential for SG formation via translation inhibition mechanisms (Yang et al., 2019). G3BP binds several proteins, including USP10 and Caprin1, that regulate SG assembly through competitive G3BP complex formation (Kedersha et al., 2016b). The model proposed by Kedersha et al., 2016 posits that Caprin1-G3BP 
complexes promote SG assembly, while USP10-G3BP complexes inhibit SG assembly (Kedersha et al., 2016a). However, G3BP mutant which is unable to bind USP10 or Caprin1 is still able to form SGs (Kedersha et al., 2016b). In addition, SGs compartment composition and formation is regulated by multiple kinase pathways (Aditi et al., 2019; Heberle et al., 2019; Jevtov et al., 2015; Kobayashi et al., 2012), allowing cells to tune the compartment composition in response to different stresses (Buchan et al., 2011). It is becoming clear that control over the composition and phase of inclusions is critical for cellular functioning (Dewey et al., 2012; Mateju et al., 2017; Patel et al., 2015).

\subsection{Regulation of inclusion formation and clearance}

To gain the full functionality of an inclusion cell must exert control over its formation, phase transitions, composition, and clearance. Inclusion formation depends on the protein sequence, availability of binding factors, chaperone machinery, post translational modifications, stress conditions, organelle associations to name a few (Aulas et al., 2017; Brock et al., 2015; Buchan et al., 2011; Hill et al., 2016; Tenreiro et al., 2014). Phase separated inclusions often contain multi valent IDPs, with repeats of low complexity regions, allowing them to bind multiple proteins, which drives polymerization (Banani et al., 2016; Schuster et al., 2018). Tuning the valency of such proteins manually, for example increasing the arginine-rich RNA-binding repeats of LAF-1 protein, which organizes the $\mathrm{P}$ granules in $\mathrm{C}$. elegans, results in a regulated formation of the granule (Elbaum-Garfinkle et al., 2015; Schuster et al., 2018). In the cellular context, increase in the local concentration of repeated disordered domains or increase in RNA concentration results in phase transitions (Elbaum-Garfinkle et al., 2015; Gilks et al., 2004). Thus, cells can exert control over phase transitions by tuning transcription and translation rates. Additionally, many RNP granule components are shuttling between the nucleus and the cytosol, thus regulating nucleocytoplasmic transport modulates phase transitions (Gilbertson et al., 2018; Guo et al., 2018).

Aggregation, especially in case of misfolded proteins, involves chaperones, which disaggregate, refold, and accompany proteins in the inclusions (Alberti, 2012; McClellan et al., 2005b). Chaperoning is an energy consuming process (Shorter, 2011; Shorter and Southworth, 2019). Degradation of proteins is also energetically expensive (Goldberg, 2003; Peth et al., 2013). During stress resources become scarce, and it often results in a widespread aggregation of proteins (Pu et al., 2019; Saad et al., 2017). ATP availability acts both as a potentiator for protein 
solubilization by chaperones, and also has been shown to solubilize aggregates directly, destabilizing aggregated proteins (Hayes et al., 2018; Patel et al., 2017). Therefore, cell can regulate aggregation metabolically, producing more energy. This can explain the correlation between aging-associated metabolic decline and widespread aggregation (David et al., 2010; Henry, 2000).

Availability of binding partners, other than chaperones, affect protein stability and inclusion formation (Figure 1.3) (Brock et al., 2015; Schoenfeld et al., 2000). VHL, von HippelLindau tumor suppressor, binds Elongin $\mathrm{C}$ and other partner forming a E3 ubiquitin ligase (Schoenfeld et al., 2000). Otherwise rapidly degraded by proteasome, VHL is stabilized by Elongin B and C interactions (Kamura et al., 2002; Schoenfeld et al., 2000). Recombinant expression of human von Hippel-Lindau tumor suppressor VHL in yeast $S$. cerevisiae has long been used to study stress induced aggregation of misfolded proteins (Kaganovich et al., 2008; McClellan et al., 2005a). VHL lacking all of its binding partners is prone to form aggregates under heat stress conditions when expressed recombinantly in yeast (Kaganovich et al., 2008; Spokoini et al., 2012). Interestingly, deletion of $E L C 1$, orthologous elongin gene in yeast, results in inclusion formation of recombinant human VHL in normal condition, indicating that stabilization of VHL prevents its recruitment to inclusions (Figure 1.2C). Therefore cells, can regulate inclusion formation by binding partner availability.
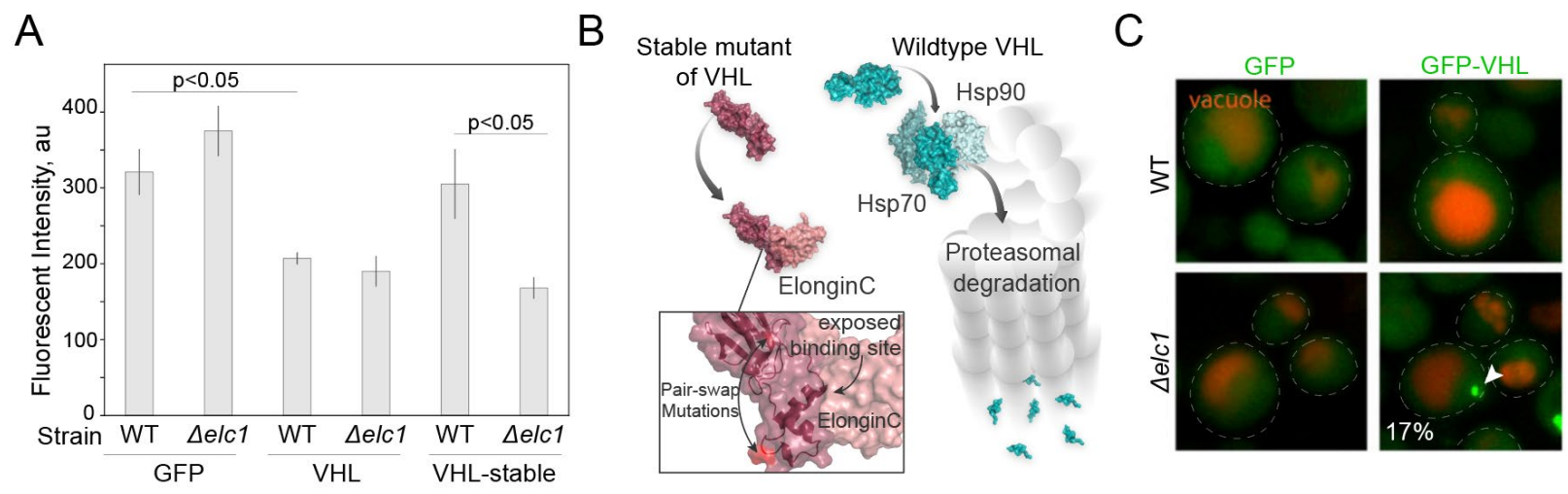

Figure 1.3 Elc1 regulates VHL inclusion formation in yeast $S$. cerevisiae. (A) Fluorescent intensity of GFP, GFP-VHL, and GFP-VHL-stable after stop of translation in wildtype (WT) and ELC1 deletion strains, inducible GAL1 promoter was used to drive the expression. Graph shows mean \pm standard deviation, $\mathrm{n}=30$ (B) Schematic of preferential routes of human VHL expressed in yeast (Jones and Gardner, 2015). Wildtype 
VHL is guided by chaperones to proteasome, where it gets degraded, stabilization of VHL-Elongin C binding by pair-swap mutation results in longer protein life (C) Elongin C binding to VHL regulates inclusion formation. Representative confocal images of WT and ELCl deletion strains expressing GFP, GFP-VHL are shown, scale bar $1 \mu \mathrm{m}$. Number in the right lower panel indicates percentage of cells with aggregates.

It is becoming clear that inclusion formation is modulated by intracellular membranes and membrane trafficking inside the cells (Gorbenko and Trusova, 2011; Lee et al., 2020; Mogk and Bukau, 2014). Aggregation can be enhanced by crowding of proteins on the membrane, increased local concentration, or acquiring of an aggregation prone conformation due to the membrane binding (Gorbenko and Trusova, 2011). For example, recombinant expression of a membrane bound aggregation prone protein, alpha-synuclein (Figure 1.3A) (Outeiro and Lindquist, 2003), results in inclusion formation, which is concomitant with endocytic vesicle aggregation (Figure 1.4, 1.7) (Sancenon et al., 2012; Soper et al., 2008). Modulation of vesicle trafficking is inherently linked to both aggregation and toxicity of alpha synuclein (Sancenon et al., 2012; Tenreiro et al., 2014).

A

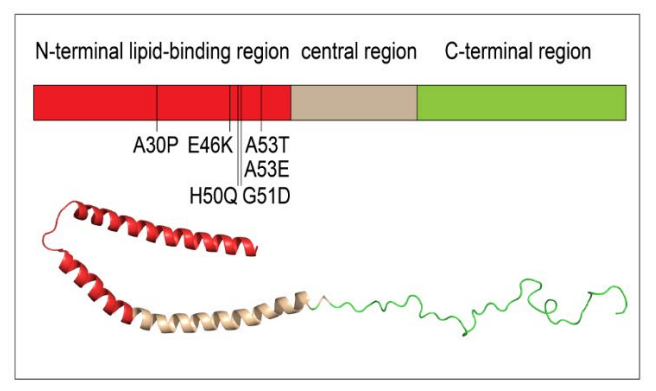

C

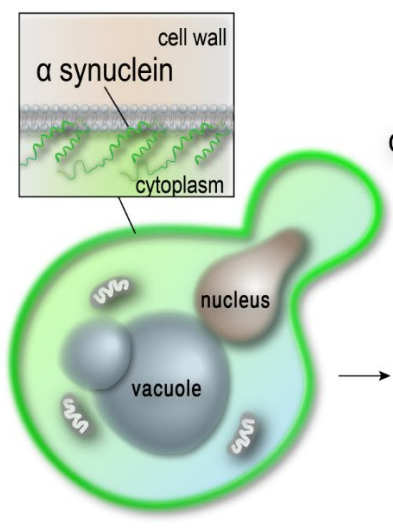

B
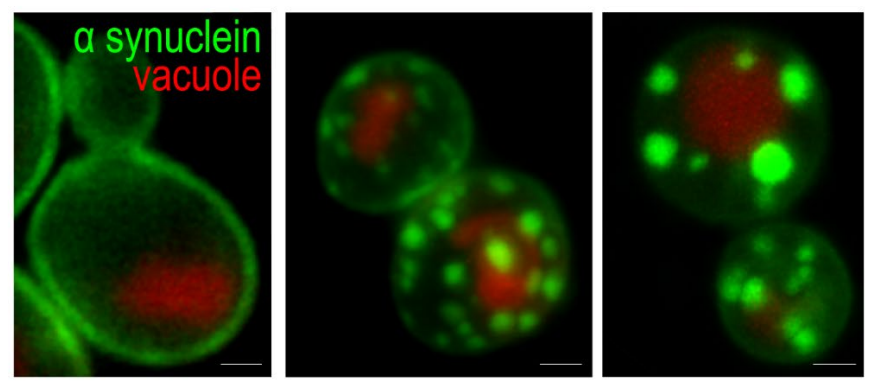
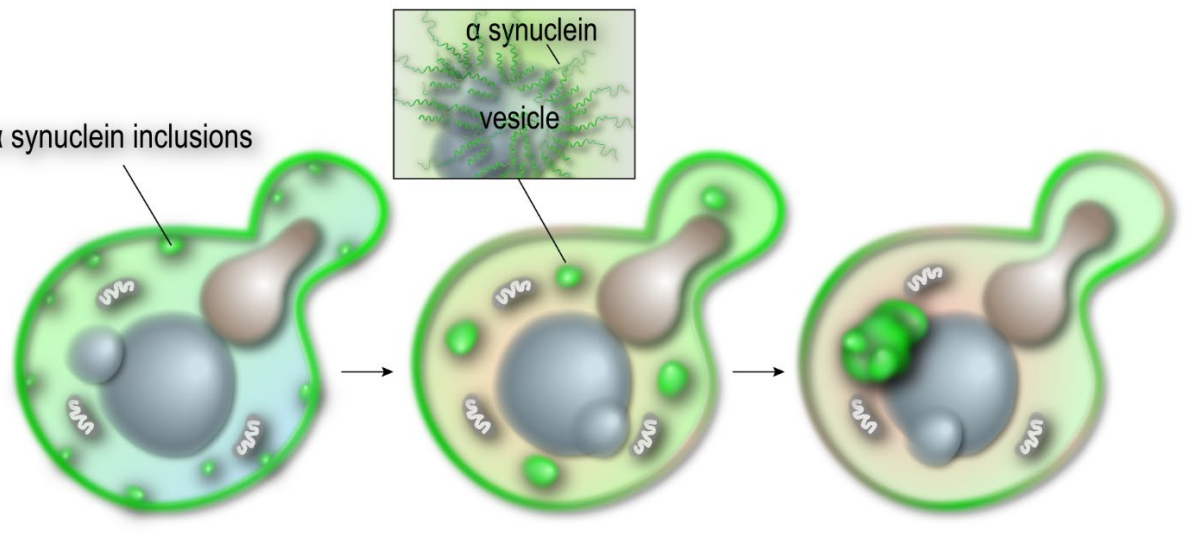
Figure 1.4. Yeast model of $\alpha$-synuclein aggregation (A) Protein domains and known mutations of human $\alpha$-synuclein (Ulmer et al., 2005) (B) Membrane and inclusion localization of $\alpha$-synuclein fused to GFP in yeast $S$. cerevisiae. Confocal images are shown scale bar $1 \mu \mathrm{m}$ (C) Schematic of $\alpha$-synuclein aggregation in yeast $S$. cerevisiae

Membrane trafficking in yeast $S$. cerevisiae affects the formation of IPOD and Stress Foci protein quality control compartments (Figure 1.5) (Hill et al., 2016). Deletion of the vacuole inheritance regulator, $V A C 17$, results in a decreased rate of Stress Foci fusion towards vacuoletethered IPOD compartment, and over expression facilitates the fusion (Hill et al., 2016; Kaganovich et al., 2008; Spokoini et al., 2012).
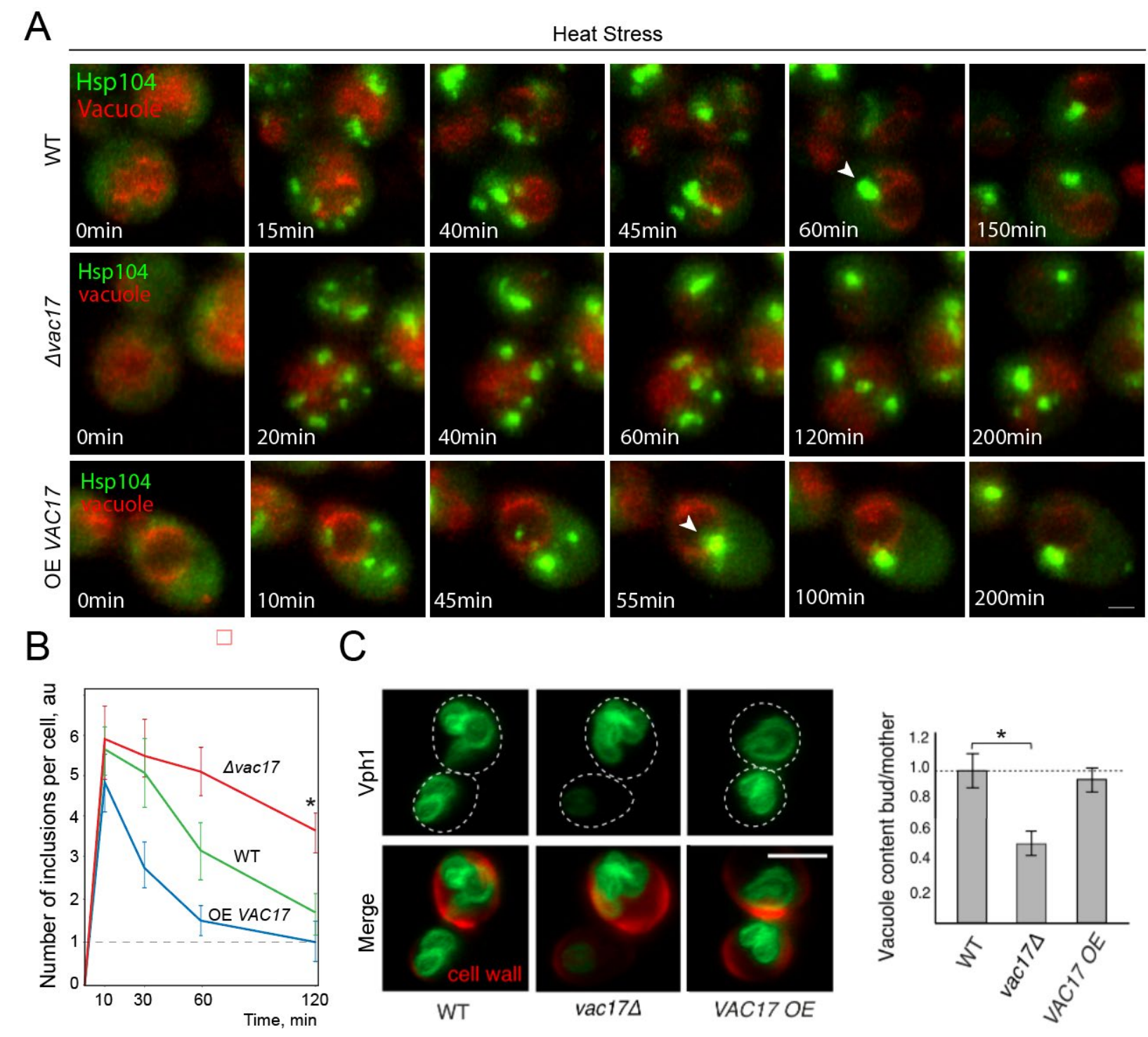
Figure 1.5 Vac17 regulates aggregate fusion and vacuole inheritance in yeast S. cerevisiae (A-B) Vac17 regulate IPOD formation. Cells expressing endogenously tagged Hsp104-GFP were subjected to $38^{\circ} \mathrm{C}$ heat stress for 200 minutes. Confocal images were taken every 5 minutes, representative confocal frames are shown, scale bar $1 \mu \mathrm{m}$. Vacuole is stained with FM4-64. Arrow heads indicate IPOD formation. Graph shows number of inclusions per cell in the population, mean $\pm \mathrm{SEM}, \mathrm{n}=30$, * indicates $\mathrm{p}<0.05$ (C) $\operatorname{Vac} 17$ regulates vacuole inheritance. Cells expressing endogenously tagged Vph1-GFP were grown in complete media. Representative frames of dividing cells are shown, scale bar $5 \mu \mathrm{m}$. Cell wall was stained with calcofluor white prior to the imaging. Graph shows fluorescent intensity of the vacuole content in the dividing cells $(\mathrm{au})$, mean $\pm \mathrm{SEM}, \mathrm{n}=30$, * indicates $\mathrm{p}<0.05$.

Post translational modifications play central role in the formation and clearance of the inclusions. Protein aggregation and degradation is tightly linked to its ubiquitination (Goldberg, 2003) Poly-ubiquitinated proteins are generally targeted to the UPS for degradation, chaperones, however, can recruit tagged proteins to inclusions where proteins await degradation or refolding (Goldberg, 2003; Lecker et al., 2006; Shiber et al., 2013; Szeto et al., 2006). Scarce resources or aberrant levels of chaperones result in persistent inclusions of ubiquitinated proteins (Shiber et al., 2013; Szeto et al., 2006). Modification of proteins with a small ubiquitin-related modifier, SUMO, results in reduced aggregation propensity (Krumova et al., 2011; Kuo et al., 2014; Oeser et al., 2016). In yeast $S$. cerevisiae, Tup1 and Cyc 8 constitute a transcriptional co-repressor (Tzamarias and Struhl, 1994), which forms inclusions during hyperosmotic stress, releasing repressed transcription of genes involved in osmotic stress response (Figure 1.6A,B) (Oeser et al., 2016; Tzamarias and Struhl, 1994). Cyc8 inclusions typically clear within one hour of stress (Figure 1.6C), dependent on the sumoylation of the inclusion components (Figure 1.6). Preventing sumoylation, using a quadruple lysine to arginine replacement mutant of Cyc8, results in persistent cytoplasmic and nuclear inclusions (Figure 1.6) (Oeser et al., 2016).

Hyperphosphorylation of proteins is often implicated in pathological protein aggregation, for example hyperphosphorylation of microtubule binding protein TAU is a hallmark of Alzheimer's disease (Andorfer et al., 2003; Park et al., 2018). Interestingly, presence of polyphosphates destabilizes proteins and promote their aggregation (Sasahara et al., 2019; Zhang et al., 2019). Phosphorylation of both eIF2alpha and G3BP plays central role in the formation of SG compartments (McInerney et al., 2005; Reineke et al., 2017). However, phosphorylation can also 


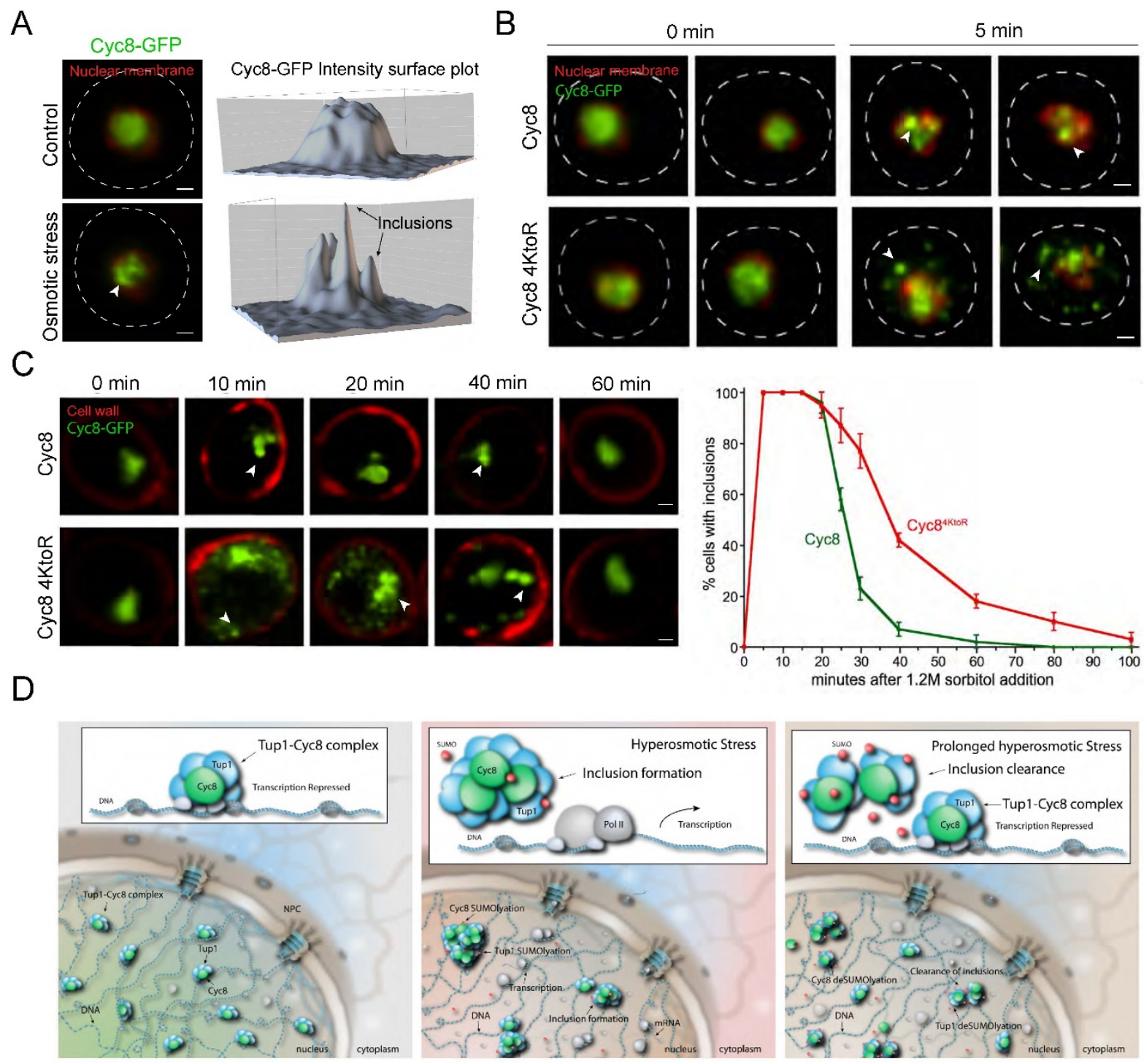

Figure 1.6 Cyc8 inclusion formation during hyperosmotic stress is regulated by sumoylation (A) Cyc8 forms nuclear inclusions during hyperosmotic stress. Cells were grown to mid log phase and treated with $1.2 \mathrm{M}$ sorbitol for 5 minutes, nuclear membrane marker - Nup53-mCherry. Representative Structured Illumination Microscopy (SIM) images and intensity surface plots are shown, scale bar $1 \mu \mathrm{m}$. Arrowhead indicate nuclear inclusions (B) Sumoylation mutant of Cyc8 forms cytoplasmic inclusions during hyperosmotic stress. Cells lacking Cyc8, complemented with wildtype or 4KtoR mutant of Cyc8 were treated with $1.2 \mathrm{M}$ sorbitol for 5 minutes and visualized with SIM. Representative images are shown, scale bar $1 \mu \mathrm{m}$. Arrowheads indicate inclusions. (C) Sumoylation of Cyc8 regulates inclusion clearance. Cells complemented with wildtype or $4 \mathrm{KtoR}$ mutant Cyc8 were subjected to 60 minutes of $1.2 \mathrm{M}$ sorbitol. Representative SIM images show the time course of inclusion formation and clearance, scale bar $1 \mu \mathrm{m}$. Cell wall marker - calcofluor white. Graph shows percentage of the cells with inclusions, mean \pm SEM, $n=30$ 
(D) Schematic of Cyc8 inclusion formation during hyperosmotic stress. Tup1-Cyc8 inclusion formation leads to transcription inhibition. Sumoylation of the inclusions aids in clearance and restores the transcription.

promote inclusion clearance. Phosphorylation of apha-synuclein on $129^{\text {Ser }}$ promotes vesicle trafficking to the vacuole inducing aggregate degradation (Figure 1.7) (Petroi et al., 2012; Tenreiro et al., 2014) in the yeast model of alpha-synuclein aggregation (Outeiro and Lindquist, 2003).

A
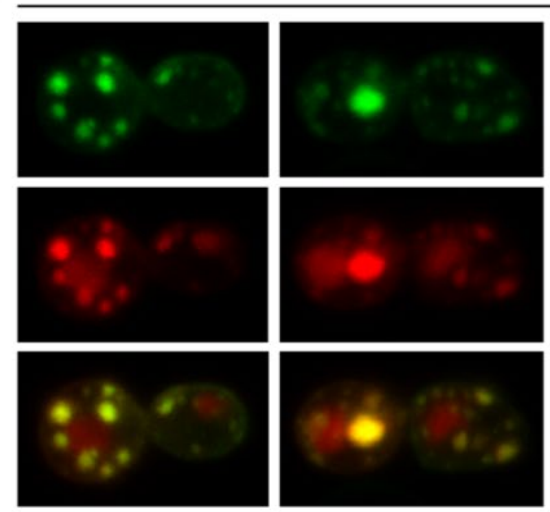

B
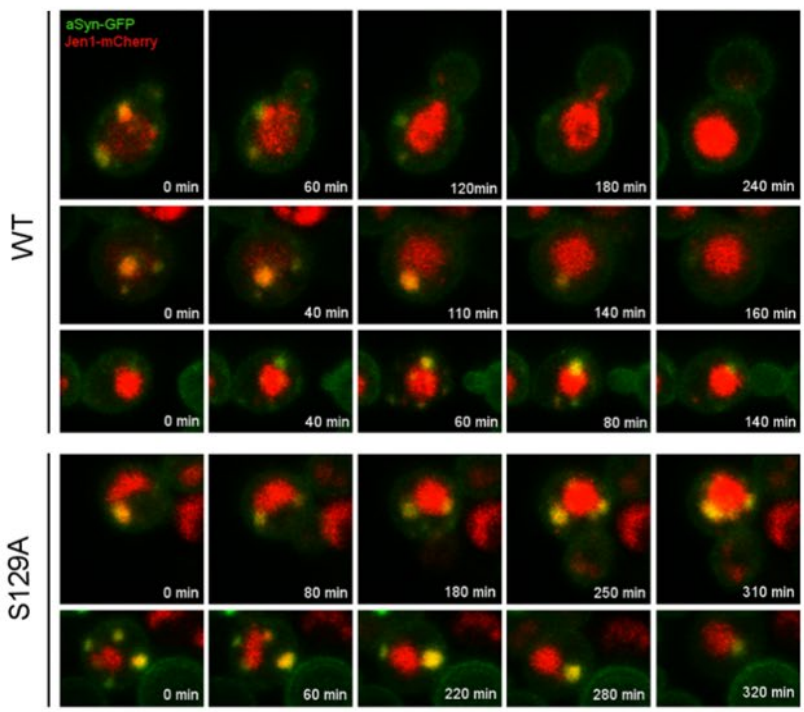

S129A
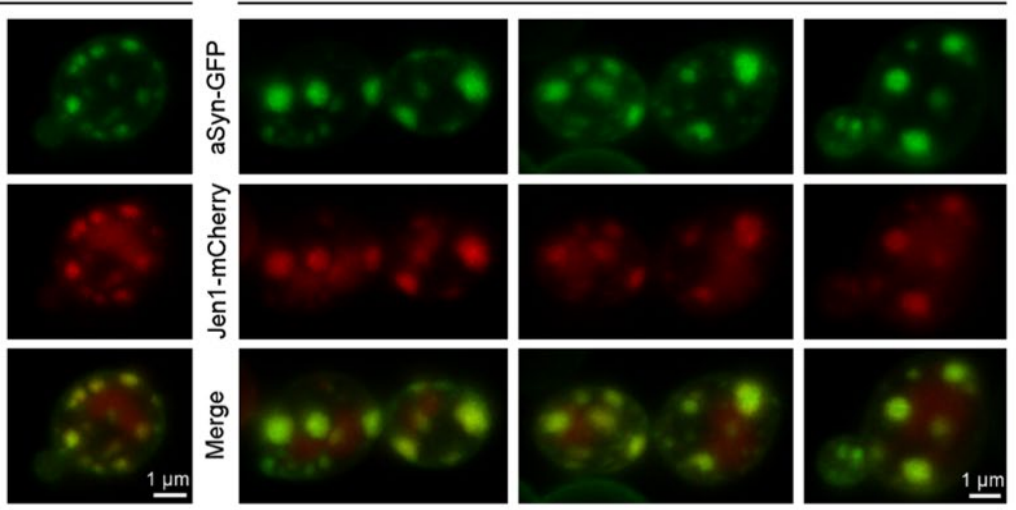

C

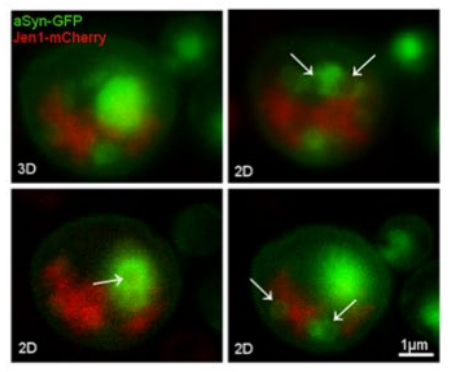

Figure 1.7 Endocytic trafficking of wild type (WT) and S129A mutant of human $\alpha$-synuclein (aSyn) in yeast $S$. cerevisiae (A) $\alpha$-synuclein inclusions co-localize with endosomal compartments. Yeast cells expressing endocytosis markers Jen 1-mCherry and WT or S129A $\alpha$-synuclein fused to GPP were grown to $\log$ phase in a complete media, JEN1-mCherry expression was driven by GAL1 promoter and induced 
by galactose addition. Representative confocal images are shown. (B) S129A $\alpha$-synuclein mutant impairs vesicle fusion with the vacuole. Representative confocal frames from a time-lapse are shown. (C) $\alpha$ synuclein clusters with the membrane compartments. Confocal images showing 3D reconstruction and 2D planes are shown. (A) aSyn inclusions localize to membrane and endosomal compartments. Cells coexpressing Jen1-mCherry and WT or S129A aSyn-GFP were grown at $30^{\circ} \mathrm{C}$ in glucose and shifted to galactose for 6 hours. Jen1-mCherry is constitutively synthesized, trafficked to the membrane, and reabsorbed through the endocytic pathway, eventually ending up in the vacuole. Since mCherry is $\mathrm{pH}$ stable it is visible in the vacuole. aSyn-GFP inclusions colocalize with Jen1-mCherry in endosome compartments throughout the entire endocytic pathway. (B) aSyn foci cluster with vesicles. Images shown are still frames from a time-lapse series showing internalization of aSyn through the endocytic pathway. In some cases of low level of aSyn aggregation enters the vacuole. As the aggregation level increases, however, aSyn-containing vesicles are trapped, likely in late endosomes. (C) aSyn inclusions are present as vesicles or vesicle clusters (arrows). Expression of S129A aSyn-GFP was induced for 8 hours. 3D reconstruction and single $\mathrm{z}$ sections (2D) are shown. Each z series was acquired with 0.4micron step size and 21 total steps.

Inclusion clearance can result from its gradual degradation via autophagy or UPS or solubilization of proteins (Buchan et al., 2013; Kaganovich et al., 2008; Lamark and Johansen, 2012; Spokoini et al., 2012). In the case of alpha-synuclein recombinant expression in yeast, clusters of aggregated vesicles are destroyed by autophagy (Petroi et al., 2012). Misfolded protein compartments, such as Stress Foci, can be refolded by chaperones, resulting in inclusion clearance (Spokoini et al., 2012). Interestingly, inclusion clearance can be regulated by lipids. Inclusions of misfolded proteins in yeast $S$. cerevisiae exhibit lipid droplet association and form in close proximity to lipid droplets (Figure 1.8) (Moldavski et al., 2015). Lipid droplets store lipids in the form of sterols or triacylglycerides. Sterol deficient strains were not able to clear stress-induced inclusion, implying that lipids play role in protein solubilization (Moldavski et al., 2015).

Inclusion exhibit stress-dependent composition (Aulas et al., 2017; Buchan et al., 2011). Regulation of inclusion composition in case of phase separated inclusions is dependent on the concentrations and stoichiometries of inclusion scaffolding proteins, which can change over time, resulting in recruitment of different proteins to the inclusions (Banani et al., 2016). 


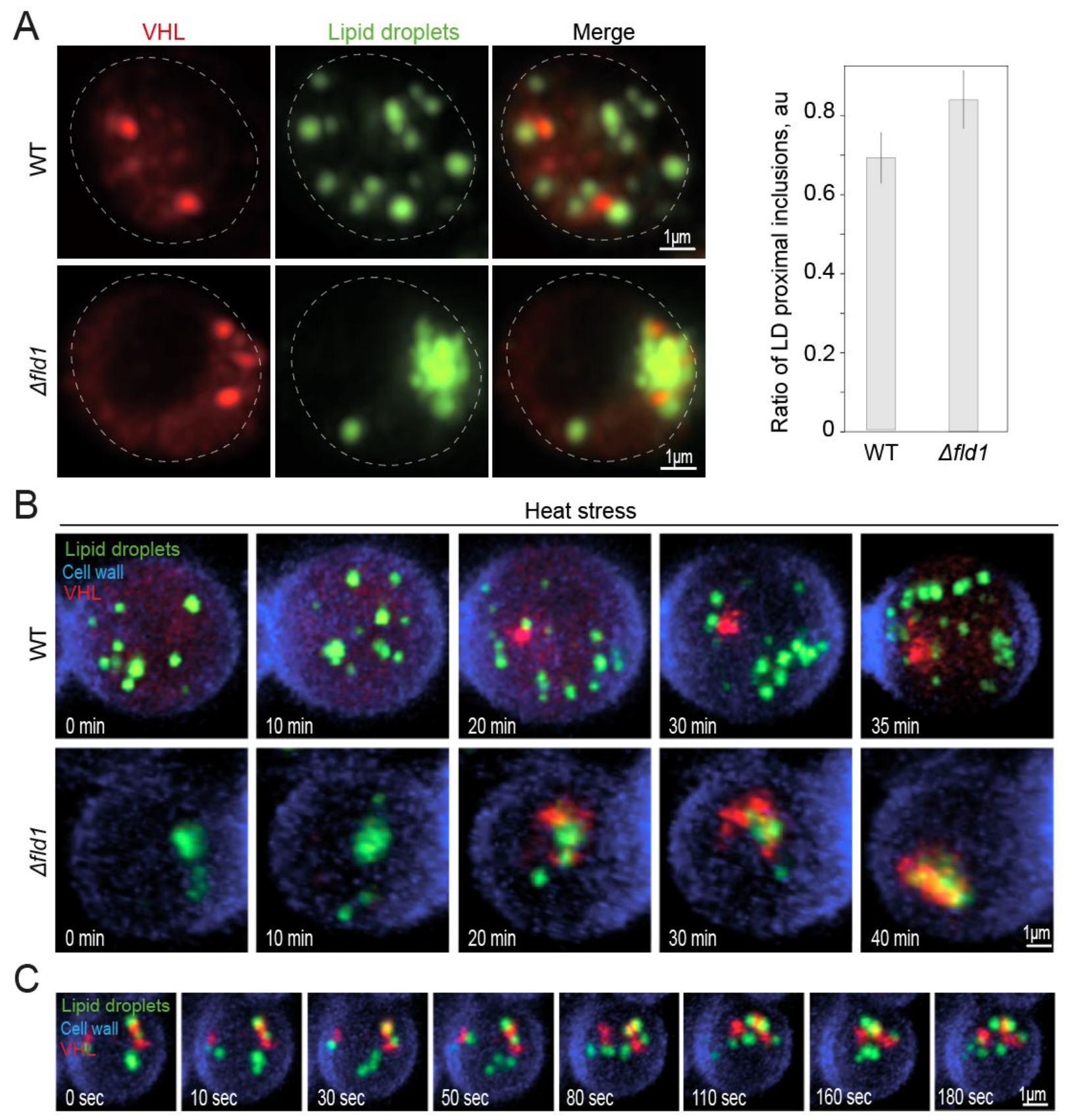

Figure 1.8 Inclusions of human VHL are associated with Lipid Droplets in yeast S. cerevisiae (A) Proximity of LD and VHL inclusions. WT and $\Delta f l d 1$ yeast expressing mCherry-VHL were subjected to heat stress to form inclusions and visualized with Structures Illumination microscopy (SIM/TIRF). Lipid Droplets were stained with Bodipy $(1 \mu \mathrm{M})$ prior to the imaging. Graph shows ratio of proximal inclusions, mean \pm SEM, $n=30$. (B) Time-lapse of inclusion formation during heat stress showing association with LDs. WT and $\Delta f l d l$ yeast expressing mCherry-VHL were subjected to heat stress for 60 minutes. Representative confocal frames are shown. (C) Inclusions track together with LDs during movement in the 
cytoplasm. Time-lapse tracks LD-inclusion clusters for 3 minutes. Representative confocal frames are shown.

\subsection{Functional aggregation}

Inclusions can contain up to hundreds of different constituents. Portion of them are regulators of inclusion formation, often referred to as scaffolding proteins (Banani et al., 2016), the rest of the composition differs and reflects the functionality of the inclusion (Aulas et al., 2017; Buchan et al., 2011). One of the first realizations of aggregate functionality arised from studying prion inheritance in yeast $S$. cerevisiae (Tyedmers et al., 2008). Prions are proteins that are able to selfpropagate its conformation, resulting in formation of amyloid or highly aggregated fibrils, responsible for human neurodegenerative diseases, transmissible spongiform encephalopathies (Aguzzi and Calella, 2009; Prusiner, 1982). In yeast, self-propagation of prion translation termination factor Sup35, results in a read-through of nonsense codons, allowing cells to acquire new phenotypes (Tyedmers et al., 2008). Resulting genetic variation proved advantageous for cells, especially under stress conditions (Tyedmers et al., 2008). In recent years, protein aggregation was recognized as an adaptive cellular response (Saarikangas and Barral, 2016). For example, ATP-dependent widespread aggregation regulates cellular dormancy in bacteria ( $\mathrm{Pu}$ et al., 2019), formation of insoluble inclusions in yeast protects cells from oxidative stress (Carija et al., 2017), recruitment of misfolded proteins to inclusions regulates replicative rejuvenation in yeast an mammalian cells (Ogrodnik et al., 2014; Saarikangas and Barral, 2015), aggregation of metabolic enzymes in yeast during stress preserves proteins from degradation aiding adaptation (Petrovska et al., 2014).

Studying mechanisms of inclusion formation revealed functional modes of inclusions, that were surprisingly similar between distinct non membrane compartments arising from the basic properties of protein aggregation (Kaganovich, 2017). Clustering of functional proteins inside the inclusions allows (1) amplification of protein function. For example, the factory of enzymes that synthesize purines in eukaryotic cells has been found to assemble into an aggregate, referred to as Purinosome, that carried out the biosynthesis of purines (An et al., 2008; Pedley and Benkovic, 2017). Not only the proteins are functional inside the Purinosome, inducing its formation stimulates the production of purines (Zhao et al., 2015). Another side of protein clustering is their (2) storage. Preservation of proteins inside the inclusions protects them from degradation and also 
serves as a readily available protein pool for future use (Marshall and Vierstra, 2018; Petrovska et al., 2014; Sagot et al., 2006). Upon starvation yeast assemble proteasome subunits in the cytoplasmic inclusion, the IPOD, protecting them from autophagic degradation (Marshall and Vierstra, 2018). Another side of protein storage in the inclusion is temporal inactivation of proteins. Aggregation can change the stoichiometry of protein complexes, regulating diverse cellular functions from transcription to dosage compensation of protein copy numbers in aneuploidy (Brennan et al., 2019; Oeser et al., 2016). Cells can exert control over the phase separated inclusion, (3) filtering proteins by tuning the concentration and availability of the scaffold proteins (Banani et al., 2016). This function comes to play in protein quality control inclusions. Chaperones selectively bind damaged proteins, essentially filtering the cytoplasm and recruiting them to inclusions (Kaganovich et al., 2008; McClellan et al., 2005b; Spokoini et al., 2012). This results in distinct protein quality control compartments that play central roles in protein homeostasis and replicative rejuvenation (Gidalevitz et al., 2011; Ogrodnik et al., 2014). Finally, reciprocal regulation of signaling by many RNP granules allows aggregates to (4) sense cellular state and partake in life-death decisions. For example, Stress Granules recruit mTOR kinase and are thought to inhibit apoptosis (Thedieck et al., 2013). Hence, inclusion formation is a robust platform for executing diverse cellular functions (Kaganovich, 2017). 


\subsection{References}

Aditi, Mason, A.C., Sharma, M., Dawson, T.R., and Wente, S.R. (2019). MAPK- and glycogen synthase kinase 3-mediated phosphorylation regulates the DEAD-box protein modulator Gle1 for control of stress granule dynamics. J Biol Chem 294, 559-575.

Aguzzi, A., and Calella, A.M. (2009). Prions: protein aggregation and infectious diseases. Physiol Rev 89, $1105-1152$.

Alberti, S. (2012). Molecular mechanisms of spatial protein quality control. Prion 6, 437-442.

Amen, T., and Kaganovich, D. (2015). Dynamic droplets: the role of cytoplasmic inclusions in stress, function, and disease. Cell Mol Life Sci 72, 401-415.

Amen, T., Lázaro, D.F., Outeiro, T.F., and Kaganovich, D. (2015). Modeling Neuronal Pathology in Yeast: Insights into the Molecular Basis of Parkinson's Disease. Israel Journal of Chemistry 55, 1252-1259.

An, S., Kumar, R., Sheets, E.D., and Benkovic, S.J. (2008). Reversible compartmentalization of de novo purine biosynthetic complexes in living cells. Science 320, 103-106.

Anderson, P., and Kedersha, N. (2002). Visibly stressed: the role of eIF2, TIA-1, and stress granules in protein translation. Cell Stress Chaperones 7, 213-221.

Andorfer, C., Kress, Y., Espinoza, M., de Silva, R., Tucker, K.L., Barde, Y.A., Duff, K., and Davies, P. (2003). Hyperphosphorylation and aggregation of tau in mice expressing normal human tau isoforms. J Neurochem 86, 582-590.

Arimoto, K., Fukuda, H., Imajoh-Ohmi, S., Saito, H., and Takekawa, M. (2008). Formation of stress granules inhibits apoptosis by suppressing stress-responsive MAPK pathways. Nat Cell Biol 10, 13241332.

Aulas, A., Fay, M.M., Lyons, S.M., Achorn, C.A., Kedersha, N., Anderson, P., and Ivanov, P. (2017). Stress-specific differences in assembly and composition of stress granules and related foci. J Cell Sci 130, 927-937.

Banani, S.F., Rice, A.M., Peeples, W.B., Lin, Y., Jain, S., Parker, R., and Rosen, M.K. (2016). Compositional Control of Phase-Separated Cellular Bodies. Cell 166, 651-663.

Bashkirov, V.I., Scherthan, H., Solinger, J.A., Buerstedde, J.M., and Heyer, W.D. (1997). A mouse cytoplasmic exoribonuclease (mXRN1p) with preference for G4 tetraplex substrates. J Cell Biol 136, 761773.

Bence, N.F., Sampat, R.M., and Kopito, R.R. (2001). Impairment of the ubiquitin-proteasome system by protein aggregation. Science 292, 1552-1555.

Boeynaems, S., Alberti, S., Fawzi, N.L., Mittag, T., Polymenidou, M., Rousseau, F., Schymkowitz, J., Shorter, J., Wolozin, B., Van Den Bosch, L., et al. (2018). Protein Phase Separation: A New Phase in Cell Biology. Trends Cell Biol 28, 420-435.

Brangwynne, C.P., Eckmann, C.R., Courson, D.S., Rybarska, A., Hoege, C., Gharakhani, J., Julicher, F., and Hyman, A.A. (2009). Germline P granules are liquid droplets that localize by controlled dissolution/condensation. Science 324, 1729-1732.

Brennan, C.M., Vaites, L.P., Wells, J.N., Santaguida, S., Paulo, J.A., Storchova, Z., Harper, J.W., Marsh, J.A., and Amon, A. (2019). Protein aggregation mediates stoichiometry of protein complexes in aneuploid cells. Genes Dev 33, 1031-1047.

Brock, K.P., Abraham, A.C., Amen, T., Kaganovich, D., and England, J.L. (2015). Structural Basis for Modulation of Quality Control Fate in a Marginally Stable Protein. Structure 23, 1169-1178.

Bruderek, M., Jaworek, W., Wilkening, A., Rub, C., Cenini, G., Fortsch, A., Sylvester, M., and Voos, W. (2018). IMiQ: a novel protein quality control compartment protecting mitochondrial functional integrity. Mol Biol Cell 29, 256-269.

Buchan, J.R., Kolaitis, R.M., Taylor, J.P., and Parker, R. (2013). Eukaryotic stress granules are cleared by autophagy and Cdc48/VCP function. Cell 153, 1461-1474.

Buchan, J.R., and Parker, R. (2009). Eukaryotic Stress Granules: The Ins and Outs of Translation. Molecular Cell 36, 932-941. 
Buchan, J.R., Yoon, J.H., and Parker, R. (2011). Stress-specific composition, assembly and kinetics of stress granules in Saccharomyces cerevisiae. J Cell Sci 124, 228-239.

Carija, A., Navarro, S., de Groot, N.S., and Ventura, S. (2017). Protein aggregation into insoluble deposits protects from oxidative stress. Redox Biol 12, 699-711.

Clemens, M.J. (2001). Initiation factor eIF2 alpha phosphorylation in stress responses and apoptosis. Prog Mol Subcell Biol 27, 57-89.

David, D.C., Ollikainen, N., Trinidad, J.C., Cary, M.P., Burlingame, A.L., and Kenyon, C. (2010). Widespread protein aggregation as an inherent part of aging in C. elegans. PLoS Biol 8, e1000450.

De Graeve, F., and Besse, F. (2018). Neuronal RNP granules: from physiological to pathological assemblies. Biol Chem 399, 623-635.

Deiana, A., Forcelloni, S., Porrello, A., and Giansanti, A. (2019). Intrinsically disordered proteins and structured proteins with intrinsically disordered regions have different functional roles in the cell. PLoS One 14, e0217889.

Dewey, C.M., Cenik, B., Sephton, C.F., Johnson, B.A., Herz, J., and Yu, G. (2012). TDP-43 aggregation in neurodegeneration: Are stress granules the key? Brain Research 1462, 16-25.

Du, K., Karp, P.H., Ackerley, C., Zabner, J., Keshavjee, S., Cutz, E., and Yeger, H. (2015). Aggregates of mutant CFTR fragments in airway epithelial cells of CF lungs: new pathologic observations. J Cyst Fibros $14,182-193$.

Dunker, A.K., Cortese, M.S., Romero, P., Iakoucheva, L.M., and Uversky, V.N. (2005). Flexible nets. The roles of intrinsic disorder in protein interaction networks. FEBS J 272, 5129-5148.

Dyson, H.J., and Wright, P.E. (2005). Intrinsically unstructured proteins and their functions. Nat Rev Mol Cell Biol 6, 197-208.

Elbaum-Garfinkle, S., Kim, Y., Szczepaniak, K., Chen, C.C., Eckmann, C.R., Myong, S., and Brangwynne, C.P. (2015). The disordered P granule protein LAF-1 drives phase separation into droplets with tunable viscosity and dynamics. Proc Natl Acad Sci U S A 112, 7189-7194.

Ellis, R.J. (2001). Macromolecular crowding: obvious but underappreciated. Trends Biochem Sci 26, 597604.

Eystathioy, T., Chan, E.K., Tenenbaum, S.A., Keene, J.D., Griffith, K., and Fritzler, M.J. (2002). A phosphorylated cytoplasmic autoantigen, GW182, associates with a unique population of human mRNAs within novel cytoplasmic speckles. Mol Biol Cell 13, 1338-1351.

Feric, M., Vaidya, N., Harmon, T.S., Mitrea, D.M., Zhu, L., Richardson, T.M., Kriwacki, R.W., Pappu, R.V., and Brangwynne, C.P. (2016). Coexisting Liquid Phases Underlie Nucleolar Subcompartments. Cell 165, 1686-1697.

Fingar, D.C., Richardson, C.J., Tee, A.R., Cheatham, L., Tsou, C., and Blenis, J. (2004). mTOR controls cell cycle progression through its cell growth effectors S6K1 and 4E-BP1/eukaryotic translation initiation factor 4E. Mol Cell Biol 24, 200-216.

Fink, A.L. (1998). Protein aggregation: folding aggregates, inclusion bodies and amyloid. Fold Des 3, R923.

Forman-Kay, J.D., and Mittag, T. (2013). From sequence and forces to structure, function, and evolution of intrinsically disordered proteins. Structure 21, 1492-1499.

Fournier, M.J., Coudert, L., Mellaoui, S., Adjibade, P., Gareau, C., Cote, M.F., Sonenberg, N., Gaudreault, R.C., and Mazroui, R. (2013). Inactivation of the mTORC1-eukaryotic translation initiation factor 4E pathway alters stress granule formation. Mol Cell Biol 33, 2285-2301.

Franzmann, T.M., and Alberti, S. (2019). Prion-like low-complexity sequences: Key regulators of protein solubility and phase behavior. J Biol Chem 294, 7128-7136.

Gidalevitz, T., Prahlad, V., and Morimoto, R.I. (2011). The stress of protein misfolding: from single cells to multicellular organisms. Cold Spring Harb Perspect Biol 3.

Gilbertson, S., Federspiel, J.D., Hartenian, E., Cristea, I.M., and Glaunsinger, B. (2018). Changes in mRNA abundance drive shuttling of RNA binding proteins, linking cytoplasmic RNA degradation to transcription. Elife 7. 
Gilks, N., Kedersha, N., Ayodele, M., Shen, L., Stoecklin, G., Dember, L.M., and Anderson, P. (2004). Stress granule assembly is mediated by prion-like aggregation of TIA-1. Mol Biol Cell 15, 5383-5398. Gingras, A.C., Gygi, S.P., Raught, B., Polakiewicz, R.D., Abraham, R.T., Hoekstra, M.F., Aebersold, R., and Sonenberg, N. (1999). Regulation of 4E-BP1 phosphorylation: a novel two-step mechanism. Genes \& Development 13, 1422-1437.

Goldberg, A.L. (2003). Protein degradation and protection against misfolded or damaged proteins. Nature 426, 895-899.

Gomes, E., and Shorter, J. (2019). The molecular language of membraneless organelles. J Biol Chem 294, 7115-7127.

Gorbenko, G., and Trusova, V. (2011). Protein aggregation in a membrane environment. Adv Protein Chem Struct Biol 84, 113-142.

Gundersen, V. (2010). Protein aggregation in Parkinson's disease. Acta Neurol Scand Suppl, 82-87.

Guo, L., Kim, H.J., Wang, H., Monaghan, J., Freyermuth, F., Sung, J.C., O'Donovan, K., Fare, C.M., Diaz, Z., Singh, N., et al. (2018). Nuclear-Import Receptors Reverse Aberrant Phase Transitions of RNA-Binding Proteins with Prion-like Domains. Cell 173, 677-692 e620.

Hayes, M.H., Peuchen, E.H., Dovichi, N.J., and Weeks, D.L. (2018). Dual roles for ATP in the regulation of phase separated protein aggregates in Xenopus oocyte nucleoli. Elife 7.

Heberle, A.M., Razquin Navas, P., Langelaar-Makkinje, M., Kasack, K., Sadik, A., Faessler, E., Hahn, U., Marx-Stoelting, P., Opitz, C.A., Sers, C., et al. (2019). The PI3K and MAPK/p38 pathways control stress granule assembly in a hierarchical manner. Life Sci Alliance 2.

Henry, C.J. (2000). Mechanisms of changes in basal metabolism during ageing. Eur J Clin Nutr 54 Suppl 3, S77-91.

Hershko, A., and Ciechanover, A. (1998). The ubiquitin system. Annu Rev Biochem 67, 425-479.

Hill, S.M., Hao, X., Gronvall, J., Spikings-Nordby, S., Widlund, P.O., Amen, T., Jorhov, A., Josefson, R., Kaganovich, D., Liu, B., et al. (2016). Asymmetric Inheritance of Aggregated Proteins and Age Reset in Yeast Are Regulated by Vac17-Dependent Vacuolar Functions. Cell Rep 16, 826-838.

Ingelfinger, D., Arndt-Jovin, D.J., Luhrmann, R., and Achsel, T. (2002). The human LSm1-7 proteins colocalize with the mRNA-degrading enzymes Dcp $1 / 2$ and Xrnl in distinct cytoplasmic foci. RNA 8,1489 1501.

Irvine, G.B., El-Agnaf, O.M., Shankar, G.M., and Walsh, D.M. (2008). Protein aggregation in the brain: the molecular basis for Alzheimer's and Parkinson's diseases. Mol Med 14, 451-464.

Jevtov, I., Zacharogianni, M., van Oorschot, M.M., van Zadelhoff, G., Aguilera-Gomez, A., Vuillez, I., Braakman, I., Hafen, E., Stocker, H., and Rabouille, C. (2015). TORC2 mediates the heat stress response in Drosophila by promoting the formation of stress granules. J Cell Sci 128, 2497-2508.

Johnston, J.A., Ward, C.L., and Kopito, R.R. (1998). Aggresomes: a cellular response to misfolded proteins. J Cell Biol 143, 1883-1898.

Jones, R.D., and Gardner, R.G. (2015). Digging for Buried Amino Acids Unearths New Protein Quality Control Treasure. Structure 23, 1151-1152.

Jouanne, M., Rault, S., and Voisin-Chiret, A.S. (2017). Tau protein aggregation in Alzheimer's disease: An attractive target for the development of novel therapeutic agents. Eur J Med Chem 139, 153-167.

Kaganovich, D. (2017). There Is an Inclusion for That: Material Properties of Protein Granules Provide a Platform for Building Diverse Cellular Functions. Trends Biochem Sci 42, 765-776.

Kaganovich, D., Kopito, R., and Frydman, J. (2008). Misfolded proteins partition between two distinct quality control compartments. Nature 454, 1088-U1036.

Kamura, T., Brower, C.S., Conaway, R.C., and Conaway, J.W. (2002). A molecular basis for stabilization of the von Hippel-Lindau (VHL) tumor suppressor protein by components of the VHL ubiquitin ligase. J Biol Chem 277, 30388-30393.

Kato, M., Han, T.W., Xie, S., Shi, K., Du, X., Wu, L.C., Mirzaei, H., Goldsmith, E.J., Longgood, J., Pei, J., et al. (2012). Cell-free formation of RNA granules: low complexity sequence domains form dynamic fibers within hydrogels. Cell 149, 753-767. 
Kaur, T., Alshareedah, I., Wang, W., Ngo, J., Moosa, M.M., and Banerjee, P.R. (2019). Molecular Crowding Tunes Material States of Ribonucleoprotein Condensates. Biomolecules 9.

Kedersha, N., and Anderson, P. (2002). Stress granules: sites of mRNA triage that regulate mRNA stability and translatability. Biochem Soc Trans 30, 963-969.

Kedersha, N., and Anderson, P. (2007). Mammalian stress granules and processing bodies. Methods Enzymol 431, 61-81.

Kedersha, N., Cho, M.R., Li, W., Yacono, P.W., Chen, S., Gilks, N., Golan, D.E., and Anderson, P. (2000). Dynamic shuttling of TIA-1 accompanies the recruitment of mRNA to mammalian stress granules. J Cell Biol 151, 1257-1268.

Kedersha, N., Ivanov, P., and Anderson, P. (2013). Stress granules and cell signaling: more than just a passing phase? Trends Biochem Sci 38, 494-506.

Kedersha, N., Panas, M.D., Achorn, C.A., Lyons, S., Tisdale, S., Hickman, T., Thomas, M., Lieberman, J., McInerney, G.M., Ivanov, P., et al. (2016a). G3BP-Caprin1-USP10 complexes mediate stress granule condensation and associate with 40S subunits. J Cell Biol 212, 845-860.

Kedersha, N., Panas, M.D., Achorn, C.A., Lyons, S., Tisdale, S., Hickman, T., Thomas, M., Lieberman, J., McInerney, G.M., Ivanov, P., et al. (2016b). G3BP-Caprin1-USP10 complexes mediate stress granule condensation and associate with 40S subunits. The Journal of Cell Biology 212, 845-860.

Kedersha, N., Stoecklin, G., Ayodele, M., Yacono, P., Lykke-Andersen, J., Fritzler, M.J., Scheuner, D., Kaufman, R.J., Golan, D.E., and Anderson, P. (2005). Stress granules and processing bodies are dynamically linked sites of mRNP remodeling. J Cell Biol 169, 871-884.

Kobayashi, T., Winslow, S., Sunesson, L., Hellman, U., and Larsson, C. (2012). PKCalpha binds G3BP2 and regulates stress granule formation following cellular stress. PLoS One 7, e35820.

Krumova, P., Meulmeester, E., Garrido, M., Tirard, M., Hsiao, H.H., Bossis, G., Urlaub, H., Zweckstetter, M., Kugler, S., Melchior, F., et al. (2011). Sumoylation inhibits alpha-synuclein aggregation and toxicity. J Cell Biol 194, 49-60.

Kuo, D., Nie, M., and Courey, A.J. (2014). SUMO as a solubility tag and in vivo cleavage of SUMO fusion proteins with Ulp1. Methods Mol Biol 1177, 71-80.

Lamark, T., and Johansen, T. (2012). Aggrephagy: selective disposal of protein aggregates by macroautophagy. Int J Cell Biol 2012, 736905.

Lecker, S.H., Goldberg, A.L., and Mitch, W.E. (2006). Protein degradation by the ubiquitin-proteasome pathway in normal and disease states. J Am Soc Nephrol 17, 1807-1819.

Lee, J.E., Cathey, P.I., Wu, H., Parker, R., and Voeltz, G.K. (2020). Endoplasmic reticulum contact sites regulate the dynamics of membraneless organelles. Science 367.

Lelouard, H., Ferrand, V., Marguet, D., Bania, J., Camosseto, V., David, A., Gatti, E., and Pierre, P. (2004). Dendritic cell aggresome-like induced structures are dedicated areas for ubiquitination and storage of newly synthesized defective proteins. J Cell Biol 164, 667-675.

Li, Y.R., King, O.D., Shorter, J., and Gitler, A.D. (2013). Stress granules as crucibles of ALS pathogenesis. J Cell Biol 201, 361-372.

Marshall, R.S., and Vierstra, R.D. (2018). Proteasome storage granules protect proteasomes from autophagic degradation upon carbon starvation. Elife 7.

Mateju, D., Franzmann, T.M., Patel, A., Kopach, A., Boczek, E.E., Maharana, S., Lee, H.O., Carra, S., Hyman, A.A., and Alberti, S. (2017). An aberrant phase transition of stress granules triggered by misfolded protein and prevented by chaperone function. EMBO J 36, 1669-1687.

McClellan, A.J., Scott, M.D., and Frydman, J. (2005a). Folding and quality control of the VHL tumor suppressor proceed through distinct chaperone pathways. Cell 121, 739-748.

McClellan, A.J., Tam, S., Kaganovich, D., and Frydman, J. (2005b). Protein quality control: chaperones culling corrupt conformations. Nat Cell Biol 7, 736-741.

McInerney, G.M., Kedersha, N.L., Kaufman, R.J., Anderson, P., and Liljestrom, P. (2005). Importance of eIF2alpha phosphorylation and stress granule assembly in alphavirus translation regulation. Mol Biol Cell $16,3753-3763$. 
Mogk, A., and Bukau, B. (2014). Mitochondria tether protein trash to rejuvenate cellular environments. Cell 159, 471-472.

Moldavski, O., Amen, T., Levin-Zaidman, S., Eisenstein, M., Rogachev, I., Brandis, A., Kaganovich, D., and Schuldiner, M. (2015). Lipid Droplets Are Essential for Efficient Clearance of Cytosolic Inclusion Bodies. Dev Cell 33, 603-610.

Oeser, M.L., Amen, T., Nadel, C.M., Bradley, A.I., Reed, B.J., Jones, R.D., Gopalan, J., Kaganovich, D., and Gardner, R.G. (2016). Dynamic Sumoylation of a Conserved Transcription Corepressor Prevents Persistent Inclusion Formation during Hyperosmotic Stress. PLoS Genet 12, e1005809.

Ogrodnik, M., Salmonowicz, H., Brown, R., Turkowska, J., Sredniawa, W., Pattabiraman, S., Amen, T., Abraham, A.C., Eichler, N., Lyakhovetsky, R., et al. (2014). Dynamic JUNQ inclusion bodies are asymmetrically inherited in mammalian cell lines through the asymmetric partitioning of vimentin. Proc Natl Acad Sci U S A 111, 8049-8054.

Oldfield, C.J., and Dunker, A.K. (2014). Intrinsically disordered proteins and intrinsically disordered protein regions. Annu Rev Biochem 83, 553-584.

Outeiro, T.F., and Lindquist, S. (2003). Yeast cells provide insight into alpha-synuclein biology and pathobiology. Science 302, 1772-1775.

Panas, M.D., Kedersha, N., Schulte, T., Branca, R.M., Ivanov, P., and Anderson, P. (2019). Phosphorylation of G3BP1-S149 does not influence stress granule assembly. J Cell Biol 218, 2425-2432.

Park, S., Lee, J.H., Jeon, J.H., and Lee, M.J. (2018). Degradation or aggregation: the ramifications of posttranslational modifications on tau. BMB Rep 51, 265-273.

Patel, A., Lee, H.O., Jawerth, L., Maharana, S., Jahnel, M., Hein, M.Y., Stoynov, S., Mahamid, J., Saha, S., Franzmann, T.M., et al. (2015). A Liquid-to-Solid Phase Transition of the ALS Protein FUS Accelerated by Disease Mutation. Cell 162, 1066-1077.

Patel, A., Malinovska, L., Saha, S., Wang, J., Alberti, S., Krishnan, Y., and Hyman, A.A. (2017). ATP as a biological hydrotrope. Science 356, 753-756.

Pedley, A.M., and Benkovic, S.J. (2017). A New View into the Regulation of Purine Metabolism: The Purinosome. Trends Biochem Sci 42, 141-154.

Peth, A., Nathan, J.A., and Goldberg, A.L. (2013). The ATP costs and time required to degrade ubiquitinated proteins by the $26 \mathrm{~S}$ proteasome. J Biol Chem 288, 29215-29222.

Petroi, D., Popova, B., Taheri-Talesh, N., Irniger, S., Shahpasandzadeh, H., Zweckstetter, M., Outeiro, T.F., and Braus, G.H. (2012). Aggregate clearance of alpha-synuclein in Saccharomyces cerevisiae depends more on autophagosome and vacuole function than on the proteasome. J Biol Chem 287, 27567-27579.

Petrovska, I., Nuske, E., Munder, M.C., Kulasegaran, G., Malinovska, L., Kroschwald, S., Richter, D., Fahmy, K., Gibson, K., Verbavatz, J.M., et al. (2014). Filament formation by metabolic enzymes is a specific adaptation to an advanced state of cellular starvation. Elife.

Protter, D.S.W., and Parker, R. (2016). Principles and Properties of Stress Granules. Trends Cell Biol 26, 668-679.

Prusiner, S.B. (1982). Novel proteinaceous infectious particles cause scrapie. Science 216, 136-144.

Pu, Y., Li, Y., Jin, X., Tian, T., Ma, Q., Zhao, Z., Lin, S.Y., Chen, Z., Li, B., Yao, G., et al. (2019). ATPDependent Dynamic Protein Aggregation Regulates Bacterial Dormancy Depth Critical for Antibiotic Tolerance. Mol Cell 73, 143-156 e144.

Reineke, L.C., Tsai, W.C., Jain, A., Kaelber, J.T., Jung, S.Y., and Lloyd, R.E. (2017). Casein Kinase 2 Is Linked to Stress Granule Dynamics through Phosphorylation of the Stress Granule Nucleating Protein G3BP1. Mol Cell Biol 37.

Saad, S., Cereghetti, G., Feng, Y., Picotti, P., Peter, M., and Dechant, R. (2017). Reversible protein aggregation is a protective mechanism to ensure cell cycle restart after stress. Nat Cell Biol 19, 1202-1213. Saarikangas, J., and Barral, Y. (2015). Protein aggregates are associated with replicative aging without compromising protein quality control. Elife 4.

Saarikangas, J., and Barral, Y. (2016). Protein aggregation as a mechanism of adaptive cellular responses. Curr Genet 62, 711-724. 
Sagot, I., Pinson, B., Salin, B., and Daignan-Fornier, B. (2006). Actin bodies in yeast quiescent cells: an immediately available actin reserve? Mol Biol Cell 17, 4645-4655.

Sancenon, V., Lee, S.A., Patrick, C., Griffith, J., Paulino, A., Outeiro, T.F., Reggiori, F., Masliah, E., and Muchowski, P.J. (2012). Suppression of alpha-synuclein toxicity and vesicle trafficking defects by phosphorylation at S129 in yeast depends on genetic context. Hum Mol Genet 21, 2432-2449.

Sasahara, K., Yamaguchi, K., So, M., and Goto, Y. (2019). Polyphosphates diminish solubility of a globular protein and thereby promote amyloid aggregation. J Biol Chem 294, 15318-15329.

Schoenfeld, A.R., Davidowitz, E.J., and Burk, R.D. (2000). Elongin BC complex prevents degradation of von Hippel-Lindau tumor suppressor gene products. Proc Natl Acad Sci U S A 97, 8507-8512.

Schuster, B.S., Reed, E.H., Parthasarathy, R., Jahnke, C.N., Caldwell, R.M., Bermudez, J.G., Ramage, H., Good, M.C., and Hammer, D.A. (2018). Controllable protein phase separation and modular recruitment to form responsive membraneless organelles. Nat Commun 9, 2985.

Sfakianos, A.P., Mellor, L.E., Pang, Y.F., Kritsiligkou, P., Needs, H., Abou-Hamdan, H., Desaubry, L., Poulin, G.B., Ashe, M.P., and Whitmarsh, A.J. (2018). The mTOR-S6 kinase pathway promotes stress granule assembly. Cell Death Differ 25, 1766-1780.

Shiber, A., Breuer, W., Brandeis, M., and Ravid, T. (2013). Ubiquitin conjugation triggers misfolded protein sequestration into quality control foci when Hsp70 chaperone levels are limiting. Mol Biol Cell 24, 2076-2087.

Shin, Y., and Brangwynne, C.P. (2017). Liquid phase condensation in cell physiology and disease. Science 357.

Shorter, J. (2011). The mammalian disaggregase machinery: Hsp110 synergizes with Hsp70 and Hsp40 to catalyze protein disaggregation and reactivation in a cell-free system. PLoS One 6, e26319.

Shorter, J., and Southworth, D.R. (2019). Spiraling in Control: Structures and Mechanisms of the Hsp104 Disaggregase. Cold Spring Harb Perspect Biol 11.

Si, K., and Kandel, E.R. (2016). The Role of Functional Prion-Like Proteins in the Persistence of Memory. Cold Spring Harb Perspect Biol 8, a021774.

Soper, J.H., Roy, S., Stieber, A., Lee, E., Wilson, R.B., Trojanowski, J.Q., Burd, C.G., and Lee, V.M. (2008). Alpha-synuclein-induced aggregation of cytoplasmic vesicles in Saccharomyces cerevisiae. Mol Biol Cell 19, 1093-1103.

Spokoini, R., Moldavski, O., Nahmias, Y., England, J.L., Schuldiner, M., and Kaganovich, D. (2012). Confinement to organelle-associated inclusion structures mediates asymmetric inheritance of aggregated protein in budding yeast. Cell Rep 2, 738-747.

Szeto, J., Kaniuk, N.A., Canadien, V., Nisman, R., Mizushima, N., Yoshimori, T., Bazett-Jones, D.P., and Brumell, J.H. (2006). ALIS are stress-induced protein storage compartments for substrates of the proteasome and autophagy. Autophagy 2, 189-199.

Takahashi, M., Higuchi, M., Matsuki, H., Yoshita, M., Ohsawa, T., Oie, M., and Fujii, M. (2013). Stress granules inhibit apoptosis by reducing reactive oxygen species production. Mol Cell Biol 33, 815-829.

Tenreiro, S., Reimao-Pinto, M.M., Antas, P., Rino, J., Wawrzycka, D., Macedo, D., Rosado-Ramos, R., Amen, T., Waiss, M., Magalhaes, F., et al. (2014). Phosphorylation modulates clearance of alpha-synuclein inclusions in a yeast model of Parkinson's disease. PLoS Genet 10, e1004302.

Thedieck, K., Holzwarth, B., Prentzell, M.T., Boehlke, C., Klasener, K., Ruf, S., Sonntag, A.G., Maerz, L., Grellscheid, S.N., Kremmer, E., et al. (2013). Inhibition of mTORC1 by astrin and stress granules prevents apoptosis in cancer cells. Cell 154, 859-874.

Tyedmers, J., Madariaga, M.L., and Lindquist, S. (2008). Prion switching in response to environmental stress. PLoS Biol 6, e294.

Tzamarias, D., and Struhl, K. (1994). Functional dissection of the yeast Cyc8-Tup1 transcriptional corepressor complex. Nature 369, 758-761.

Ulmer, T.S., Bax, A., Cole, N.B., and Nussbaum, R.L. (2005). Structure and dynamics of micelle-bound human alpha-synuclein. J Biol Chem 280, 9595-9603. 
van Dijk, E., Cougot, N., Meyer, S., Babajko, S., Wahle, E., and Seraphin, B. (2002). Human Dcp2: a catalytically active mRNA decapping enzyme located in specific cytoplasmic structures. EMBO J 21, 69156924.

Vaquer-Alicea, J., and Diamond, M.I. (2019). Propagation of Protein Aggregation in Neurodegenerative Diseases. Annu Rev Biochem 88, 785-810.

Wang, J.T., and Seydoux, G. (2014). P granules. Curr Biol 24, R637-R638.

Weber, S.C., and Brangwynne, C.P. (2012). Getting RNA and protein in phase. Cell 149, 1188-1191.

Weids, A.J., Ibstedt, S., Tamas, M.J., and Grant, C.M. (2016). Distinct stress conditions result in aggregation of proteins with similar properties. Sci Rep 6, 24554.

Weisberg, S.J., Lyakhovetsky, R., Werdiger, A.C., Gitler, A.D., Soen, Y., and Kaganovich, D. (2012). Compartmentalization of superoxide dismutase 1 (SOD1G93A) aggregates determines their toxicity. Proc Natl Acad Sci U S A 109, 15811-15816.

Wolozin, B., and Ivanov, P. (2019). Stress granules and neurodegeneration. Nat Rev Neurosci 20, 649-666. Wootton, J.C. (1994). Non-globular domains in protein sequences: automated segmentation using complexity measures. Comput Chem 18, 269-285.

Wright, P.E., and Dyson, H.J. (2015). Intrinsically disordered proteins in cellular signalling and regulation. Nat Rev Mol Cell Biol 16, 18-29.

Wu, C.C., Hou, S., Orr, B.A., Kuo, B.R., Youn, Y.H., Ong, T., Roth, F., Eberhart, C.G., Robinson, G.W., Solecki, D.J., et al. (2017). mTORC1-Mediated Inhibition of 4EBP1 Is Essential for Hedgehog SignalingDriven Translation and Medulloblastoma. Dev Cell 43, 673-688 e675.

Yang, X., Hu, Z., Zhang, Q., Fan, S., Zhong, Y., Guo, D., Qin, Y., and Chen, M. (2019). SG formation relies on eIF4GI-G3BP interaction which is targeted by picornavirus stress antagonists. Cell Discov $5,1$. Yewdell, J.W., Anton, L.C., and Bennink, J.R. (1996). Defective ribosomal products (DRiPs): a major source of antigenic peptides for MHC class I molecules? J Immunol 157, 1823-1826.

Zhang, C.M., Yamaguchi, K., So, M., Sasahara, K., Ito, T., Yamamoto, S., Narita, I., Kardos, J., Naiki, H., and Goto, Y. (2019). Possible mechanisms of polyphosphate-induced amyloid fibril formation of beta2microglobulin. Proc Natl Acad Sci U S A 116, 12833-12838.

Zhang, K., Daigle, J.G., Cunningham, K.M., Coyne, A.N., Ruan, K., Grima, J.C., Bowen, K.E., Wadhwa, H., Yang, P., Rigo, F., et al. (2018a). Stress Granule Assembly Disrupts Nucleocytoplasmic Transport. Cell 173, 958-971 e917.

Zhang, K., Daigle, J.G., Cunningham, K.M., Coyne, A.N., Ruan, K., Grima, J.C., Bowen, K.E., Wadhwa, H., Yang, P., Rigo, F., et al. (2018b). Stress Granule Assembly Disrupts Nucleocytoplasmic Transport. Cell 173, 958-971.e917.

Zhao, H., Chiaro, C.R., Zhang, L., Smith, P.B., Chan, C.Y., Pedley, A.M., Pugh, R.J., French, J.B., Patterson, A.D., and Benkovic, S.J. (2015). Quantitative analysis of purine nucleotides indicates that purinosomes increase de novo purine biosynthesis. J Biol Chem 290, 6705-6713. 


\section{Chapter 2}

\section{Stress Granule regulation of fatty acid oxidation}

If we are to study the function of granules in the cellular context, we ought to consider their formation in physiological stress conditions. Stress Granules appear in heat stress, during longterm or chronic glucose deprivation, as well as oxidative stress, which makes them a general stress

response pathway. In the following chapter we discuss Stress Granule functionality in a physiological setting of a long-term nutrient starvation primarily using mammalian cells. 


\section{Summary}

The formation of Stress Granules (SG) is an essential aspect of the cellular response to several kinds of stress, but its adaptive role is as yet far from being completely clear. SG dysfunction is implicated in aging-onset neurodegenerative diseases (NDs), prompting interest in the physiological function of SGs. Here, we report that during chronic starvation stress SGs interact with mitochondria and Lipid Droplets and regulate metabolic remodeling. We show that SG formation leads to a down-regulation of Fatty Acid Oxidation (FAO) through a signaling cascade that involves GSK3 kinase activation. SGs interact with a mitochondrial porin, VDAC2, triggering its phosphorylation. Phosphorylated VDAC2 is then depleted from the mitochondrial membrane and forms reversible inactive clusters, thereby blocking fatty acid (FA) import into mitochondria and promoting Lipid Droplet (LD) biogenesis. The subsequent decrease in FAO during long-term starvation reduces oxidative damage and rations fatty acids for longer potential use. We propose a unifying model of SGs as master-regulators of the metabolic response to starvation, responsible for controlling Lipid Droplet association with mitochondria, managing kinase signaling, and regulating lipid flux. Since metabolic dysfunction is a common pathological element of NDs, our findings provide a new direction for studying the clinical relevance of SGs.

\section{Introduction}

Stress Granule formation is a common feature of otherwise unrelated stress phenomena, which include heat stress, oxidative stress (e.g. arsenite), UV irradiation, ER stress, viral infection, and chronic nutrient starvation (Buchan et al., 2011; Jevtov et al., 2015; Moutaoufik et al., 2014; Piotrowska et al., 2010; Reineke et al., 2018; Walker et al., 2013). In certain cases, the protective function of SGs has been described. For example, during acute stress SGs inhibit pro-apoptotic kinases and therefore promote survival (Arimoto et al., 2008). In other instances, the connection between SGs and stress-resistance is less clear. A number of additional functions have been reported for SGs, including mRNA quality control, spatiotemporal control of gene expression, regulation of nucleocytoplasmic transport, preventing pathological protein aggregation, and alleviating ROS damage (Kedersha and Anderson, 2007; Kedersha et al., 2013; Kedersha et al., 2005; Khong et al., 2017; Mann et al., 2019; McGurk et al., 2018; Panas et al., 2016; Takahashi et 
al., 2013; Zhang et al., 2018a; Zhang et al., 2018b). In the latter case, SGs are shown to be activated by oxidative stress (Kato et al., 2019), and to promote survival by activating the antioxidant activity of USP10 thereby reducing Reactive Oxygen Species (ROS) production (Takahashi et al., 2013). In several other stress conditions, however, the precise role of SGs not known. Additionally, it is not clear whether SGs carry out specific functions at different times and stresses, or whether their versatility allows them to simultaneously exert control over multiple levels of stress response by driving multiple functions all at once. Overall, the importance of SGs to cell survival is best demonstrated by disease states that are apparently brought about by SG dysfunction (Aulas and Vande Velde, 2015; Dewey et al., 2012; Li et al., 2013a). Many instances of a heritable form of Amyotrophic Lateral Sclerosis (ALS), an aging-triggered neurodegenerative disease (ND), are attributed to mutations in SG constituent proteins or RNAs (Dewey et al., 2012; Lenzi et al., 2015; Li et al., 2013a; Mackenzie et al., 2017). These include the RNA-binding proteins TDP43, FUS, superoxide dismutase SOD1, C9ORF72, and others (Taylor et al., 2016). Some of these mutations, such as those in FUS and SOD1, have been shown to alter the physical properties of SGs thus compromising their function (Mateju et al., 2017; Patel et al., 2015), and others (e.g. TDP43 Q331K mutant) titrate a critical component of SGs, thereby suppressing their formation (Gordon et al., 2019; Orru et al., 2016). The physiological functions of SGs are therefore of great interest, particularly in the context of seeking to understand the precise set of protections afforded by SGs to neurons, and the way in which their breakdown leads to pathology.

We hypothesized that metabolic stress presents an opportunity to investigate SG function in a context that is uniquely relevant to ND pathology. Long-term chronic starvation is a known physiological SG trigger (Buchan et al., 2011; Fritz et al., 2015; Reineke et al., 2018), leading to the assembly of SGs with distinct composition through a pathway that is initiated by mTORC1 inhibition, leading to eIF4E dephosphorylation and inhibition of protein synthesis (Gingras et al., 1999; Reineke et al., 2018; Sengupta et al., 2010; Sfakianos et al., 2018). Chronic stress is in line with the age-induced emergence of ND pathology which spans decades, rather than be triggered by acute stress. Moreover, the response to nutrient starvation may be of particular importance to neurons, which are thought to have unusually high energy needs in specific sub-cellular environments for operating ion channels, transporting organelles across the axon, and maintaining a functional synapse (Knobloch et al., 2017; Pennetta and Welte, 2018). Indeed, most NDs have a mitochondrial dysfunction component, leading to aberrant metabolism (Johri and Beal, 2012; 
Lahtvee et al., 2016; Vandoorne et al., 2018). Pointedly, ALS can also be viewed as a set of metabolic problems, particularly hypermetabolism, which is characterized by increased energy demand, and a shift from glucose-fueled metabolism to the hyperactive depletion of lipid energy reserves (Liu et al., 2018; Pennetta and Welte, 2018; Szelechowski et al., 2018; Tefera and Borges, 2017).

During nutrient starvation mammalian cells reconfigure their metabolic networks in order to switch from glucose metabolism towards a greater reliance on lipids (Sengupta et al., 2010). Although a highly efficient energy source, reliance on lipids is not without drawbacks. In order to make the shift to fatty acid $\beta$-oxidation (FAO), cells must deploy fatty-acids (FAs) stored as highenergy triacylglycerols in Lipid Droplets (LDs). FAs must then be imported into mitochondria for $\beta$-oxidation and for use in the citric acid cycle, requiring mitochondrial structural rearrangements. In order to be transferred to mitochondria, FAs must be liberated from LDs by lipases on the LD surface, or by lysosomal lipophagy during longer starvation bouts. However, despite the increase in lipid consumption, during starvation LD number and size typically increases (Nguyen et al., 2017). The reason for this might be that FAs in LDs need to be replenished for use in subsequent FAO, produced either through lipophagy or de novo synthesis. Severe depletion of lipids can fatally damage membranes, while high concentrations of free cytoplasmic FAs are toxic (Listenberger et al., 2003; Lu et al., 2010). In addition to the toxicity caused by free FAs, FAO also liberates high quantities of reactive oxygen species (ROS) and is therefore damaging to the cell in and of itself (Cortassa et al., 2017; Kajihara et al., 2017; Schonfeld and Wojtczak, 2008). It is essential that cells exert precise regulation over lipid flux, in order to produce ATP, have sufficient FAs for long-term starvation, and avoid toxic side-effects of FAO. It is thought that much of this critical regulation takes place on the mitochondrial membrane, in the form of local translation of mRNAs, LD biogenesis, lipid liberation from LDs, and mitochondrial import of FAs (Benador et al., 2019; Eliyahu et al., 2010).

Here we report that during long term nutrient starvation SGs interact with the FA import channel, which we identified to be the highly conserved VDAC2 porin. SGs association with the mitochondrial membrane, whether during starvation or in response to acute stress, results in the phosphorylation of VDAC2, which then forms reversible clusters. FAs are thus depleted from mitochondria and routed to LDs. The perturbation of this FAO inhibition process results in 
dysregulated lipid metabolism, aberrantly high ATP levels, and increased oxidative damage. Overall, our study provides novel insight for understanding the mechanism of metabolic remodeling in response to starvation and helps reconcile diverse SG functions.

\section{Results:}

\section{Stress Granule formation during starvation coincides with reduced fatty acid oxidation}

Starving cells shift their metabolism to FAO in order to maintain energy levels (Mihaylova et al., 2018). During short term starvation (0-6 hours) cells are able to maintain their ATP levels (Figure 1A-B) by upregulating FAO (Figure 1C). We verified that ATP production in starving cells is indeed driven by FAO (Figure 1D). ATP levels were dependent on the presence of the mitochondrial HADHA (Hydroxyacyl-CoA Dehydrogenase Trifunctional Multienzyme Complex Subunit Alpha) enzyme that catalyzes $\beta$-oxidation of fatty acids and were severely depleted in a CRISPR/Cas9 knockout of HADHA (Figure 1D, Supplementary Figure S1B-F). Not surprisingly levels of HADHA increase during short term starvation (Figure 1E, Supplementary Figure S1F).

Surprisingly, although FAO is a more efficient energy source than glycolysis, ATP levels nevertheless decline during long-term starvation, starting at 9 hours ((Figure 1A-B), corresponding to a decline in FAO (Figure 1F, K). Since tissues affected by different forms of Amyotrophic Lateral Sclerosis are known to aberrantly upregulate FAO (Liu et al., 2018; Szelechowski et al., 2018), we measured FAO and ATP/ADP ratios in a CRISPR/Cas9-modified cell line with a heterozygous Q331K mutation in a Stress Granule component TDP43 (Figure 1F-G; Supplementary Figure S2A-C, G) (Ling et al., 2010). Strikingly, even after 18 hours of starvation TDP43-Q331K mutants showed no decrease of FAO dependency and ATP/ADP ratio (Figure 1F). As a result, these cells accumulated oxidative damage at a faster rate than control cells, which was corrected by inhibiting FAO with etomoxir (Figure 1H-J, Figure S2F). Given the consequence of failing to shut down FAO during long-term starvation, we wanted to investigate the mechanism which senses starvation and inhibits FAO.

We focused on a starvation stress response which is known to sense nutrient availability and is also thought to be compromised in ALS, namely SG formation ((Lenzi et al., 2015; Li et al., 2013b), Supplementary Figure S2D-E). SGs form in a variety of stress conditions, including 
arsenite treatment, oxidative stress, heat stress, as well as long-term starvation (Emara et al., 2012; Hamada et al., 2018; Reineke et al., 2018; Tuite et al., 2013; Varga et al., 2011). We endogenously tagged PABPC1 with the Denrda2 fluorophore in HEK293T cells using CRISPR/Cas9 genome editing (Supplementary Figure S1G-J) (Ran et al., 2013). SG formation kinetics range from minutes with arsenite, to hours in fuel starvation media, and days in gradual nutrient depletion (Figure 1K-L, Supplementary Figure S1K-M). Interestingly, we observed that the ATP/ADP ratio decline observed in wild type cells becomes apparent around the time that SGs begin to form, around 9 hours of growth on starvation media (Figure 1B, 1L, Supplementary Figure S1A). This raised the question of whether low ATP levels cause SG formation, or whether, alternatively, SG formation affects ATP/ADP levels by regulating FAO. In order to rule out the possibility that SGs form as a result of declining ATP concentrations, we altered ATP/ADP ratio in cells carrying a PABPC1 copy endogenously tagged with the mCherry fluorophore (Figure 1M, Supplementary Figure S1N-P). SGs formed in cells with both high and low ATP levels, whereas completely depleting ATP by poisoning mitochondria with rotenone and CCCP was not sufficient to trigger SG formation (Figure 1M). Next, to rule out the possibility that SGs form as a consequence of FAO-induced oxidative stress during short term starvation, we measured oxidative damage (Supplementary Figure S1R). Despite high metabolic dependency on FAO, cells were able to maintain low levels of oxidative stress and high mitochondrial potential during short-term starvation (Supplementary Figure S1Q-R). Given this anti-correlation we posited a regulatory relationship between SGs and FAO (Figure 1K).

\section{Stress Granule formation promotes increased Fatty Acid import into Lipid Droplets}

We set out to explore the role of SGs in the regulation of FAO during metabolic adaptation to starvation. Our starting hypothesis was that SGs may have a role in reducing FAO during long term starvation (Supplementary Figure S3B). They may do so by regulating FA import into mitochondria or by regulating FA storage in the lipid droplets. LDs associate with mitochondria in order to transfer FAs for $\beta$-oxidation. To rule out the possibility that FAO decline during long term starvation is associated with reduced FA content we tracked FAs in SG-forming conditions, however, it was clear that the appearance of SGs correlates directly with the pronounced growth of LDs, not their dissolution (Figure 2A, C), and formation of LD clusters (Supplementary Figure 
S6F). Strikingly, pharmacological induction of SG formation in the absence of starvation, using arsenite treatment, and induction of SG formation by over-expression of SG components such as TDP43, both led to a corresponding acceleration in LD biogenesis (Figure 2B, D; Supplementary Figure S3F). Conversely, the disruption of SG formation with cycloheximide greatly impaired LDs biogenesis (Figure 2E-F, Supplementary Figure S3C-D), implying a dependence of LD proliferation on SGs. This suggests that FAO regulation is a function of all SGs, not only those formed during starvation.

Since LDs can accumulate FAs as well as cholesterol (Figure 2G), we measured import of different types of lipids into LDs in SG-forming conditions (Guo et al., 2009). FA import significantly increased in response to SG formation, while cholesterol import remained the same (Figure 2H-J, Supplementary Figure S3A). FA were imported into LDs independently of free FA availability (Supplementary Figure S3E). Since FAs stored in LDs can be transferred to mitochondria for FAO, these results raise the possibility that SGs control the balance of FA availability (Olzmann and Carvalho, 2018). We next measured triacyl glycerol (TAG) accumulation and glycerol release in short term and long-term starvation (Figure $2 \mathrm{~K}$ ). The data showed that glycerol is increasingly released during short term starvation, possibly due to FA release from LDs. The addition of FA to starving cells led to accumulation of TAG in long term starving cells but not in short term starvation.

In order to undergo $\beta$-oxidation, FAs that have been released from LDs are imported into mitochondria (Figure 2M). We therefore asked whether the FAO increase observed in TDP43 Q331K mutant cells during long-term starvation can be rescued by inhibiting FA transport across the mitochondrial membrane in these cells (Figure 2L). This was indeed the case: FAO dependency in these cells decreased dramatically, as did the RedOx stress and ATP/ADP ratios (Figure 1G-J). Given that the TDP43 Q331K mutant is severely impaired in its ability to form SGs (Supplementary Figure 2E), these data appear to suggest that SGs play a role in FA trafficking (Supplementary Figure S3G). This prompted us to explore the physical and functional interactions between SGs and metabolic machinery.

\section{Stress Granule interactome reveals mitochondrial and lipid droplet association}


First, we decided to investigate whether starvation-induced SGs associate with membranebound organelles responsible for metabolic regulation (ER, mitochondria, LDs, and peroxisomes) (Haimovich et al., 2016). This idea is not unprecedented as SGs have recently been reported to make physical contacts with the ER, lysosomes, and cytoskeletal elements (Cioni et al., 2019; Lee et al., 2020; Liao et al., 2019; Lin et al., 2016). We examined SG association with membrane bound compartments following 9 hours of fuel depletion, when SGs first appear (Figure 3A, Supplementary Figure S4A). Live cell confocal microscopy indicated that SGs indeed form contiguously with cytoplasmic membrane-bound organelles (Figure 3A). In order to rule-out bias and coincidental proximity we generated random SG images and scored the occurrence of random co-localization (Supplementary Figure S4B). We then carried out a pair-wise comparison of random samples to observed SG proximity and quantified the difference (Figure 3B). Three membrane bound organelles: LDs, mitochondria, and peroxisomes, showed strongly non-random association with SGs (Figure 3B). To confirm this association, we monitored SG, mitochondria, and LDs localization over time (Figure 3C). Our data show that SG-LD-mitochondria supercomplexes persist over the course of several minutes in live cells (Figure S5C), despite movement within the cell, reinforcing the observation of interaction between the three organelles involved in FA trafficking.

We next set to determine the SG interacting proteins on mitochondria and LDs. In order to understand how SGs regulate FA flux, we performed BioID analysis of the PABPC1 interactome in different SG conditions (Roux et al., 2012). We fused PABPC1 to promiscuous BirA(R118G) and GFP to be able to visualize SG formation while labeling interacting proteins (Figure 3D, Supplementary Figure S4C). BioID allows identification of interacting proteins as well as the proteome (Roux et al., 2012). The SG interactome was analyzed in starvation, arsenite, and 2bromopalmitate SG formation conditions (Figure 3D, manuscript in preparation). Control conditions were used to normalize data for PABPC1 interacting proteins in non-SG conditions. Biotinylation was started by addition of biotin to the media after SG formation and incubation for 6 hours (Supplementary Figure S4D), leading to enrichment of biotinylation in SGs (Supplementary Figure S4E-F). Proteins enriched in SG conditions as compared to control were scored as SG interacting proteins (Supplementary Table 1). Gene ontology analysis shows a similar pattern of groups in different SG conditions, mainly comprised of RNA binding proteins and known SG constituents (Figure 3E, Supplementary Table 2). Interestingly, the interactome of 
PABPC1 didn't drastically change during SG formation, keeping more than $70 \%$ of its interactors (Figure 3E). Only $4.7 \%$ of the proteins (15 proteins) were present in all SG conditions and not in the control conditions (Figure 3F). The BioID SG interactome that we obtained has a significant overlap with previously determined SG component proteomes: $11.9 \%$ with FUS, 33.7\% with TDP43, 27.1\% with G3BP, and 30.2\% with SG proteome (Figure 3G, Supplementary Figure S4G; Supplementary Table 1) (Freibaum et al., 2010; Isabelle et al., 2012; Jain et al., 2016; Kamelgarn et al., 2016). We didn't find a definitive LD component, however comparing the LD proteome form HCV infected cells gave a $16.8 \%$ overlap, and LD interacting proteins were significantly enriched in our proteomics results (Figure 3J) (Khor et al., 2014; Rösch et al., 2016). It has been demonstrated that many SG components bind LDs in infection conditions (Rösch et al., 2016). Among 15 unique SG interactors that persisted across all conditions (Figure 3F), our interest was drawn to a porin: a voltage-dependent anion channel 2 (VDAC2), which is a mitochondrial outer membrane channel involved in solute transfer across the membrane (Naghdi and Hajnóczky, 2016). To confirm the interaction between SGs and VDAC2, we used bimolecular fluorescence complementation (BiFC) of VDAC2 and PABPC1 in SG conditions (Figure 3H, Supplementary Figure S4H-K) (Kerppola, 2006). We measured the increase of Venus signal in SGs over time after SG formation. Whereas the control cells did not display an increase in signal, VenusNVDAC2 showed an induction of Venus fluorescence, similar to the PABPC1-VenusN positive control BiFC interaction (Figure 3H). Additionally, we performed VDAC2 immunoprecipitation during long-term starvation (Figure 3I). Together these data indicate that SGs bind VDAC2.

\section{VDAC undergoes reversible clustering during SG formation}

VDACs display remarkable sequence conservation across species, from yeast Saccharomyces cerevisiae (Por1 and Por2) to mammalian cells (VDAC1-3) (Figure 3K; Supplementary Figure S5A-B). We took advantage of this in order to visualize the interrelationship of VDAC2, SGs, and starvation stress in live cells. In yeast $S$. cerevisiae, the highly conserved VDAC homolog, Por1, is clearly visible on the mitochondrial membrane when endogenously tagged with GFP (Figure 4A, D), and colocalizes with SGs during starvation stress (Figure 4C). Tracking yeast VDAC localization following the induction of starvation stress, we noticed that VDAC is depleted from the mitochondrial membrane, and forms inclusions on the mitochondrial surface, colocalizing with 
SGs (Figure 4A-C, D, Supplementary Figure S5C). Reversing SG formation by addition of glucose also reversed the clustering and resulted in the complete re-deployment of VDAC to the mitochondrial membrane (Figure 4D), while the amount of endogenous porin remains the same (Figure 4E). In mammalian cells as well, endogenous VDAC2 (as well as overexpressed GFPVDAC2) undergoes clustering in SG-forming conditions (Figure 4F-H, Supplementary Figure S5D, E). Over-expression of GFP-tagged VDAC2 did not provide sufficient resolution to observe VDAC2 behavior on the mitochondrial membrane, but did clearly demonstrate starvation- and arsenite-dependent clustering of VDAC2, which was dependent on SG formation and hence blocked by the addition of cycloheximide (Figure 4F, Supplementary Figure S5E). Purification of the mitochondrial fraction showed only a slight decrease in mitochondrial VDAC2 levels during SG formation, indicating that VDAC2 clusters remain on mitochondria (Supplementary Figure S5F). To further investigate VDAC2 clustering we mutated serine residues that showed particularly high conservation across species (Supplementary Figure S5G). This led us to observe that VDAC2 clustering in arsenite conditions is dependent on the residue 112S (122S in VDAC2 with N-tail, Supplementary Figure S5G). Phospho-null and phosphomimetic mutation led to a significant increase in VDAC2 clustering (Supplementary FigureS5G). Additionally, a VDAC2 knockout cell line had a reduced ability to from SGs during starvation (Supplementary Figure $\mathrm{S} 5 \mathrm{H})$. These data are consistent with the model put forth above, namely that $\mathrm{SG}$ formation triggers the inactivation of VDAC2, bringing about a re-direction of FAs to LDs, and a down-regulation of FAO. In order to reinforce this hypothesis, we investigated the requirement of VDAC2 for mitochondrial FA import.

\section{VDAC2 is essential for mitochondrial influx of fatty acids}

VDAC2 has previously been proposed to import FAs into mitochondria (Fang and Maldonado, 2018; Kerner and Hoppel, 2000; Lee et al., 2011). To study how VDAC2 affects FA trafficking we created a VDAC2 knockout using CRISPR/Cas9 gene editing (Supplementary Figure S6A). Two independent VDAC2 CRISPR/Cas9 knockouts showed a significant increase in LD formation and FA accumulation in LDs during starvation (Figure 5A-B), essentially phenocopying SG formation conditions. Complementation of full length VDAC2, but not an aggregation-prone mutant, partially reversed this phenotype (Supplementary Figure S6E). VDAC2 
localization to both mitochondria and LD-mitochondria contacts indicates a potential role in lipid flux (Supplementary Figure S6C) (Khor et al., 2014; Maurya and Mahalakshmi, 2016; Stelling et al., 2004). One possibility is that VDAC2 regulates FA import to mitochondria from LDs. To investigate this possibility, we measured LD-mitochondria contacts (Figure 5C). VDAC KO led to a decrease in mitochondria - LD contacts and accumulation of LD clusters as in SG formation conditions (Supplementary Figure S6C, D).

To further confirm the role of VDAC2 in FA trafficking, we measured FA accumulation in mitochondria during starvation in control and VDAC2 KO (Supplementary Figure S6B). We detected a significant difference between mitochondrial FA accumulation in VDAC2 KO cells and controls (Supplementary Figure S6B). To independently confirm the reduction of FA import in VDAC2 $\mathrm{KO}$ we measured FAO in VDAC2 KO, using a HADHA knockout as a negative control that is FAO-defective (Figure 5D, E). During starvation, FAO increases in control cells, while the VDAC2 knockout shows a pronounced decrease in FAO dependency, comparable to the inhibition of FA import into mitochondria (Figure 5E). During long-term starvation, however, VDAC KO FAO dependency doesn't differ from that of control cells, indicating that FAO is downregulated in the control conditions (Figure 5D).

To determine whether FAs are imported directly into mitochondria during FAO increase we performed GC-FID FA profiling in control and short-term starvation conditions in wild-type and VDAC2 KO purified mitochondria (Figure 5F, Supplementary Figure S6G). We found a consistent increase in saturated FA ratios in mitochondria during short-term starvation, but not in VDAC2 KO mitochondria. To confirm that the increase is due to FA import we performed this analysis while inhibiting FA mitochondrial import. Inhibition led to a decrease in FAs inside mitochondria (Figure $5 \mathrm{G}, \mathrm{H}$, Supplementary Figure S6G). Overall, these data point to a function of VDAC2 in mitochondrial import of FAs for FAO. To confirm that VDAC2 can directly transfer FAs across a membrane we reconstituted VDAC2 on liposomes (as was previously described in (Shao et al., 1996), followed by extrusion) (Figure 5I, J, Supplementary Figure S6H, I)). We tried to mimic mitochondrial outer membrane composition by addition of cardiolipin to the set of membrane lipids. To confirm membrane localization, we labeled VDAC2 with $\mathrm{Cy} 3$ prior to liposome formation (Figure 5J). We then measured FA import into liposomes (Figure 5K). VDAC2-containing liposomes retained significantly more FAs after washing and pelleting at 
$100000 \mathrm{~g}$ for $35 \mathrm{~min}$. Next, we added purified SGs ((Wheeler et al., 2017), Figure 5L, Supplementary Figure S6J) to VDAC2 containing liposomes and observed coaggregation of VDAC2-Cy3 with SGs. Together these data indicate that SGs downregulate FAO by inducing the reversible clustering of the VDAC2 mitochondrial importer.

\section{Stress Granule mediated signaling regulates VDAC clustering and fatty acid oxidation}

SG formation is mechanistically linked to mTOR signaling, in what is thought to be a positive feedback loop (Heberle et al., 2015; Sfakianos et al., 2018; Takahara and Maeda, 2012; Thedieck et al., 2013). mTOR inactivation leads to SG formation, which in turn recruits mTOR to SGs, which is thought to further promote mTOR inactivation ((Sfakianos et al., 2018; Takahara and Maeda, 2012), Figure 6A). Starvation stress is a common trigger for mTOR inhibition, resulting in a decrease in protein translation and in SG formation ((Sabatini, 2017), Figure 6B, Supplementary Figure S7A). mTOR inactivation happens during long term starvation, with kinetics coinciding with SG formation (Figure 6B). We confirmed that starvation induced SGs recruit inactive mTOR (Figure 6C, (Sfakianos et al., 2018; Takahara and Maeda, 2012)). The inhibition of the Akt-mTOR kinase axis is known to activate GSK3 kinase, which is implicated in VDAC2 phosphorylation ((Das et al., 2008; Hermida et al., 2017; Zhang et al., 2006), Figure 6A, D). Indeed, long term starvation, and TOR inhibition, results in activation of GSK3 (Figure 6D). Since VDAC2 is a known target of GSK3 (Das et al., 2008; Martel et al., 2013), we confirmed that clustered VDAC2 is phosphorylated (Figure 6E) and that clustering is dependent on GSK3 activity (Figure 6G, S7D). Inhibition of GSK3 with either of two selective inhibitors, BIO and SB-415286, abolishes VDAC2 clustering in response to SG formation treatment (Figure 6F-G, Supplementary Figure S7C). Strikingly, GSK3 inhibition also abolished SG formation (Figure 6F-G). As expected, by preventing VDAC2 phosphorylation, GSK3 inhibition resulted in a marked increase in FAO during long-term starvation (Figure 6I), reinforcing our model of a SG-GSK3-VDAC2 regulatory pathway for lipid flux and FAO (Figure 6J). To further confirm the connection between

SG formation and FAO down-regulation (Figure 6J), we screened a number of compounds for the ability to induce SG formation. We identified several novel SG inducers (Supplementary Figure S7D, manuscript in preparation). Remarkably, all of the SG-inducing compounds significantly 
hampered FAO up-regulation during short term starvation (Figure 6H). Together our data indicate a regulatory connection between SG formation and FAO down-regulation.

\section{ALS patient-derived iPSCs differentiated along neuronal lineage exhibit impaired FAO inhibition during long-term starvation}

To test the validity of our model in a physiological context we examined cells derived from an ALS patient with a heterozygous mutation in a SG component, TDP43, compared to a healthy matched control. The TDP43 mutant cells also showed an SG-formation defect during starvation, similarly to our CRISPR-generated TDP43 Q331K cells (Figure 7C, D, Supplementary Figure 2E). First, we differentiated iPSCs along a neuronal lineage in order to implement a starvation protocol without depriving cells of stem cell maintenance factors (Figure 7 A, B, Supplementary Figure S8 A, B). Patient-derived cells showed a defect in LD accumulation during starvation, again in similarity to our TDP43 Q331K cell line, Figure 7E, Supplementary Figure S8C). Finally, we confirmed the failure of TDP43-mutant cells to down-regulate FAO during long-term starvation. Here too, the pattern of the FAO dependency graph was in concert with what had been observed using cell lines, exhibiting a delayed inhibition response in the mutant patient-derived cells (Figure 7F). Although far from a comprehensive survey of ALS patient-derived tissues, these iPSC data provide substantial reinforcement to our model because they represent a completely independent validation of the coinciding cell biological phenotype (failure to form SGs) with the metabolic behavior (failure to down-regulate FAO) in cells derived form an individual with ALS. Taken together, these and above data support a model whereby SGs are directly involved in metabolic regulation in response to starvation stress.

\section{Discussion}

Adapting to stress requires compartmentalizing multitude out-of-equilibrium reactions in distinct organelles (Gottschling and Nystrom, 2017; Moldavski et al., 2015) and membraneless sub-domains (Amen and Kaganovich, 2015; Kaganovich, 2017; Kaganovich et al., 2008; Maharana et al., 2018), and buffering metabolic output with multi-tiered, overlapping regulatory networks, in which the nodes regulate processes at the transcriptional, translational, and post- 
translational levels (He et al., 2013; Hunter, 2009; Smart et al., 2008). We report a novel function for one type of membraneless organelle, the $\mathrm{SG}$, as a master-regulators of FAO and lipid storage during long-term starvation. In starvation conditions cells profoundly alter lipid metabolism, upregulating FAO (Douglas et al., 2016; Liu et al., 2018; Mihaylova et al., 2018). It has been unclear, however, how fatty acid trafficking is partitioned between mitochondrial import for FAO and storage in the LDs. We found that SGs direct FAs to LDs, resulting in a marked decrease of FAs imported into mitochondria for FAO at any given time. The lower rate of FAO allows cells to ration lipid resources for longer periods of starvation and has the added effect of dramatically reducing ROS production. The latter result in particular is consistent with previous reports showing that SGs are triggered by ROS (Kato et al., 2019) and help manage oxidative stress (Takahashi et al., 2013).

To further validate this model, we used genomically-edited mutants of a SG component, TDP43, to investigate the effect of a physiological perturbation of SG properties on cellular metabolism during starvation stress. Mutations in TDP43, as well as many other SG component proteins, cause heritable forms of ALS, a disease characterized by SG dysfunction, wide-spread death of motor neurons, and severe metabolic dysregulation at the cellular and organismal levels (Cleveland and Rothstein, 2001; Dupuis and Loeffler, 2009; Kim et al., 2013; Li et al., 2013a; Ling et al., 2010; Mackenzie et al., 2017). The behavior of the ALS-associated TDP43 mutant Q331K was consistent with our model of the role of SGs in regulating metabolic adaptation and specifically FAO during starvation stress. TDP43 mutant cells had a lower level of SG formation during starvation (Supplementary Figure S2E), possibly due to off-pathway aberrant aggregation of TDP43 (Gordon et al., 2019; Orru et al., 2016), or increased stability of mutant TDP43 in the nucleus, as has been previously reported (Ling et al., 2010). As a result, we observed TDP43 mutant cells continue to have increased levels of FAO than WT cells over long periods of starvation. This gives TDP43 mutant cells higher levels of ATP in the long-term starvation, as well as significantly higher levels of oxidative stress. Inhibiting FAO using the drug etomoxir reduced the ATP/ADP ratios and the ROS levels in TDP43 mutant cells. These data were consistent with our model in which SGs recruit the FA channel VDAC2 and downregulate the flux of lipids into mitochondria, thereby reducing ROS and enabling a scare resource to last for longer. Interestingly, VDAC2 levels are affected in the cellular models of ALS, which together with our results suggests 
that VDAC2 should be examined more closely as an ALS biomarker and therapeutic target (Fukada et al., 2004; Naghdi and Hajnóczky, 2016).

We propose a mechanism for the regulation of lipid flux by SGs (Figure 6J). SG formation results in the depletion of VDAC2 from the mitochondrial membrane, leading to the inhibition of FAO. SGs are known to regulate the function of nutrient sensing kinases that regulate metabolism, as has been shown previously (Arimoto et al., 2008; Kobayashi et al., 2012; Takahara and Maeda, 2012; Thedieck et al., 2013). In particular mTORC1 activity is regulated by SGs (and vice versa) (Jevtov et al., 2015; Sfakianos et al., 2018; Takahara and Maeda, 2012; Thedieck et al., 2013). We observe that starvation induced SGs retain inactive mTORC1 (Figure 6C). We propose that this creates a local depletion in mTORC1 function, leading to the activation of GSK3, which phosphorylates VDAC2 and alters mitochondrial permeability (Martel et al., 2013).

Investigating the BioID SG interactome and comparing it to previously published SG proteomic studies, uncovered a candidate channel, VDAC2, that localizes to mitochondrial outer membrane, and undergoes GSK3-dependent clustering during SGs formation. VDAC2 clustering is conserved from yeast to mammalian cells and results in decrease of membrane localized VDAC. VDAC2 has previously been proposed to function as a regulator of FA import into mitochondria, and its expression is more abundant in cells that rely more heavily on FAO (Fang and Maldonado, 2018; Kerner and Hoppel, 2000; Naghdi and Hajnóczky, 2016), including cells experiencing shortterm starvation stress (Supplementary Figure S7B). We confirmed a direct association between SG components and VDAC2, and showed that knocking down VDAC2 expression phenocopies the effect of SG formation, namely, LD biogenesis and reduction of FA import into mitochondria for FAO. Thus, we propose that induction of VDAC2 clustering by SGs blocks FA import into mitochondria, reducing FAO, and redirecting FAs towards LDs during stress. There is another possibility of VDAC2 regulation of FA trafficking. Initially reported as a mitochondrial outer membrane solute carrier, VDAC2 was later pulled down in LD proteomes (Khor et al., 2014; Rösch et al., 2016). Thus, LDs maybe interacting with mitochondria through VDAC2, and transferring FAs for FAO. In support of this, we show that VDAC2 KO results in LD clustering and reduced mitochondria-LD contacts. Recruitment of VDAC2 by SGs would therefore "trap" FAs in LDs. Additionally, recent studies have shown that VDAC2 can act as a pro-apoptotic factor (Chin et al., 2018; Lauterwasser et al., 2016; Naghdi and Hajnóczky, 2016). The recruitment of 
VDAC2 by SGs would therefore delay apoptosis, which would be consistent with another proposed SG function, namely apoptosis regulation (Arimoto et al., 2008). And finally, VDAC carries various solutes across mitochondrial membrane, thus FAO rates during long-term starvation may be reduced through the inhibition of transfer of additional metabolites.

Overall, our data lead us to propose a model in which SG formation in the vicinity of the mitochondrial membrane acts as both the "gas" and the "breaks" of lipid metabolism during stress: directing FA uptake and trafficking, while inhibiting FAO itself sufficiently to ensure cells do not run out of their backup fuel source and minimizing the damage arising from utilizing it instead of glucose. In order to regulate metabolic flux SGs inhibit mTOR signaling while activating the GSK3 pathway (Hermida et al., 2017; Thedieck et al., 2013). This leads to phosphorylation and clustering of VDAC2, resulting in reduced mitochondrial permeability for FAs and their subsequent accumulation in LDs (Sengupta et al., 2010). The reversible clustering of VDAC may serve additional purposes during starvation and stress, since VDACs are permeable to multiple cellular metabolites in addition to FAs and regulate calcium homeostasis, oxidative stress, and apoptosis (Shoshan-Barmatz et al., 2010). SG-dependent VDAC clustering demonstrates how SG signaling pathways can induce multi-tiered architectural changes in the cell, affecting metabolism and cellular fate. As recent findings suggest, SG can form on the surface of membrane bound organelles or cytoskeletal components regulating cellular functions such as, kinase signaling, altering local translation (Cioni et al., 2019; Lee et al., 2020; Liao et al., 2019; Lin et al., 2016) or enhance the transcriptional response to starvation (manuscript in preparation); here we show SGs regulating mitochondrial function by altering local permeability.

This model of SG function has several important implications. Many recent studies have pointed to the contribution of metabolic dysregulation, particularly lipid and LD metabolism, in human neurodegenerative diseases including ALS (Asanuma et al., 2011; Fukada et al., 2004; Pennetta and Welte, 2018; Szelechowski et al., 2018; Tefera and Borges, 2017; Vandoorne et al., 2018). Our data establish a mechanistic connection between aberrant SG formation, which is another hallmark of ALS, and the metabolic abnormalities observed in the disease (Li et al., 2013a). Using ALS patient-derived stem cells with a mutation in a SG component, we confirm out model of delayed FAO inhibition in the long-term starvation. ROS accumulation, which is observed in ALS-affected neurons, may result from aberrant FAO regulation as well (Barber and 
Shaw, 2010; Knobloch et al., 2017; Pollari et al., 2014). A recent report indicating a role for SGs in mediating ROS damage is therefore consistent with the idea that SGs downregulate FAO (Takahashi et al., 2013). Additional investigation of how SGs modulate VDAC2 and lipid flux in different cell types will be the next important step in understanding their role in starvation stress response.

\section{Acknowledgments}

We thank Maayan Kaganovich for comments on the manuscript. We thank the Outeiro Lab for support with essential equipment as well as Christof Lenz, Lisa Neuenroth, and Thierry Wasselin at the UMG Core Facility Proteomics for obtaining MS data. William Breuer provided technical assistance with Mass Spectrometry sample preparation. We thank Vitas Analytical Services (vitas.no) and Tore Bolstad for GC-FID data acquisition. This work was supported by the European Research Council under the European Union's Seventh Framework Program (FP/20072013)/ERC-StG2013 337713 DarkSide starting grant. TA was funded by the Jerusalem Brain Community (JBC) Doctoral Fellowship during part of this project. The authors declare no conflicts of interest.

\section{Experimental Procedures}

\section{Cell culture and cell lines}

HEK293T cells were maintained in high glucose DMEM supplemented with $10 \%$ fetal bovine serum (FBS), $1 \%$ penicillin/streptomycin, at $37^{\circ} \mathrm{C} / 5 \% \mathrm{CO} 2$, SH-SY5Y cells were maintained in high glucose 1:1 F12/DMEM media supplemented with 10\% FBS, 1\% penicillin/streptomycin at $37^{\circ} \mathrm{C} / 5 \% \mathrm{CO}$. Cells modified via CRISPR/Cas9 were maintained as above with addition of puromycin $(2 \mu \mathrm{g} / \mathrm{ml}$, Sigma) during selection of clonal populations. Patient-derived iPSCs (CedarSinai) were maintained in mTeSR ${ }^{\mathrm{TM}} 1$ (STEM CELL Technologies) media on Matrigel-coated plates. Differentiation to neural progenitor cells was done using STEMdiff ${ }^{\mathrm{TM}}$ neural induction protocol (STEM CELL Technologies, with addition of SMADi). Neural progenitor cells were maintained in STEMdiff ${ }^{\text {TM }}$ neural progenitor medium (STEM CELL Technologies). 


\section{Starvation protocol}

Cells were grown to $70-90 \%$ confluency. After PBS wash, media was replaced with freshly prepared DMEM media without FBS, glucose, pyruvate, L-glutamine, and with $0.25 \mathrm{~g} / 100 \mathrm{ml}$ fatty acid free bovine serum albumin (BSA). Cells were incubated in the starvation medium for indicated amounts of time, the viability was confirmed by microscopy. D-glucose $(4 \mathrm{~g} / \mathrm{L}), \mathrm{L}-$ glutamine $(2 \mathrm{mM})$, and synthetically defined lipid mixture $1(1 \mu \mathrm{l} / 10 \mathrm{ml}$ media, Sigma) were added to the media in control conditions.

Natural starvation was performed by incubating $90 \%$ confluent cells for 4 days without changing the medium (Figure S1K).

\section{Lipid import into LDs in live cells:}

Lipid droplets are pre-stained with either Bodipy (Green, $1 \mu \mathrm{M}$ Thermo Fischer Scientific) or Bodipy-C12 (Red, $1 \mu \mathrm{M}$ Thermo Fischer Scientific) for 30min. Fatty acids (bodipy-C12, 1 $\mu \mathrm{M}$ ) or Cholesterol (Bodipy-cholesterol, Green, $1 \mu \mathrm{M}$ ) were added at the start of the acquisition (HolttaVuori et al., 2008). Lipid droplets were monitored in live cells every 5 seconds for 15 min using Nikon A1r confocal microscope. Accumulation of lipid in lipid droplets was measured as an increase of fluorescent intensity of a corresponding lipid-bodipy fusion. Fluorescence intensity was quantified inside lipid droplet area in 30 cells.

\section{Antibodies}

We used the following reagents to detect proteins: monoclonal anti-G3BP (Sigma-Aldrich WH0010146M1), polyclonal anti-TIA1 produced in rabbit (Sigma-Aldrich SAB4301803), antiGAPDH (sc-47724, Santa Cruz Biotechnology), anti-DDR2 (Origene, clone 1G6), anti PPARA (sc 398394, Santa Cruz Biotechnology), anti-PPARG (sc-7273X, Santa Cruz Biotechnology), antiPPARD (sc74517, Santa Cruz Biotechnology), anti-mCherry (34974 Invitrogen), anti phospho4EBP (Thr46, 1170G Invitrogen), anti-4EBP (sc 9977, Santa Cruz Biotechnology), anti-HADHA (sc-374497, Santa Cruz Biotechnology), anti-Tom22 (sc58308, Santa Cruz Biotechnology), antiVDAC2 (PA528106, Invitrogen), and anti-TARDBP (sc-376532, Santa Cruz Biotechnology), anti-VDAC1 antibody (sc-390996, Santa Cruz Biotechnology), anti-mTOR (sc-517464, Santa Cruz Biotechnology), anti GSK3 alpha (D80E6) (4337, Cell Signaling Technology), anti-phospho- 
GSK3-alpha (36E9, Ser21) (9316, Cell Signaling Technology), anti phospho-Serine (ab9332, Abcam).

Secondary antibodies for immunofluorescence: anti-Rabbit IgG Cy3-conjugated (Sigma-Aldrich C2306), anti-Mouse IgG Cy3-conjugated (Sigma-Aldrich C2181), anti-rabbit IgG Cy5 conjugated (Invitrogen A10523).

\section{Chemicals}

BODIPYтм 558/568 C12 (4,4-Difluoro-5-(2-Thienyl)-4-Bora-3a,4a-Diaza-s-Indacene-3Dodecanoic Acid, Thermo Fischer Scientific), Mito Red 569/594 (Sigma), Hoechst (Sigma), Rhodamine800 (Sigma), sodium arsenite (Fischer Chemical), cycloheximide (Sigma), BODIPY тм 493/503 (ThermoFischer Scientific, D3922), Bodipy FL Cholesterol (Setareh Biotech), Streptavidin-Cy3 (Thermo Fischer Scientific), fatty acid free BSA (PAN), Lipid Mix (Sigma), UK5099 (Sigma), Etomoxir (Sigma), BPTES (Sigma), Tetramethylrhodamine ethyl ester perchlorate (TMRE, Sigma), 2',7'-Dichlorofluorescin diacetate (H2-DFCDA, Sigma), 2bromopalmitic acid (2-BP, Sigma), Rotenone (Sigma), CCCP (Sigma), Streptavidin-HRP (Thermo Scientific), fatty acid free BSA (PAN Biotech), DMEM (PAN Biotech), FBS (PAN Biotech), PBS (PAN Biotech), vanillin (Sigma), methanol (Roth), Chlorophorm (Sigma), FAMdC-Puro, Jena Bioscience, biotin (Sigma), aprotinin (Roth), leupeptin (Roth), Phenylmethylsulfonyl fluoride (PMSF, Sigma), erastin (571203-78-6, Cayman Chemical), SMI4a (438190-29-5, Cayman chemical), BIO (667463-62-9, Cayman chemical), SB-415286 (26421823-7, Cayman chemical), 1-NA-PP1 (Cayman chemical), AS-703026 (Cayman chemical), ruxolitinib (Cayman chemical), KN-93 (Cayman chemical), TGX-221 (Cayman chemical), AG17 (Cayman chemical).

\section{CRISPR/Cas9}

Knockout and endogenously tagged cell lines were constructed using CRISPR/Cas9 protocol and plasmids described in Ran et. al (Ran et al., 2013). Knockout cell lines were verified by western blotting, immunofluorescence. Genomic DNA was sequenced to verify disrupted region in knockout or fidelity of endogenous tagging. Functional assay for knockout verification was performed where applicable (FAO dependency for HADHA knockout). Endogenous tagging was performed by fusing tagging construct (linker-GFP/DDR2/mCherry/mCherry-VenusC-polyA- 
Puromycin) to the region upstream of the stop codon $(\sim 500 \mathrm{bp})$ and downstream of stop codon ( $\sim 500 \mathrm{bp})$. PCR product containing homologous regions flanking the tagging construct was cotransfected with px330-gRNA corresponding construct. Endogenous tagging was verified by western blotting, immunofluorescence staining, and genomic DNA sequencing. CRISPR specificity was profiled using Digenome-Seq web tool (http://www.rgenome.net/cas-offinder/) (Bae et al., 2014). Off targets were found for TDP43 endogenous tag, however the off-target was found within TDP43 pseudogene2 (13:60275990), thus wasn't pursued. The following target sequences are used to modify genomic DNA: endogenous tagging of PABPC1 'TTAAAGTGAGCTTTTCCCTC', endogenous tagging of HADHA 'CCTAACAAGAAGTTCTACCAGTGAGC', knockout of HADHA 'CTGCTGTCCTCTTCAGCTCAAGATGG', knockout of VDAC2 'TAAGTAAAGCTGGGATCTCTGCGGGA', tagging of 'GTCTTCTGGCTGGGGAATGTAGACAG'.

\section{Plasmid Construction:}

All plasmids were constructed using Escherichia coli strain DH5 $\alpha$. Plasmids used in this study are summarized in the Table 1. B4GALT182 fragment was cloned from mIFP-Golgi-7, which was a gift from Michael Davidson (Addgene plasmid \# 56221; http://n2t.net/addgene:56221; RRID:Addgene_56221) (Yu et al., 2015). We used px459 plasmid to clone CRISPR/Cas9 constructs for gene knockout, and px330 plasmid to construct plasmids used for endogenous tagging. pSpCas9(BB)-2A-Puro (PX459) V2.0 was a gift from Feng Zhang (Addgene plasmid \# 62988; http://n2t.net/addgene:62988; RRID:Addgene_62988) (Ran et al., 2013). pX330-U6Chimeric_BB-CBh-hSpCas9 was a gift from Feng Zhang (Addgene plasmid \# 42230; http://n2t.net/addgene:42230; RRID:Addgene_42230) (Cong et al., 2013). GW1-PercevalHR was a gift from Gary Yellen (Addgene plasmid \# 49082; http://n2t.net/addgene:49082; RRID:Addgene_49082) (Tantama et al., 2013). pCI-MS2V5-PABPC1 was a gift from Niels Gehring (Addgene plasmid \# 65807; http://n2t.net/addgene:65807; RRID:Addgene_65807) (Fatscher et al., 2014). Site directed mutagenesis was performed to obtain VDAC2 mutants. Plasmid maps and cloning information is available upon request.

\begin{tabular}{|l|l|}
\hline Plasmid name & Source \\
\hline pcDNA3.1 $\mathrm{mCH}^{*} 4 \mathrm{skl}$ & This study \\
\hline
\end{tabular}




\begin{tabular}{|c|c|}
\hline pcDNA3.1 hLAMP1-mCH & This study \\
\hline $\begin{array}{l}\text { pcDNA3.1 CALR } \\
\text { mCHkdel }\end{array}$ & This study \\
\hline pcDNA3.1 Golgi-mCH & $\begin{array}{l}\text { Cloned from } \text { Addgene } \\
\text { plasmid } \# 56221\end{array}$ \\
\hline $\begin{array}{l}\text { pUC19-5'PABPC1-DDR2- } \\
\text { puro-3'PABPC1 }\end{array}$ & This study \\
\hline Px330-PABPC1-gRNA & This study \\
\hline GW1-PercevalHR & Addgene plasmid \#49082 \\
\hline $\begin{array}{l}\text { pUC19-5'PABPC1-mCH- } \\
\text { puro-3'PABPC1 }\end{array}$ & This study \\
\hline Px459-HADHA-KO-gRNA & This study \\
\hline Px330-HADHA-gRNA & This study \\
\hline $\begin{array}{l}\text { pUC19-5'HADHA-GFP- } \\
\text { puro-3'HADHA }\end{array}$ & This study \\
\hline Px330-VDAC2-KO-gRNA & This study \\
\hline Px459-TDP43-gRNA & This study \\
\hline $\begin{array}{l}\text { pUC19-5'TDP43-Q331K- } \\
\text { GFP-puro-3'TDP43 }\end{array}$ & This study \\
\hline $\begin{array}{l}\text { pcDNA-PABPC1- } \\
\text { BirA(R118G)-GFP }\end{array}$ & $\begin{array}{l}\text { This study, PABPC1 from } \\
\text { addgene plasmid } \# 65807\end{array}$ \\
\hline pEC1-VenusN-VDAC2 & $\begin{array}{l}\text { This study, using pEGFPC1 } \\
\text { backbone }\end{array}$ \\
\hline pcDNA3.1- VenusN & This study \\
\hline pcDNA3.1-PABPC1-VenusN & This study \\
\hline $\begin{array}{l}\text { pUC19-5'PABPC1-mCherry- } \\
\text { VenusC-puro-3'PABPC1 }\end{array}$ & This Study \\
\hline pEGFPC1-VDAC2 & This study \\
\hline pEGFPC1-VDAC2 S112A & This study \\
\hline pEGFPC1-VDAC2 S112D & This study \\
\hline
\end{tabular}




\begin{tabular}{|l|l|}
\hline $\begin{array}{l}\text { pEGFPC1-VDAC2 } \\
\text { S117/118AA }\end{array}$ & This study \\
\hline pEGFPC1 & Clontech \\
\hline pKT127-GFP-HPH & This study \\
\hline pKT127-mCH-KAN & This study \\
\hline
\end{tabular}

\section{ATP/ADP ratiometric imaging}

We used live cell ATP/ADP ratio sensor PercevalHR to measure the ATP/ADP ratio changes (Tantama et al., 2013). Cells were imaged with a confocal Nikon A1r microscope equipped with a cell culture environmental chamber maintaining $5 \% \mathrm{CO} 2 / 95 \%$ air gas mixture, $37^{\circ} \mathrm{C}$, using a 60x PlanApo VC oil objective NA 1.40. PercevalHR was excited using 406nm and 488nm lasers (OBIS), emission was collected through a 525/50 nm band pass filter. Images were obtained every $30 \mathrm{~min}$ during $6 \mathrm{~h}$. Rotenone was added to a negative control sample after first time loop at a $4 \mu \mathrm{M}$ final concentration. Images were processed using NIS-Elements Software (Nikon). ATP/ADP ratios were calculated relative to a positive control as described in (Tantama et al., 2013).

When using CRISPR/Cas9 tagged PABPC1-mCH together with PercevalHR, ratiometric and normal images were obtained sequentially.

\section{Total lipid and free fatty acid quantification}

Free fatty acids were analyzed according to instructions using Free Fatty acid quantification kit (Sigma). Total lipid extraction and quantification was performed using sulfo-phospho-vanillin method (Frings et al., 1972). Lipids were extracted by a modified Folch method (Folch et al., 1957). Briefly, cell pellet was mixed in 8:8:3 methanol:water:chloroform, centrifuged $12 \mathrm{~g}$ for $10 \mathrm{~min}$, the lower phase was collected and air dried.

\section{Glycerol release and TAG quantification}

Glycerol in supernatants and TAG were analyzed according to instruction using adipolysis and adipogenesis kits (Sigma). Glycerol was quantified in supernatants; the media was changed 1 hour prior to the measurement to a starvation media ( $1 \mathrm{ml}$ per well in a 6-well plate). TAG was measured 
after the addition of Lipid Mix mix 1 (Sigma) containing $2 \mu \mathrm{g} / \mathrm{ml}$ arachidonic, $10 \mu \mathrm{g} / \mathrm{ml}$ linoleic, linolenic, myristic, oleic, palmitic and stearic, and $0.22 \mathrm{mg} / \mathrm{ml}$ cholesterol $(1 \mathrm{ul} / \mathrm{ml})$.

\section{Fatty acid oxidation dependency}

Seahorse XFe96 Flux Analyzer (Agilent) was used to measure fatty acids metabolic dependency. Cells were seeded on 96-well plate (Agilent), starvation was done for indicated amounts of time as described above, fatty acid dependency in live cells was measured according to Mito Fuel Test kit (Agilent).

\section{Oxidative stress assessment}

Cells were incubated with $100 \mu \mathrm{M} 2$ ', 7'-dichlorodihydrofluorescein diacetate (H2-DFCDA) for $30 \mathrm{~min}$ prior to imaging. Fluorescence intensity (excitation with $488 \mathrm{~nm}$ ) was measured in the cytoplasm of single cells ( $\mathrm{n}=30)$ using NIS software (Nikon).

\section{Measurement of active translation}

Cells were incubated with $0.5 \mu \mathrm{M}$ 6-FAM-dC-Puromycin (FAM-dC-Puro, Jena Bioscience) for 6 hours, fluorescence intensity was measured in the cytoplasm of single cells $(n=30)$, at 0,3 , and 6 hours in indicated conditions. Incorporation of puromycin was considered a readout of active translation according to (Starck et al., 2004).

\section{Extraction of biotinylated proteins}

Cells expressing PABPC1-BirA(R118G)-GFP were grown to 80-90\% confluency. SGs were induced with $100 \mu \mathrm{M}$ arsenite, $200 \mu \mathrm{M}$ 2-BP, 9-hour starvation, or PPAR activation with $100 \mu \mathrm{M}$ rosiglitazone, clofibrate, and GW601516. After that, cells were incubated with biotin $(100 \mu \mathrm{M})$ for 4hours, SGs formation was visualized on the microscope. Lysis and affinity capture were done according to Roux et al. with minor modifications (Roux et al., 2012). Cells were washed with PBS and (subsequent steps at $4^{\circ} \mathrm{C}$ ) lysed in the buffer $(50 \mathrm{mM}$ Tris $\mathrm{pH} 7.4,500 \mathrm{mM} \mathrm{NaCl}$, 0.4\%SDS, 5mM EDTA, 1mM DTT, and protease inhibitor cocktail (aprotinin, leupeptin, and PMSF, $10 \mu \mathrm{g}$ ) with glass beads $425-600 \mu \mathrm{m}$ (Sigma). After $1 \mathrm{~min}$ of vortex Triton X-100 was added to $2 \%$ concentration, after second round of vortex equal amount of $50 \mathrm{mM}$ Tris $\mathrm{pH} 7.4$ was added. After third round of vortex and centrifugation 5min $13000 \mathrm{rpm} 300 \mu \mathrm{l}$ of streptavidin-coated magnetic beads (NEB) were added to collected supernatants and incubated overnight at $4^{\circ} \mathrm{C}$ with 
agitation, $10 \%$ of the sample was collected for the Western Blot analysis. Beads were collected and (subsequent steps at room temperature) washed according to Roux et al.

\section{Preparing samples for the Mass spectroscopy}

The beads were washed free of detergents by two washes with $25 \mathrm{mM}$ Tris-HCl pH 8.0. Then the packed beads were resuspended in $100 \mathrm{ul}$ of $8 \mathrm{M}$ urea, $10 \mathrm{mM}$ DTT, $25 \mathrm{mM}$ Tris-HCl pH 8.0 and incubated for $20 \mathrm{~min}$, followed by addition of iodoacetamide to a concentration of $55 \mathrm{mM}$ and incubation for $20 \mathrm{~min}$ in the dark. The urea was diluted by the addition of 6 volumes of $25 \mathrm{mM}$ Tris- $\mathrm{HCl} \mathrm{pH} 8.0,0.25 \mu \mathrm{g}$ trypsin was added (Sigma) and the beads were incubated overnight at $37^{\circ} \mathrm{C}$ with gentle agitation. The released peptides were desalted by loading the whole bead supernatant on C18 Stage tips (Rappsilber et al., 2007). Eluted peptide material was used for MS analysis.

\section{LC-MS/MS analysis}

Protein digests were analyzed on a nanoflow chromatography system (Eksigent nanoLC425) hyphenated to a hybrid triple quadrupole-TOF mass spectrometer (TripleTOF 5600+) equipped with a Nanospray III ion source (Ionspray Voltage $2400 \mathrm{~V}$, Interface Heater Temperature $150^{\circ} \mathrm{C}$, Sheath Gas Setting 12) and controlled by Analyst TF 1.7.1 software build 1163 (all AB Sciex). In brief, peptides were dissolved in loading buffer ( $2 \%$ acetonitrile, $0.1 \%$ formic acid in water), enriched on a precolumn $(0.18 \mathrm{~mm}$ ID x $20 \mathrm{~mm}$, Symmetry C18, $5 \mu \mathrm{m}$, Waters, Milford/MA, U.S.A) and separated on an analytical RP-C18 column (0.075 mm ID x 250 mm, HSS T3, $1.8 \mu \mathrm{m}$, Waters) using a $90 \mathrm{~min}$ linear gradient of 5-35\% acetonitrile/0.1\% formic acid (v:v) at $300 \mathrm{nl} \mathrm{min}^{-}$ 1 .

Qualitative LC-MS/MS analysis was performed using a Top25 data-dependent acquisition method with an MS survey scan of m/z 350-1250 accumulated for $350 \mathrm{~ms}$ at a resolution of 30,000 full width at half maximum (FWHM). MS/MS scans of m/z 180-1600 were accumulated for $100 \mathrm{~ms}$ at a resolution of 17,500 FWHM and a precursor isolation width of $0.7 \mathrm{FWHM}$, resulting in a total cycle time of 2.9 s. Precursors above a threshold MS intensity of $125 \mathrm{cps}$ with charge states 2+, $3+$, and 4+ were selected for MS/MS, the dynamic exclusion time was set to $30 \mathrm{~s}$. MS/MS

activation was achieved by CID using nitrogen as a collision gas and the manufacturer's default rolling collision energy settings. Two technical replicates per sample were acquired. 


\section{MS data analysis}

Mass spectra data were processed using the MaxQuant computational platform, version 1.6.3.4 (Cox and Mann, 2008). Peak lists were searched against the human Uniprot FASTA sequence database (downloaded 02.01.19). The search included cysteine carbamidomethylation as a fixed modification and oxidation of methionine as variable modifications. Peptides with minimum of seven amino-acid length were considered and the required FDR was set to $1 \%$ at the peptide and protein level. Protein identifications required at least three unique or razor peptides per protein group. Relative protein quantification in MaxQuant was performed using the label free quantification (LFQ) algorithm (Cox et al., 2014). Identified proteins were analyzed with Perseus software (Tyanova et al., 2016). Gene ontology analysis was performed using STRING web tool (https://string-db.org/,(Szklarczyk et al., 2015).

\section{GC-FID}

Fatty acid profiles were done in purified mitochondria. 50 million cells were used to prepare one sample. Mitochondria were purified using purification kit for cultured cells (Thermo Fischer Scientific).

Purified mitochondria were vortexed and pipetted into vials. Samples were methylated with 3N $\mathrm{HCl}$ in Methanol. FAMEs were extracted with hexane, then samples were neutralized with $3 \mathrm{~N}$ $\mathrm{KOH}$ in water. After mixing and centrifuging the hexane phase was injected into the GC-FID. Analysis was performed on an $8890 \mathrm{GC}$ with a split/splitless injector, a 7693A automatic liquid sampler, and flame ionization detector (Agilent Technologies, Palo Alto, CA). Separations was performed on a TR-FAME $(30 \mathrm{~m} \times 0.25 \mathrm{~mm}$ i.d. $\times 0.25 \mu \mathrm{m}$ film thickness $)$ column from ThermoFisher Scientific.

\section{Liposome preparation}

Liposomes were prepared by freeze-drying chlorophorm dissolved lipids followed by wetting and extrusion at room temperature (Szoka and Papahadjopoulos, 1980) (Avanti mini extruder set, Sigma). The following lipids were used to create a membrane- Phosphatidylcholine $(2 \mathrm{mg} / \mathrm{ml})$, cholesterol $(0.125 \mathrm{mg} / \mathrm{ml})$, cardiolipin $(0.125 \mathrm{mg} / \mathrm{ml})$, Phosphatidylinositol $(0.125 \mathrm{mg} / \mathrm{ml})$, L- $\alpha-$ Phosphatidylethanolamine $(0.25 \mathrm{mg} / \mathrm{ml})$, phosphatidyl serine $(0.125 \mathrm{mg} / \mathrm{ml})$. Pure VDAC2 (Abcam) was reconstituted according to Shao et al., 1996, we used solutions described in Yu et 
al., 2012. After removing the residual with brief spinning, VDAC2 incorporation was determined by pelleting liposomes with $100000 \mathrm{~g}$ for 35 minutes and quantifying VDAC2 concentration in super and pellets with western blot. To visualize VDAC2 we used Cy3 $\mathrm{Cy} 3^{\mathrm{TM}}$ Mono-Reactive Dye (Sigma), VDAC2 was labeled with Cy3 according to manufacturer's instructions.

\section{RNA preparation and real time PCR}

Total mRNA was extracted from cells using TRI Reagent (Sigma). cDNA synthesis was performed using first strand cDNA synthesis kit (NEB). Real time PCR was performed using QuantStudio3 (Thermo Fischer Scientific). mRNA levels were quantified using QuantStudio3 software. Experiments were repeated three times with 2 technical repeats and fold difference in expression was calculated by $\Delta \Delta \mathrm{Ct}$ method using GAPDH as a housekeeping gene (Livak and Schmittgen, 2001).

\begin{tabular}{|l|l|}
\hline Primers for real time PCR & Target \\
\hline 5'-GCCAAGCTCCTGAAGCAGAAG & OCT4 \\
\hline 5'-CCTCCACCCACTTCTGCAGC & OCT4 \\
\hline 5'-CTCTTCCACCCAGCTGTGTG & NANOG \\
\hline 5'-CGGCCAGTTGTTTTTCTGCC & NANOG \\
\hline 5'-GGGGAAGGTGAAGGTCGGAGTC & GAPDH \\
\hline 5'-GTGCCATGGAATTTGCCATGGG & GAPDH \\
\hline 5'-GGGCCTACAGAGCCAGATCG & NES \\
\hline 5'-CTGAAAGCTGAGGGAAGTCTTGG & NES \\
\hline 5'-GGAATCAGAGAAGACAGGCCAGC & PAX6 \\
\hline 5'-CCATGGTGAAGCTGGGCATAG & PAX6 \\
\hline
\end{tabular}

\section{Bimolecular Fluorescent Complementation (BiFC)}

We used Venus fluorescent protein complementation to assess protein-protein interaction in live cells (Nagai et al., 2002; Rackham and Brown, 2004). We inserted mCherry-VenusC fragment into genomic region of PABPC1 using CRISPR/Cas9. Next, we constructed VenusN, VenusNVDAC2, and PABPC1-VenusN plasmids. Venus complementation signal was detected in live 
cells during the course of SGs formation using confocal microscopy (excitation wavelength $488 \mathrm{~nm})$. Fluorescence intensity was quantified using NIS software (Nikon).

\section{Immunofluorescence}

Cells were grown on glass bottom plates. Cells were fixed using 4\% paraformaldehyde (with addition of $0.25 \%$ glutaraldehyde for VDAC2 immunofluorescence) for 10 minutes, followed by permeabilization with $0.5 \%$ Triton X100, then blocked overnight in 5\% BSA in PBS.

\section{Yeast strains and manipulations}

We used standard conditions for culturing yeast cells (Sherman, 2002). Yeast strains used in this study are based on BY4741 strain (Brachmann et al., 1998; Thomas and Rothstein, 1989). Yeast transformations were performed using LiAc/PEG method (Gietz et al., 1995) with minor modifications (Amen and Kaganovich, 2017). Unless otherwise specified, genes were endogenously tagged using modified pKT127 plasmids (Sheff and Thorn, 2004). Chromosomal integration was verified by PCR. Experiments were done using Synthetic Defined (SD) medium. The strains used in the study are summarized in Table 2.

Table 2

\begin{tabular}{|l|l|}
\hline Strain name & Source \\
\hline BY4741 POR1-GFP-KAN & This study \\
\hline BY4741 PAB1-GFP-HPH & This study \\
\hline BY4741 PAB1-GFP-HPH POR1-mCH-KAN & This study \\
\hline
\end{tabular}

\section{Protocol for Stress Granule induction in yeast}

Freshly grown yeast culture was diluted and grown in SD media to mid log phase. Cells are pelleted and washed twice with water and SD media without glucose, and the media is changed to SD without glucose for the time indicated in the experiment. Cells with SG are visualized in the same media, changing the media to SD with glucose will result in fast clearance of inclusions.

\section{Microscopy}


For live cell imaging we used 4-well microscope glass bottom plates (IBIDI), or Cellview cell culture dish (Greiner Bio One). Plates were coated with Concanavalin A (Sigma) for live cell imaging of yeast. Confocal images and movies were acquired using a dual point-scanning Nikon A1R-si microscope equipped with a PInano Piezo stage (MCL), temperature and CO2 incubator, using a 60x PlanApo VC oil objective NA 1.40. We used 406nm, 488nm, 561nm, and 640nm laser (Coherent, OBIS). Movies for kymographs were acquired in resonant-scanning mode. Image processing was performed using NIS-Elements software.

\section{Statistics and data analysis}

Three or more independent experiments were performed to obtain the data. $\mathrm{P}$ values were calculated by two-tailed Student t-test, or one-way ANOVA for samples with $\mathrm{N}>10$ following normal distribution. Normal distribution of the data was verified using Shapiro-Wilk test and the equality of variances was verified by Levene's test. Mann-Whitney, or Kruskal-Wallis tests were used for experiments with less than 5 samples or when samples didn't follow a normal distribution. The sample sizes were not predetermined.

Random scatter plots for organelle proximity screen were generated using Matplotlib (Hunter, 2007). Proximity of random sample was quantified by manually aligning the sample and reference image and quantifying interactions, followed by statistical analysis.

Sequence alignment and distance visualization was performed using ClustalW and Jalview tools (Sievers et al., 2011; Waterhouse et al., 2009). 


\section{Figure Legends:}

Figure 1. Stress Granule formation in starvation coincides with a decrease in fatty acid oxidation

(A) ATP/ADP ratio changes during starvation show differences in the long-term starvation. HEK293T cells expressing PercevalHR ATP/ADP ratio marker were subjected to fuel starvation for 12 hours while ratiometric imaging every 1 hour. Representative confocal planes of the ratio (ex488/ex405) are shown, scale bar $5 \mu \mathrm{m}$.

(B) Quantification of ATP/ADP ratio changes during starvation. Rotenone $4 \mu \mathrm{M}$ was added as a negative control. Graphs show normalized ATP/ADP ratio (ex488/ex405) in starvation and control conditions, mean $\pm \mathrm{SEM}, *$ indicates $\mathrm{p}<0.05$.

(C) Analysis of FA oxidation dependency using Seahorse Flux analyzer. HEK293T cells were starved in no glucose, no glutamine, or no glucose and no glutamine lipid free media for 3 hours. Then oxygen consumption rate (OCR) was measured in real time using Mito Fuel Flex Test (Agilent) to determine fuel usage. Graph represents reliance on FAs to maintain respiration, mean \pm SEM, * indicates $\mathrm{p}<0.05$.

(D) Starving cells rely on HADHA FA oxidation enzyme to maintain ATP/ADP ratio. Control and HADHA knockout (KO) HEK293T cells (refer to Figure S1B) expressing PercevalHR were starved for 12 hours while ratiometric imaging. Representative confocal planes are shown, scale bar $5 \mu \mathrm{m}$. Graph shows average ATP/ADP ratio (ex488/ex405) in starvation and control conditions, mean \pm SEM.

(E) HADHA levels increase during starvation. HEK293T cells were starved for 3 and 6 hours or treated with FAO activators - clofibrate, rosiglitazone, and GW501516 (100 $\mu \mathrm{M}$ each) for 3 hours. Western blot shows upregulation of HADHA, quantification shows fold induction of HADHA relative to control conditions (lane 2) (refer to Figure S1C-F for HADHA-GFP levels).

(F) Analysis of FAO during short-term and long-term starvation comparing to control conditions. Human SH-SY5Y cells with or without TDP43 Q331K-GFP mutation (refer to Figure S2A-C) were grown in control medium or starved for 4 or 18 hours and analyzed using Seahorse Mito Fuel Flex test. Line graph represents fatty acid dependency mean $\pm \mathrm{SEM}, *$ indicates $\mathrm{p}<0.05$. 
(G) Starving cells with TDP43 Q331K mutation maintain ATP/ADP ratio. Control and TDP43 Q331K cells expressing PercevalHR were starved for 24 hours, ratiometric imaging was performed, Hoechst was added 15 minutes prior to the imaging to enable cytoplasmic measurement. Representative confocal planes are shown, scale bar $5 \mu \mathrm{m}$. Graph shows average ATP/ADP ratio (ex488/ex405) in starvation and control conditions, mean \pm SEM, * - $\mathrm{p}<0.05$.

(H-I) Starving cells with TDP43 Q331K mutation have increased oxidative stress levels. Control and TDP43 Q331K cells expressing were starved for 24 hours, H2-DFCDA $(100 \mu \mathrm{M})$ was added 1 hour prior to the imaging. Representative confocal planes are shown, scale bar $5 \mu \mathrm{m}$. Graph shows average cytoplasmic fluorescence intensity in starvation and control conditions, mean \pm SEM, * $\mathrm{p}<0.01$.

(J) Inhibition of fatty acid import to mitochondria decreases ATP/ADP levels and oxidative stress during starvation in TDP43 Q331K mutant cells. Upper panels: cells with TDP43 Q331K mutation expressing PercevalHR were incubated in starvation media with vehicle or etomoxir $(5 \mu \mathrm{M})$. Hoechst $(10 \mu \mathrm{g} / \mathrm{ml})$ was used to stain the nucleus 30 minutes prior to imaging. Ratiometric images are shown, scale bar $5 \mu \mathrm{m}$. Lower panels: cells with TDP43 Q331K mutation were incubated in starvation media with vehicle or etomoxir $(5 \mu \mathrm{M})$ (refer to Figure S2F). H2-DFCDA $(100 \mu \mathrm{M})$ was added 1 hour prior to the imaging, scale bar $5 \mu \mathrm{m}$. Graphs show average ATP/ADP ratio (ex488/ex405) in starvation and control condition (left), and cytoplasmic fluorescence intensity in starvation and control conditions (right), mean \pm SEM, * - p $<0.05$.

(K) SG formation during starvation anti-correlates with FAO. HEK293T cells were grown in control medium or starved for 4 or 18 hours and analyzed using Seahorse Mito Fuel Flex test. Line graph represents fold change in fatty acid dependency, mean \pm SEM. Cells with CRISPR/Cas9 PABPC1-DDR2 (refer to Figure S1G-I) were starved for 18 hours, \% of cells with SGs were quantified at $0,3,6,9$ and 18 hours, mean $\pm \operatorname{SEM}$.

(L) SG formation timeline of PABPC1-DDR2. CRISPR/Cas9 tagged PABPC1-DDR2 cells were incubated in fuel starvation media for indicated amount of time (refer to Figure S1J-M). Representative confocal planes are shown, arrows indicate SGs, scale bar $5 \mu \mathrm{m}$.

(M) Imaging of ATP/ADP ratio during SG formation. PABPC1-mCH cells (refer to Figure S1NP) expressing PercevalHR were treated with either arsenite $(100 \mu \mathrm{M})$ for 1 hour, or starved for 12 
hours, or treated with rotenone $(4 \mu \mathrm{M})$ and $\mathrm{CCCP}(10 \mu \mathrm{M})$ for 1 hour. Representative images are shown, scale bar $5 \mu \mathrm{m}$.

Figure 2. Stress Granule formation promotes increased Fatty Acid import into Lipid Droplets

(A) SG formation timeline during starvation showing LD accumulation. HEK293T cells expressing CRISPR/Cas9 tagged PABPC1-DDR2were starved in a fuel and serum depleted media containing FA-free BSA for indicated amounts of time, FA dye (Bodipy-C12, $1 \mu \mathrm{M}$ ) was added 30 minutes prior to the imaging together with Hoechst $(10 \mu \mathrm{g} / \mathrm{ml})$. Arrows indicate SGs, Scale bar $5 \mu \mathrm{m}$.

(B) SG formation timeline during sodium arsenite treatment showing LD accumulation. Cells expressing CRISPR/Cas9 tagged PABPC1-DDR2 were incubated with arsenite $(100 \mu \mathrm{M})$ for indicated amounts of time, FA dye (Bodipy-C12, $1 \mu \mathrm{M}$ ) was added 30 minutes prior to the imaging together with Hoechst $(10 \mu \mathrm{g} / \mathrm{ml})$. Arrows indicate SGs, Scale bar $5 \mu \mathrm{m}$.

(C) Quantification of SG formation, FAO, and LD accumulation during fuel starvation (corresponding to Figure 2A). Graph shows \% of cells with SGs in the population and LD fluorescence intensity, mean \pm SD. Pearson Correlation coefficient ( $r$ ) is 0.98 . Fold change in FAO is overlaid, refer to the Figure $1 \mathrm{~K}$ for quantification.

(D) Quantification of SG formation and LD accumulation during arsenite treatment (Corresponding to Figure 2B). Graph shows \% of cells with SGs in the population and LD fluorescence intensity, mean \pm SD. Pearson Correlation coefficient (r) is 0.99 .

(E) Disruption of SG formation results in the decrease in FA accumulation. Cells expressing CRISPR/Cas9 tagged PABPC1-DDR2 were incubated with arsenite $(100 \mu \mathrm{M})$ or arsenite and cycloheximide $(10 \mu \mathrm{g} / \mathrm{ml})$ for 90 minutes. FA dye (Bodipy-C12, $1 \mu \mathrm{M})$ was added 30 minutes prior to the imaging. Representative confocal planes are shown, scale bar $5 \mu \mathrm{m}$.

(F) Quantification of FA (Bodipy-C12) accumulation in LDs (H). Cells expressing CRISPR/Cas9 tagged PABPC1-DDR2 were incubated with arsenite $(100 \mu \mathrm{M})$ or arsenite and cycloheximide $(10 \mu \mathrm{g} / \mathrm{ml})$ for $90 \mathrm{~min}$. FA dye (Bodipy-C12, Red, $1 \mu \mathrm{M}$ ) was added 30 minutes prior to the 
experiment. Graph represents fluorescence intensity of bodipy tagged FAs in LDs, mean \pm SEM, $\mathrm{n}=30$.

(G) Schematic of lipid (FAs and Cholesterol) import into LDs

(H) Quantification showing increase in accumulation of FAs but not cholesterol in LDs during SG formation (SGs were formed by incubation for 60 minutes with arsenite $100 \mu \mathrm{M}$ ). Graphs show mean $\pm \operatorname{SEM}, \mathrm{n}=30$.

(I) Intensity profile of FAs accumulation in the LDs. Cells were incubated with arsenite for 1h, Bodipy (Green, $1 \mu \mathrm{M}$ ) was added 30 minutes before the imaging to stain the LDs. FA (Bodipy$\mathrm{C} 12$, Red, $1 \mu \mathrm{M})$ was added at the start of the acquisition. Confocal images were taken every minute for 15 minuntes, intensity profiles of the images are shown, scale bar $1 \mu \mathrm{m}$ (refer to Figure S3A).

(J) Intensity profile of cholesterol accumulation in the LDs. Cells were incubated with arsenite $(100 \mu \mathrm{M})$ for $1 \mathrm{~h}$, Bodipy-C12 (Red, $1 \mu \mathrm{M})$ was added 30 minutes before the imaging to stain the LDs. Cholesterol (Bodipy-cholesterol, Green, $1 \mu \mathrm{M}$ ) was added at the start of the acquisition. Confocal images were taken every minute for 15 minutes, intensity profiles of the images are shown, scale bar $1 \mu \mathrm{m}$ (refer to Figure S3B).

(K) Quantification of TAG accumulation (left) and glycerol release (right) during starvation. HEK293T cells were starved for 4 or 18 hours. Graphs show mean \pm SEM.

(L) Analysis of FAO during short-term and long-term starvation comparing to control conditions. SH-SY5Y cells with or without TDP43 Q331K-GFP mutation (refer to Figure S2A-C) were starved for 18 hours with or without FA import inhibition with etomoxir (20 $\mu \mathrm{M})$, added 1 hour prior to the start of the measurement, and analyzed using Seahorse Mito Fuel Flex test. Line graph represents fatty acid dependency mean \pm SEM.

(M) Schematic showing FA trafficking in short- and long-term starvation.

Figure 3. Stress Granules interactome reveals mitochondrial and Lipid Droplet association

(A) SG-organelle proximity showing confocal images of LDs (Bodipy-C12), Mitochondria (Mito Red), Peroxisomes (mCH-SKL), nucleus (Hoechst), Lysosome (Lamp1-mCH), Endoplasmic 
reticulum (CALR21-mCH-KDEL), and Golgi apparatus (B4GALT182-mCH) adjacent to SGs (PABPC1-DDR2). HEK293T cells expressing CRISPR/Cas9 tagged PABPC1-DDR2 were starved for 9 hours in a fuel and serum depleted media containing FA free BSA, organelles were visualized with live cell dyes (added 30 minutes prior to the imaging) or plasmid encoded markers. Images show representative confocal planes, scale bar $1 \mu \mathrm{m}$ (refer to Figure S4A).

(B) Quantification of SG proximity to membrane bound organelles showing absolute SG proximity compared to proximity of randomly generated SG sample (refer to Figure S4B). Graphs show a ratio of proximal SGs in a sample, mean \pm SEM, $n=30$.

(C) Tripartite association between SG (PABPC1-DDR2), LD (Bodipy-C12), and Mitochondria (Rhodamine800). Cells expressing CRISPR/Cas9 tagged PABPC1-DDR2 were starved for 9 hours, organelles were visualized with live cell dyes (added 30 minutes prior to the imaging). Kymograph was recorded with 2 second interval between frames for $5 \mathrm{~min}$, scale bar $1 \mu \mathrm{m}$.

(D) Schematic of protein identification by Mass Spectrometry. HEK293T cells expressing PABPC1 fused to promiscuous biotin ligase BirA (R118G) and GFP were subjected to arsenite $(100 \mu \mathrm{M}), 2$-bromopalmitate (2-BP, $200 \mu \mathrm{M})$, or fuel starvation for 9 hours with addition of biotin for 6 hours. Cells were lysed and biotinylated proteins were captured on magnetic beads, followed by on bead digestion and LC/MS/MS (refer to Figure S4C-F).

(E) Gene ontology (GO) analysis of SG enriched proteins grouped by conditions. Shown annotations are significantly enriched and hierarchically arranged. Analysis is performed by STRING web tool (https://string-db.org/). Circle diagram showing PABPC1 interactome identified in all conditions, 15 proteins are present in all SG conditions but not in the control conditions.

(F) Network of proteins that are present in all SG conditions, but not in the control conditions. Mitochondrial membrane protein is indicated in red.

(G) Analysis of SG enriched fraction by comparison to G3BP interacting proteins (Isabelle et al., 2012), refer to Figure S4G for comparison with interactomes of FUS, TDP43, and SG proteomics. Protein interaction networks and Venn diagrams are shown. Mitochondrial membrane protein VDAC2 is indicated in red. 
(H) Bimolecular Fluorescence complementation experiment showing interaction of SGs with VDAC2. CRISPR/Cas9 tagged PABPC1-mCH-VenusC (refer to Figure S4H-K) were transfected with VenusN, VenusN-VDAC2, or PABPC1-VenusN plasmids. SGs were formed with arsenite $(100 \mu \mathrm{M})$. The change in Venus fluorescence intensity in the SG area was recorded by comparing cells after SG formation (1 hour of arsenite treatment) and 3 hours after SG formation. Fold change of fluorescent intensity is shown comparing to control, mean $\pm \operatorname{SEM}, \mathrm{p}<0.05, \mathrm{n}=30$.

(I) Immunoprecipitation of VDAC2 in control and long-term starvation conditions.

(J) Protein interaction network of proteins shared with LD proteomics (Roesch et al, 2016). Mitochondrial membrane proteins are indicated in red. Venn diagram shows fraction of shared proteins.

(K) Alignment of VDAC proteins from S. cerevisiae (Por1 and Por2) and H. sapiens (VDAC1,2,3). Refer to Figure S5A for full alignment. Alignment was performed using ClustalW and Jalview2 tools (Waterhouse et al., 2009; Sievers et al.,.2011).

Figure 4. VDAC undergoes reversible clustering during Stress Granule formation

(A) Yeast S. cerevisiae VDAC, Por1, clusters during starvation. Yeast cells expressing POR1-GFP endogenous tag were grown to middle log phase and starved for 1 hour in glucose free media. Mitochondria were stained with Mito Red dye $(1 \mu \mathrm{M})$ for 30 minutes prior to the imaging. Live cells were seeded on Concanavalin A coated plates and visualized. Representative confocal frames are shown, scale bar $1 \mu \mathrm{m}$. Arrow heads indicate VDAC inclusions.

(B) Yeast VDAC clustering coincides with its depletion from mitochondrial membrane. Quantification of the fluorescent intensity of VDAC on the mitochondrial membrane is shown in blue (inclusions were not counted in the quantification), mean \pm SEM, $n=30$. Quantification of the ratio of cells with VDAC inclusions in the population is shown in red, mean $\pm \mathrm{SEM}, \mathrm{n}=100$, $*$ indicates $\mathrm{p}<0.05$.

(C) Yeast Stress Granules colocalize with Por1 inclusions. Yeast cells expressing endogenously tagged POR1-mCH (VDAC) and PAB1-GFP (PABPC1 orthologue) were grown to middle log phase and starved in glucose free media for 1 hour prior to the imaging. Representative confocal planes are shown, scale bar $1 \mu \mathrm{m}$. Arrowheads indicate Stress Granules. 
(D) Yeast VDAC undergoes reversible clustering. Yeast cells expressing POR1-GFP endogenous tag were grown to middle log phase and starved for 1 hour in glucose free media while confocal imaging. Mitochondria were stained with Mito Red dye $(1 \mu \mathrm{M})$ for 30 minutes prior to the imaging. Live cells were seeded on Concanavalin A coated plates and visualized. Representative confocal planes of the mitochondria are shown, scale bar $1 \mu \mathrm{m}$. Recovery was performed on the microscope by addition of glucose containing media and visualizing cells every 5 minutes for 30 minutes.

(E) Western blot of Por1-GFP in control and starvation conditions. Yeast were grown to mid-log phase and glucose starved for 1 hour prior to lysis.

(F) VDAC2 clustering in mammalian cells during SGs formation. HEK293T cells overexpressing GFP-VDAC2 were grown in control, arsenite $100 \mu \mathrm{M}$, arsenite and cycloheximide $(10 \mu \mathrm{g} / \mathrm{ml})$, and starvation in PBS. Quantification shows the ratio of cells with VDAC inclusions in the population, mean \pm SEM, $*$ - $p<0.05$.

(G) Western blot showing VDAC2 distribution in soluble and insoluble fractions, quantification shows the ratio of solubleVDAC2 to insoluble VDAC2 normalized to SDHA levels.

(H) Clustering of endogenous VDAC2 during long term starvation in human cells. SHSY5Y cells were starved for 18 hours. Quantification the ratio of cells containing aggregates in the population, mean \pm SEM.

Figure 5. VDAC2 is essential for mitochondrial influx of fatty acids

(A) FAs accumulate in VDAC2 knockout. Control and VDAC2 knockout cells (Clone 2) (refer to Figure S6A) were grown in normal or starvation media for 4 hours. FA dye (Bodipy-C12, 1 $\mu \mathrm{M}$ ) was added 30 minutes prior to the imaging together with Hoechst $(10 \mu \mathrm{g} / \mathrm{ml})$. Representative images are shown, Scale bar $5 \mu \mathrm{m}$. Fold change of lipid droplet accumulation is indicated in the upper right corner, $\mathrm{p}<0.05, \mathrm{n}=30$.

(B) Intensity profile of FAs accumulation in the LDs. HEK293T cells were incubated with Bodipy (Green, $1 \mu \mathrm{M}$ ) 30 minutes before the imaging to stain the LDs. FA (Bodipy-C12, Red, $1 \mu \mathrm{M}$ ) was added at the start of the acquisition. Confocal images were taken every minute for 15 minutes, intensity profiles of the images are shown, scale bar $1 \mu \mathrm{m}$. Quantification showing increase in accumulation of FAs in LDs of VDAC2 knockout cells (Clone 7). Graphs show mean \pm SEM. 
(C) Analysis of Lipid Droplet - Mitochondria contacts in WT and VDAC2 KO cells. Representative 3D reconstructions from confocal images are shown. Quantification shows ratio of lipid droplets proximal to mitochondria mean $\pm \mathrm{SEM}$.

(D-E) Analysis of FAO during short term starvation (E), and long-term starvation (D) in control and VDAC2 KO cells. Control, VDAC2 knockout cells, and HADHA knockout cells were starved for 4 hours to induce FAO response and analyzed using Seahorse Mito Fuel Flex test. Etomoxir was used to inhibit FA import into mitochondria. Graph represents OCR used for FAO (E) and fold change in FAO (D), mean $\pm \mathrm{SEM}$.

(F-G) GC-FID analysis of FA content in purified mitochondria in control and VDAC2 KO cells. HEK293T cells were starved with or without FA import inhibition prior to mitochondria extraction. Purified mitochondrial FA profiles were obtained using GC-FID. Graphs show saturated FA (sFA) profiles in all conditions (refer to Supplementary Figure S6G) (G), increase in all sFA (H, left panel), and decrease in sFA in FA import inhibition (H, right panel), mean $\pm \mathrm{SEM}$.

I) Western blot of VDAC2 in liposomes (refer to Supplementary Figure S6H).

(J) Confocal microscopy of liposomes with labeled VDAC2-Cy3.

(K) Retention of FA in VDAC2 liposomes. Graph shows fold change of free fatty acids, quantified in liposomes with and without VDAC2, treated and not treated with FAs, mean $\pm \mathrm{SEM}$.

(L) Confocal images of VDAC2-Cy3 liposomes before and after addition of pure SG fraction (refer to Supplementary Figure S6J). Graph show ratio of aggregates of VDAC2, mean \pm SEM.

Figure 6. Stress Granule mediated signaling regulates VDAC clustering and fatty acid oxidation

(A) Schematic of mTOR and GSK3 regulation during SG formation. During normal conditions mTOR phosphorylates 4EBP allowing translation initiation. Downregulation of mTOR during low nutrient conditions results in translation inhibition and SG formation, in turn SG formation results in mTOR inhibition and GSK3 activation. GSK3 phosphorylates VDAC2. 
(B) Long term starvation leads to mTOR inactivation. Cells were incubated in fuel starvation media for indicated amounts of time. mTOR activity was assessed by 4EBP phosphorylated (Thr46) state. Ratio of phosphorylated to non-phosphorylated 4EBP is shown.

(C) mTOR localized to SGs during starvation. Cells expressing PABPC1-DDR2 were grown in control conditions and starved for 9 hours. Cells were fixed with 4\%PFA and mTOR was visualized with an antibody. Representative confocal frames are shown, scale bar $1 \mu \mathrm{m}$.

(D) Long term starvation leads to GSK3 activation. HEK293T cells were incubated in fuel starvation media for indicated amounts of time or with mTOR inhibitor for 1 hour (SMI-4a, 50 $\mu \mathrm{M})$. GSK3 alpha activity was assessed by its phosphorylation state. Ratio of phosphorylated to non-phosphorylated GSK3 is shown.

(E) Phosphorylation of VDAC2 clusters detected by anti-phospho Serine antibody. HEK293T cells expressing GFP-VDAC2 were grown in control conditions and treated with arsenite $(100 \mu \mathrm{M})$ for 1 hour. Representative confocal images of VDAC2 clusters are shown, scale bar $1 \mu \mathrm{m}$.

(F) SG formation and VDAC2 clustering is regulated by GSK3 kinase. Cells expressing endogenously tagged PABPC1-DDR2 or overexpressing GFP-VDAC2 were incubated with arsenite $(100 \mu \mathrm{M})$ or with arsenite in the presence of GSK3 inhibitors (BIO, SB-415286, 100 $\mu \mathrm{M})$. Confocal images are shown, scale bar $5 \mu \mathrm{m}$.

(G) SG formation and VDAC2 clustering is regulated by GSK3 kinase. Cells over expressing GFP-VDAC2 were incubated with arsenite $(100 \mu \mathrm{M})$ or with arsenite in the presence of GSK3 inhibitors (BIO, SB-415286, 100 $\mu \mathrm{M})$. SG formation was quantified as a number of cells with inclusions in the population. Graph shows ratio of cells with SGs, mean \pm SEM.

(H) Analysis of FAO in short-term starvation during Stress Granule formation. HEK293T were starved for 2 hours, then treated for 1 hour with 1-NA-PP1, SMI-4a, AS-703026, ruxolitinib, KN93, TGX-221, AG-17 (100 $\mu \mathrm{M}$ each) and FAO was analyzed using Seahorse Mito Fuel Flex test. Graph represents reliance on FA to maintain respiration of starved/control conditions, mean \pm SEM. Negative control was grown in normal non starvation media. Refer to figure S7I for SG formation data. 
(I) Analysis of FA contribution to OCR during long-term starvation during GSK3 inhibition. Cells were starved for 18 hours with or without GSK3 inhibitors (BIO, SB-415286, 20 $\mu \mathrm{M}$ ) and analyzed using Seahorse Mito Fuel Flex test. Graph represents fold change in reliance on FA to maintain respiration of starved/control conditions, mean \pm SEM

(J) Model of SG regulation of VDAC2 clustering and FA trafficking during starvation. During short term starvation FAs are imported into mitochondria for FAO, mTOR is active and blocks GSK3 activity. During long term starvation SG formation leads to inhibition of mTOR, which in turn leads to the activation of GSK3 and phosphorylation of VDAC2. Phosphorylated VDAC2 undergoes clustering which results in downregulation of FAO and redirecting of FA to LDs.

Figure 7 Patient derived iPSC differentiated to neural precursors exhibit delayed FAO inhibition response

(A) Schematic of human iPSC differentiation. Refer to Figure S8A.

(B) Western blot of stem cell and neuronal lineage markers. Quantification shows fold change relative to GAPDH. Refer to Figure S8B.

(C-D) Confocal microscopy of human iPSC with and without TDP43 mutation in control and starvation conditions. Cells were starved for 4 hours prior to fixation. SG markers, G3BP and TDP43, were visualized with antibodies. Quantification shows ratio of cells with SGs in the population at 4 hours of starvation, mean \pm SEM.

(E) Confocal microscopy of FA accumulation during starvation. Human iPSC differentiated to Neuronal progenitors were starved in a fuel and serum depleted media containing FA-free BSA for indicated amounts of time, FA dye (Bodipy-C12, $1 \mu \mathrm{M}$ ) was added 30 minutes prior to the imaging together with Hoechst $(10 \mu \mathrm{g} / \mathrm{ml})$.

(F) Analysis of FAO during starvation. Human iPSC differentiated to neuronal progenitor cells with or without TDP43 mutation (refer to Supplementary Figure S8D) were starved for 2, 4, and 6 hours and analyzed using Seahorse Mito Fuel Flex test. Line graph represents fatty acid dependency mean $\pm \mathrm{SEM}, *$ indicates $\mathrm{p}<0.05$.

\section{Figure S1.}


(A) Visualization of cells in the conditions used for fatty acid dependency experiment. HEK293T cells were starved in no glucose, no glutamine, or no glucose and no glutamine lipid free media for 3 hours. Then cells were visualized by confocal microscopy, scale bar $5 \mu \mathrm{m}$.

(B) HADHA knockout confirmation

(C) Schematic of CRISPR/Cas9 tagging of HADHA with GFP showing regions used for homologous recombination and verified sequence region after integration.

(D) HADHA-GFP localizes to mitochondria. HEK293T HADHA-GFP cells were stained with MitoRed $(1 \mu \mathrm{M})$ for 30 minutes. Confocal images are shown, scale bar $5 \mu \mathrm{m}$.

(E) Confirmation of HADHA endogenous tagging. Immunofluorescence showing co-localization of HADHA-GFP with HADHA antibodies, confocal images are shown, scale bar $5 \mu \mathrm{m}$.

(F) HADHA levels increase during starvation. CRISPR/Cas9 tagged HADHA-GFP cells were starved for 6 hours or treated with FAO activators - clofibrate, rosiglitazone, and GW501516 (100 $\mu \mathrm{M}$ each) for 3 hours, fold increase in HADHA-GFP fluorescence intensity is indicated in the upper right corner of the confocal planes, mean $\pm \mathrm{SEM}, \mathrm{p}<0.05$

(G) Schematic of CRISPR/Cas9 tagging of PABPC1 showing regions used for homologous recombination and verified sequence region after integration.

(H) SG formation timeline of PABPC1-DDR2. CRISPR/Cas9 tagged PABPC1-DDR2 HEK293T cells were incubated with arsenite $(200 \mu \mathrm{M})$ for indicated amounts of time. Confocal planes are shown, arrows indicate SGs, scale bar $1 \mu \mathrm{m}$.

(I) Western blot showing control and CRISPR/Cas9 PABPC1-DDR2 samples. Anti-DDR2 antibodies are used.

(J) Immunofluorescence showing SG markers localization to the inclusions. PABPC1-DDR2 cells were starved for 9 hours or treated with arsenite $(100 \mu \mathrm{M})$ for 1hour. Cells were fixed in paraformaldehyde (PFA) and stained with anti G3BP, and anti TIA1 antibodies. Confocal planes are shown, inlets show SGs, number in the inlet indicates co-localization coefficient of PABPC1 and G3BP, Scale bar $5 \mu \mathrm{m}$. 
(K) SG formation timeline of PABPC1-DDR2. CRISPR/Cas9 tagged PABPC1-DDR2 cells grown to $90 \%$ confluency were incubated in complete media without changing for indicated amount of time. Recovery indicate media replacement after 4 days of incubation. Representative confocal planes are shown, arrows indicate SGs, scale bar $5 \mu \mathrm{m}$.

(L) SG formation timeline of PABPC1-DDR2. CRISPR/Cas9 tagged PABPC1-DDR2 cells were incubated in phosphate buffered saline (PBS) for indicated amount of time. Representative confocal planes are shown, arrows indicate SGs, scale bar $5 \mu \mathrm{m}$.

(M) Quantification of cells with SGs (\%) in starvation and arsenite conditions, mean \pm SEM, $\mathrm{n}=$ 100 .

(N) Schematic of CRISPR/Cas9 tagging of PABPC1with $\mathrm{mCH}$ showing regions used for homologous recombination and verified sequence region after integration.

(O) Western blot showing PABPC1-mCH clones

(P) Immunofluorescence showing SG marker localization to the PABPC1-mCH inclusions. PABPC1-mCH cells were treated with arsenite $(100 \mu \mathrm{M})$ for 1hour. Cells were fixed in PFA and stained with anti TIA1 antibodies. Hoechst $(10 \mu \mathrm{g} / \mathrm{ml})$ was used to stain the nucleus 30 minutes prior to imaging. Confocal planes are shown, scale bar $5 \mu \mathrm{m}$.

(Q) Nonsignificant changes in mitochondrial potential during short term starvation. Cells were grown in control or starvation media for 6 hours. 30 minutes CCCP $(4 \mu \mathrm{M})$ incubation was used as a negative control. Representative confocal planes are shown, scale bar $5 \mu \mathrm{m}$. Graph shows average mitochondrial fluorescence intensity in starvation and control conditions, mean \pm SEM.

(R) Nonsignificant changes in oxidative stress during short term starvation. Cells were grown in control or starvation media for 6 hours. H2-DFCDA $(100 \mu \mathrm{M})$ was added 1 hour prior to the imaging. Hoechst $(10 \mu \mathrm{g} / \mathrm{ml})$ was used to stain the nucleus 30 minutes prior to imaging. Representative confocal planes are shown, scale bar $5 \mu \mathrm{m}$. Graph shows average cytoplasmic fluorescence intensity in starvation and control conditions, mean \pm SEM.

\section{Figure S2}

(A) Schematic of CRISPR/Cas9 modification of TARDBP region showing endogenous tagging with GFP and introduction of Q331K mutation. 
(B) Western blot confirmation of CRISPR/Cas9 TDP43-Q331K-GFP and control SHSY5Y cells.

(C) Colocalization of TDP43-Q331K-GFP with TDP43 antibody in the nucleus and nuclear inclusions. CRISPR/Cas9 TDP43-Q331K-GFP SH-SY5Y cells were fixed in PFA and stained with anti-TDP43 antibody $(\mathrm{ab})$. Hoechst $(10 \mu \mathrm{g} / \mathrm{ml})$ was added 15 min prior to the imaging. Representative confocal planes are shown, scale bar $5 \mu \mathrm{m}$. Inlet demonstrates nuclear inclusions. Intensity profile shows fluorescence intensity of TDP43-Q331K-GFP (green) and antibodydetected TDP43 (red) across nuclear inclusions.

(D) SG formation in SH-SY5Y cells with arsenite and 2-BP. SH-SY5Y cells were treated with vehicle, arsenite $(100 \mu \mathrm{M})$, or 2-BP $(200 \mu \mathrm{M})$ for 1 hour, then fixed with PFA. SG markers were visualized with anti-TIA1 and anti-G3BP antibodies. Hoechst $(10 \mu \mathrm{g} / \mathrm{ml})$ was added 15 min prior to the imaging. Representative confocal planes are shown, scale bar $5 \mu \mathrm{m}$. Arrowheads indicate SGs.

(E) SG formation in cells with or without TDP43 Q331K mutation during starvation. SHSY5Y cells were incubated in fuel starvation media for indicated amounts of time, then fixed in PFA and stained with G3BP antibody. Representative confocal images are shown, scale bar $5 \mu \mathrm{m}$. Hoechst $(10 \mu \mathrm{g} / \mathrm{ml})$ was used to stain the nucleus. Number in the upper left corner of merged image represents \% of cells with SGs in the population. Arrowheads indicate SGs

(F) Inhibition of fatty acid import to mitochondria decreases oxidative stress during starvation in TDP43 Q331K mutant cells. Cells with TDP43 Q331K mutation were incubated in starvation media with vehicle or etomoxir $(5 \mu \mathrm{M})$. H2-DFCDA $(100 \mu \mathrm{M})$ was added 1 hour prior to the imaging, scale bar $5 \mu \mathrm{m}$

(G) Confocal microscopy of SG markers in SHSY5Y cells treated with arsenite. Intensity profile of SG is shown.

\section{Figure S3.}

(A) FAs accumulation in the LDs. Cells were incubated with arsenite for 1 hour, Bodipy (Green, $1 \mu \mathrm{M}$ ) was added 30 minutes before the imaging to stain the LDs. FAs (Bodipy-C12, Red, $1 \mu \mathrm{M}$ ) were added at the start of the acquisition. Confocal images were taken every minute for 15 minutes, scale bar $1 \mu \mathrm{m}$ 
(B) Confocal microscopy of HADHA during long-term starvation. HEK293T cells expressing endogenously tagged PABPC1-DDR2 were starved for 15 hours. Quantification of HADHA fluorescence intensity in mitochondria of cells with and without SGs is shown, mean \pm SEM, $n=25$.

(C) SG formation and recovery showing disruption of SG formation during arsenite and cycloheximide treatment. Cells expressing PABPC1-DDR2 were incubated with arsenite $(100 \mu \mathrm{M})$, cycloheximide $(10 \mu \mathrm{g} / \mathrm{ml})$, or both for $90 \mathrm{~min}$. Representative confocal planes are shown, scale bar $1 \mu \mathrm{m}$. Recovery indicate replacement the media with a complete medium for 1 hour.

(D) SG formation leads to lipid accumulation. Graph shows total lipid quantification during arsenite $(100 \mu \mathrm{M})$, or arsenite and cycloheximide $(10 \mu \mathrm{g} / \mathrm{ml})$ treatment, mean $\pm \mathrm{SEM}, \mathrm{n}=3$.

(E) Free FA quantification showing non-significant changes during arsenite $(100 \mu \mathrm{M})$, or arsenite and cycloheximide $(10 \mu \mathrm{g} / \mathrm{ml})$ treatment, Lipid mix 1 (Sigma) containing $2 \mu \mathrm{g} / \mathrm{ml}$ arachidonic, $10 \mu \mathrm{g} / \mathrm{ml}$ linoleic, linolenic, myristic, oleic, palmitic and stearic, and $0.22 \mathrm{mg} / \mathrm{ml}$ cholesterol is used as a calibration, mean \pm SEM, $\mathrm{n}=3$.

(F) TDP43 overexpression leads to FA accumulation. Cells expressing PABPC1-DDR2 were transfected with IFP or TDP43-IFP plasmids and grown in normal conditions. FA dye (BodipyC12) were added to visualize LD 1 hour prior to imaging. Representative confocal images are shown. Quantification shows fluorescence intensity in LDs, mean \pm SEM.

(G) Overview of FA trafficking during starvation. During short-term starvation cells upregulate FA import into mitochondria and upregulate FAO. During long-term starvation cells import FA into LDs and downregulate FAO.

\section{Figure S4}

(A) SG-organelle proximity showing confocal images of LDs (Bodipy-C12), Mitochondria (Mito Red), Peroxisomes (mCHskl), nucleus (Hoechst), Lysosome (Lamp1-mCH), Endoplasmic reticulum (CALR21-mCHkdel), and Golgi apparatus (B4GALT182-mCH) in PABPC1-DDR2 cells starved for $9 \mathrm{~h}$ in a fuel and serum depleted media containing FA free BSA. Images show confocal 2D planes, scale bar $1 \mu \mathrm{m}$.

(B) Comparison of SG proximity to LDs with randomly generated SG sample proximity. Image showing LDs (D) was merged with randomly generated SGs, arrows indicate SGs proximal to LDs 
(C) Stress Granule formation visualization with PABPC1-BirA (R118G (*))-GFP. Cells expressing PABPC1-BirA(R118G)-GFP were treated with arsenite $(100 \mu \mathrm{M})$ for 1 hour. Confocal images are shown, scale bar $1 \mu \mathrm{m}$.

(D) Increase in protein biotinylation after addition of biotin to the media. Cells expressing PABPC1-BirA(R118G)-GFP were treated with to arsenite $(100 \mu \mathrm{M})$, or fuel starvation for 9 hours with addition of biotin for 6 hours. Cells were lysed and biotinylated proteins were captured on magnetic beads, followed by western blot. Biotinylated proteins were visualized by streptavidinHRP detection.

(E-F) BioID identifies proteins inside SG compartment, as well as SG interacting proteins. Cells expressing PABPC1-BirA(R118G)-GFP were treated with arsenite(100 $\mu \mathrm{M})$ for 1 hour, vehicle or biotin $(100 \mu \mathrm{M})$ were added to the media for 4 hours. Cells were fixed in PFA and stained with streptavidin-Cy3. Hoechst $(10 \mu \mathrm{g} / \mathrm{ml})$ was used to stain the nucleus 30 minutes prior to imaging. Confocal planes are shown, scale bar $1 \mu \mathrm{m}$. Graphs show fluorescence intensity of streptavidinCy3 (biotinylated proteins) and PABPC1-BirA(R118G)-GFP (Stress Granule) across SG inclusion.

(G) Analysis of SG enriched fraction by comparison to other studies of SG interacting proteins. PABPC1 interactors in SG were compared to FUS interactome (Kamelgran et al., 2016), TDP43 interactome (Freibaum et al., 2010), and SG proteomics (Jain et al., 2012). Protein interaction networks and Venn diagrams are shown. Mitochondrial membrane proteins are indicated in red.

(H) Schematic of CRISPR/Cas9 tagging of PABPC1 with $\mathrm{mCH}-\mathrm{VenusC}(\mathrm{Vc})$ showing regions used for homologous recombination and verified sequence region after integration.

(I) Western blot showing PABPC1-mCH-Vc clone.

(J) Immunofluorescence showing SG marker localization to the PABPC1-mCH-Vc inclusions. PABPC1-mCH-Vc cells were treated with arsenite $(100 \mu \mathrm{M})$ for 1 hour. Cells were fixed in PFA and stained with anti TIA1 antibodies. Hoechst $(10 \mu \mathrm{g} / \mathrm{ml})$ was used to stain the nucleus 15 minutes prior to imaging. Confocal planes are shown, scale bar $5 \mu \mathrm{m}$.

(K) Bimolecular Fluorescence complementation experiment showing interaction of SGs with VDAC2. CRISPR/Cas9 tagged PABPC1-mCH-VenusC were transfected with VenusN, VenusN- 
VDAC2, or PABPC1-VenusN plasmids. SGs were formed with arsenite $(100 \mu \mathrm{M})$. The change in Venus fluorescence intensity in the SG area was recorded by comparing cells after SG formation (1-hour arsenite treatment) and 3 hours after SG formation. Confocal images are shown, scale bar $5 \mu \mathrm{m}$. Fold change of fluorescent intensity is shown in the upper left corner of the 3 hours planes, comparing to control, mean $\pm \mathrm{SEM}, \mathrm{p}<0.05, \mathrm{n}=30$.

\section{Figure S5}

(A) Alignment of VDAC proteins from S. cerevisiae (Por1 and Por2) and H. sapiens (VDAC1,2,3). Alignment was performed using ClustalW and Jalview2 tools (Waterhouse et al., 2009; Sievers et al., 2011).

(B) Average distance tree for VDAC proteins from S. cerevisiae (Por1 and Por2) and H. sapiens (VDAC1,2,3). The tree was constructed using BLOSUM62, Jalview2 tool (Waterhouse et al., 2009)

(C) Association between SG (Pab1-GFP) and Mitochondria (MitoRed) in yeast. Yeast cells were grown to mid log phase and starved in no glucose media for 1 hour, organelles were visualized with live cell dye (added 30 minutes prior to the imaging). Kymograph was recorded with 2 second interval between frames for $5 \mathrm{~min}$, scale bar $1 \mu \mathrm{m}$.

(D) Immunofluorescence showing VDAC2 cluster proximity to SGs. HEK293T cells expressing GFP-VDAC2 were treated with arsenite $(100 \mu \mathrm{M})$ for an hour and fixed with PFA. VDAC2 expression was confirmed with VDAC2 antibody, SGs were visualized with G3BP antibody. Confocal planes are shown, scale bar $5 \mu \mathrm{m}$.

(E) VDAC2 clustering in mammalian cells during SGs formation. Cells overexpressing GFPVDAC2 were visualized in control, arsenite $100 \mu \mathrm{M}$, arsenite and cycloheximide $(10 \mu \mathrm{g} / \mathrm{ml})$, and starvation in PBS. Cells were imaged every 10 minutes for 1 hour. Representative confocal planes are shown, scale bar $1 \mu \mathrm{m}$.

(F) VDAC2 quantification on the mitochondrial membrane during SG formation. Cells were grown to $90 \%$ confluency and starved for 3 hours, followed by arsenite or arsenite and cycloheximide treatment for 1 hour and whole lysate extraction or mitochondria purification. 
Western blot quantification shows the ratio of mitochondrial VDAC2 to overall VDAC2 obtained from cell lysis normalized to TOM22 levels.

(G) VDAC2 clustering depends on Ser112 (Ser122 in VDAC2 with N terminal tail). Conserved S residues were mutated to A or D and GFP-VDAC2 clustering during SG formation was visualized. Quantification shows ratio of cells with VDAC2 inclusions in the population. Quantification shows ratio of cells with VDAC2 inclusions in the population, mean $\pm \mathrm{SEM}$.

(H) Confocal microscopy of SG formation during long term starvation in WT, VDAC2 KO, and HADHA KO cells. HEK293T cells were starved for 18hours. SG were visualized with G3BP antibody. Quantification represents ratio of cells with $\mathrm{SGs}$ in the population, mean $\pm \mathrm{SEM}$.

\section{Figure S6}

(A) Confirmation of CRISPR/Cas9 knockout (KO) of VDAC2. Western blot showing VDAC2 and not VDAC1 downregulation. Schematic of VDAC2 knockout showing sequenced region and disruption of the start of the open reading frame.

(B) FA import into Mitochondria in control and VDAC2 KO (Clone2). Control and VDAC2 KO cells were stained by Rhodamine $800(1 \mu \mathrm{M})$ and Bodipy-C12, Red $(1 \mu \mathrm{M})$ for $30 \mathrm{~min}$, fatty acid (Bodipy-C12, red) import to mitochondria was quantified as an increase in the fluorescence intensity in the mitochondria. Quantification showing increase in FA accumulation in mitochondria in control but not in $\operatorname{VDAC} 2 \mathrm{KO}$. Graphs show mean $\pm \mathrm{SEM}, \mathrm{n}=30$. Intensity profiles of representative confocal images are shown, scale bar $1 \mu \mathrm{m}$.

(C-D) VDAC2 is proximal to LDs. Cells were fixed in 4\%PFA. Mitochondria was visualized with Tom 22 antibody, LDs were stained with Bodipy (Green, $1 \mu \mathrm{M})$, representative confocal image is shown, scale bar $1 \mu \mathrm{m}$.Quantification (D) shows ratio of organelles with VDAC2 association, determined by the overlap in fluorescence intensity, mean \pm SEM.

(E) VDAC2 complementation reduces lipid content of VDAC2 knockout cells in short-term starvation. VDAC2 knockout cells (Clone 2) were transfected with GFP-VDAC2, GFPVDAC2S112D plasmid or GFP control. LD dye (Bodipy-C12, $1 \mu \mathrm{M}$ ) was added 30 minutes prior to the imaging together with Hoechst $(10 \mu \mathrm{g} / \mathrm{ml})$, cells were starved for 3 hours prior to the imaging. 
Representative images are shown. Fold reduction of lipid accumulation in GFP-VDAC2 expressing cells is shown in the upper left corner, $\mathrm{p}<0.05, \mathrm{n}=30$.

(F) LDs accumulate in clusters. PABPC1-DDR2 cells were treated with arsenite $(100 \mu \mathrm{M})$ for 1 hour, FAs (Bodipy-C12, Red, $1 \mu \mathrm{M}$ ) were added 1 hour before the acquisition to stain the LDs. Confocal images are shown, scale bar $5 \mu \mathrm{m}$. Inlets indicate single lipid droplets in control conditions, and a cluster of lipid droplets in arsenite conditions.

(G) GC-FID analysis. Graphs show sFA content in control and VDAC2 KO purified mitochondria in control, short term starvation, and FA import inhibition conditions.

(H) Western blot of VDAC2 incorporation into liposomes. Quantification shows ratio of VDAC2 pelleted with liposomes.

(I) Confocal microscopy of liposomes before and after extrusion.

(J) Confocal microscopy of isolated SGs, scale bar $1 \mu \mathrm{m}$.

\section{Figure S7}

(A) Immunofluorescence showing phospho-4EBP downregulation in starvation conditions which coincides with SG formation. PABPC1-DDR2 cells were starved for 9 hours. Cells were fixed in PFA and stained with anti-phosphoThr46-4EBP antibody. Hoechst $(10 \mu \mathrm{g} / \mathrm{ml})$ was used to stain the nucleus. Confocal planes are shown, scale bar $5 \mu \mathrm{m}$. Arrowheads indicate SGs.

(B) VDAC2 quantification on the mitochondrial membrane during short term starvation. Cells were grown to $90 \%$ confluency and starved for 4 hours, followed by whole lysate extraction or mitochondria purification. Western blot quantification shows the ratio of mitochondrial VDAC2 to mitochondrial COX4 levels.

(C) VDAC2 clustering is regulated by GSK3 kinase. Cells with endogenous or over-expressed VDAC2 were incubated with arsenite $(100 \mu \mathrm{M})$ or with arsenite in the presence of GSK3 inhibitors

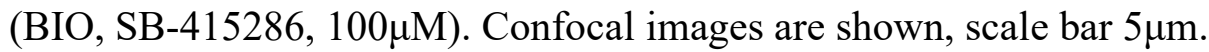


(D) Analysis of SG formation. Cells expressing PABPC1-DDR2 were starved for 2 hours, then treated for 1 hour with 1-NA-PP1, SMI-4a, AS-703026, ruxolitinib, KN-93, TGX-221, AG-17 $(100 \mu \mathrm{M}$ each) and visualized. Representative confocal images are shown, scale bar $5 \mu \mathrm{m}$.

\section{Figure S8.}

(A) Transmitted light microscopy of embryoid body formation during 5 days of differentiation in control and TDP43 mutant human iPSCs.

(B) Quantification of stem cell and neuronal lineage marker expression using RT-PCR, mean \pm SEM.

(E) Confocal microscopy of FA accumulation during starvation. Human iPSC differentiated to Neuronal progenitors were starved in a fuel and serum depleted media containing FA-free BSA for indicated amounts of time, FA dye (Bodipy-C12, $1 \mu \mathrm{M}$ ) was added 30 minutes prior to the imaging together with Hoechst $(10 \mu \mathrm{g} / \mathrm{ml})$.

(F) Analysis of FAO during starvation. Human iPSC differentiated to neuronal progenitor cells with or without TDP43 mutation were starved for 2 hours with or without FA import inhibition and analyzed using Seahorse Mito Fuel Flex test. Graph represents fatty acid dependency, mean \pm SEM. 


\section{Figure 1}
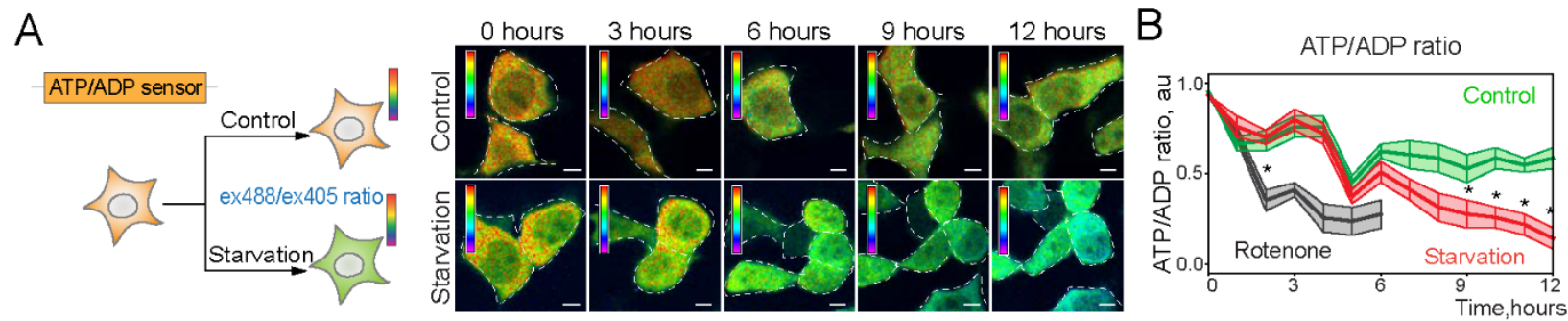

$$
\text { C }
$$

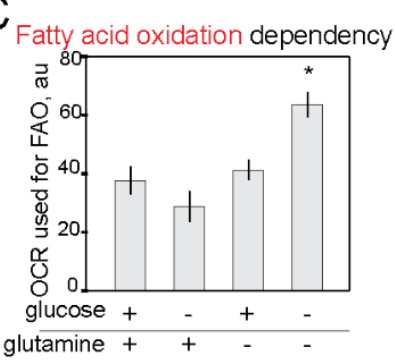

D
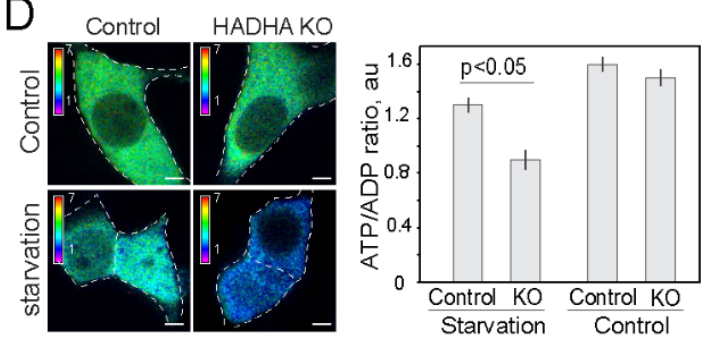

E

G
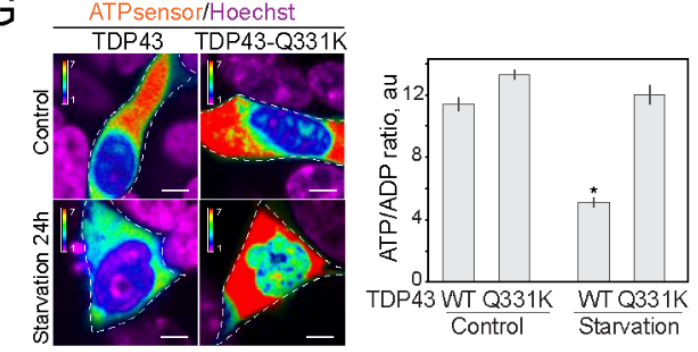

$\mathrm{H}$ Oxidative stress/H2-DFCDA

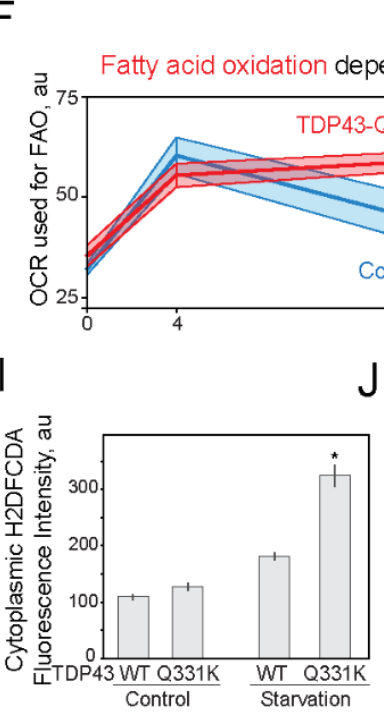

TDP43-Q331K
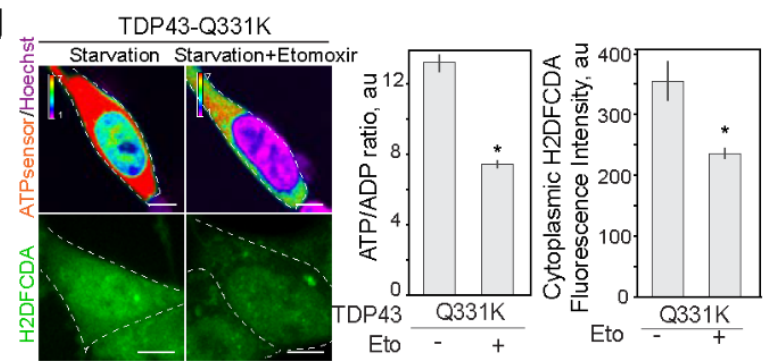

K

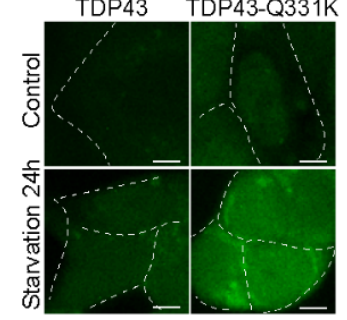

L

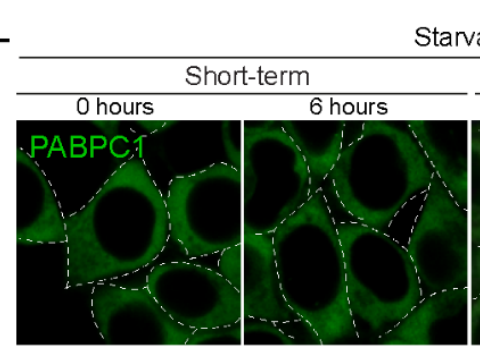

M

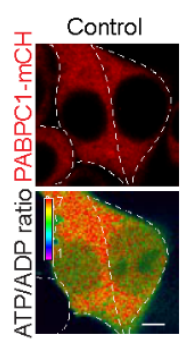

Arsenite

Starvation Rotenone, CCCP
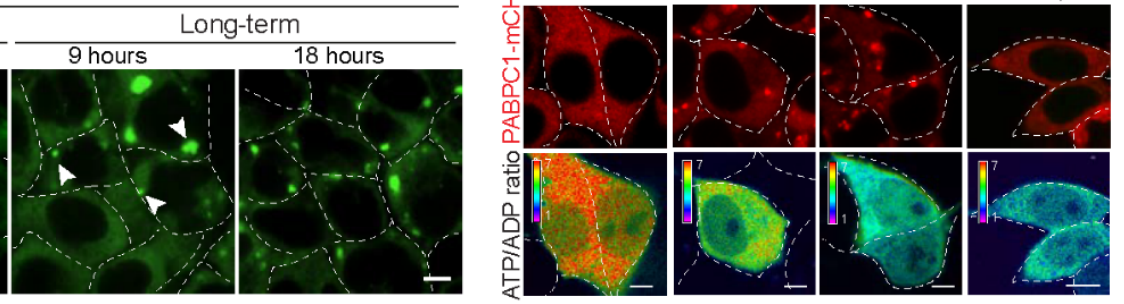


\section{Figure 2}

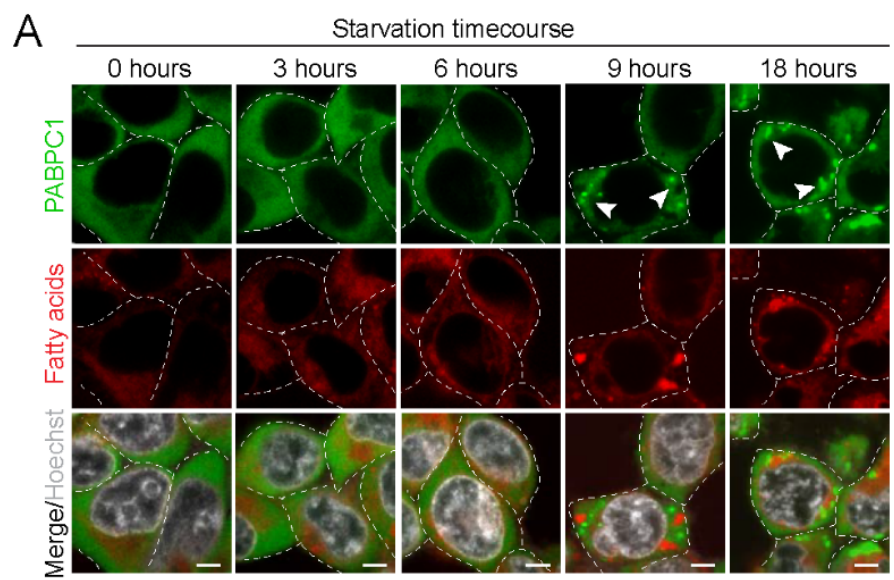

B

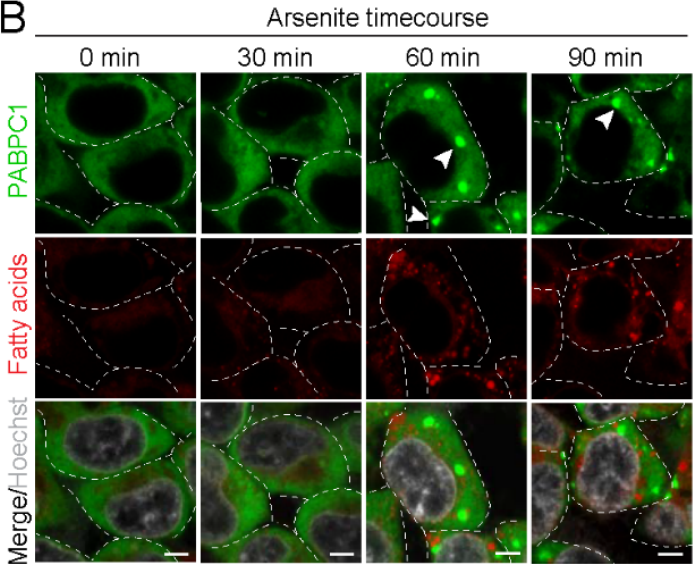

C

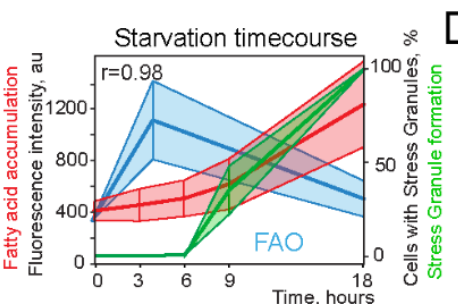

$F_{3}$
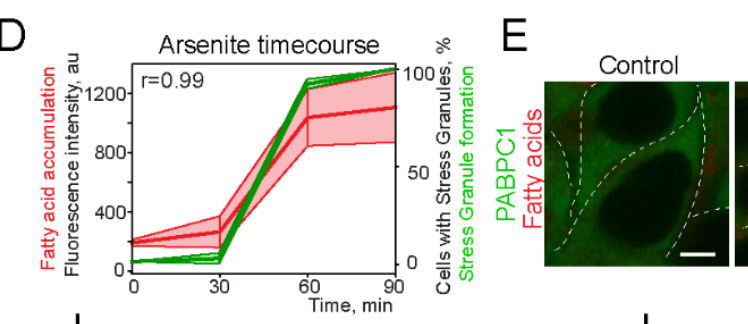

Arsenite
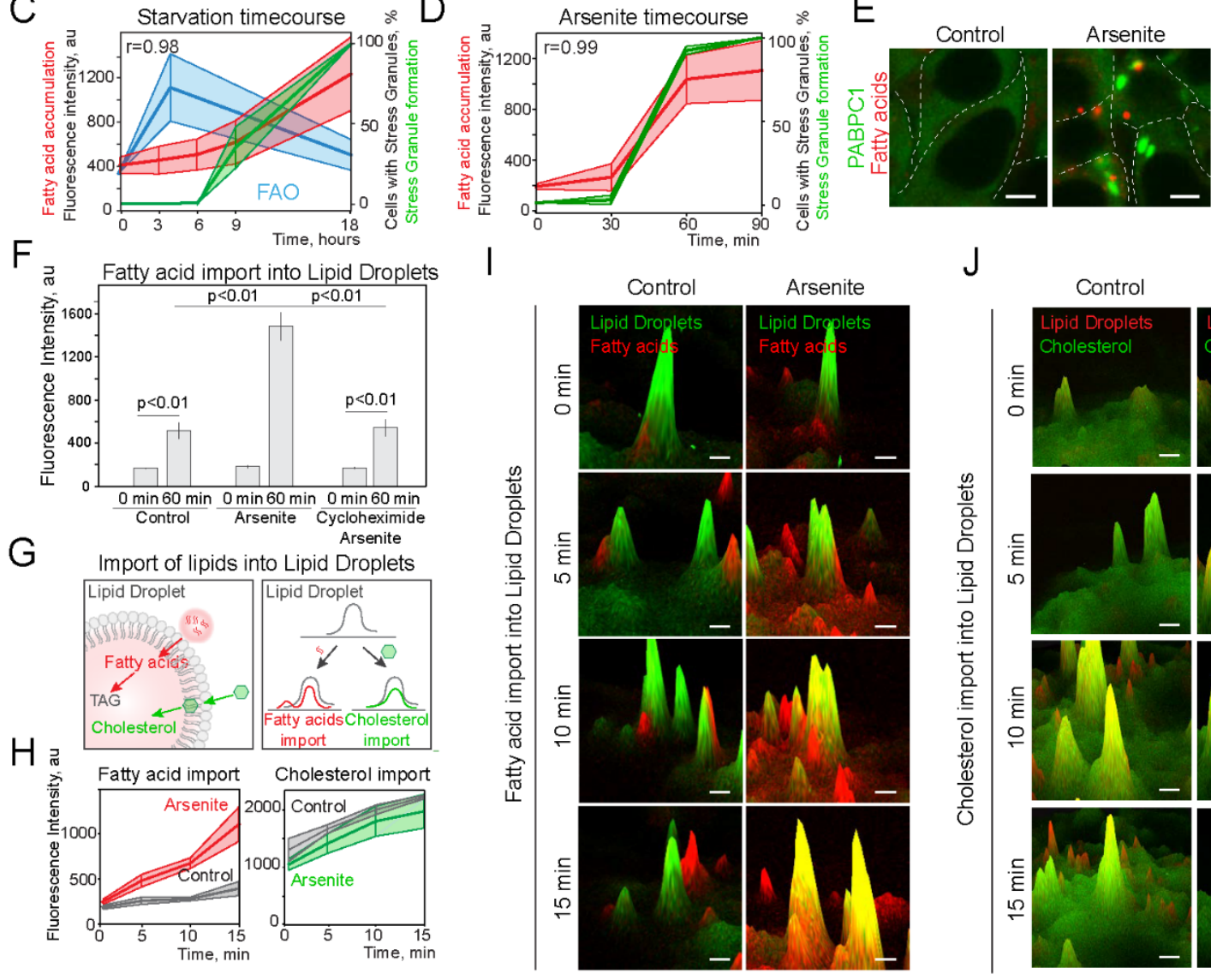

Arsenite

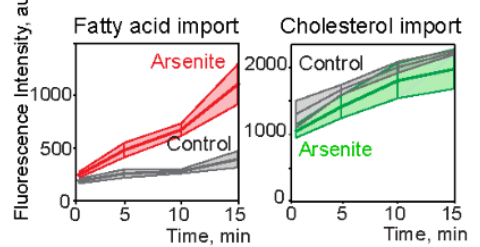

K
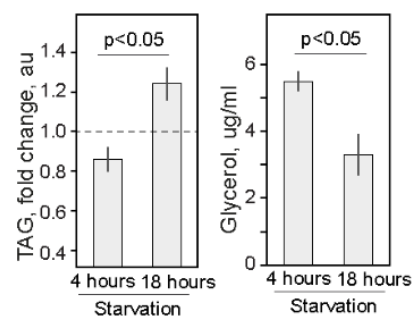

L

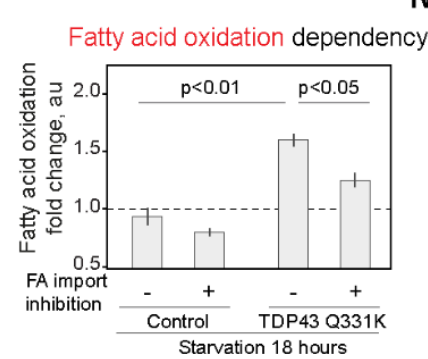

M

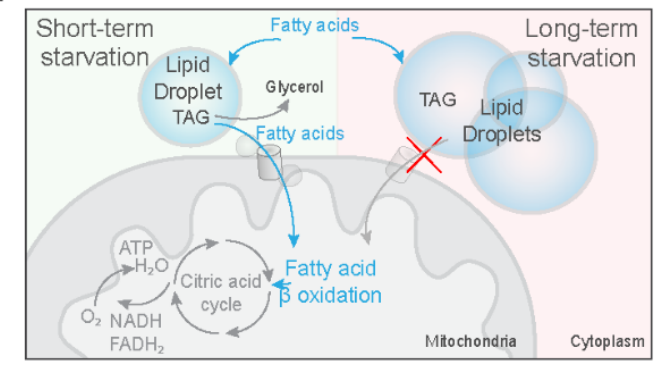


Figure 3

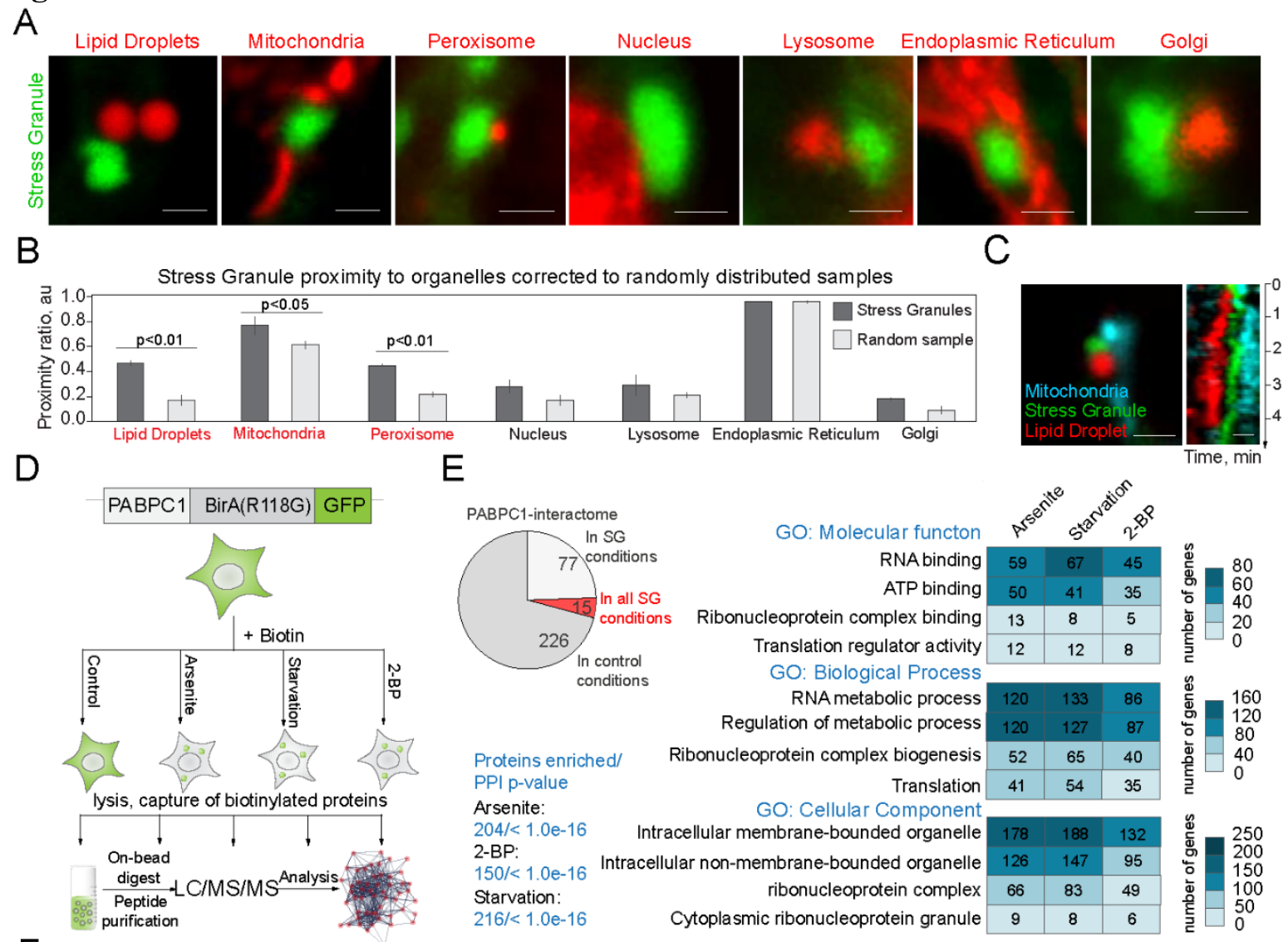

F

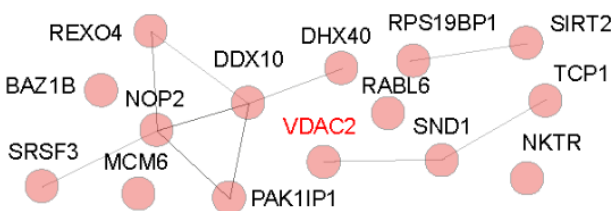

$\mathrm{H}$

PABPC1 $\mathrm{mCH}$ VenusC

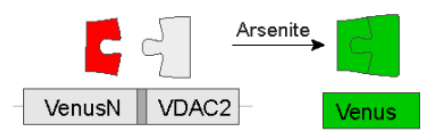

I

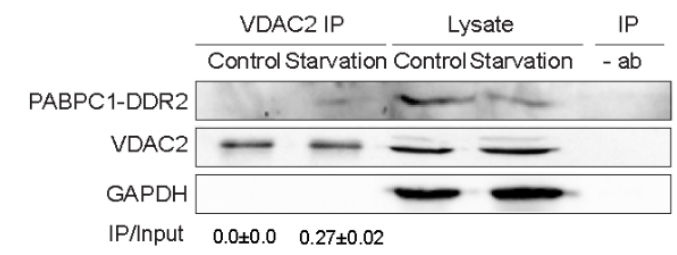

K

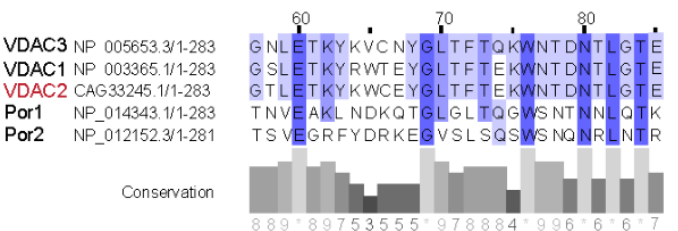

G

Overlap with G3BP (Isabelle et al., 2012)

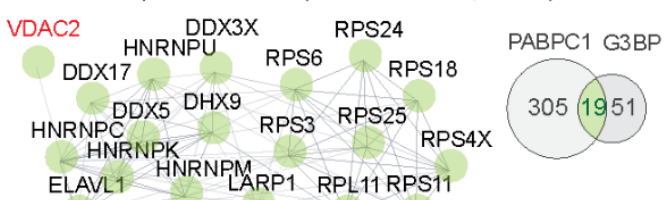

$J$

Proteins shared with Lipid Droplets (Roesch et al., 2016)

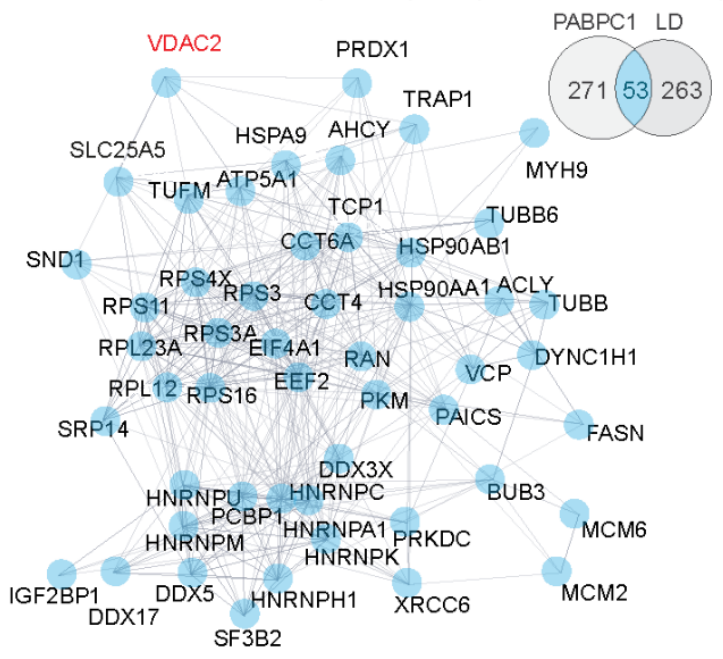




\section{Figure 4}
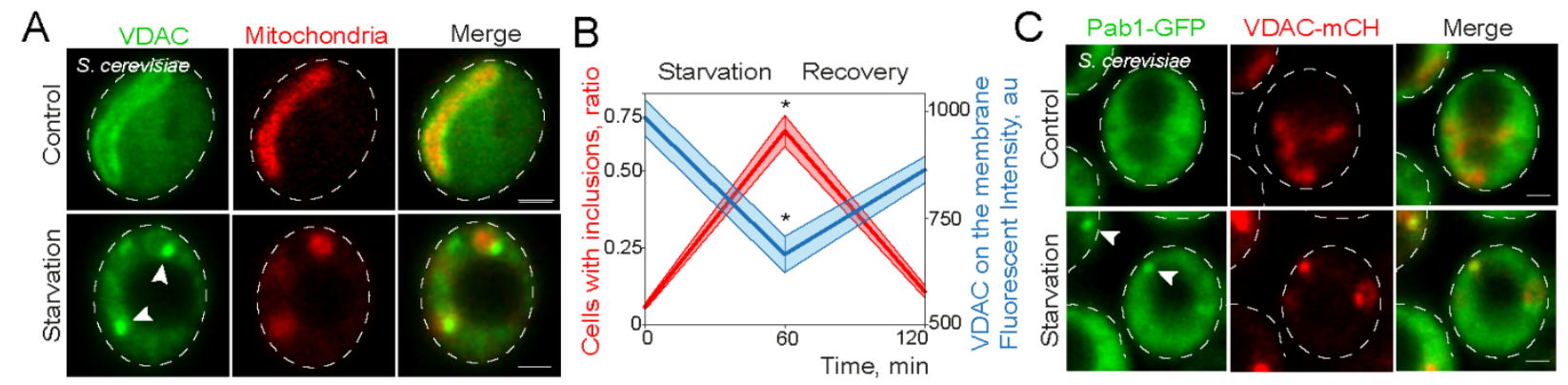

D

Starvation

Recovery
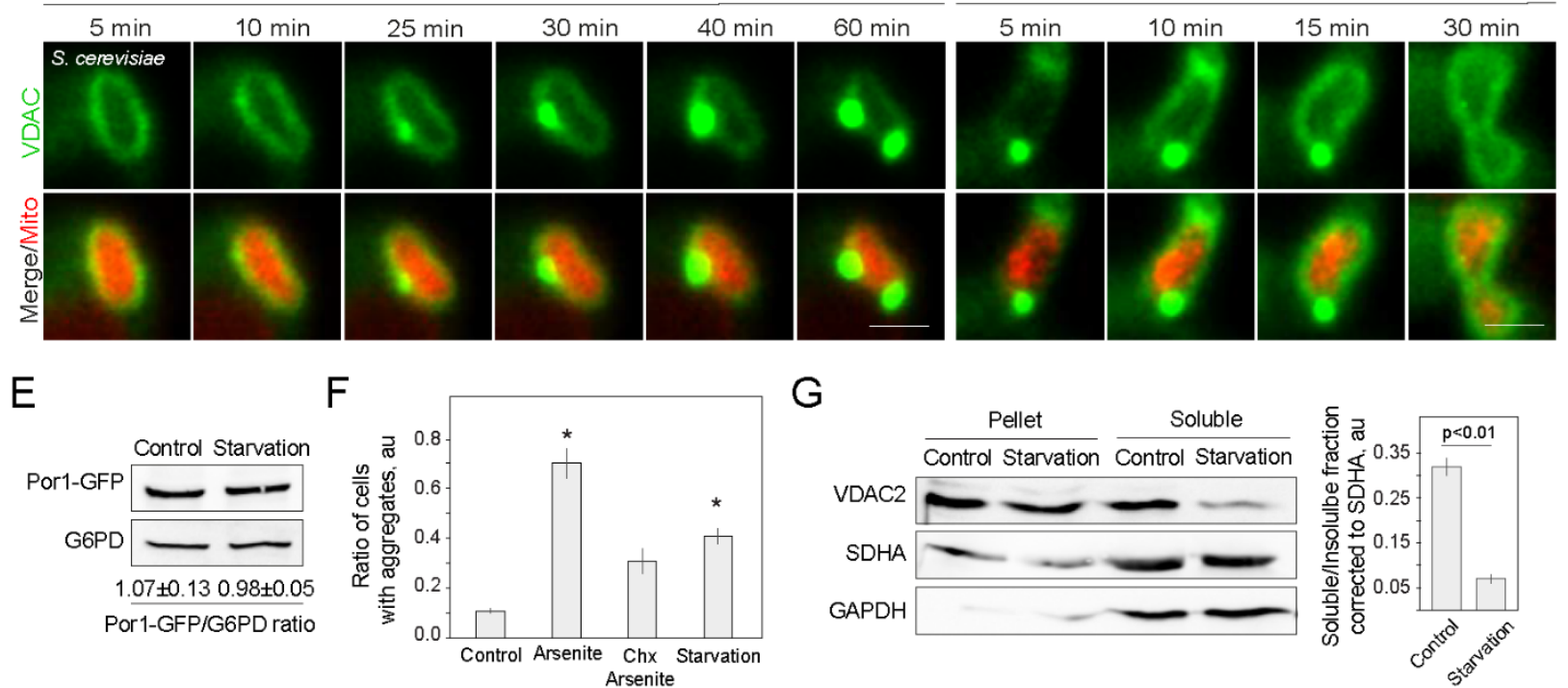

G

$\mathrm{H}$

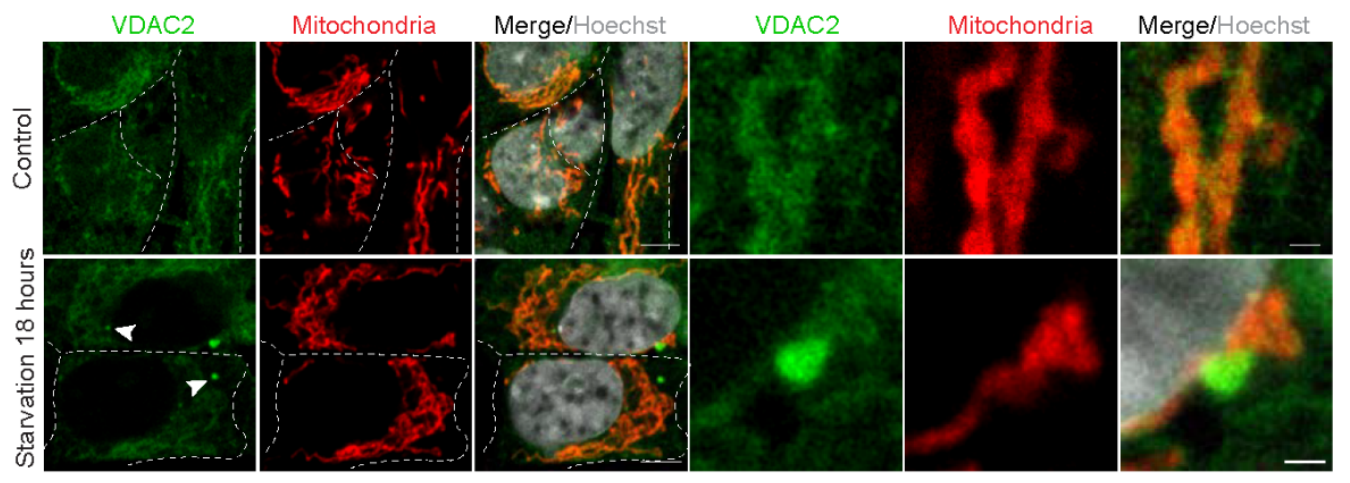




\section{Figure 5}
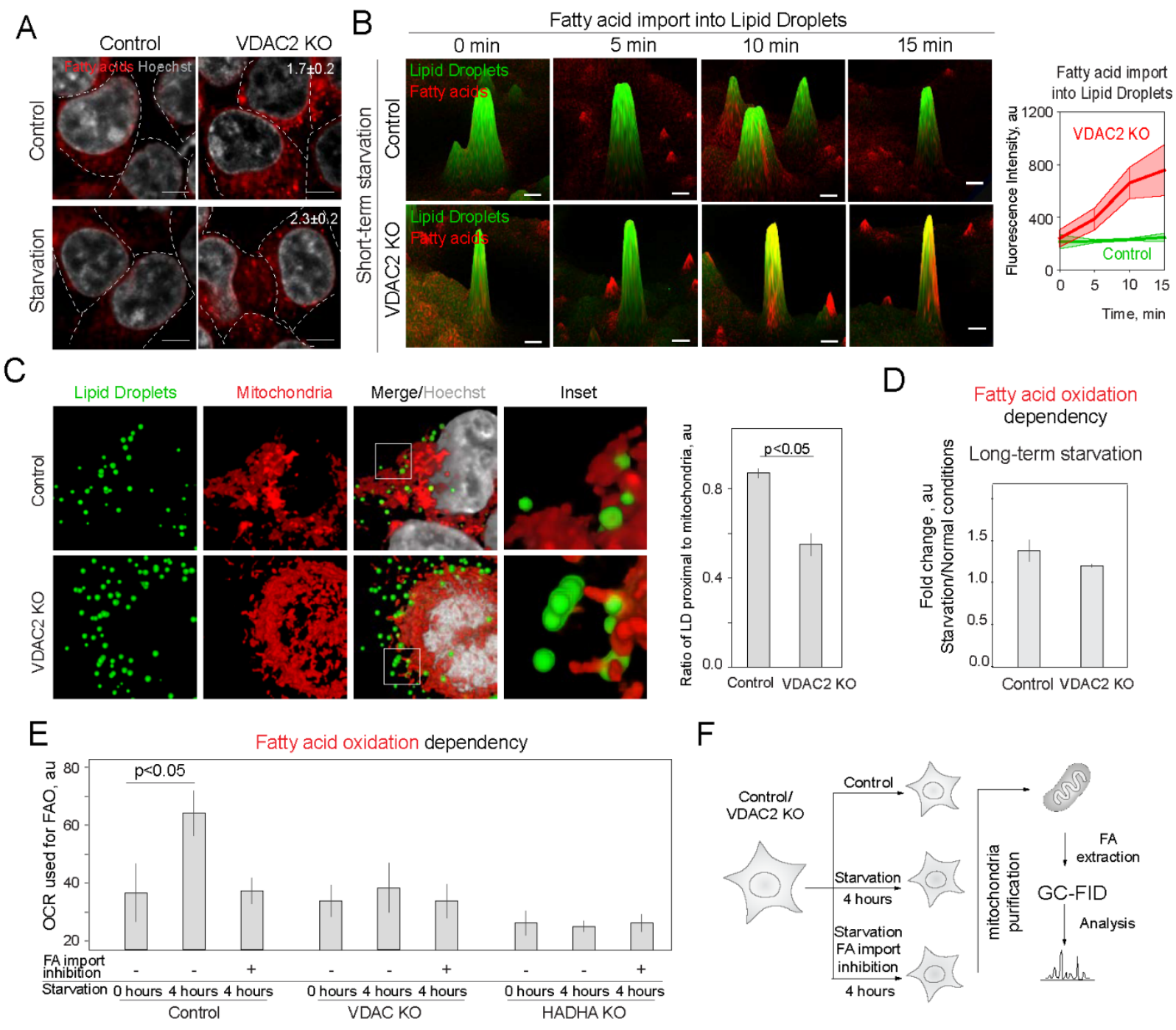

D Fatty acid oxidation

F

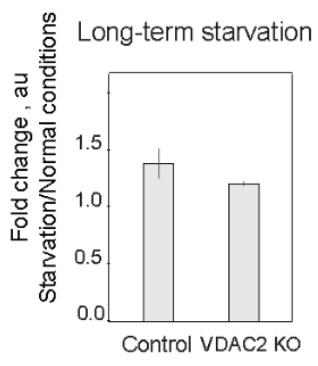

G

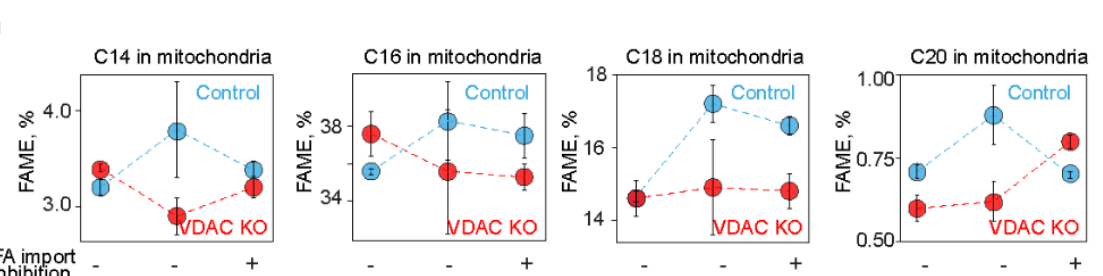

Starvation 0 hours 4 hours 4 hours $\quad 0 \overline{\text { hours } 4 \text { hours } 4 \text { hours }} \quad 0 \overline{\text { hours } 4 \text { hours } 4 \text { hours }} \quad \overline{0 \text { hours } 4 \text { hours } 4 \text { hours }}$
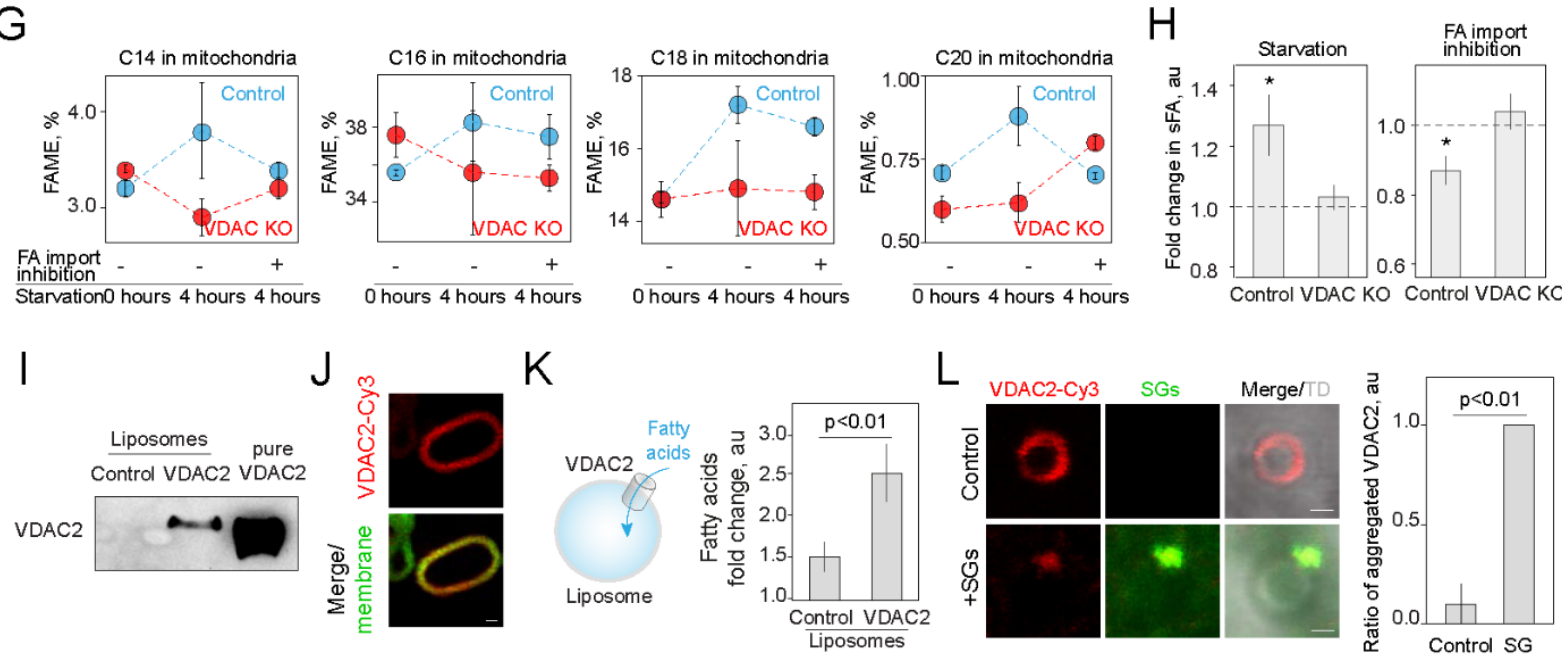


\section{Figure 6}

A
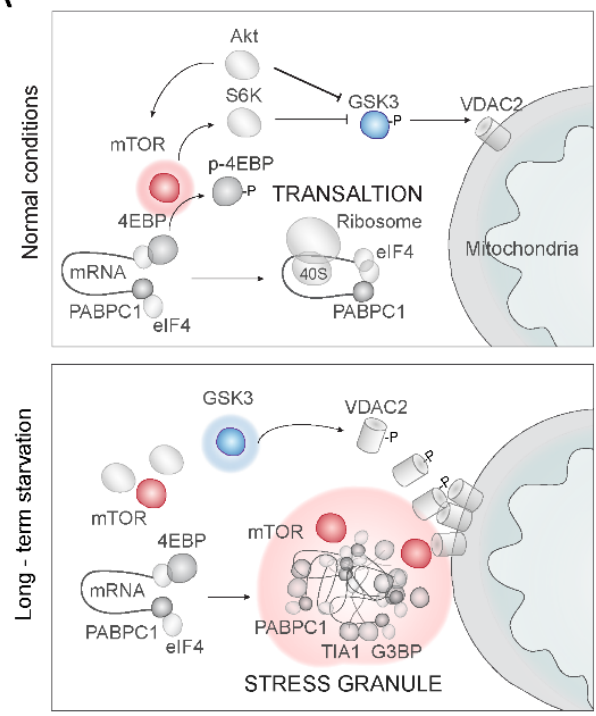

F
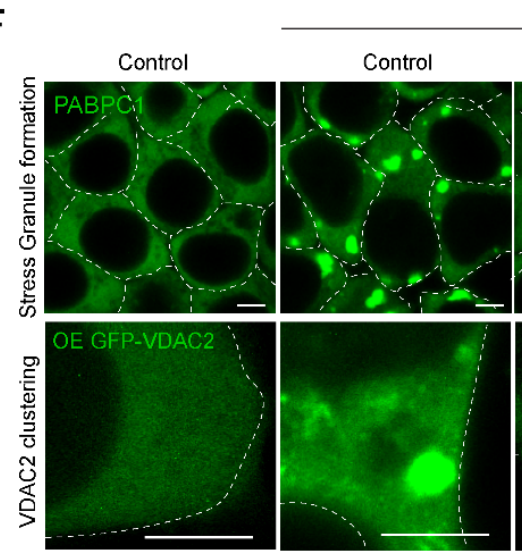

B

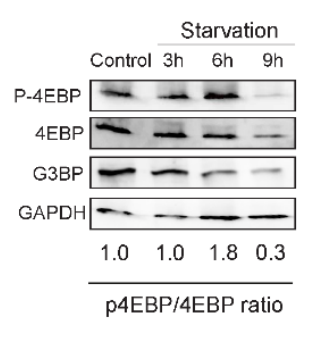

D

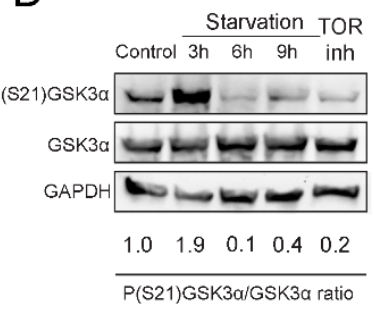

Arsenite
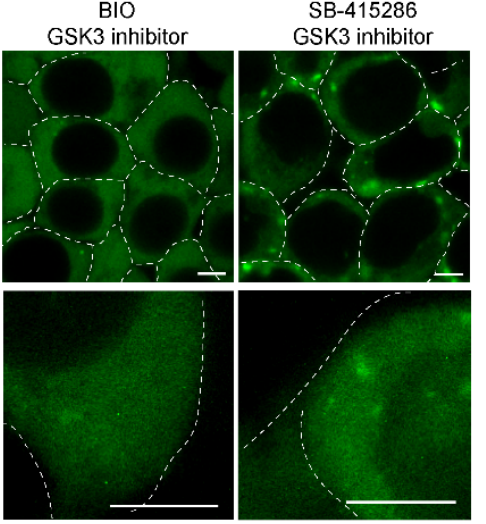

C

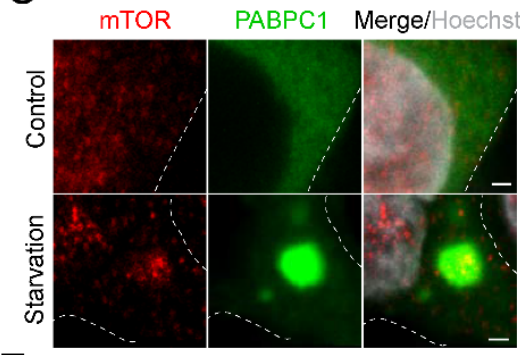

E

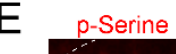

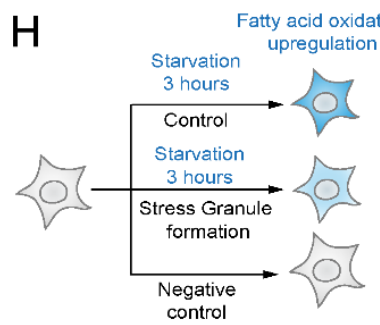
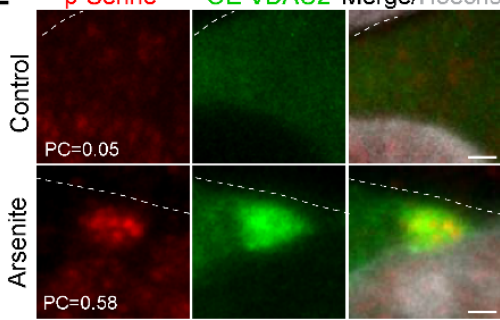

G
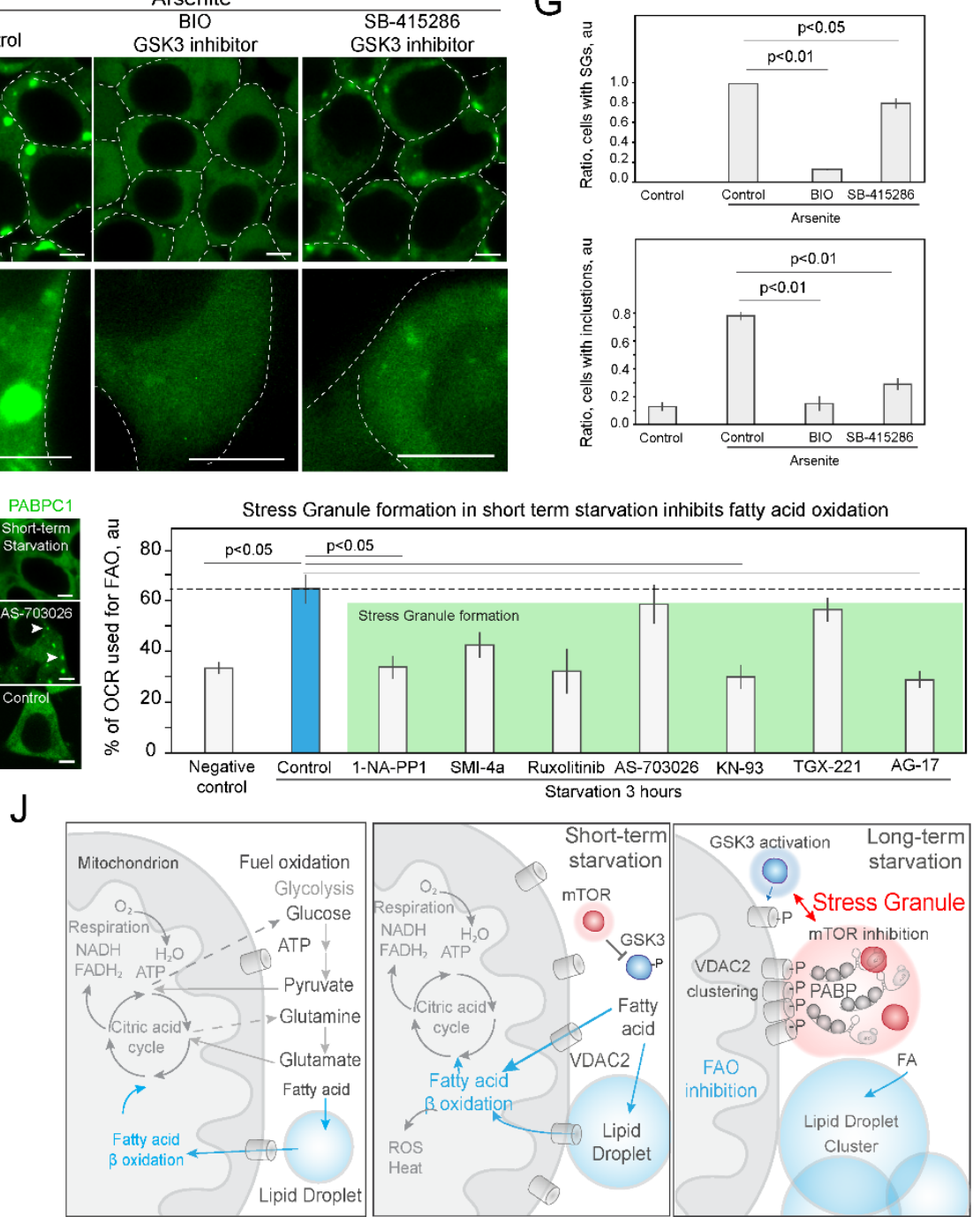


\section{Figure 7}

A

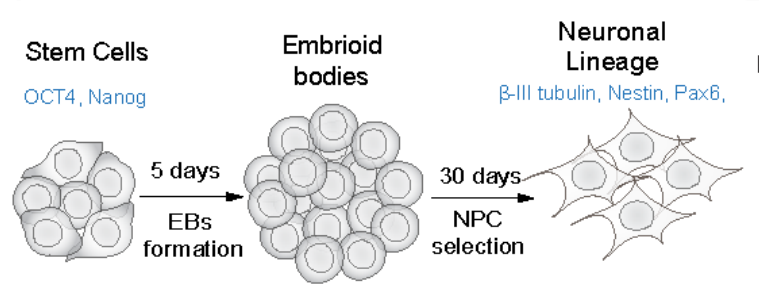

D

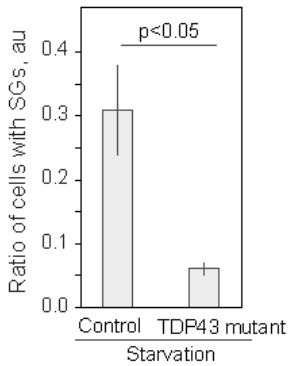

E

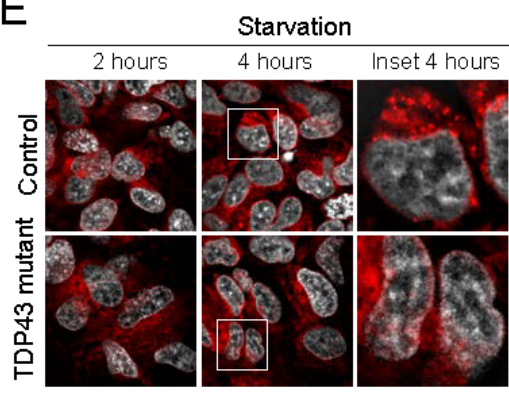

B C

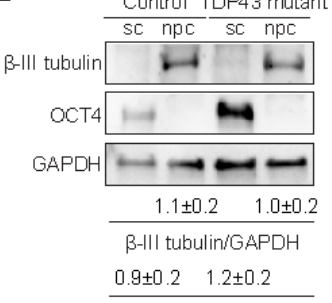

$\frac{0.9 \pm 0.2 \quad 1.2 \pm 0.2}{\text { OCT4/GAPDH }}$

\section{$\mathrm{F}$}
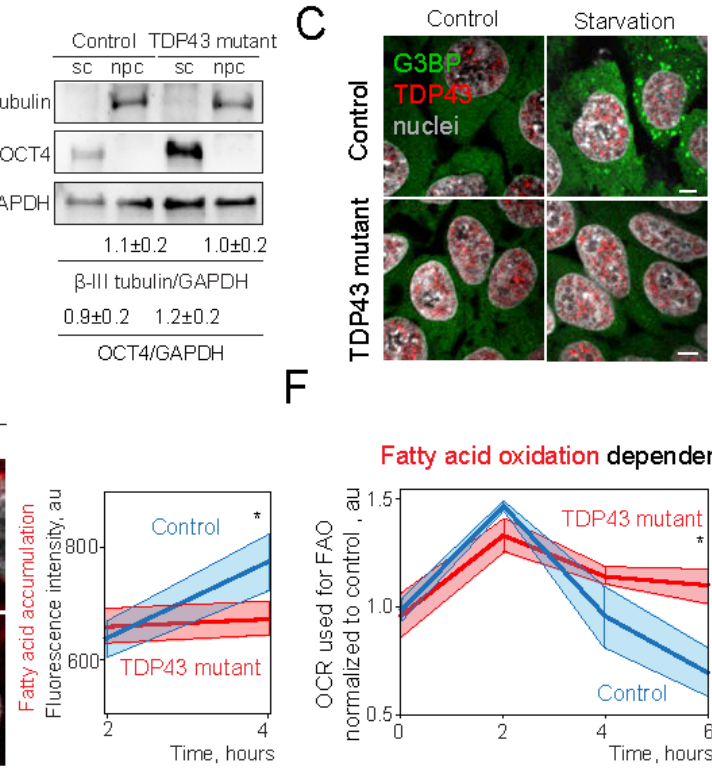

Fatty acid oxidation dependency

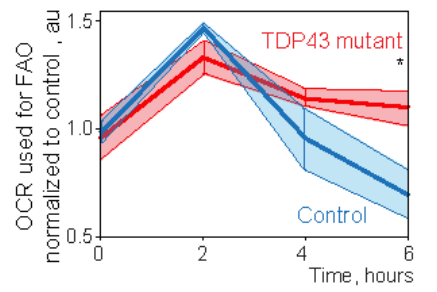




\section{Figure S1}
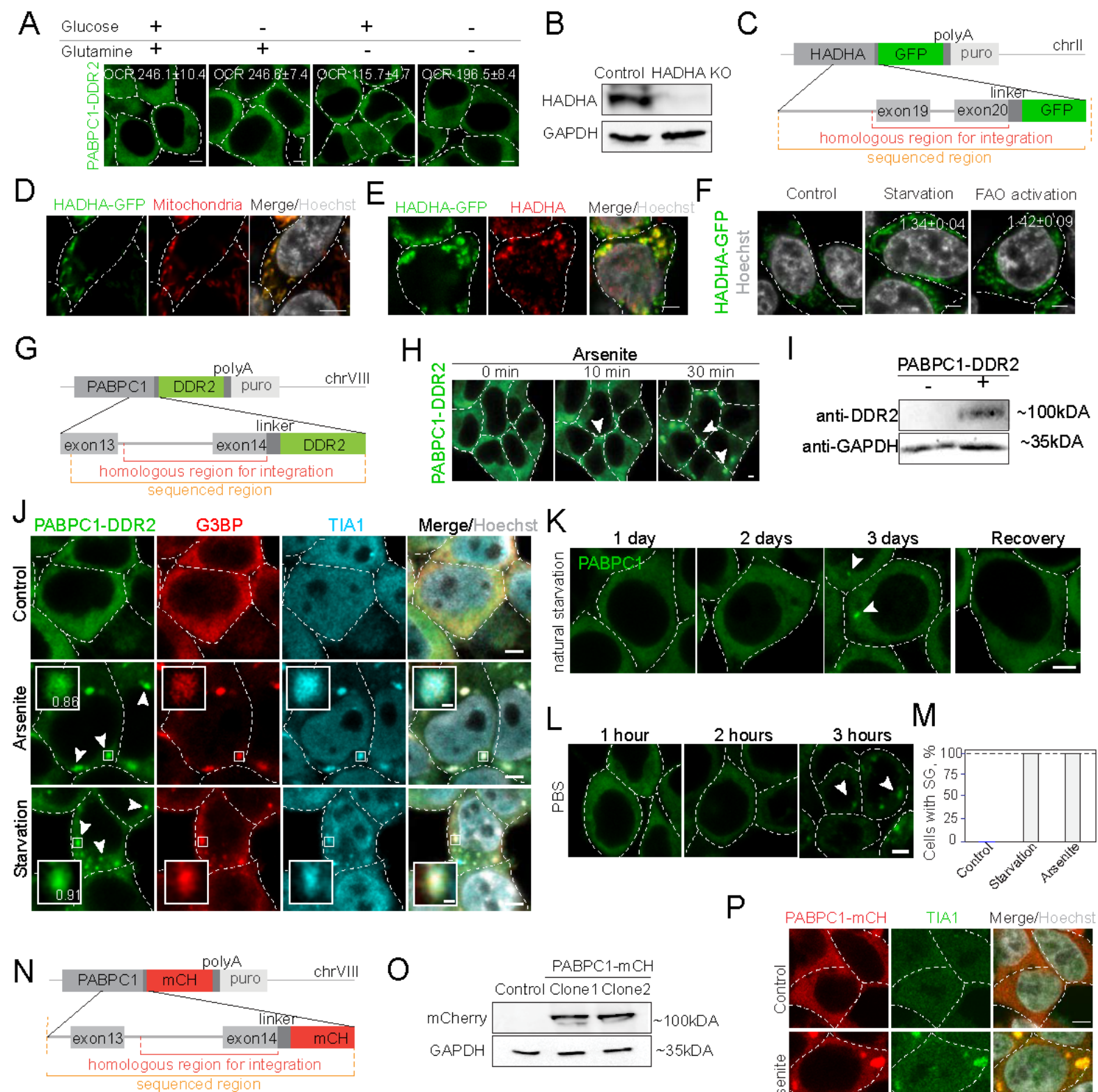

Q

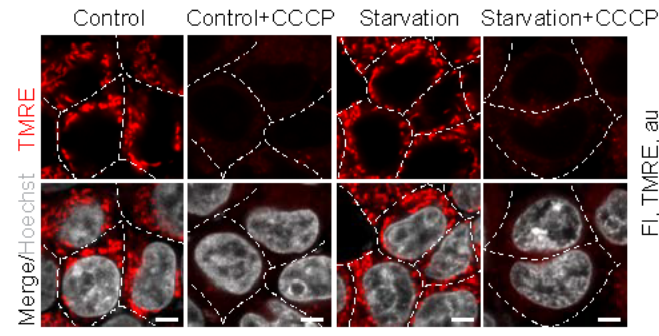

$\mathrm{R}$

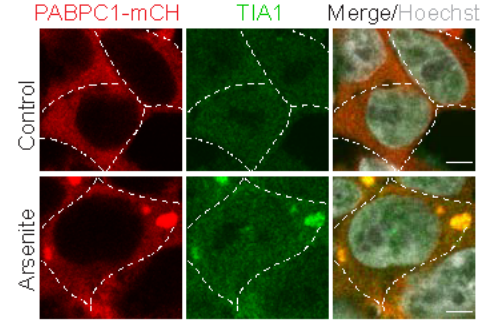

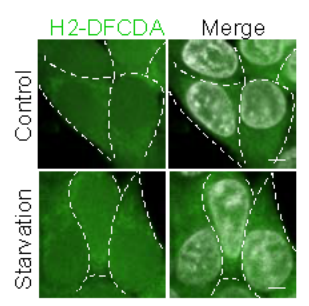

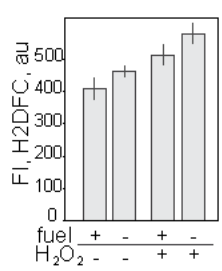




\section{Figure S2}

A

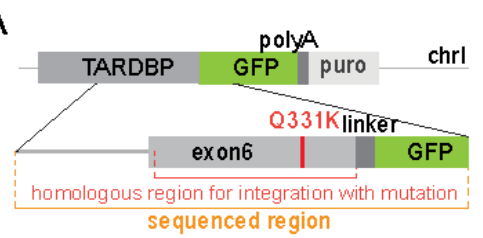

B

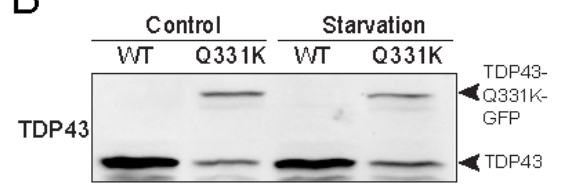

GAPDH $\longrightarrow \sim$
C
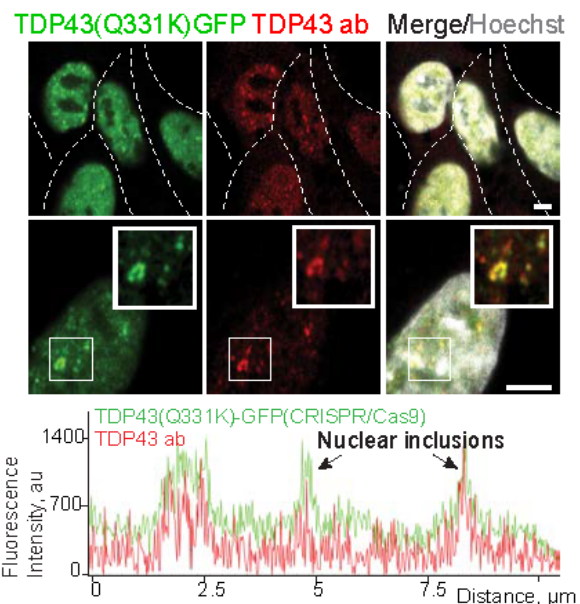

E

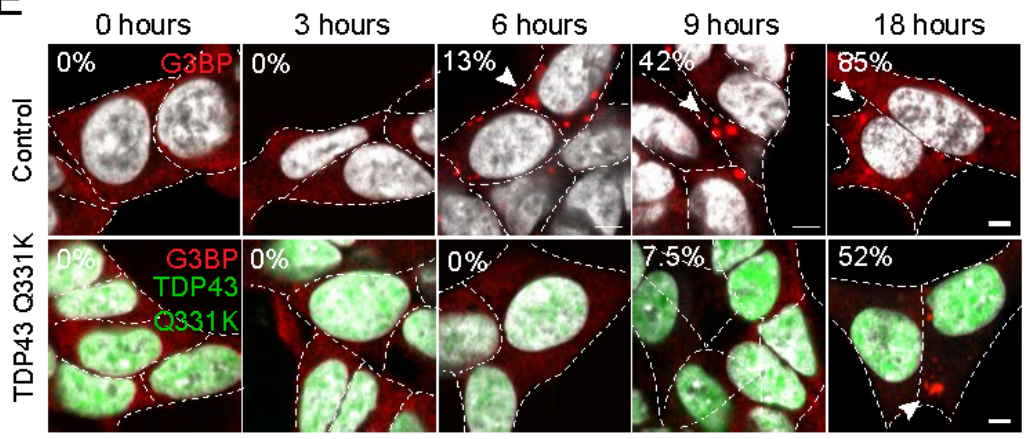

D

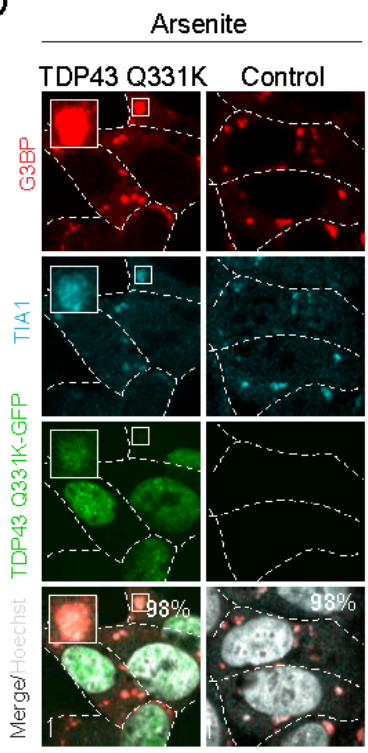

F TDP43-Q331K

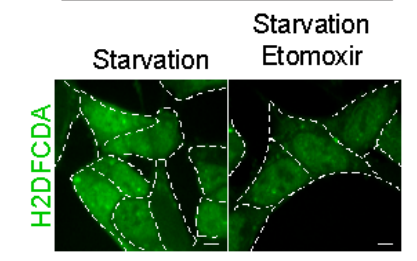

G

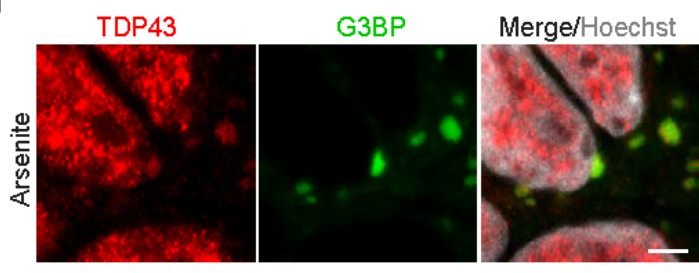

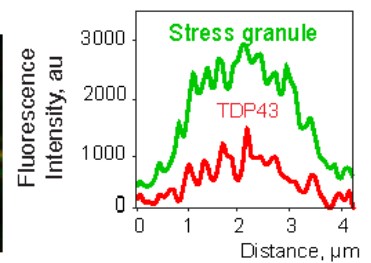




\section{Figure S3}

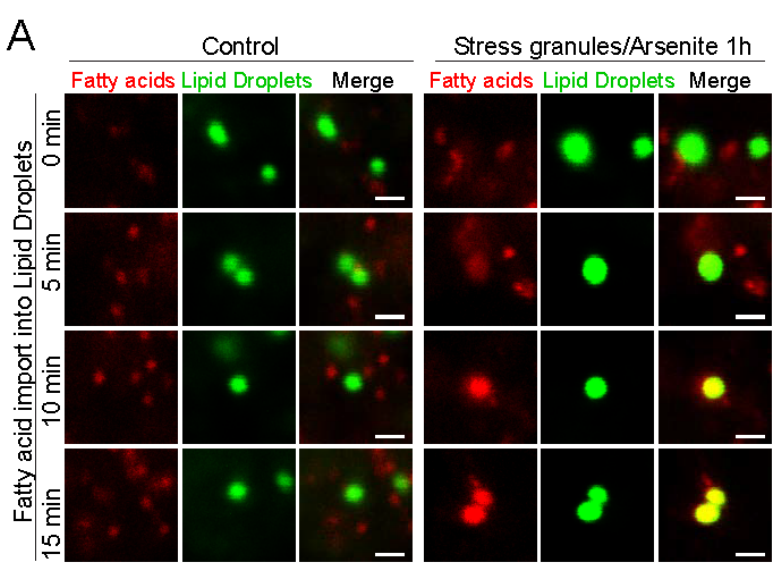

C Control Arsenite Arsenite Cycloheximide D

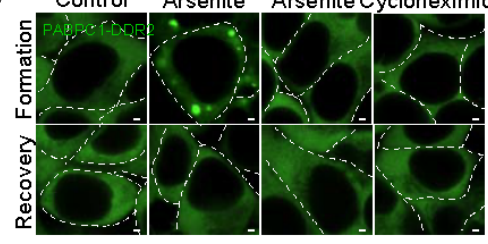

F

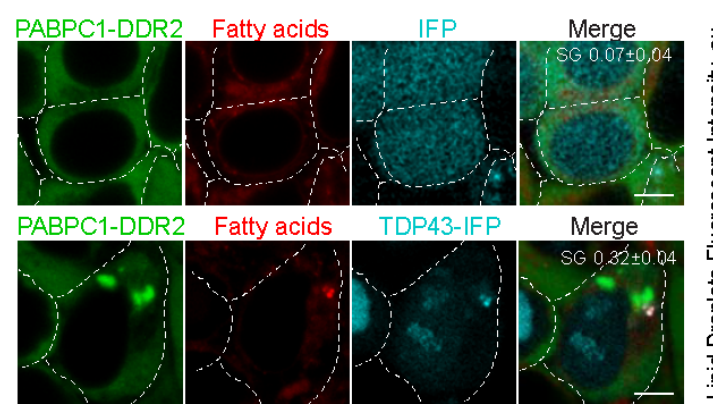

B
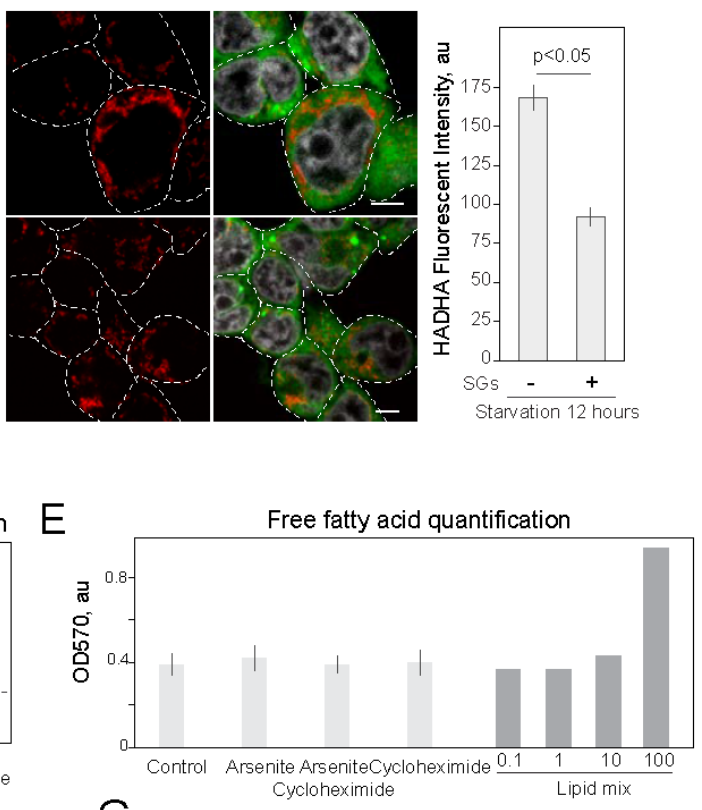

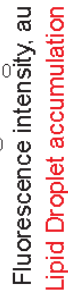

G
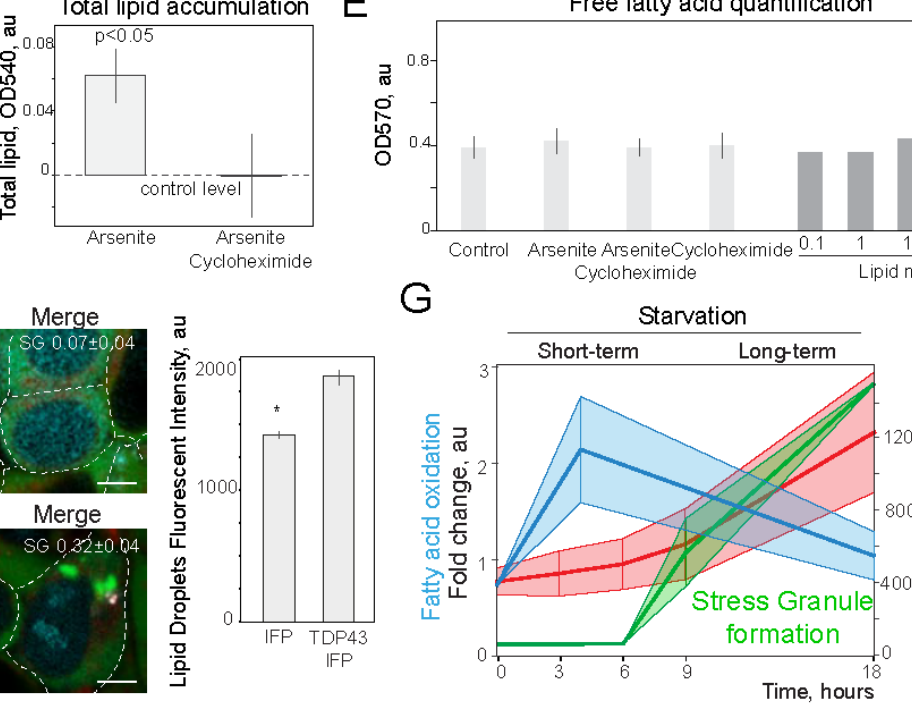


\section{Figure S4}
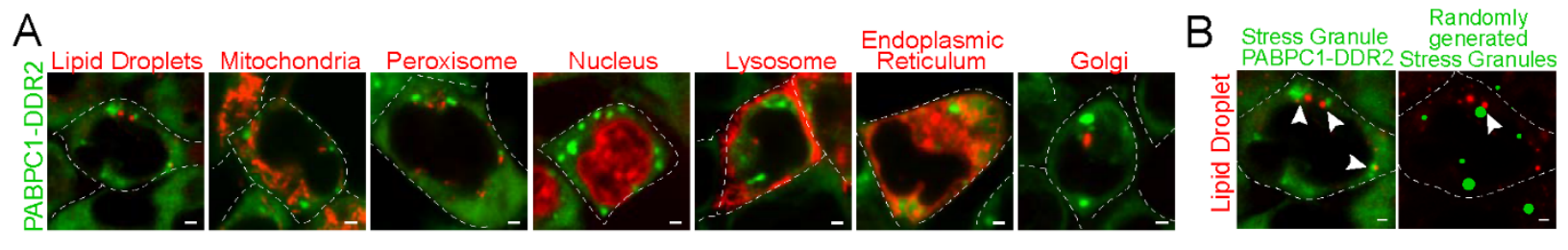

C

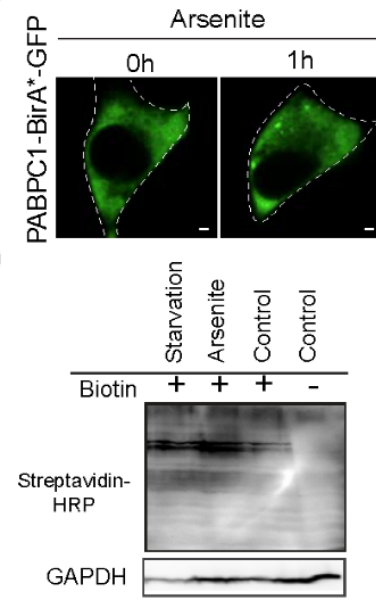

E

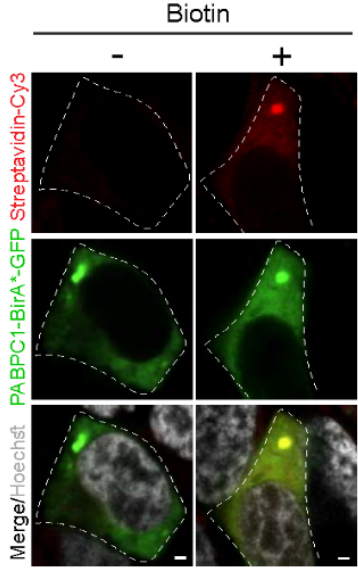

G Overlap with Stress Granule proteome (Jain et al., 2012)

$$
\begin{aligned}
& \begin{array}{l}
\text { HSPA1A } \\
\text { SRRM1 } \\
\text { TUBB SRRM2DHX9 } \\
\text { ACIN1 }
\end{array} \\
& \text { BCLAF1 } 1 \text { NRNPC ELAVL1 XRCC6 SMARCA5H2AFY } \\
& \text { NUMA1 PCBP1 DDX55 EIF4A3 NOP56 DDX21 BAZ1B } \\
& \text { HNRNPH1 NOP56 DDX21 BAZ1B } \\
& \text { PGAM5 DHX30 }{ }_{\text {DDX17 }} \text { FTSJ3 IGF2BP1 } \\
& \text { DDX47 RPL4 RPS8 SLC25A5 }
\end{aligned}
$$

Overlap with FUS interactome (Kamelgarn et al., 2016)

$$
\begin{aligned}
& \text { DDX3X DHX30 PARP1 SRSF7 PABPC1 FUS } \\
& \begin{array}{llll}
\hline \text { LARP1 } & \text { DDX5 } & \text { DHX9 } & \text { DDX17 } \\
\hline
\end{array}
\end{aligned}
$$

Overlap with TDP43 interactome (Freibaum et al., 2010)

$$
\begin{aligned}
& \text { VCP TUBB MYH9 SLC25A5 }{ }^{\text {ATP5A1 }} \text { HIST1H1D SRBD1 } \\
& \text { HSPATA RPL10 H1FX PGAM5 }
\end{aligned}
$$

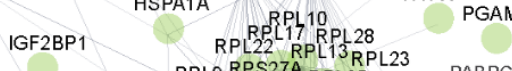

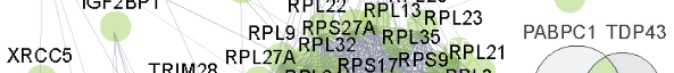

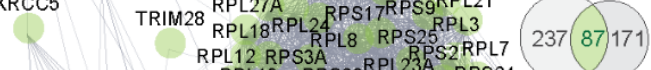

$$
\begin{aligned}
& \begin{array}{l}
\text { RPL12 RPS3A } \\
\text { RPL19 RPS RPL } 23 A^{2} \text { RP RPL } 24 \\
\text { RP } 23
\end{array}
\end{aligned}
$$

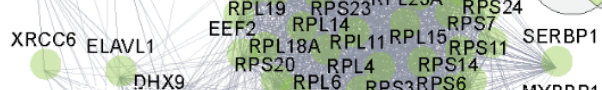

$$
\begin{aligned}
& \begin{array}{lll}
\text { HNRNPHX9 } & \text { RPL6 RPS3RPS6 } & \text { MYBBP1A }
\end{array} \\
& \text { KPNA2 PCBP1 HNRNPA1 } 1 \text { A3 } \\
& \text { SART HNRNPMNRP2 EIF4A1 FBL TSR GNL }{ }^{\text {DDX50 }} \\
& \text { HNRNPH1 DDX5 DDX21 SND1 DHX30 } \\
& \text { LUC7L2 TOP1 }{ }_{\text {RBM } 39}^{\text {DDX17 SRPK1 }}{ }_{\text {DDX3X LARP1 NOP16 }}
\end{aligned}
$$

$\mathrm{F}$
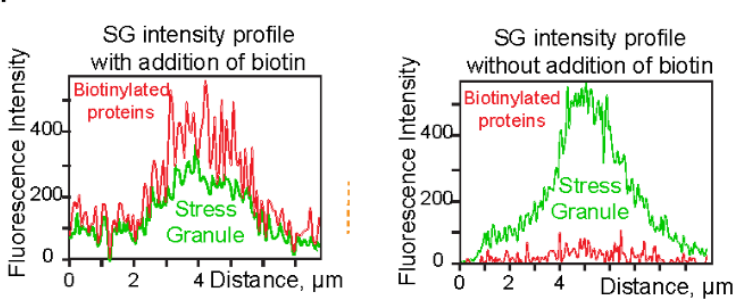

$\mathrm{H}$

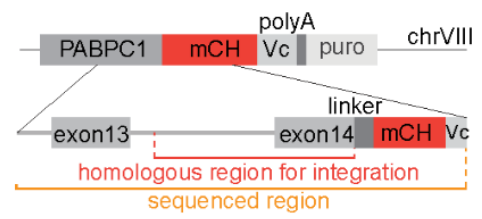

।

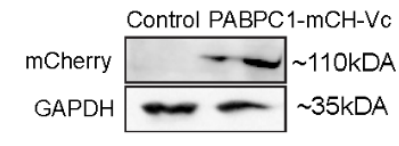

$J$

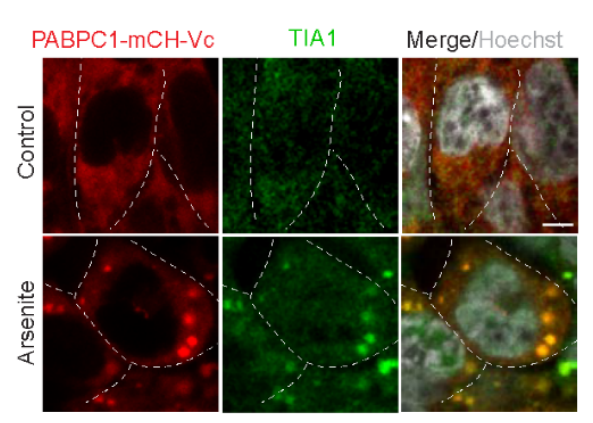

K

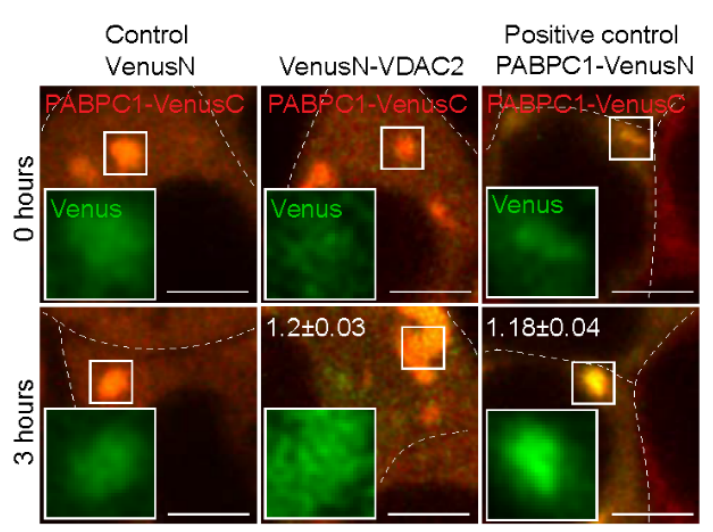




\section{Figure S5}

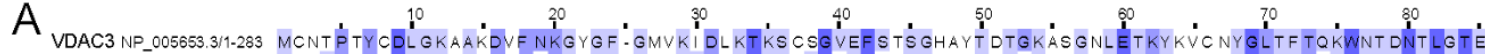
VDAC1 NP_003365.111-283 MAVPPTYADL GKS ARDVFTKGYGF. GL I KL DL KT KSENGLEFTSSGSANTETTKVT GSLETKYRWTEYGLTFTEKWNT DNTLGTE DAC2 CAG33245.1/1-283 MCIPP SYADL GKAARDIFNKGF GF - GLVKL DVKT KS CSGVEFSTSGSSNTDTGKVTGTLETKYKWCEYGLTFTEKWNT DNTLGTE Por2 NP.012152.311-281. -MALRFFNDI SRDVNGLFNRDFFHTNPLSLNISTTTENGVNFTLKAKOGVTEGPIQTSVIGRFYDRKEGVSLSQSWSNQNRLINTR

Conservation

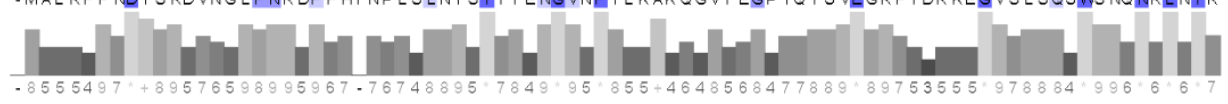

9.0 100

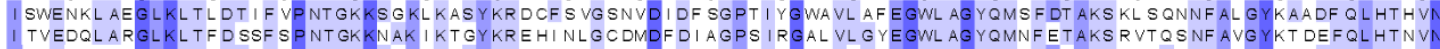
IAIEDQICQGLKLTFDTTFSPNTGKKSGKIKSSYKRECINLGCDVDFDFAGPAI HGSAVFGYEGWLAGYMTFDSAKSKLTRNNFAVG YRTGDFQLHTNVN

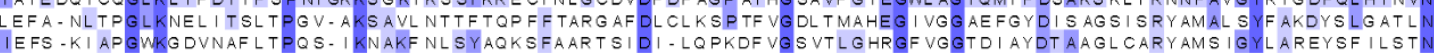

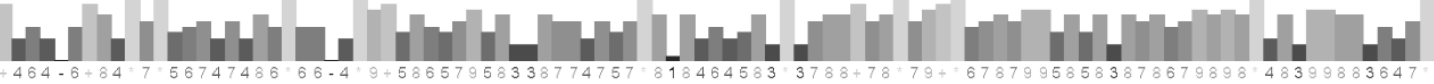

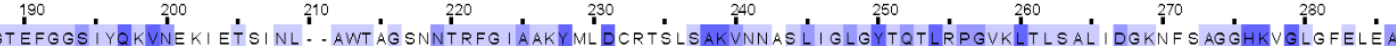
DGTEFGGSIYOKVNKKLETAVNL . AWTAGNSNTRFGIAAKYO I PDACFSAKVNNSSLIGLGYTOTLKPGIKLTLSALLDGKNVNAGGHKLGLGLEFO DGTEFGGSIYOKVGEDLDTSVNL. AWTSGTNCTRFGI AAKYQLDPTASISAKVNNSSLIGVGYTOTLRPGVKLTLSALVDGKSINAGGHKVGLALELE NEO ITTVDFFONVNAFLQVGAKATMNCKLPNSNVNIEFATRYLPDASSOVKAKVS DSGIVTLAYKOLLRPGVT GVGSSFDALKLSEPVHKLGWSLSFD NRQCAT ASFFQNVNRYLQVGTKAT LQSKT. SSNMNIEFVTRYVPDSI SQVKAKI ADSGLT TLSYKRNLNKDI SLGVGMSFNALQLTEPVHKFGWSLSFSP

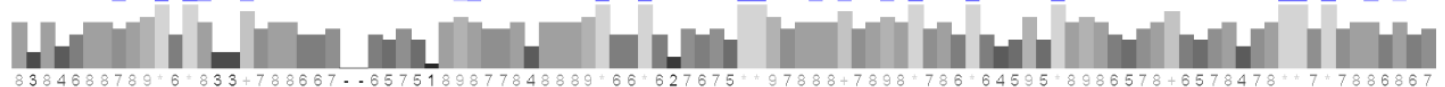

B

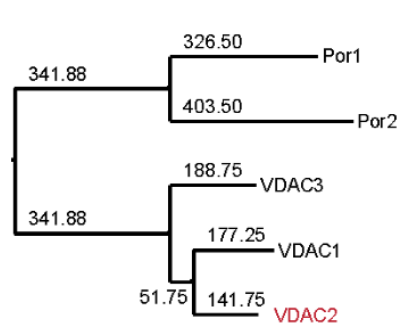

D

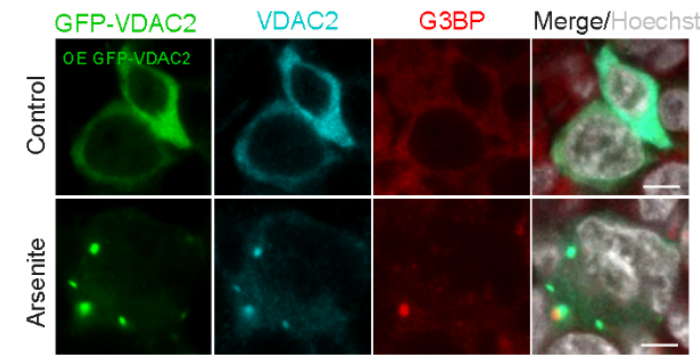

E

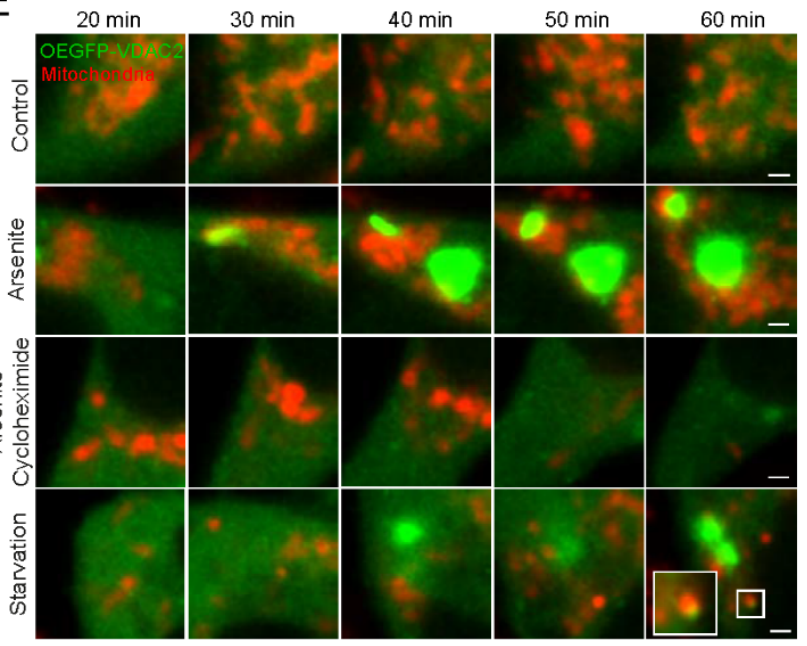

C $\quad \underset{P a b 1-G F P}{\mathrm{MITO}} \begin{array}{llllll}0 & 1 & 2 & 3 & 4 & \text { 5Time, min }\end{array}$
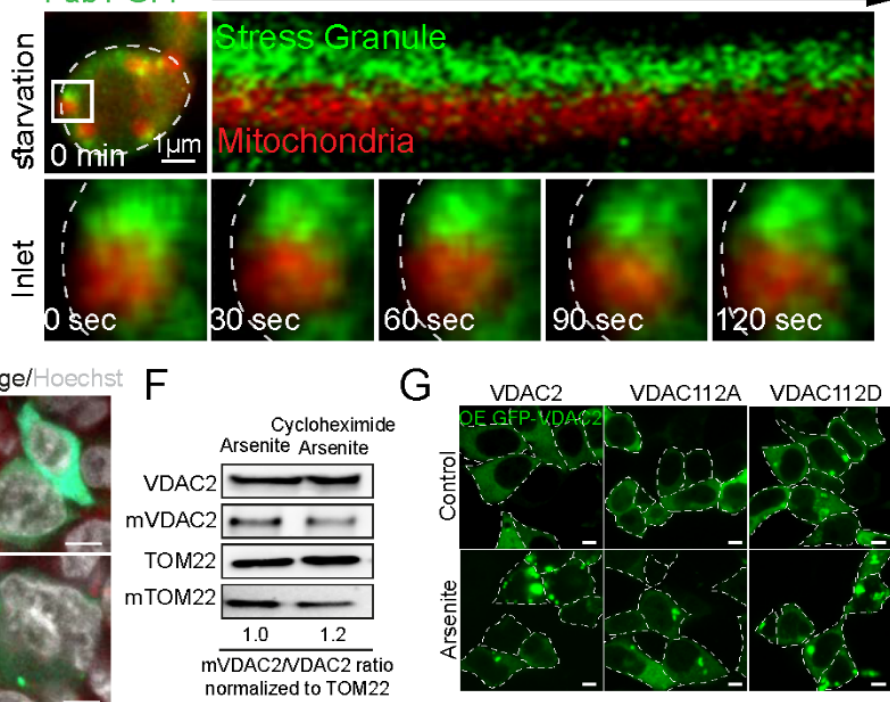

G
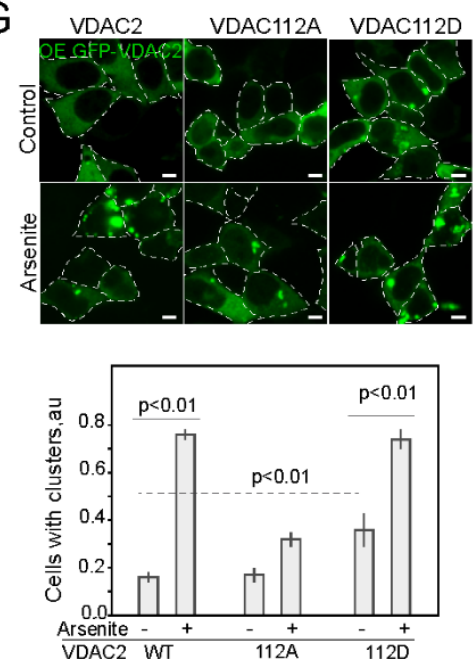

$\mathrm{H}$

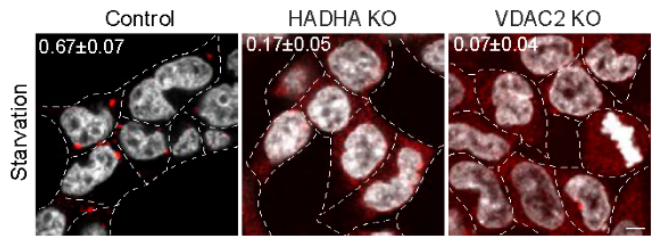




\section{Figure S6}

A

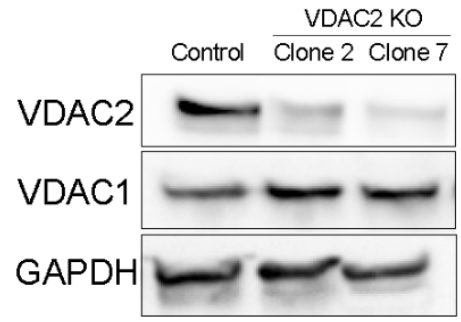

VDAC2

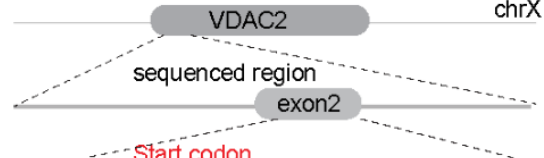

WT GCCATGGCGACCCACGGACAGACTTG Clone\#2 GGCYTSSCCHRGKSGMCCCMSGGVMR

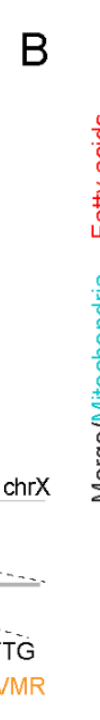

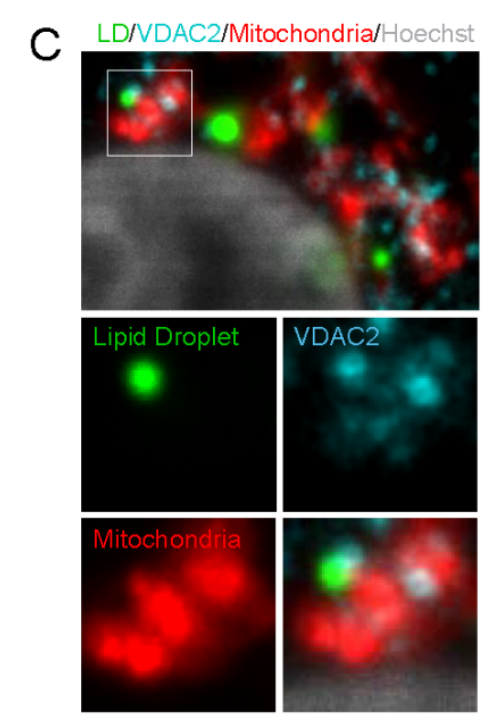

$\mathrm{F}$

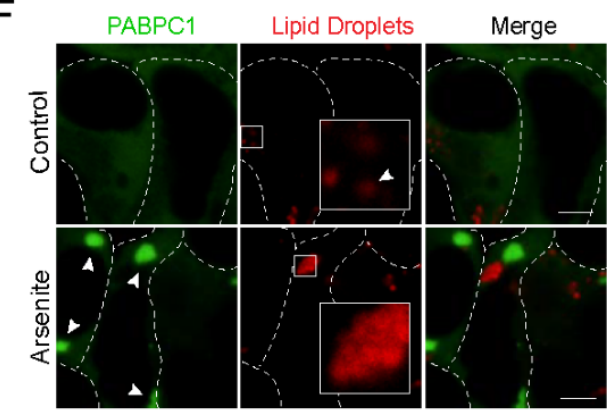

G

E
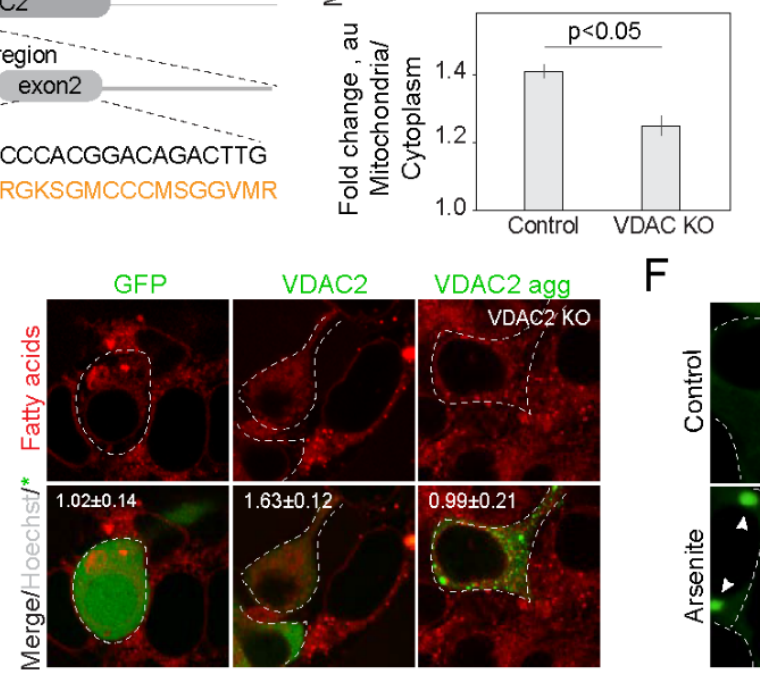

D
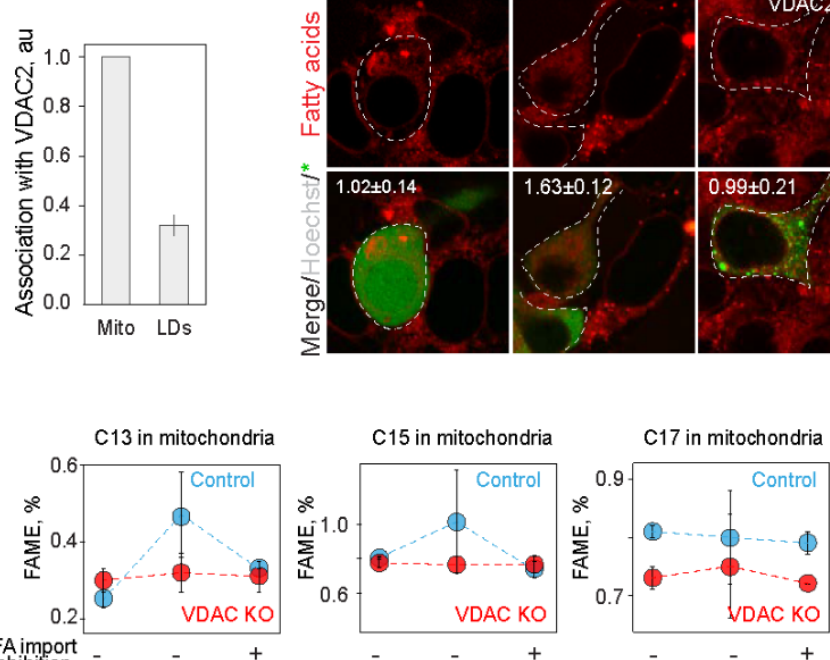

inhibition -

0 hours 4 hours 4 hours

$0 \overline{\text { hours } 4 \text { hours } 4 \text { hours }}$

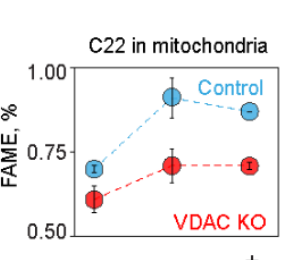

0 hours 4 hours 4 hours

$\mathrm{H}$

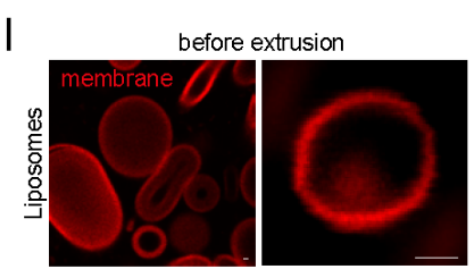

after extrusion
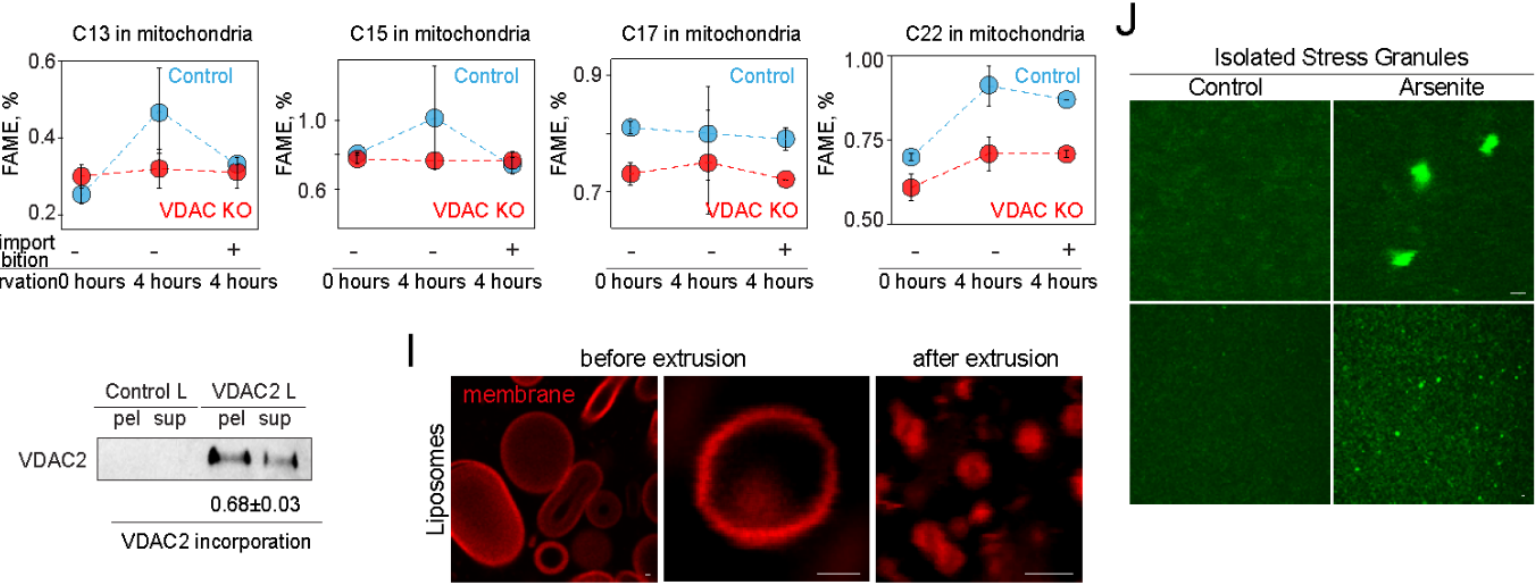


\section{Figure S7}

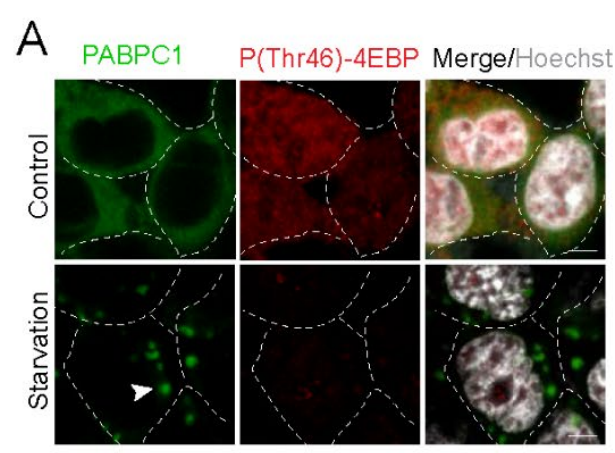

B

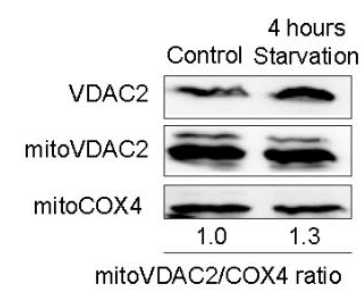

C

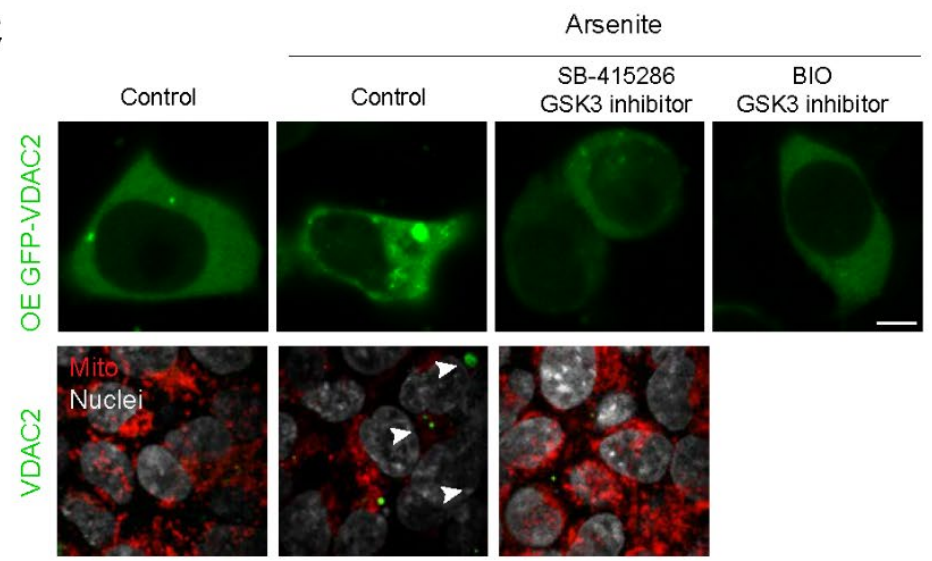

D

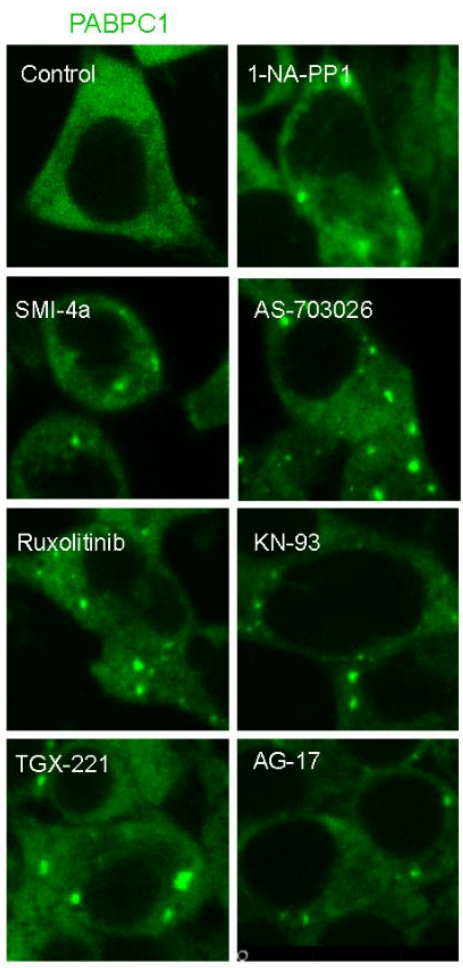




\section{Figure S8}

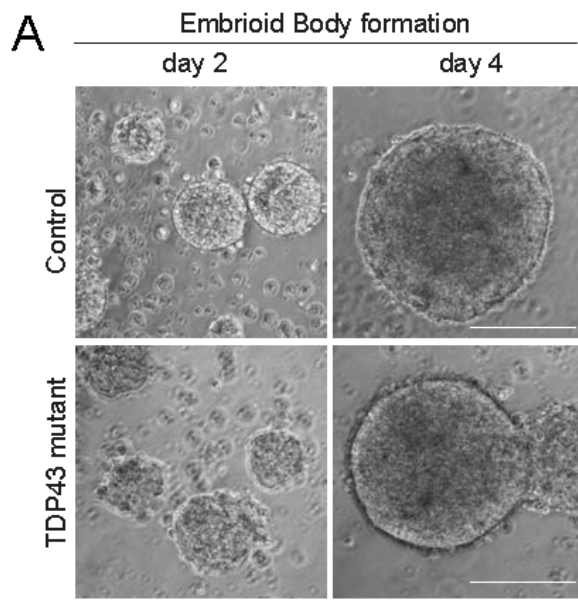

B
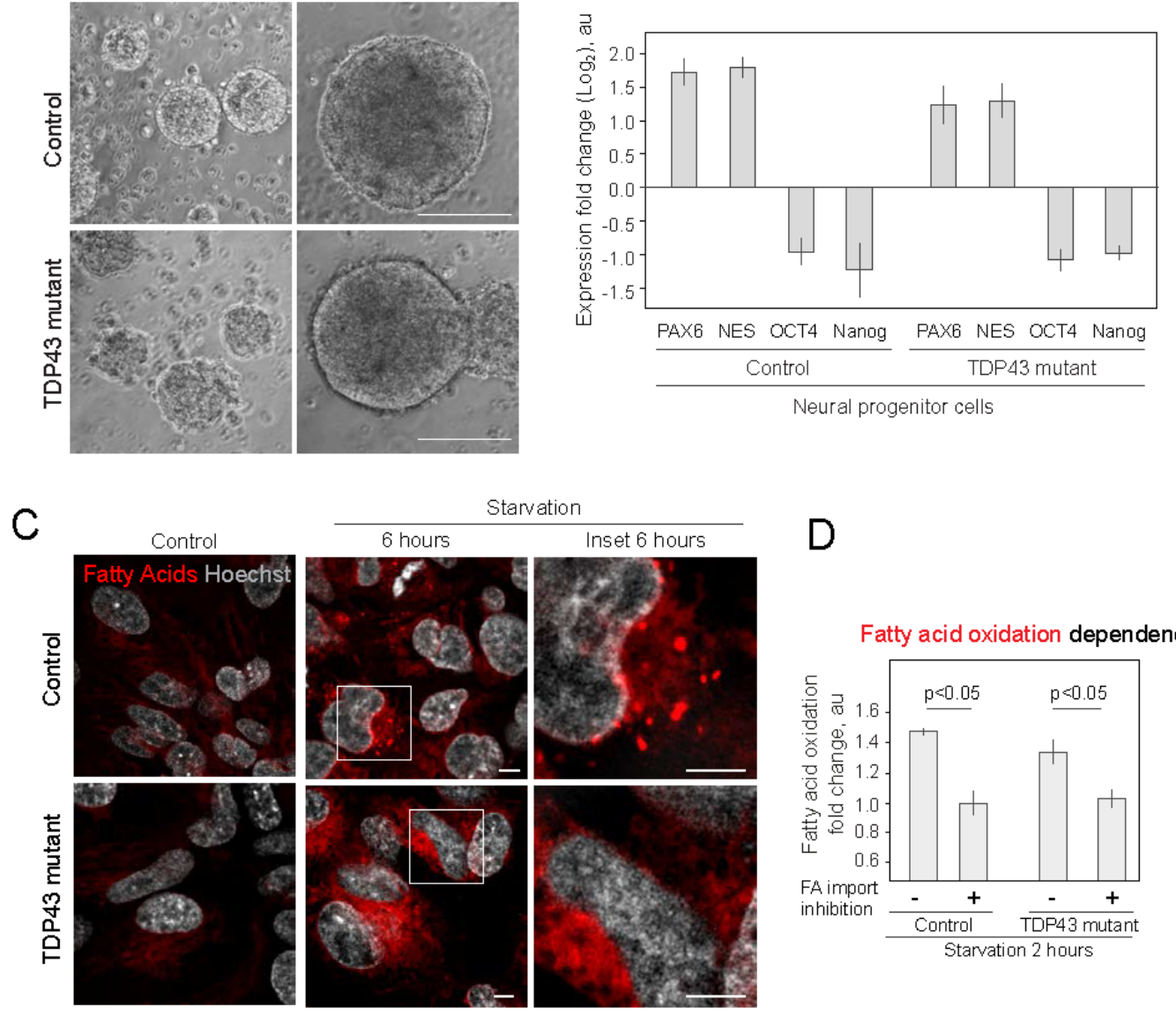


\section{References}

Amen, T., and Kaganovich, D. (2015). Dynamic droplets: the role of cytoplasmic inclusions in stress, function, and disease. Cell Mol Life Sci 72, 401-415.

Amen, T., and Kaganovich, D. (2017). Integrative modules for efficient genome engineering in yeast. Microbial Cell 4, 182-190.

Arimoto, K., Fukuda, H., Imajoh-Ohmi, S., Saito, H., and Takekawa, M. (2008). Formation of stress granules inhibits apoptosis by suppressing stress-responsive MAPK pathways. Nat Cell Biol 10, 13241332.

Asanuma, M., Kim, S.-M., Kim, H., Kim, J.-E., Park, K.S., Sung, J.-J., Kim, S.H., and Lee, K.-W. (2011). Amyotrophic Lateral Sclerosis Is Associated with Hypolipidemia at the Presymptomatic Stage in Mice. Plos One 6, e17985.

Aulas, A., and Vande Velde, C. (2015). Alterations in stress granule dynamics driven by TDP-43 and FUS: a link to pathological inclusions in ALS? Front Cell Neurosci 9, 423.

Bae, S., Park, J., and Kim, J.S. (2014). Cas-OFFinder: a fast and versatile algorithm that searches for potential off-target sites of Cas9 RNA-guided endonucleases. Bioinformatics 30, 1473-1475.

Barber, S.C., and Shaw, P.J. (2010). Oxidative stress in ALS: Key role in motor neuron injury and therapeutic target. Free Radical Biology and Medicine 48, 629-641.

Benador, I.Y., Veliova, M., Liesa, M., and Shirihai, O.S. (2019). Mitochondria Bound to Lipid Droplets: Where Mitochondrial Dynamics Regulate Lipid Storage and Utilization. Cell Metab 29, 827-835.

Brachmann, C.B., Davies, A., Cost, G.J., Caputo, E., Li, J.C., Hieter, P., and Boeke, J.D. (1998). Designer deletion strains derived from Saccharomyces cerevisiae S288C: a useful set of strains and plasmids for PCR-mediated gene disruption and other applications. Yeast 14, 115-132.

Buchan, J.R., Yoon, J.H., and Parker, R. (2011). Stress-specific composition, assembly and kinetics of stress granules in Saccharomyces cerevisiae. J Cell Sci 124, 228-239.

Chin, H.S., Li, M.X., Tan, I.K.L., Ninnis, R.L., Reljic, B., Scicluna, K., Dagley, L.F., Sandow, J.J., Kelly, G.L., Samson, A.L., et al. (2018). VDAC2 enables BAX to mediate apoptosis and limit tumor development. Nature Communications 9.

Cioni, J.M., Lin, J.Q., Holtermann, A.V., Koppers, M., Jakobs, M.A.H., Azizi, A., Turner-Bridger, B., Shigeoka, T., Franze, K., Harris, W.A., et al. (2019). Late Endosomes Act as mRNA Translation Platforms and Sustain Mitochondria in Axons. Cell 176, 56-72 e15.

Cleveland, D.W., and Rothstein, J.D. (2001). From Charcot to Lou Gehrig: deciphering selective motor neuron death in ALS. Nat Rev Neurosci 2, 806-819.

Cong, L., Ran, F.A., Cox, D., Lin, S., Barretto, R., Habib, N., Hsu, P.D., Wu, X., Jiang, W., Marraffini, L.A., et al. (2013). Multiplex genome engineering using CRISPR/Cas systems. Science 339, 819-823.

Cortassa, S., Sollott, S.J., and Aon, M.A. (2017). Mitochondrial respiration and ROS emission during betaoxidation in the heart: An experimental-computational study. PLoS Comput Biol 13, e1005588.

Cox, J., Hein, M.Y., Luber, C.A., Paron, I., Nagaraj, N., and Mann, M. (2014). Accurate proteome-wide label-free quantification by delayed normalization and maximal peptide ratio extraction, termed MaxLFQ. Mol Cell Proteomics 13, 2513-2526. 
Cox, J., and Mann, M. (2008). MaxQuant enables high peptide identification rates, individualized p.p.b.range mass accuracies and proteome-wide protein quantification. Nat Biotechnol 26, 1367-1372.

Das, S., Wong, R., Rajapakse, N., Murphy, E., and Steenbergen, C. (2008). Glycogen synthase kinase 3 inhibition slows mitochondrial adenine nucleotide transport and regulates voltage-dependent anion channel phosphorylation. Circ Res 103, 983-991.

Dewey, C.M., Cenik, B., Sephton, C.F., Johnson, B.A., Herz, J., and Yu, G. (2012). TDP-43 aggregation in neurodegeneration: Are stress granules the key? Brain Research 1462, 16-25.

Douglas, D.N., Pu, C.H., Lewis, J.T., Bhat, R., Anwar-Mohamed, A., Logan, M., Lund, G., Addison, W.R., Lehner, R., and Kneteman, N.M. (2016). Oxidative Stress Attenuates Lipid Synthesis and Increases Mitochondrial Fatty Acid Oxidation in Hepatoma Cells Infected with Hepatitis C Virus. J Biol Chem 291, 1974-1990.

Dupuis, L., and Loeffler, J.P. (2009). Neuromuscular junction destruction during amyotrophic lateral sclerosis: insights from transgenic models. Curr Opin Pharmacol 9, 341-346.

Eliyahu, E., Pnueli, L., Melamed, D., Scherrer, T., Gerber, A.P., Pines, O., Rapaport, D., and Arava, Y. (2010). Tom 20 mediates localization of mRNAs to mitochondria in a translation-dependent manner. Mol Cell Biol 30, 284-294.

Emara, M.M., Fujimura, K., Sciaranghella, D., Ivanova, V., Ivanov, P., and Anderson, P. (2012). Hydrogen peroxide induces stress granule formation independent of eIF2 $\alpha$ phosphorylation. Biochemical and Biophysical Research Communications 423, 763-769.

Fang, D., and Maldonado, E.N. (2018). VDAC Regulation: A Mitochondrial Target to Stop Cell

Proliferation. 138, 41-69.

Fatscher, T., Boehm, V., Weiche, B., and Gehring, N.H. (2014). The interaction of cytoplasmic poly(A)binding protein with eukaryotic initiation factor $4 \mathrm{G}$ suppresses nonsense-mediated mRNA decay. RNA 20, 1579-1592.

Folch, J., Lees, M., and Sloane Stanley, G.H. (1957). A simple method for the isolation and purification of total lipides from animal tissues. J Biol Chem 226, 497-509.

Fournier, M.J., Coudert, L., Mellaoui, S., Adjibade, P., Gareau, C., Cote, M.F., Sonenberg, N., Gaudreault, R.C., and Mazroui, R. (2013). Inactivation of the mTORC1-eukaryotic translation initiation factor 4E pathway alters stress granule formation. Mol Cell Biol 33, 2285-2301.

Freibaum, B.D., Chitta, R.K., High, A.A., and Taylor, J.P. (2010). Global Analysis of TDP-43 Interacting Proteins Reveals Strong Association with RNA Splicing and Translation Machinery. Journal of Proteome Research 9, 1104-1120.

Frings, C.S., Fendley, T.W., Dunn, R.T., and Queen, C.A. (1972). Improved determination of total serum lipids by the sulfo-phospho-vanillin reaction. Clin Chem 18, 673-674.

Fukada, K., Zhang, F., Vien, A., Cashman, N.R., and Zhu, H. (2004). Mitochondrial Proteomic Analysis of a Cell Line Model of Familial Amyotrophic Lateral Sclerosis. Molecular \& Cellular Proteomics 3, 12111223.

Gietz, R.D., Schiestl, R.H., Willems, A.R., and Woods, R.A. (1995). Studies on the Transformation of Intact Yeast-Cells by the Liac/S-DNA/Peg Procedure. Yeast 11, 355-360.

Gordon, D., Dafinca, R., Scaber, J., Alegre-Abarrategui, J., Farrimond, L., Scott, C., Biggs, D., Kent, L., Oliver, P.L., Davies, B., et al. (2019). Single-copy expression of an amyotrophic lateral sclerosis-linked 
TDP-43 mutation (M337V) in BAC transgenic mice leads to altered stress granule dynamics and progressive motor dysfunction. Neurobiol Dis 121, 148-162.

Gottschling, D.E., and Nystrom, T. (2017). The Upsides and Downsides of Organelle Interconnectivity. Cell 169, 24-34.

Guo, Y., Cordes, K.R., Farese, R.V., and Walther, T.C. (2009). Lipid droplets at a glance. Journal of Cell Science $122,749-752$.

Haimovich, G., Cohen-Zontag, O., and Gerst, J.E. (2016). A role for mRNA trafficking and localized translation in peroxisome biogenesis and function? Biochimica et Biophysica Acta (BBA) - Molecular Cell Research 1863, 911-921.

Hamada, T., Yako, M., Minegishi, M., Sato, M., Kamei, Y., Yanagawa, Y., Toyooka, K., Watanabe, Y., and Hara-Nishimura, I. (2018). Stress granule formation is induced by a threshold temperature rather than a temperature difference inArabidopsis. Journal of Cell Science 131, jcs216051.

He, F., Fromion, V., and Westerhoff, H.V. (2013). (Im)Perfect robustness and adaptation of metabolic networks subject to metabolic and gene-expression regulation: marrying control engineering with metabolic control analysis. BMC Syst Biol 7, 131.

Heberle, A.M., Prentzell, M.T., van Eunen, K., Bakker, B.M., Grellscheid, S.N., and Thedieck, K. (2015). Molecular mechanisms of mTOR regulation by stress. Mol Cell Oncol 2, e970489.

Heberle, A.M., Razquin Navas, P., Langelaar-Makkinje, M., Kasack, K., Sadik, A., Faessler, E., Hahn, U., Marx-Stoelting, P., Opitz, C.A., Sers, C., et al. (2019). The PI3K and MAPK/p38 pathways control stress granule assembly in a hierarchical manner. Life Sci Alliance 2.

Hermida, M.A., Dinesh Kumar, J., and Leslie, N.R. (2017). GSK3 and its interactions with the $\mathrm{PI3K} / \mathrm{AKT} / \mathrm{mTOR}$ signalling network. Adv Biol Regul 65, 5-15.

Holtta-Vuori, M., Uronen, R.L., Repakova, J., Salonen, E., Vattulainen, I., Panula, P., Li, Z., Bittman, R., and Ikonen, E. (2008). BODIPY-cholesterol: a new tool to visualize sterol trafficking in living cells and organisms. Traffic 9, 1839-1849.

Hunter, J.D. (2007). Matplotlib: A 2D graphics environment. Comput Sci Eng 9, 90-95.

Hunter, P. (2009). Robust yet flexible. In biological systems, resistance to change and innovation in the light of it go hand in hand. EMBO Rep 10, 949-952.

Isabelle, M., Gagne, J.P., Gallouzi, I.E., and Poirier, G.G. (2012). Quantitative proteomics and dynamic imaging reveal that G3BP-mediated stress granule assembly is poly(ADP-ribose)-dependent following exposure to MNNG-induced DNA alkylation. Journal of Cell Science 125, 4555-4566.

Jain, S., Wheeler, Joshua R., Walters, Robert W., Agrawal, A., Barsic, A., and Parker, R. (2016). ATPaseModulated Stress Granules Contain a Diverse Proteome and Substructure. Cell 164, 487-498.

Jevtov, I., Zacharogianni, M., van Oorschot, M.M., van Zadelhoff, G., Aguilera-Gomez, A., Vuillez, I., Braakman, I., Hafen, E., Stocker, H., and Rabouille, C. (2015). TORC2 mediates the heat stress response in Drosophila by promoting the formation of stress granules. J Cell Sci 128, 2497-2508.

Kaganovich, D. (2017). There Is an Inclusion for That: Material Properties of Protein Granules Provide a Platform for Building Diverse Cellular Functions. Trends Biochem Sci 42, 765-776.

Kaganovich, D., Kopito, R., and Frydman, J. (2008). Misfolded proteins partition between two distinct quality control compartments. Nature 454, 1088-U1036. 
Kajihara, N., Kukidome, D., Sada, K., Motoshima, H., Furukawa, N., Matsumura, T., Nishikawa, T., and Araki, E. (2017). Low glucose induces mitochondrial reactive oxygen species via fatty acid oxidation in bovine aortic endothelial cells. J Diabetes Investig 8, 750-761.

Kamelgarn, M., Chen, J., Kuang, L., Arenas, A., Zhai, J., Zhu, H., and Gal, J. (2016). Proteomic analysis of FUS interacting proteins provides insights into FUS function and its role in ALS. Biochimica et Biophysica Acta (BBA) - Molecular Basis of Disease 1862, 2004-2014.

Kedersha, N., and Anderson, P. (2007). Mammalian stress granules and processing bodies. Methods Enzymol 431, 61-81.

Kedersha, N., Ivanov, P., and Anderson, P. (2013). Stress granules and cell signaling: more than just a passing phase? Trends Biochem Sci 38, 494-506.

Kedersha, N., Stoecklin, G., Ayodele, M., Yacono, P., Lykke-Andersen, J., Fritzler, M.J., Scheuner, D., Kaufman, R.J., Golan, D.E., and Anderson, P. (2005). Stress granules and processing bodies are dynamically linked sites of mRNP remodeling. J Cell Biol 169, 871-884.

Kerner, J., and Hoppel, C. (2000). Fatty acid import into mitochondria. Biochimica et Biophysica Acta (BBA) - Molecular and Cell Biology of Lipids 1486, 1-17.

Kerppola, T.K. (2006). Design and implementation of bimolecular fluorescence complementation (BiFC) assays for the visualization of protein interactions in living cells. Nature Protocols 1, 1278-1286.

Khong, A., Matheny, T., Jain, S., Mitchell, S.F., Wheeler, J.R., and Parker, R. (2017). The Stress Granule Transcriptome Reveals Principles of mRNA Accumulation in Stress Granules. Mol Cell 68, 808-820 e805.

Khor, V.K., Ahrends, R., Lin, Y., Shen, W.J., Adams, C.M., Roseman, A.N., Cortez, Y., Teruel, M.N., Azhar, S., and Kraemer, F.B. (2014). The Proteome of Cholesteryl-Ester-Enriched Versus TriacylglycerolEnriched Lipid Droplets. Plos One 9.

Kim, H.J., Kim, N.C., Wang, Y.D., Scarborough, E.A., Moore, J., Diaz, Z., MacLea, K.S., Freibaum, B., Li, S.Q., Molliex, A., et al. (2013). Mutations in prion-like domains in hnRNPA2B1 and hnRNPA1 cause multisystem proteinopathy and ALS. Nature 495, 467-+.

Kobayashi, T., Winslow, S., Sunesson, L., Hellman, U., and Larsson, C. (2012). PKCalpha binds G3BP2 and regulates stress granule formation following cellular stress. PLoS One 7, e35820.

Lauterwasser, J., Todt, F., Zerbes, R.M., Nguyen, T.N., Craigen, W., Lazarou, M., van der Laan, M., and Edlich, F. (2016). The porin VDAC2 is the mitochondrial platform for Bax retrotranslocation. Sci Rep-Uk 6.

Lee, J.E., Cathey, P.I., Wu, H., Parker, R., and Voeltz, G.K. (2020). Endoplasmic reticulum contact sites regulate the dynamics of membraneless organelles. Science 367.

Lee, K., Kerner, J., and Hoppel, C.L. (2011). Mitochondrial carnitine palmitoyltransferase 1a (CPT1a) is part of an outer membrane fatty acid transfer complex. J Biol Chem 286, 25655-25662.

Lenzi, J., De Santis, R., de Turris, V., Morlando, M., Laneve, P., Calvo, A., Caliendo, V., Chio, A., Rosa, A., and Bozzoni, I. (2015). ALS mutant FUS proteins are recruited into stress granules in induced pluripotent stem cell-derived motoneurons. Disease Models \& Mechanisms 8, 755-766.

Li, Y.R., King, O.D., Shorter, J., and Gitler, A.D. (2013a). Stress granules as crucibles of ALS pathogenesis. J Cell Biol 201, 361-372.

Li, Y.R., King, O.D., Shorter, J., and Gitler, A.D. (2013b). Stress granules as crucibles of ALS pathogenesis. The Journal of Cell Biology 201, 361-372. 
Liao, Y.C., Fernandopulle, M.S., Wang, G., Choi, H., Hao, L., Drerup, C.M., Patel, R., Qamar, S., NixonAbell, J., Shen, Y., et al. (2019). RNA Granules Hitchhike on Lysosomes for Long-Distance Transport, Using Annexin A11 as a Molecular Tether. Cell 179, 147-164 e120.

Lin, Y., Mori, E., Kato, M., Xiang, S., Wu, L., Kwon, I., and McKnight, S.L. (2016). Toxic PR PolyDipeptides Encoded by the C9orf72 Repeat Expansion Target LC Domain Polymers. Cell 167, 789-802 e712.

Ling, S.C., Albuquerque, C.P., Han, J.S., Lagier-Tourenne, C., Tokunaga, S., Zhou, H., and Cleveland, D.W. (2010). ALS-associated mutations in TDP-43 increase its stability and promote TDP-43 complexes with FUS/TLS. Proceedings of the National Academy of Sciences 107, 13318-13323.

Listenberger, L.L., Han, X., Lewis, S.E., Cases, S., Farese, R.V., Jr., Ory, D.S., and Schaffer, J.E. (2003). Triglyceride accumulation protects against fatty acid-induced lipotoxicity. Proc Natl Acad Sci U S A 100, 3077-3082.

Liu, Y., Wang, T., Ji, Y.J., Johnson, K., Liu, H., Johnson, K., Bailey, S., Suk, Y., Lu, Y.N., Liu, M., et al. (2018). A C9orf72-CARM1 axis regulates lipid metabolism under glucose starvation-induced nutrient stress. Genes Dev 32, 1380-1397.

Livak, K.J., and Schmittgen, T.D. (2001). Analysis of relative gene expression data using real-time quantitative PCR and the 2(-Delta Delta C(T)) Method. Methods 25, 402-408.

Lu, X., Drocco, J., and Wieschaus, E.F. (2010). Cell cycle regulation via inter-nuclear communication during the early embryonic development of Drosophila melanogaster. Cell Cycle 9, 2908-2910.

Mackenzie, I.R., Nicholson, A.M., Sarkar, M., Messing, J., Purice, M.D., Pottier, C., Annu, K., Baker, M., Perkerson, R.B., Kurti, A., et al. (2017). TIA1 Mutations in Amyotrophic Lateral Sclerosis and Frontotemporal Dementia Promote Phase Separation and Alter Stress Granule Dynamics. Neuron 95, 808816 e809.

Maharana, S., Wang, J., Papadopoulos, D.K., Richter, D., Pozniakovsky, A., Poser, I., Bickle, M., Rizk, S., Guillen-Boixet, J., Franzmann, T.M., et al. (2018). RNA buffers the phase separation behavior of prionlike RNA binding proteins. Science 360, 918-921.

Mann, J.R., Gleixner, A.M., Mauna, J.C., Gomes, E., DeChellis-Marks, M.R., Needham, P.G., Copley, K.E., Hurtle, B., Portz, B., Pyles, N.J., et al. (2019). RNA Binding Antagonizes Neurotoxic Phase Transitions of TDP-43. Neuron 102, 321-338 e328.

Martel, C., Allouche, M., Esposti, D.D., Fanelli, E., Boursier, C., Henry, C., Chopineau, J., Calamita, G., Kroemer, G., Lemoine, A., et al. (2013). Glycogen synthase kinase 3-mediated voltage-dependent anion channel phosphorylation controls outer mitochondrial membrane permeability during lipid accumulation. Hepatology 57, 93-102.

Mateju, D., Franzmann, T.M., Patel, A., Kopach, A., Boczek, E.E., Maharana, S., Lee, H.O., Carra, S., Hyman, A.A., and Alberti, S. (2017). An aberrant phase transition of stress granules triggered by misfolded protein and prevented by chaperone function. EMBO J 36, 1669-1687.

Maurya, S.R., and Mahalakshmi, R. (2016). VDAC-2: Mitochondrial outer membrane regulator masquerading as a channel? The FEBS Journal 283, 1831-1836.

McGurk, L., Gomes, E., Guo, L., Mojsilovic-Petrovic, J., Tran, V., Kalb, R.G., Shorter, J., and Bonini, N.M. (2018). Poly(ADP-Ribose) Prevents Pathological Phase Separation of TDP-43 by Promoting Liquid Demixing and Stress Granule Localization. Mol Cell 71, 703-717 e709. 
Mihaylova, M.M., Cheng, C.W., Cao, A.Q., Tripathi, S., Mana, M.D., Bauer-Rowe, K.E., Abu-Remaileh, M., Clavain, L., Erdemir, A., Lewis, C.A., et al. (2018). Fasting Activates Fatty Acid Oxidation to Enhance Intestinal Stem Cell Function during Homeostasis and Aging. Cell Stem Cell 22, 769-778 e764.

Moldavski, O., Amen, T., Levin-Zaidman, S., Eisenstein, M., Rogachev, I., Brandis, A., Kaganovich, D., and Schuldiner, M. (2015). Lipid Droplets Are Essential for Efficient Clearance of Cytosolic Inclusion Bodies. Dev Cell 33, 603-610.

Moutaoufik, M.T., El Fatimy, R., Nassour, H., Gareau, C., Lang, J., Tanguay, R.M., Mazroui, R., and Khandjian, E.W. (2014). UVC-induced stress granules in mammalian cells. PLoS One 9, e112742.

Nagai, T., Ibata, K., Park, E.S., Kubota, M., Mikoshiba, K., and Miyawaki, A. (2002). A variant of yellow fluorescent protein with fast and efficient maturation for cell-biological applications. Nat Biotechnol 20, $87-90$.

Naghdi, S., and Hajnóczky, G. (2016). VDAC2-specific cellular functions and the underlying structure. Biochimica et Biophysica Acta (BBA) - Molecular Cell Research 1863, 2503-2514.

Nguyen, T.B., Louie, S.M., Daniele, J.R., Tran, Q., Dillin, A., Zoncu, R., Nomura, D.K., and Olzmann, J.A. (2017). DGAT1-Dependent Lipid Droplet Biogenesis Protects Mitochondrial Function during Starvation-Induced Autophagy. Dev Cell 42, 9-21 e25.

Olzmann, J.A., and Carvalho, P. (2018). Dynamics and functions of lipid droplets. Nature Reviews Molecular Cell Biology 20, 137-155.

Orru, S., Coni, P., Floris, A., Littera, R., Carcassi, C., Sogos, V., and Brancia, C. (2016). Reduced stress granule formation and cell death in fibroblasts with the A382T mutation of TARDBP gene: evidence for loss of TDP-43 nuclear function. Hum Mol Genet 25, 4473-4483.

Panas, M.D., Ivanov, P., and Anderson, P. (2016). Mechanistic insights into mammalian stress granule dynamics. J Cell Biol 215, 313-323.

Patel, A., Lee, H.O., Jawerth, L., Maharana, S., Jahnel, M., Hein, M.Y., Stoynov, S., Mahamid, J., Saha, S., Franzmann, T.M., et al. (2015). A Liquid-to-Solid Phase Transition of the ALS Protein FUS Accelerated by Disease Mutation. Cell 162, 1066-1077.

Pennetta, G., and Welte, M.A. (2018). Emerging Links between Lipid Droplets and Motor Neuron Diseases. Developmental Cell 45, 427-432.

Piotrowska, J., Hansen, S.J., Park, N., Jamka, K., Sarnow, P., and Gustin, K.E. (2010). Stable formation of compositionally unique stress granules in virus-infected cells. J Virol 84, 3654-3665.

Pollari, E., Goldsteins, G., Bart, G.v., Koistinaho, J., and Giniatullin, R. (2014). The role of oxidative stress in degeneration of the neuromuscular junction in amyotrophic lateral sclerosis. Frontiers in Cellular Neuroscience 8 .

Rackham, O., and Brown, C.M. (2004). Visualization of RNA-protein interactions in living cells: FMRP and IMP1 interact on mRNAs. EMBO J 23, 3346-3355.

Ran, F.A., Hsu, P.D., Wright, J., Agarwala, V., Scott, D.A., and Zhang, F. (2013). Genome engineering using the CRISPR-Cas9 system. Nat Protoc 8, 2281-2308.

Rappsilber, J., Mann, M., and Ishihama, Y. (2007). Protocol for micro-purification, enrichment, prefractionation and storage of peptides for proteomics using StageTips. Nat Protoc 2, 1896-1906.

Reineke, L.C., Cheema, S.A., Dubrulle, J., and Neilson, J.R. (2018). Chronic starvation induces noncanonical pro-death stress granules. J Cell Sci 131. 
Rösch, K., Kwiatkowski, M., Hofmann, S., Schöbel, A., Grüttner, C., Wurlitzer, M., Schlüter, H., and Herker, E. (2016). Quantitative Lipid Droplet Proteome Analysis Identifies Annexin A3 as a Cofactor for HCV Particle Production. Cell Reports 16, 3219-3231.

Roux, K.J., Kim, D.I., Raida, M., and Burke, B. (2012). A promiscuous biotin ligase fusion protein identifies proximal and interacting proteins in mammalian cells. J Cell Biol 196, 801-810.

Sabatini, D.M. (2017). Twenty-five years of mTOR: Uncovering the link from nutrients to growth. Proc Natl Acad Sci U S A 114, 11818-11825.

Sahoo, P.K., Murawala, P., Sawale, P.T., Sahoo, M.R., Tripathi, M.M., Gaikwad, S.R., Seshadri, V., and Joseph, J. (2012). Wnt signalling antagonizes stress granule assembly through a Dishevelled-dependent mechanism. Biol Open 1, 109-119.

Schonfeld, P., and Wojtczak, L. (2008). Fatty acids as modulators of the cellular production of reactive oxygen species. Free Radic Biol Med 45, 231-241.

Sengupta, S., Peterson, T.R., Laplante, M., Oh, S., and Sabatini, D.M. (2010). mTORC1 controls fastinginduced ketogenesis and its modulation by ageing. Nature 468, 1100-1104.

Sfakianos, A.P., Mellor, L.E., Pang, Y.F., Kritsiligkou, P., Needs, H., Abou-Hamdan, H., Desaubry, L., Poulin, G.B., Ashe, M.P., and Whitmarsh, A.J. (2018). The mTOR-S6 kinase pathway promotes stress granule assembly. Cell Death Differ 25, 1766-1780.

Shao, L., Kinnally, K.W., and Mannella, C.A. (1996). Circular dichroism studies of the mitochondrial channel, VDAC, from Neurospora crassa. Biophys J 71, 778-786.

Sheff, M.A., and Thorn, K.S. (2004). Optimized cassettes for fluorescent protein tagging inSaccharomyces cerevisiae. Yeast 21,661-670.

Sherman, F. (2002). Getting started with yeast. Method Enzymol 350, 3-41.

Shoshan-Barmatz, V., De Pinto, V., Zweckstetter, M., Raviv, Z., Keinan, N., and Arbel, N. (2010). VDAC, a multi-functional mitochondrial protein regulating cell life and death. Mol Aspects Med 31, 227-285.

Sievers, F., Wilm, A., Dineen, D., Gibson, T.J., Karplus, K., Li, W., Lopez, R., McWilliam, H., Remmert, M., Soding, J., et al. (2011). Fast, scalable generation of high-quality protein multiple sequence alignments using Clustal Omega. Mol Syst Biol 7, 539.

Sinclair, C., Bommakanti, G., Gardinassi, L., Loebbermann, J., Johnson, M.J., Hakimpour, P., Hagan, T., Benitez, L., Todor, A., Machiah, D., et al. (2017). mTOR regulates metabolic adaptation of APCs in the lung and controls the outcome of allergic inflammation. Science 357, 1014-1021.

Smart, A.G., Amaral, L.A., and Ottino, J.M. (2008). Cascading failure and robustness in metabolic networks. Proc Natl Acad Sci U S A 105, 13223-13228.

Starck, S.R., Green, H.M., Alberola-Ila, J., and Roberts, R.W. (2004). A general approach to detect protein expression in vivo using fluorescent puromycin conjugates. Chem Biol 11, 999-1008.

Stelling, J., Sauer, U., Szallasi, Z., Doyle, F.J., 3rd, and Doyle, J. (2004). Robustness of cellular functions. Cell 118,675-685.

Szelechowski, M., Amoedo, N., Obre, E., Leger, C., Allard, L., Bonneu, M., Claverol, S., Lacombe, D., Oliet, S., Chevallier, S., et al. (2018). Metabolic Reprogramming in Amyotrophic Lateral Sclerosis. Sci Rep-Uk 8. 
Szklarczyk, D., Franceschini, A., Wyder, S., Forslund, K., Heller, D., Huerta-Cepas, J., Simonovic, M., Roth, A., Santos, A., Tsafou, K.P., et al. (2015). STRING v10: protein-protein interaction networks, integrated over the tree of life. Nucleic Acids Res 43, D447-452.

Szoka, F., Jr., and Papahadjopoulos, D. (1980). Comparative properties and methods of preparation of lipid vesicles (liposomes). Annu Rev Biophys Bioeng 9, 467-508.

Takahara, T., and Maeda, T. (2012). Transient sequestration of TORC1 into stress granules during heat stress. Mol Cell 47, 242-252.

Takahashi, M., Higuchi, M., Matsuki, H., Yoshita, M., Ohsawa, T., Oie, M., and Fujii, M. (2013). Stress granules inhibit apoptosis by reducing reactive oxygen species production. Mol Cell Biol 33, 815-829.

Tantama, M., Martinez-Francois, J.R., Mongeon, R., and Yellen, G. (2013). Imaging energy status in live cells with a fluorescent biosensor of the intracellular ATP-to-ADP ratio. Nat Commun 4, 2550.

Taylor, J.P., Brown, R.H., Jr., and Cleveland, D.W. (2016). Decoding ALS: from genes to mechanism. Nature 539, 197-206.

Tefera, T.W., and Borges, K. (2017). Metabolic Dysfunctions in Amyotrophic Lateral Sclerosis Pathogenesis and Potential Metabolic Treatments. Frontiers in Neuroscience 10.

Thedieck, K., Holzwarth, B., Prentzell, M.T., Boehlke, C., Klasener, K., Ruf, S., Sonntag, A.G., Maerz, L., Grellscheid, S.N., Kremmer, E., et al. (2013). Inhibition of mTORC1 by astrin and stress granules prevents apoptosis in cancer cells. Cell 154, 859-874.

Thomas, B.J., and Rothstein, R. (1989). Elevated recombination rates in transcriptionally active DNA. Cell $56,619-630$.

Tuite, M.F., Grousl, T., Ivanov, P., Malcova, I., Pompach, P., Frydlova, I., Slaba, R., Senohrabkova, L., Novakova, L., and Hasek, J. (2013). Heat Shock-Induced Accumulation of Translation Elongation and Termination Factors Precedes Assembly of Stress Granules in S. cerevisiae. PLoS ONE 8, e57083.

Tyanova, S., Temu, T., Sinitcyn, P., Carlson, A., Hein, M.Y., Geiger, T., Mann, M., and Cox, J. (2016). The Perseus computational platform for comprehensive analysis of (prote)omics data. Nat Methods 13, 731-740.

Vandoorne, T., De Bock, K., and Van Den Bosch, L. (2018). Energy metabolism in ALS: an underappreciated opportunity? Acta Neuropathologica 135, 489-509.

Varga, T., Czimmerer, Z., and Nagy, L. (2011). PPARs are a unique set of fatty acid regulated transcription factors controlling both lipid metabolism and inflammation. Biochim Biophys Acta 1812, 1007-1022.

Walker, A.K., Soo, K.Y., Sundaramoorthy, V., Parakh, S., Ma, Y., Farg, M.A., Wallace, R.H., Crouch, P.J., Turner, B.J., Horne, M.K., et al. (2013). ALS-associated TDP-43 induces endoplasmic reticulum stress, which drives cytoplasmic TDP-43 accumulation and stress granule formation. PLoS One 8, e81170.

Waterhouse, A.M., Procter, J.B., Martin, D.M., Clamp, M., and Barton, G.J. (2009). Jalview Version 2--a multiple sequence alignment editor and analysis workbench. Bioinformatics 25, 1189-1191.

Wheeler, J.R., Jain, S., Khong, A., and Parker, R. (2017). Isolation of yeast and mammalian stress granule cores. Methods 126, 12-17.

Yu, D., Baird, M.A., Allen, J.R., Howe, E.S., Klassen, M.P., Reade, A., Makhijani, K., Song, Y., Liu, S., Murthy, Z., et al. (2015). A naturally monomeric infrared fluorescent protein for protein labeling in vivo. Nat Methods 12, 763-765. 
Yu, T.Y., Raschle, T., Hiller, S., and Wagner, G. (2012). Solution NMR spectroscopic characterization of human VDAC-2 in detergent micelles and lipid bilayer nanodiscs. Biochim Biophys Acta 1818, 1562-1569.

Zhang, H.H., Lipovsky, A.I., Dibble, C.C., Sahin, M., and Manning, B.D. (2006). S6K1 regulates GSK3 under conditions of mTOR-dependent feedback inhibition of Akt. Mol Cell 24, 185-197.

Zhang, K., Daigle, J.G., Cunningham, K.M., Coyne, A.N., Ruan, K., Grima, J.C., Bowen, K.E., Wadhwa, H., Yang, P., Rigo, F., et al. (2018a). Stress Granule Assembly Disrupts Nucleocytoplasmic Transport. Cell 173, 958-971 e917.

Zhang, K., Daigle, J.G., Cunningham, K.M., Coyne, A.N., Ruan, K., Grima, J.C., Bowen, K.E., Wadhwa, H., Yang, P., Rigo, F., et al. (2018b). Stress Granule Assembly Disrupts Nucleocytoplasmic Transport. Cell 173, 958-971.e917. 


\section{Chapter 3}

\section{Stress Granules mediate communication between stress response and lipid biogenesis}

In the previous chapter we showed that SGs regulate lipid trafficking during long-term starvation. In this chapter we further consider Stress Granule regulation of lipid metabolism in human cells. Cells store excess fatty acids in lipid droplets. By channeling lipids away from mitochondrial fatty acid oxidation, SGs create a favorable environment for lipid droplet formation. Here we set to examine SG regulation of lipid droplet biogenesis. 


\section{Summary}

Metabolic regulation is a necessary component of all stress response pathways, because all different mechanisms of stress-adaptation place high ATP demands on the cell. Mechanisms that integrate diverse stress response pathways with their metabolic components are therefore of great interest, but few are known. We show that Stress Granule (SG) formation, a common adaptive response to a variety of stresses, is activated by agonists of the Peroxisome Proliferator Activated Receptor (PPAR) pathway. SGs, in turn, triage the translation of mRNA transcripts during stress such that PPAR targets are expressed while overall translation is inhibited. Our findings reveal a novel role of SGs in modulating metabolic regulation by PPAR, in particular promoting Lipid Droplet (LD) biogenesis.

\section{Introduction}

Lipid Droplets (LD) are ubiquitous lipid storage organelles that are involved in regulating energy homeostasis and membrane synthesis. Their importance to the cell has been thought to derive from the need to sequester excess fatty acids (FAs), as an energy reserve and to prevent lipotoxicity (Guo et al., 2009; Nguyen and Olzmann, 2017; Olzmann and Carvalho, 2019; Walther et al., 2017). Recently, however, novel unanticipated roles in stress response and protein folding have been proposed for LDs (Bischof et al., 2017; Li et al., 2012; Moldavski et al., 2015; Nguyen et al., 2017; Velazquez and Graef, 2016). Concurrently, a link between LD homeostasis and neurodegenerative disorders is beginning to emerge (Krahmer et al., 2013; Onal et al., 2017; Pennetta and Welte, 2018). These new findings clearly indicate that LDs have a stress response role, and make it less surprising that many stresses, including $\mathrm{pH}$ changes, oxidative stress, mitochondrial perturbations, endoplasmic reticulum stress response, autophagy activation, and mechanistic target of rapamycin complex (mTOR) kinase inhibition all lead to LD formation (Boren and Brindle, 2012; Gubern et al., 2009; Henne et al., 2018; Jin et al., 2018; Nguyen et al., 2017; Petan et al., 2018; Rambold et al., 2015). Although some of the molecular mechanisms governing LD biogenesis are beginning to emerge (Gubern et al., 2009; Nguyen et al., 2017; Rohwedder et al., 2014; VandeKopple et al., 2019; Walther et al., 2017), how these mechanisms are activated by stress signals is not fully understood. There is therefore a pressing need to 
determine the mode of communication between a stress signal, conventional stress responses, and lipid stress response.

In normal conditions cells sense excess FAs by activating Peroxisome Proliferator Activated Receptor (PPAR) mediated transcription, which leads, among other things, to LD biogenesis and upregulation of FA oxidation (Dalen et al., 2004; Gorga et al., 2017; Kim et al., 2017; Rodriguez and Kersten, 2017; Varga et al., 2011). PPAR nuclear receptors are activated by various ligands including FAs themselves (Poulsen et al., 2012; Varga et al., 2011). The PPAR response encompasses genes involved in FA trafficking, FA binding proteins, FA oxidation, and LD structural proteins (e.g. perilipins (PLINs)) (Rakhshandehroo et al., 2010a). The PPAR family consists of 3 members: PPAR $\alpha$, PPAR $\delta$ (also called PPAR $\beta$ ), and PPAR $\gamma$ (Poulsen et al., 2012). PPAR $\alpha$ ensures energy availability during fasting and starvation by upregulating lipid storage and FA oxidation (Contreras et al., 2013). Interestingly, PPAR activation antagonizes mTOR during fasting, leading to its inhibition (Sengupta et al., 2010). mTOR is a general regulator of translation and its inhibition leads to translation downregulation (Sabatini, 2017). It is therefore perplexing that the PPAR-regulated transcription over-rides the global translation inactivation that results from mTOR inhibition. An important piece of the puzzle seems to be the activation of Stress Granule (SG) formation by stress-induced mTOR inhibition (Fournier et al., 2013; Jevtov et al., 2015; Sfakianos et al., 2018; Thedieck et al., 2013). SGs are multifunctional membraneless organelles, with an important role in managing translation during stress, including recruitment of mTOR (Kedersha et al., 2005; Sfakianos et al., 2018; Takahara and Maeda, 2012). SGs appear during various stresses that curiously overlap with those that induce LD formation (Buchan and Parker, 2009; Buchan et al., 2011; Jevtov et al., 2015). Interestingly, SG proteins are associated with Lipid Droplets during Hepatitis C infection (Ariumi et al., 2011), and SG component DDX3 is directly involved in lipid metabolism (Shih et al., 2012; Tsai et al., 2017). In our recent work we showed that SG formation leads to the accumulation of FAs through clustering of VDAC2 mitochondrial channel (Amen and Kaganovich, in review). Thus, there is a mechanistic connection between SG formation and LD biogenesis, both of which can result from mTOR inactivation (Nguyen et al., 2017; Sfakianos et al., 2018).

Here we demonstrate a positive feedback relationship between SG formation and the PPAR translational response. We show that PPAR activation leads to the formation of SGs and that rather 
than be inhibited by SG formation, PPAR-mediated translation is promoted through the exclusion of PPAR-target transcripts from SGs. Our data identify several novel compounds that simultaneously trigger SG formation and FA accumulation in LDs. Overall, our study provides evidence that SGs promote LD biogenesis.

\section{Results:}

\section{Small molecule inducers of Lipid Droplets also trigger Stress Granule formation}

We set to explore the role of SGs in promoting LD biogenesis. Using an endogenously tagged PABPC1 cell line we screened 136 small molecule inhibitors (including kinase inhibitors, and fatty acid synthase (FASN) inhibitors) for the ability to induce LD formation, also scoring SG assembly (Figure 1A, S1). 38 molecules induced PABPC1 positive inclusion formation (Figure 1C). Next, we analyzed the upregulation of LD by calculating the accumulation of fluorescent FAs in the samples that formed SGs and in samples that didn't result in SG accumulation (Figure 1C). SG forming cells significantly upregulated LDs, while the rest of the treated cells were not different form an untreated control (Figure 1B-C). Thus, SG formation is a reliable predictor of LD biogenesis. We next examined one of the hits, a known LD inducing drug and potent FASN inhibitor and anti-cancer reagent, fasnall (Alwarawrah et al., 2016). We confirmed that PABPC1positive inclusions formed by fasnall are SGs by immunofluorescence and proteome analysis (Figure1E, 2E). The timeline of SG formation was similar to that of arsenite-induced SGs (Figure 1D, S2A) and SGs formation was not limited to HEK293T cell line (Figure S2F). SG formation correlated with fatty acid accumulation in LDs (Pearson correlation coefficient $\mathrm{r}=0.84$ ) (Figure 1F, S1C). Fasnall is a selective FASN inhibitor. To understand what role FASN plays in fasnall SG formation we created FASN knockout using CRISPR/Cas9 (Figure 1G, S2D). Interestingly, fasnall induced SG formation independent of FASN availability (Figure 1H, S2E), indicating that its effect on LD accumulation maybe independent of FASN, and therefore unconnected to metabolic stress. We tested another, FASN inhibitor, C75, which failed to induce SG formation (Figure S2B).

\section{PPAR activation triggers SG formation}


In order to upregulate lipid metabolism, cells mobilize a transcriptional response promoted by the PPAR nuclear receptor (Varga et al., 2011). Using PPAR agonists is sufficient to upregulate lipid accumulation and LD biogenesis (Kim et al., 2017). Interestingly, activation of PPAR response triggers not only FA accumulation in cells, but also SG formation (Figure 2A-B) (Poulsen et al., 2012). To monitor SG formation in live cells we used the HEK293T cell line, expressing CRISPR/Cas9 PABPC1-DDR2 (Amen and Kaganovich, manuscript in review). SG formation correlates with FA accumulation during PPAR response (Pearson correlation coefficient $=0.95$ ) (Figure 2A-B). To confirm that PABPC1 inclusions are SG we used common SG markers, G3BP and TIA1 (Figure 2C). SGs are multiprotein granules which potentially consist of over a hundred different proteins. We asked whether the SG proteome in PPAR activation conditions and in fasnall treatment conditions is similar to the proteome of SGs that form in response to arsenite treatment, a common SG inducer (Figure 2D, S3A-C). This was done by using the BioID approach, which entails expressing PABPC1-BirA (R118G)-GFP (Amen and Kaganovich, manuscript in review) resulting in proximity-associated biotinylation of SG proteins (Figure S3A-C) (Roux et al., 2012). We confirmed that biotinylated proteins are present in SGs (Figure S3A, C). SGs in PPAR activation conditions have significant overlap with fasnall-induced SGs and with arsenite-induced SGs (Figure 2E, Supplementary Table 1). Additionally, gene ontology shows a similar pattern of protein distribution, indicating that SGs are similar in all conditions (Figure 2E). Next, we decided to look at the mechanism of SG formation during PPAR activation.

\section{PPAR alpha regulates SG formation}

PPAR activation inhibits mTOR kinase signaling (San et al., 2015). Significantly, mTOR is a constituent of SGs (Sfakianos et al., 2018; Takahara and Maeda, 2012). SG formation has been proposed to regulate mTOR activity by facilitating the inhibition of mTOR kinase, whereas mTOR signaling has been proposed to regulate SG formation by suppressing translation (Figure 3A) (Fournier et al., 2013; Sfakianos et al., 2018; Takahara and Maeda, 2012; Thedieck et al., 2013).

The decline of 4EBP phosphorylation during the inhibition of mTOR nutrient sensing pathway inhibits translation (Figure 3A) and triggers SG formation, while also activating the PPAR response (Gingras et al., 1999; Sengupta et al., 2010; Teleman, 2005). We confirmed that PPAR activation was sufficient to trigger mTOR inhibition (Figure 3B). Given this reciprocal 
relationship, we hypothesized that PPAR activation triggers SG formation by inhibiting mTOR kinase. To further investigate this mechanism, we examined how different PPAR variants contribute to SG formation.

PPAR nuclear receptors have several isoforms with partially overlapping targets, PPAR $\alpha$, $\operatorname{PPAR} \delta$ (also called PPAR $\beta$ ), and PPAR $\gamma$, which are differentially expressed in cells and tissues (Figure 3C-D). HEK293T and SH-SY5Y cells both express PPAR $\alpha$, while SH-SY5Y cells also express PPAR $\gamma$ (Figure 3C-D). To study the role of PPAR $\alpha$ in SG formation we constructed a partial knockout cell line using CRISPR/Cas9 (Figure 3E, F). Indeed, while a 90\% reduction of PPAR alpha resulted in reduced FA import, as expected, due to inability to upregulate PPAR response, SG formation was also markedly decreased in the absence of just one out of the three PPAR isoforms (Figure 3F). Taken together these data suggest that the PPAR transcriptional response regulates SG formation. However, it was still not clear how SG induced translation inhibition coexists with PPAR regulated translation.

\section{SG coordinate translation of PPAR induced transcripts}

PPAR-mediated transcription during starvation requires triage at the translational level, because overall translation declines during SG formation, partially due to mTOR inhibition and accumulation of pre-initiation complexes in SGs (Lu et al., 2001). We therefore asked whether PPAR-mediated transcripts escape the overall block in translation, and if so how? To do this we constructed a PPAR reporter in the endogenously tagged PABPC1-DDR2 cell line. We placed the

mCherry fluorophore under the control of the PPAR response element (PPRE) (Figure 4A) and scored mCherry expression by Western blotting and fluorescent imaging (Figure4 A-C) (Makishima et al., 2015). What these assays showed unequivocally is that despite mTOR inhibition and SG formation in the presence of PPAR activators and arsenite, PPRE remains active and mCherry driven by PPRE escapes the overall translational inhibition (Figure 4C, E). SG formation, which is also triggered by arsenite and PPAR activation, correlated with the translation of the mCherry PPRE reporter, suggesting that SGs do not inhibit the translation of PPAR targets (Figure C-E). Alternative activation of PPAR by 2-bromopalmitate (2-BP) induced SG formation as well (Figure 4D, S3D). 
We posited that SGs may promote the translation of PPAR targets by failing to sequester the resulting mRNAs. According to a recently proposed model (Khong et al., 2017), SGs preferentially retain longer mRNAs. Most of the PPAR target mRNA transcripts that we examined are predicted to be excluded from SGs based on their size and 3' UTR length (Figure 4F, Supplementary Table 2) (Khong et al., 2017; Rakhshandehroo et al., 2010b). Those targets involve FA binding proteins, and LD proteins (Figure 4F). As additional evidence of this, we visualized the SG localization of an mRNA that is predicted to be excluded from SGs (SOD1, $<2 \mathrm{~kb}$ ), and one with is above the size cutoff to be sequestered in SGs (FASN, >7kb) (Figure 4G-H). Hence, we hypothesize based on these data that SGs may play a central role in PPAR signaling by sequestering large mRNAs irrelevant for fatty acid trafficking, and allowing other mRNAs to be translated, promoting LD biogenesis (Figure 4I).

\section{Discussion}

During stress cells activate distinct protective mechanisms, such as upregulation of chaperones, synthesis of antioxidant proteins, and increase in LD formation, allowing cells to mitigate the consequences of stress and adapt to stress conditions (Lindquist, 1981; Spriggs et al., 2010). To regulate these seemingly distant phenomena, cells reconfigure their translation patterns, often completely halting bulk translation and sequestering translation initiation complexes in SG compartments (Buchan and Parker, 2009; Guil et al., 2006; Kedersha et al., 2013; Kedersha et al., 2005). SGs are also thought to serve as a signaling hubs, sequestering major kinases, like mTOR and PKC, which functions as a master switch for stress adaptation (Heberle et al., 2019; Kaganovich, 2017; Kedersha et al., 2013; Kobayashi et al., 2012; Thedieck et al., 2013). We set out to mechanistically integrate seemingly distant stress response phenomena - SG formation and LD biogenesis.

To determine whether the model of SGs directing the PPAR transcriptional response and LD formation obtains in various stress conditions, we screened a library of 136 independent inhibitors for co-occurrence of SG formation and LD biogenesis. We found several novel compounds that induce SG formation, including fasnall, which is of great interest as a metabolic modulator and cancer therapeutic (Alwarawrah et al., 2016). Strikingly, cells with SGs 
demonstrate significant upregulation of FA accumulation in LDs, as comparing to cells in conditions that did not form SGs. These data confirm that SG and LD formation are jointly an integral part of stress response. Interestingly, the SG component DDX3 has been shown to regulate lipid homeostasis (Tsai et al., 2017). Additionally, LD interactomes in infected conditions (Rösch et al., 2016) significantly overlap with those of SGs (Amen and Kaganovich, manuscript in review), indicating that there may be additional functional connections between SGs and LDs. To explore these connections, we examined LD biogenesis regulation by PPAR transcriptional response, which upregulates FA trafficking and LD biogenesis (Sengupta et al., 2010; Rakhshandehroo et al., 2010a; Varga et al., 2011. PPAR is a nuclear receptor which, when activated, cooperate with RXR, binds to PPRE and facilitate translation of lipid metabolism effectors, including multiple FA binding proteins (FABP1-3), and LDs components (PLINs) (Poulsen et al., 2012; Rakhshandehroo et al., 2010b). We found that pharmacological activation of PPAR led to SG formation and mTOR inhibition (Sengupta et al., 2010). Additionally, 90\% reduction of the PPAR alpha isoform, whose activation was shown to inhibit the mTOR kinase (Michelet et al., 2018), resulted in a reduction in SG formation. mTOR kinase has been shown to regulate SG formation (Sfakianos et al., 2018), thus we hypothesize that TOR inhibition by PPAR activation can trigger SG formation. However, there is evidence for reciprocity of the mTORPPAR interaction, hence inhibition of $\mathrm{mTOR}$ via $\mathrm{SG}$ formation results in the activation of PPAR response (Sengupta et al., 2010; Thedieck et al., 2013). We next asked whether SG formation can indeed facilitate the PPAR response.

Translational inhibition by mTOR coincides with transcriptional activation by PPAR. How does PPAR target translation evade bulk translational inhibition, which results in SG formation during mTOR inhibition (Kedersha and Anderson, 2007; Kedersha et al., 2005; Sheikh and Fornace, 1999)? Recent findings demonstrated that SGs, while sequestering mRNA and translation pre-initiation complexes, retain longer transcripts (Khong et al., 2017). Interestingly, most of the analyzed PPAR target proteins, especially FABPs and LD components have a short transcript length, which allows them to be translated after SG formation. Active translation of PPAR targets after SG formation indicates that SG formation maybe an integral part of the PPAR response. SGs facilitate PPAR response by selective retention of other mRNAs, thus promoting LD biogenesis. PPAR targets include only a few longer transcripts, like ACOX1, encoding a peroxisomal FA beta oxidation enzyme. Thus, SG formation during the PPAR response may play a role in PPAR 
response specification, allowing cells to translate longer transcripts, while inhibiting them later by retention in SGs. Given that there are physiological stress condition, like starvation and infection (Buchan et al., 2011; Reineke et al., 2018; Sengupta et al., 2010), that can result in both SG formation and PPAR response activation, it would be interesting to understand whether general cellular stress response encompasses PPAR activation as well. Additionally, the physical association of SGs and LDs is an important direction for further research (Ariumi et al., 2011).

\section{Acknowledgments}

We thank Christof Lenz, Lisa Neuenroth, and Thierry Wasselin at the UMG Core Facility Proteomics for obtaining MS data. William Breuer provided technical assistance with Mass Spectrometry sample preparation. This work was supported by the European Research Council under the European Union's Seventh Framework Programm (FP/2007-2013)/ERC-StG2013 337713 DarkSide starting grant. TA was funded by the Jerusalem Brain Community (JBC) Doctoral Fellowship during part of this project. The authors declare no conflicts of interest. 


\section{Figure Legends:}

Figrure 1. Small molecule inducers of Lipid Droplets also trigger Stress Granule formation (A-C) Inhibitor screen for fatty acid accumulation and SG formation. Cells expressing PABPC1DDR2 were seeded on a 96-well glass bottom plates and grown to 80-90\% confluency. 136 inhibitors $(100 \mu \mathrm{M})$ were added to the media for 1 hour, fatty acid dye (Bodipy-C12, Red, $1 \mu \mathrm{M}$ ) was added 30 minutes prior to the experiment. Cells were visualized by confocal microscopy; inclusion formation and FA accumulation was assessed. Quantification (B) shows fluorescence intensity in lipid droplets, mean \pm SEM for control sample (no inhibitors added), control screen samples (samples without inclusions, $n=98$ ), Stress Granule samples (samples with inclusions, $\mathrm{n}=38$ ). Representative confocal images of the samples with inclusions are shown (C), refer to Figure S1 for full screen results.

(D) Timeline of SG formation in fasnall and arsenite conditions. Cells expressing PABPC1DDR2 were treated with arsenite $(100 \mu \mathrm{M})$ or fasnall $(50 \mu \mathrm{M})$ for indicated amounts of time. Representative confocal images are shown, scale bar $5 \mu \mathrm{m}$. Arrowheads indicate SGs. Refer to figure S2C for images in control conditions.

(E) Immunofluorescence showing SG markers localization to the PABPC1-DDR2 inclusions during fasnall treatment. PABPC1-DDR2 cells were treated with fasnall $(50 \mu \mathrm{M})$ for 1 hour. Cells were fixed and stained with anti-TIA1 and anti-G3BP antibodies. Hoechst $(10 \mu \mathrm{g} / \mathrm{ml})$ was used to stain the nucleus 30 minutes prior to imaging. Confocal planes are shown, scale bar $5 \mu \mathrm{m}$.

(F) Quantification of FA (Bodipy-C12) accumulation in LDs (H). Cells expressing CRISPR/Cas9 tagged PABPC1-DDR2 were incubated with fasnall $(50 \mu \mathrm{M})$ for 90min. LD dye (Bodipy-C12, Red, $1 \mu \mathrm{M}$ ) was added 30 minutes prior to the experiment. Graph represents fluorescence intensity of bodipy tagged FAs in LDs, mean \pm SEM, $n=30$. SGs were quantified as a $\%$ of cells in the population with PABPC1 inclusions, mean $\pm \mathrm{SEM}, \mathrm{n}=30$. Refer to Figure S2C for corresponding confocal images.

(G) Confirmation of CRISPR/Cas9 knockout (KO) of FASN. Control and FASN KO clonal population were grown to $90 \%$ confluency, lysed and analyzed by western blot. Quantification shows the ratio of FASN to GAPDH loading control (Refer to Figure S2D for immunostaining). 
(H) Analysis of Stress Granule formation in control and FASN KO cells. Cells were treated with arsenite $(100 \mu \mathrm{M})$ or fasnall $(50 \mu \mathrm{M})$ for 1 hour. Then fixed and stained with anti-FASN and antiTIA antibodies (SG marker). Representative confocal planes are shown, scale bar $5 \mu \mathrm{m}$. Refer to figure S2E for quantification.

Figure 2. PPAR activation triggers Stress Granule formation

(A) SG formation timeline during PPAR activation showing FA accumulation. Cells expressing CRISPR/Cas9 tagged PABPC1-DDR2 were incubated with rosiglitazone, clofibrate, and GW501516 $(100 \mu \mathrm{M})$ for indicated amounts of time, LD dye (Bodipy-C12, $1 \mu \mathrm{M})$ was added 30 minutes prior to the imaging together with Hoechst $(10 \mu \mathrm{g} / \mathrm{ml})$. Representative confocal images are shown. Arrows indicate SGs, Scale bar $5 \mu \mathrm{m}$.

(B) Quantification of SG formation and LD accumulation during PPAR activation (refer to Figure 1A). Graph shows \% of cells with SGs in the population and LD fluorescence intensity, mean \pm SD. Pearson Correlation coefficient (r) is 0.95

(C) Immunofluorescence showing SG markers localization to the PABPC1-DDR2 inclusions during PPAR activation. PABPC1-DDR2 cells were treated with clofibrate, rosiglitazone, and GW501516 $(100 \mu \mathrm{M})$ for 1hour. Cells were fixed and stained with anti-TIA1 and anti-G3BP antibodies. Hoechst $(10 \mu \mathrm{g} / \mathrm{ml})$ was used to stain the nucleus 15 minutes prior to imaging. Confocal planes are shown, scale bar $5 \mu \mathrm{m}$.

(D) Schematic of protein identification by Mass Spectrometry. Cells expressing PABPC1 fused to promiscuous biotin ligase BirA (R118G) and GFP were subjected to Fasnall $(100 \mu \mathrm{M})$, or PPAR activators (rosiglitazone, clofibrate, GW501516, 100 $\mu \mathrm{M}$ each) with addition of biotin for 6 hours. Cells were lysed and biotinylated proteins were captured on magnetic beads, followed by on bead digestion and Mass Spectroscopy. Refer to figure S3A-C.

(E) Gene ontology (GO) analysis of SG enriched proteins grouped by conditions. Shown annotations are significantly enriched and hierarchically arranged. Analysis is performed by STRING web tool (https://string-db.org/). Venn diagram shows the overlap between interactomes in different conditions.

Figure 3. PPAR alpha regulates Stress Granule formation 
(A) Schematic of mTOR regulation of translation favoring SG formation. During normal conditions mTOR phosphorylates 4EBP allowing translation initiation. Downregulation of mTOR results in translation inhibition and Stress Granule formation.

(B) PPAR activation leads to mTOR inactivation. Cells were incubated with PPAR activators

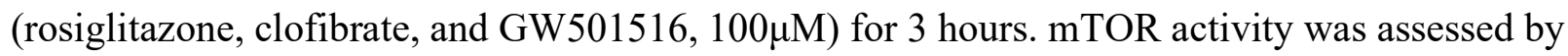
4EBP phosphorylation (Ser65) state. Ratio of phosphorylated to non-phosphorylated 4EBP is shown.

(C) PPARA isoform is prominent in SH-SY5Y cells. Cells were grown to $90 \%$ confluency. Control and arsenite (100 $\mu \mathrm{M}, 1$ hour) treated cells were lysed and analysed by western blot.

(D) PPARA isoform is prominent in HEK293 cells. Cells were grown to $90 \%$ confluency. Control and arsenite (100 $\mu \mathrm{M}, 1$ hour) treated cells were lysed and proteins were analysed by western blot.

(E) Verification of partial PPARA knockout (KO).

(F) PPARA regulates SG formation and LD accumulation. Control and PPARA knockout cells were treated with arsenite $(100 \mu \mathrm{M})$ for indicated amounts of time and fixed in $4 \%$ PFA. SG formation was analyzed with G3BP antibodies, LDs were stained with Bodipy (Green, $1 \mu \mathrm{M}$ ), Hoechst $(10 \mu \mathrm{g} / \mathrm{ml})$ was added 15 minutes prior to the imaging, confocal planes are shown, scale bar $5 \mu \mathrm{m}$. Graph represents \% of SGs in the population ( 2 bars on the left), and fluorescence intensity of FAs in the LDs ( 2 bars on the right), mean \pm SEM, $N=30$ (in each condition).

Figure 4. Stress Granules coordinate translation of PPAR induced transcripts

(A-C) PPAR response continues after SG formation. PABPC1-DDR2 cells expressing mCherry $(\mathrm{mCH})$ PPAR reporter plasmid were incubated with arsenite $(100 \mu \mathrm{M})$, cycloheximide $(10 \mu \mathrm{g} / \mathrm{ml})$,

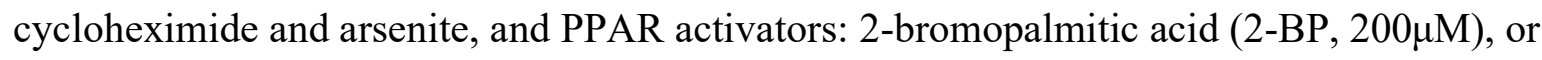

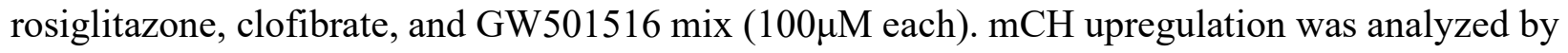
western blot (B) and by confocal microscopy (C), SG formation was analyzed by confocal microscopy (C). Arrowheads indicate SG formation. Representative confocal images are shown, scale bar $5 \mu \mathrm{m}$. 
(D) Immunofluorescence showing SG markers localization to the inclusions. PABPC1-DDR2 cells were treated with 2-BP $(200 \mu \mathrm{M})$ for 1 hour. Cells were fixed and stained with anti G3BP, and anti TIA1 antibodies. Confocal planes are shown, inlets show SGs, Scale bar $5 \mu \mathrm{m}$.

(E) Quantification (Refer to Figure 3C) shows average $\mathrm{mCH}$ fluorescence intensity during control, arsenite or 2-BP treatments. Graph shows mean $\pm \mathrm{SEM}, \mathrm{N}=30$.

(F) Distribution of PPAR target mRNA lengths. Graph shows length of 57 PPARA targets 3'UTR and full mRNA. Preference for SG localization is plotted according to Khong at al., 2017.

(G-H) Visualization of mRNA in SGs confirms increase in the retention of longer transcripts. Cells expressing PABPC1-mCherry and GFP tagged MCP were transfected either with SOD1MS2 or FASN-MS2 plasmid. SG formation was induced by arsenite (100 $\mu \mathrm{M}, 1$ hour) treatment.

(I) Graph represents fold change normalized to control for SG/cytoplasmic GFP fluorescence intensity ratio, mean $\pm \mathrm{SEM}, \mathrm{N}=30$. $(\mathrm{H})$ Confocal images showing SGs (PABPC1-mCH) and mRNA markers (GFP-MCP) distribution, scale bar $1 \mu \mathrm{m}$.

(I) Model of SG regulation of FA trafficking during PPAR response. During PPAR response cells form Stress Granules that retain longer transcripts. PPAR targets, such as PLINs and FABPs, continue being translated and promote lipid metabolism upregulating FA trafficking.

\section{Figure S1}

Inhibitor screen for fatty acid accumulation and SG formation. Cells expressing PABPC1-DDR2 were seeded on a 96-well glass bottom plates and grown to 80-90\% confluency. 136 inhibitors $(100 \mu \mathrm{M})$ were added to the media for 1 hour, fatty acid dye (Bodipy-C12, Red, $1 \mu \mathrm{M})$ was added 30 minutes prior to the experiment. Cells were visualized by confocal microscopy; inclusion formation and FA accumulation was assessed. Confocal images of all the samples are shown, refer to Figure $1 \mathrm{C}$ for zoomed in images. * - indicates fluorescence from the inhibitor.

\section{Figure S2}

(A) Control for Figure 1D: Timeline of SG formation in fasnall and arsenite conditions. Untreated cells expressing PABPC1-DDR2 were visualized. Confocal images are shown, scale bar $5 \mu \mathrm{m}$. Arrowheads indicate SGs. 
(B) Visualization of PABPC1 in fasnall, C75, and arsenite conditions. Cells expressing PABPC1-DDR2 were treated with fasnall $(50 \mu \mathrm{M})$, arsenite $(100 \mu \mathrm{M}), \mathrm{C} 75(100 \mu \mathrm{M})$ for 1 hour. Representative confocal images are shown, scale bar $5 \mu \mathrm{m}$.

(C) SG formation timeline during PPAR activation showing FA accumulation. Cells expressing CRISPR/Cas9 tagged PABPC1-DDR2 were incubated with fasnall $(50 \mu \mathrm{M})$ for indicated amounts of time, LD dye (Bodipy-C12, $1 \mu \mathrm{M}$ ) was added 30 minutes prior to the imaging together with Hoechst $(10 \mu \mathrm{g} / \mathrm{ml})$. Representative confocal images are shown. Arrows indicate SGs, Scale bar $5 \mu \mathrm{m}$. Refer to Figure 4F for quantification.

(D) Verification of FASN knockout with immunofluorescence. Cells were grown to $90 \%$ confluency fixed and FASN was visualized. Hoechst $(10 \mu \mathrm{g} / \mathrm{ml})$ was used to stain the nucleus 30 minutes prior to imaging. Representative confocal images are shown, scale bar $5 \mu \mathrm{m}$.

(E) Quantification of Stress Granule formation in fasnall and arsenite conditions in control and FASN KO cells. Refer to figure $1 \mathrm{H}$.

(F) SG formation in SHSY-5Y cells. Cells were treated with fasnall $(100 \mu \mathrm{M})$ for 1 hour and fixed. Stress Granules marker (G3BP and TIA1) were visualized using immunostaining. Representative confocal planes are shown, arrowheads indicate SGs, scale bar $5 \mu \mathrm{m}$

\section{Figure S3}

(A-C) Visualization of protein biotinylation during Stress Granule formation. Cells expressing PABPC1-BirA(R118G)-GFP were treated with fasnall $(100 \mu \mathrm{M})$ for 1 hour following addition of biotin $(100 \mu \mathrm{M})$ for 4 hours. (A) Cells were fixed in PFA and stained with streptavidin-Cy3. Confocal planes are shown, scale bar $1 \mu \mathrm{m}$. (B) Cells were lysed and biotinylated proteins were captured on magnetic beads, followed by western blot. Biotinylated proteins were visualized by streptavidin-HRP detection. (C) Graph shows fluorescence intensity of streptavidin-Cy3 (biotinylated proteins) and PABPC1-BirA(R118G)-GFP (Stress Granule) across SG inclusion (refer to S1A).

(D) SG formation in SHSY-5Y cells. Cells were treated with arsenite $(100 \mu \mathrm{M})$ or 2-BP $(200 \mu \mathrm{M})$ for 1 hour and fixed. Stress Granules marker (G3BP and TIA1) were visualized using 
immunostaining. Representative confocal planes are shown, arrowheads indicate SGs, scale bar $5 \mu \mathrm{m}$. 


\section{Figure 1}

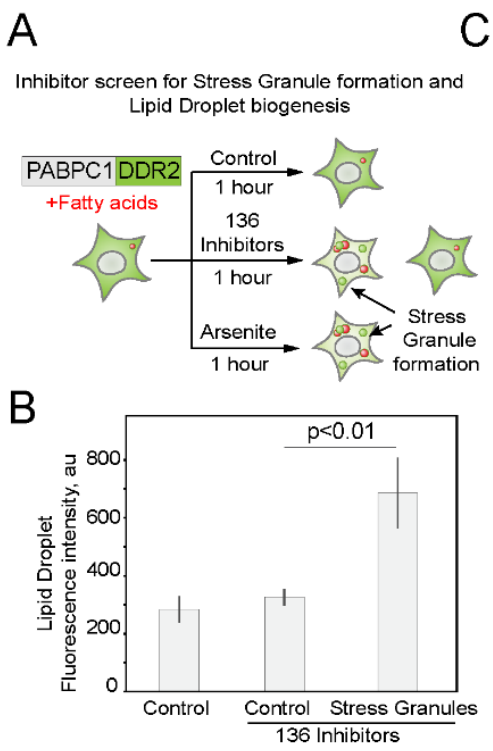

D

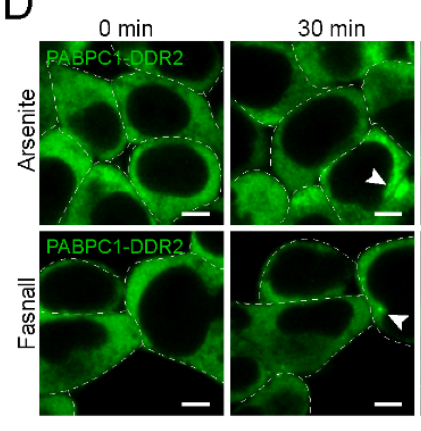

C

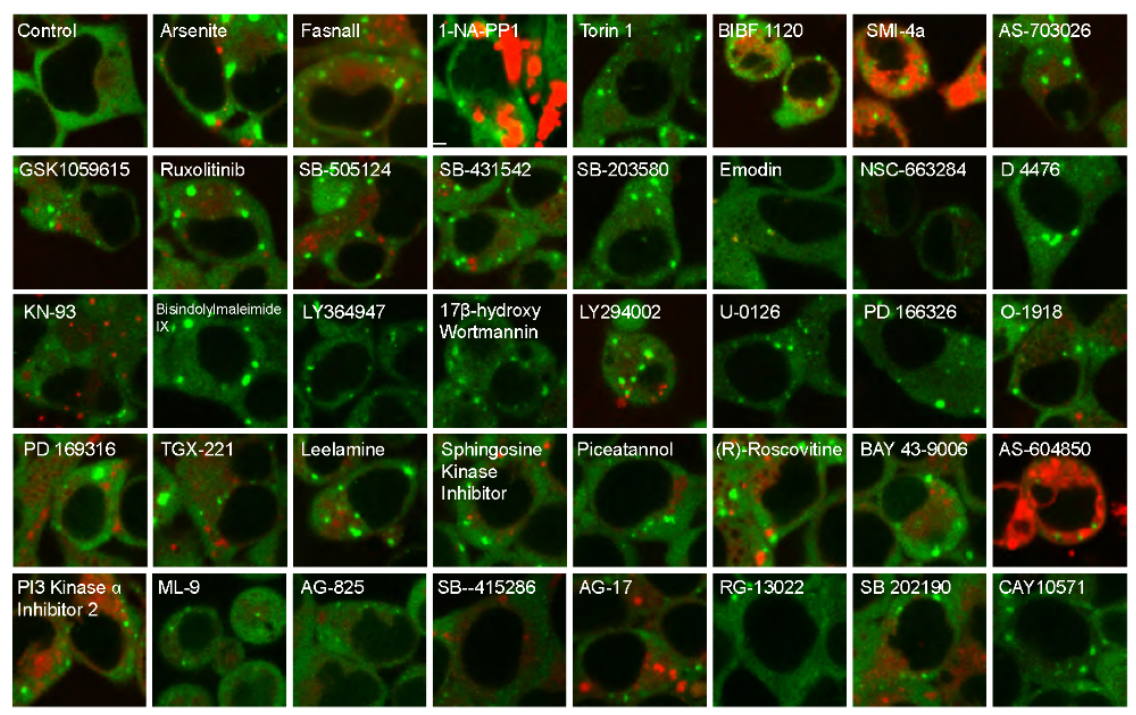

E
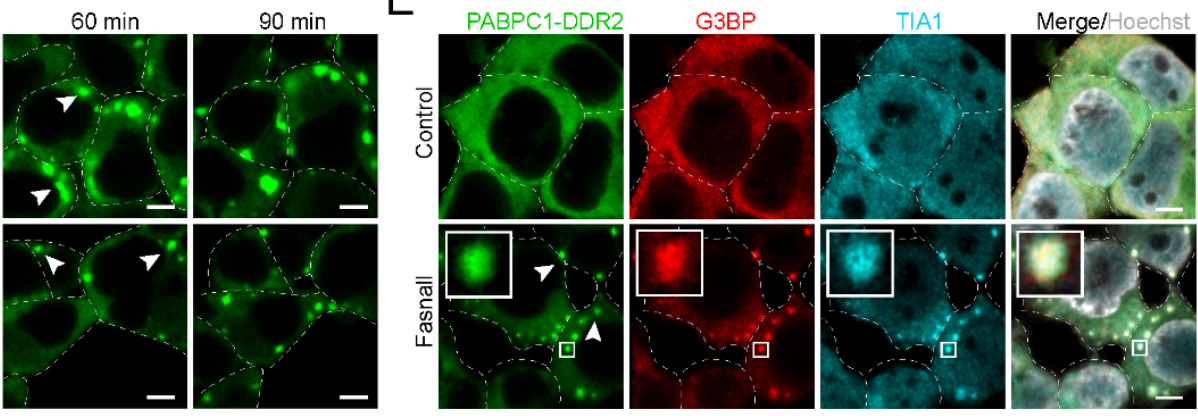

F

$\mathrm{H}$

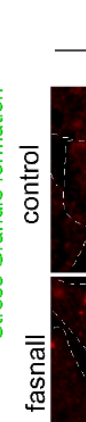

G
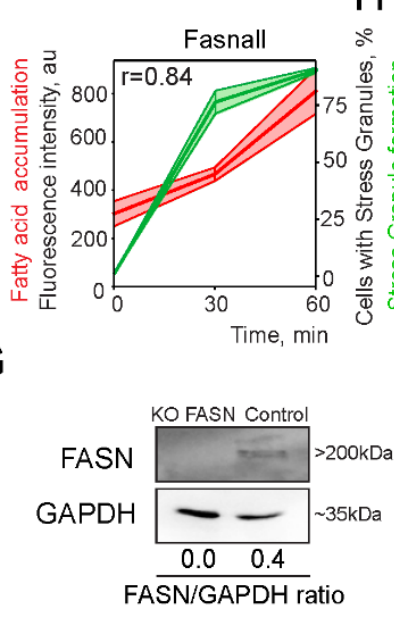

Control

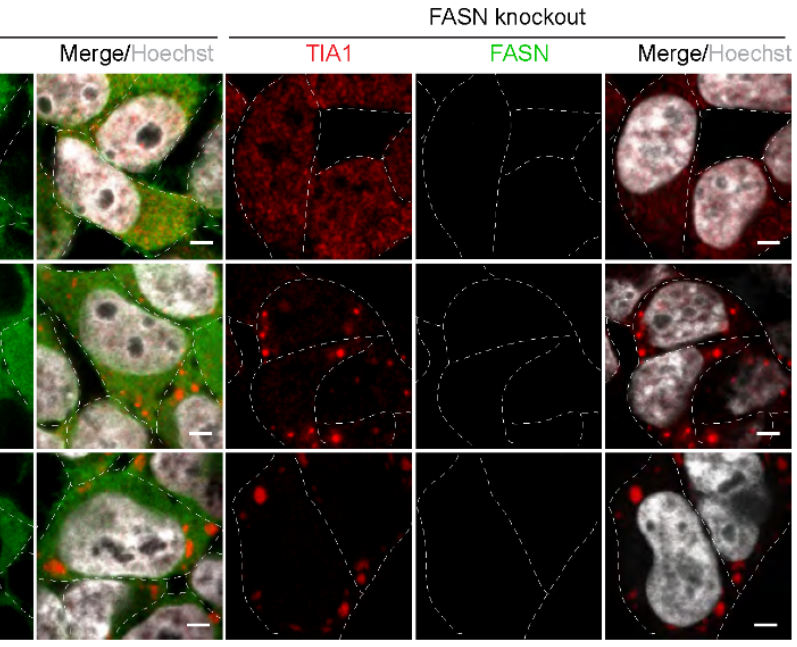




\section{Figure 2}

A

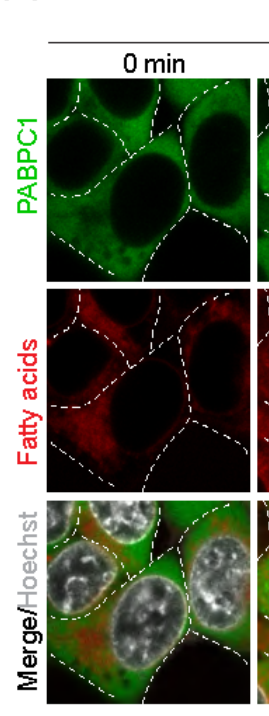

PPAR activation timecourse
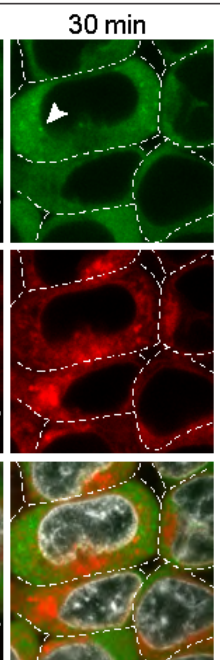

D

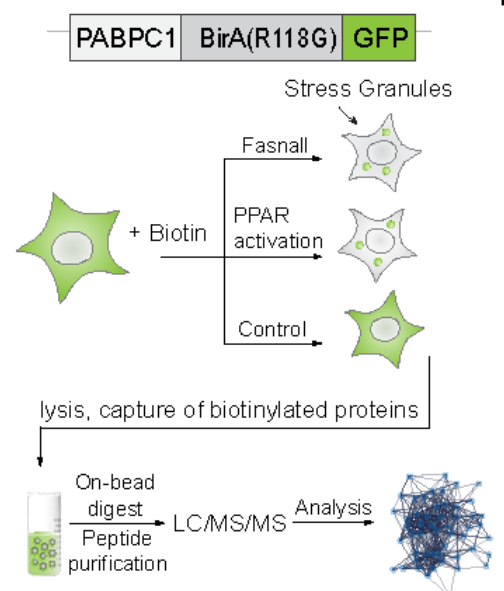

E
B
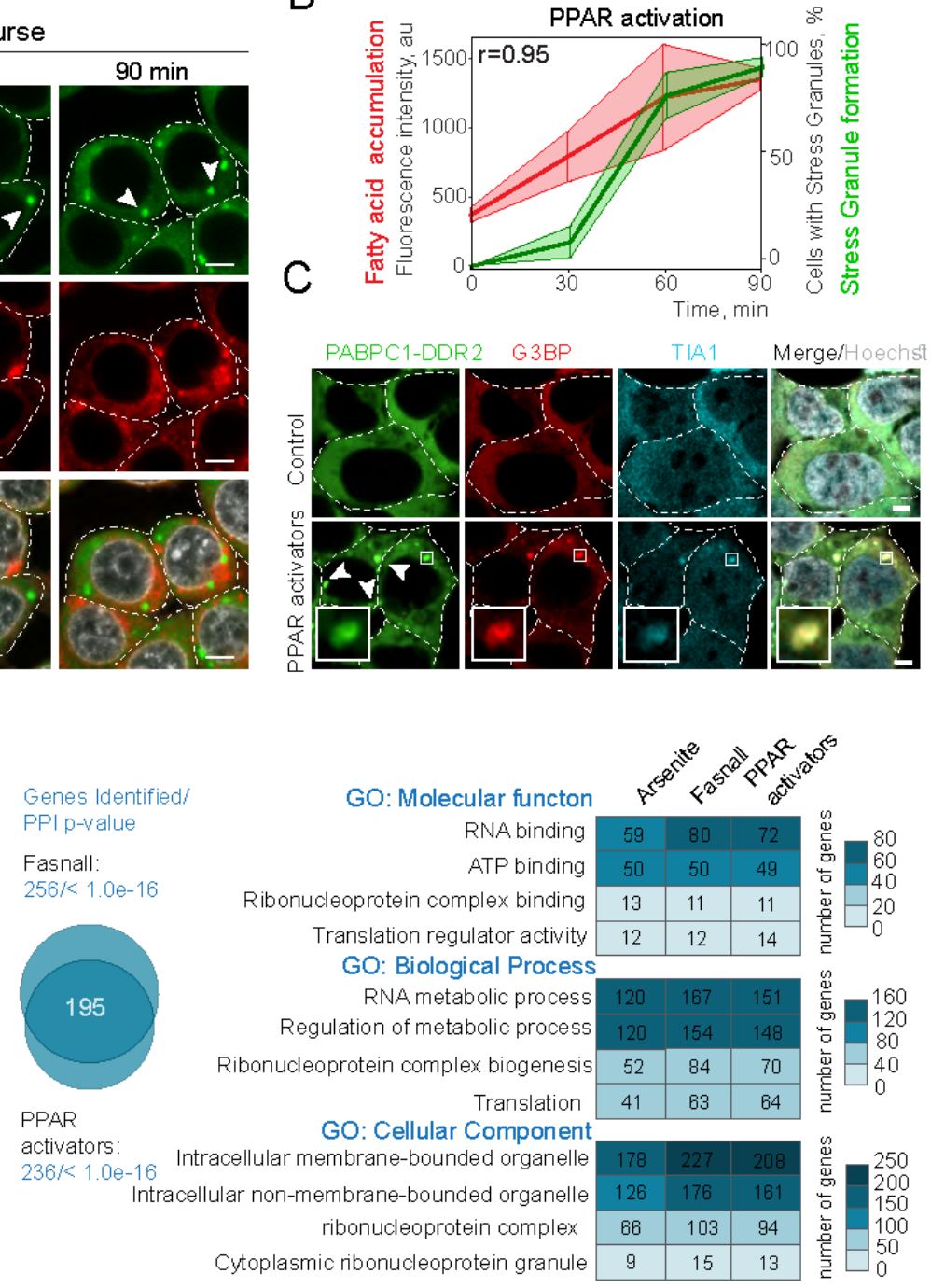


\section{Figure 3}
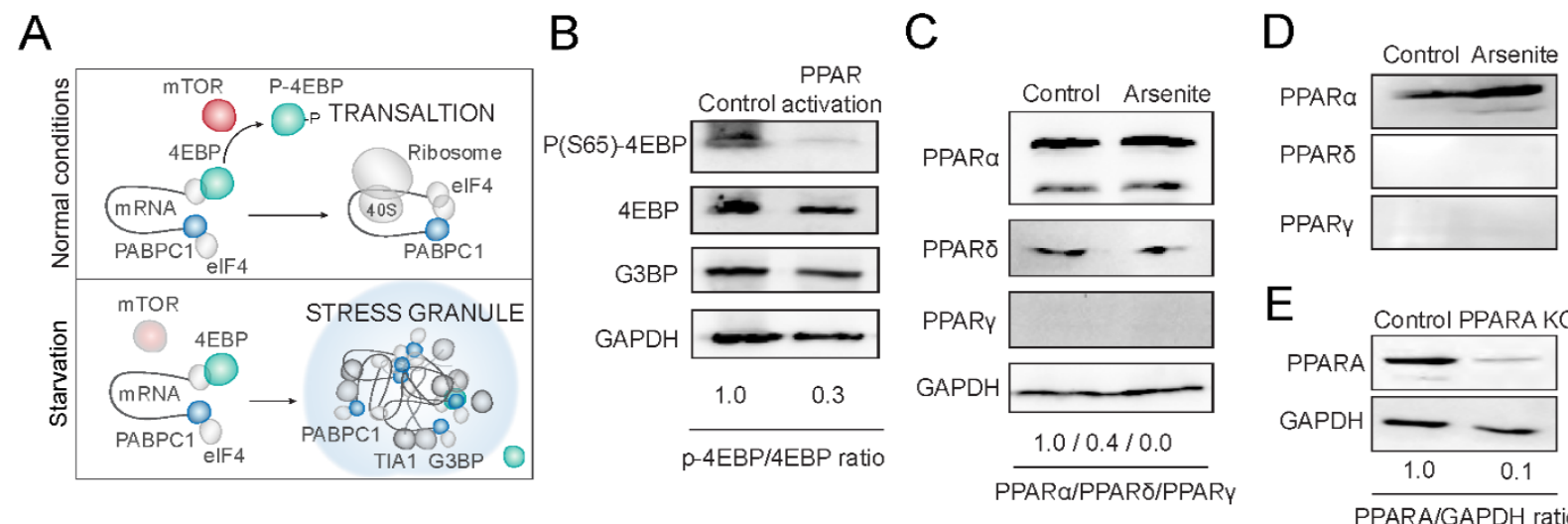

E
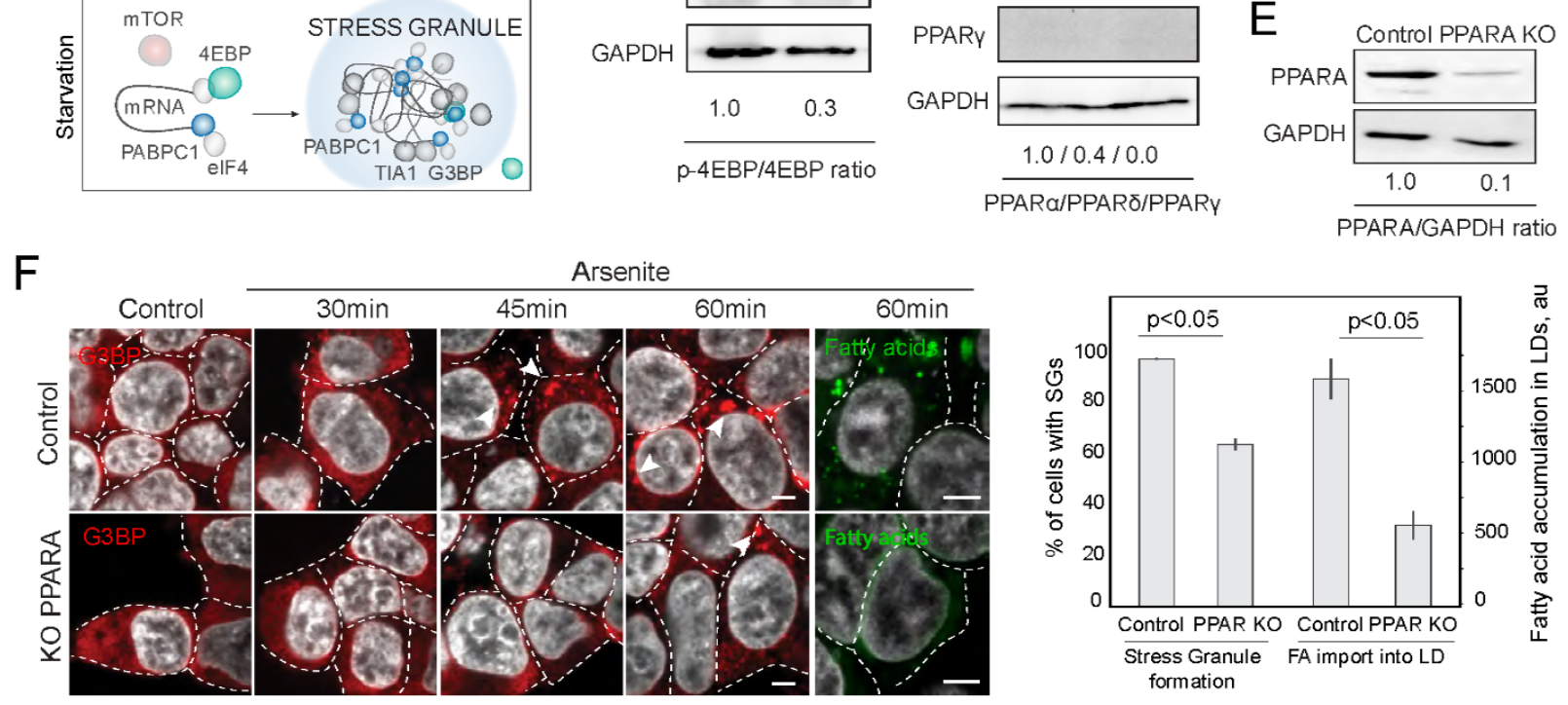


\section{Figure 4}

A

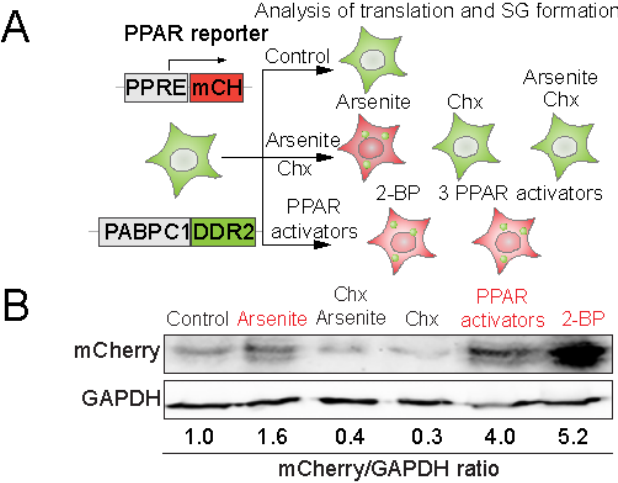

C

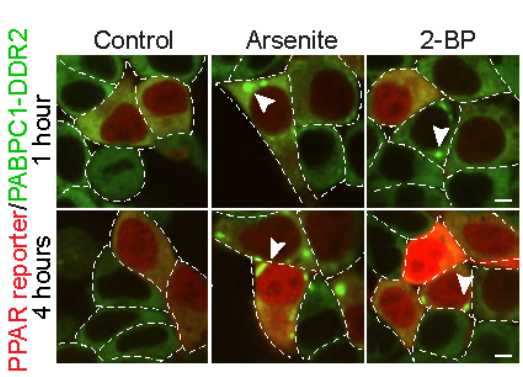

G

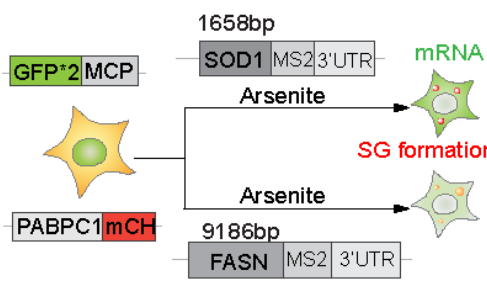

E
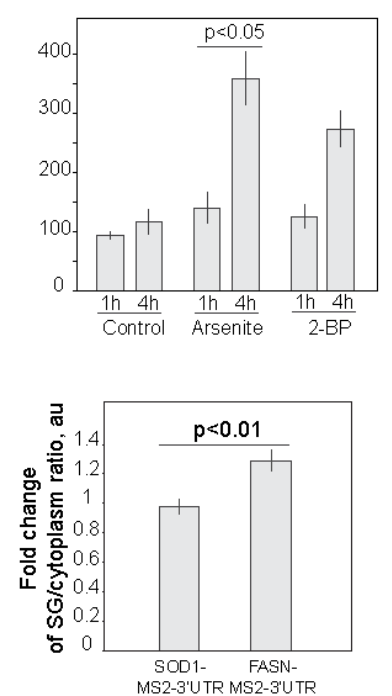

$\mathrm{H}$

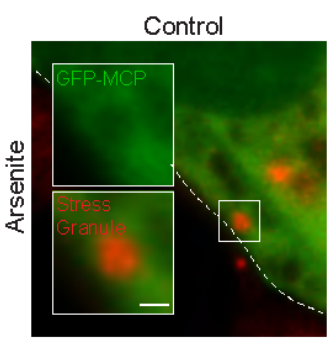

D PABPC1-DDR2
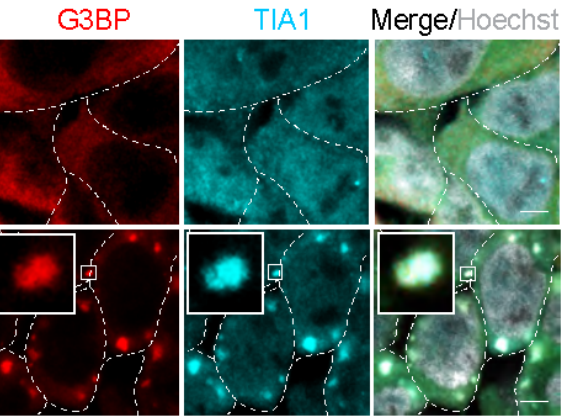

F

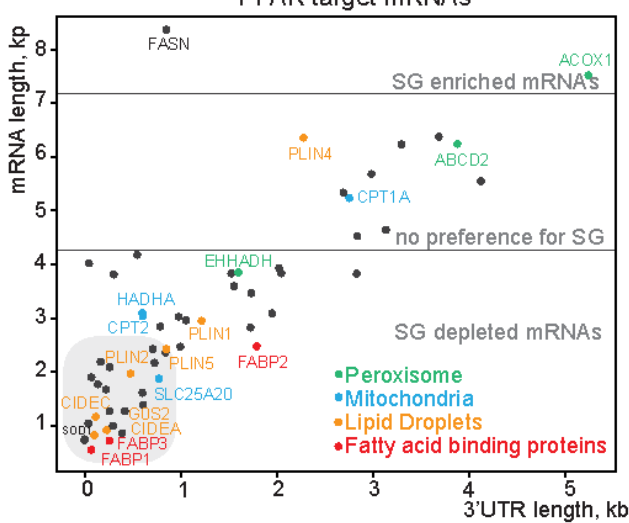

I

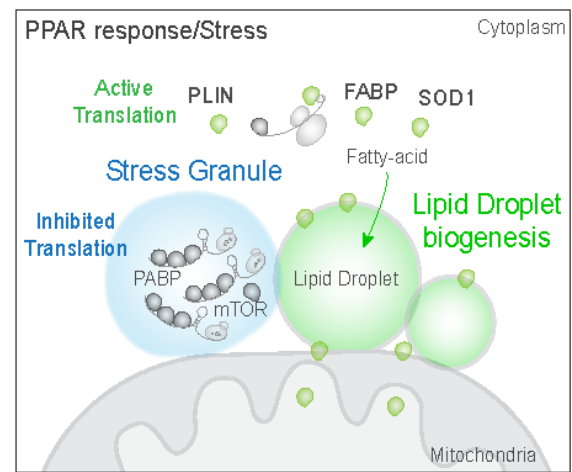




\section{Figure S1}

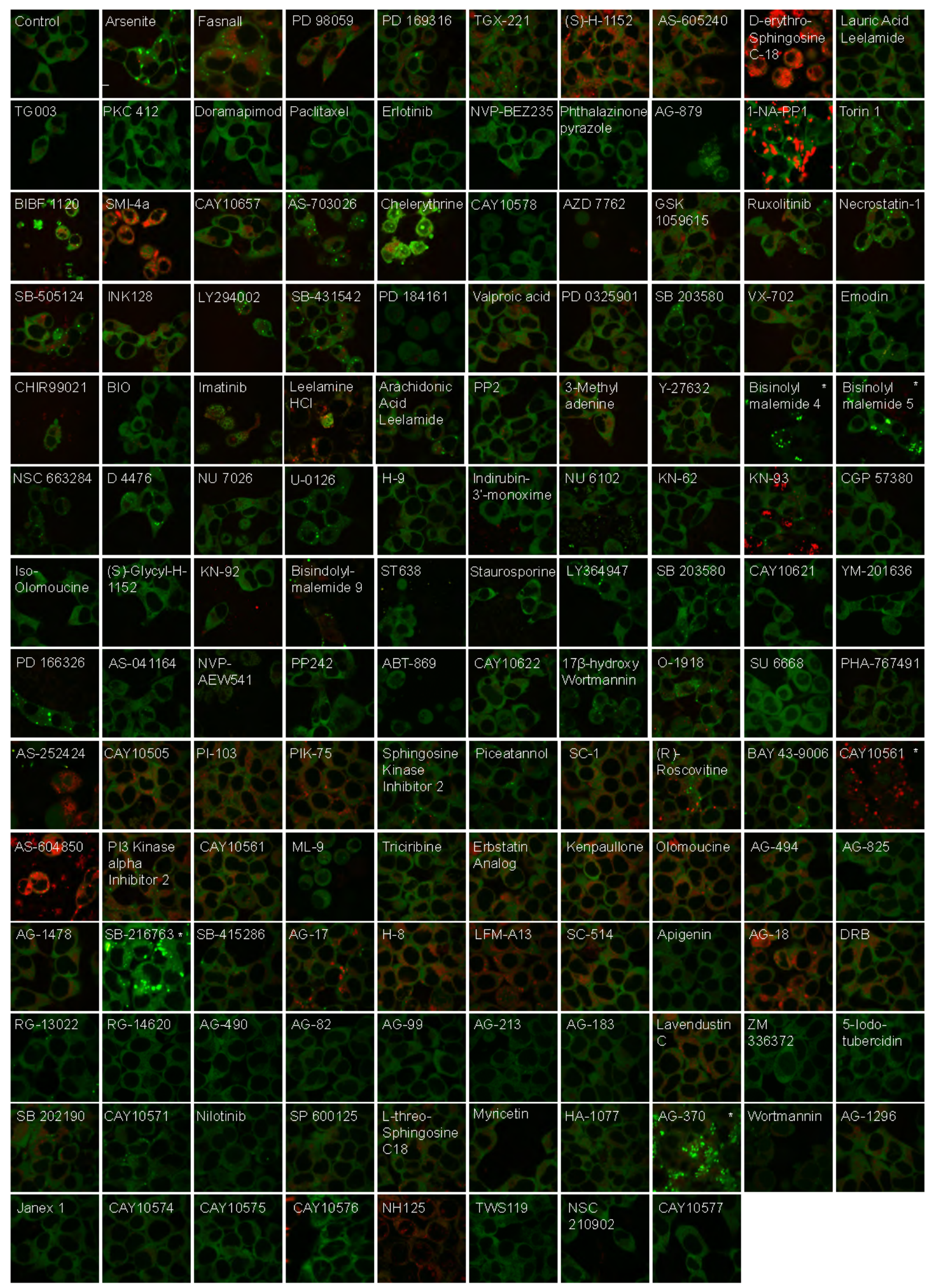


Figure S2

A

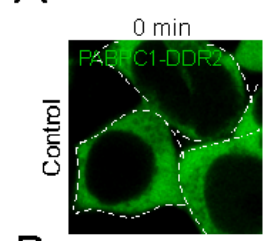

B

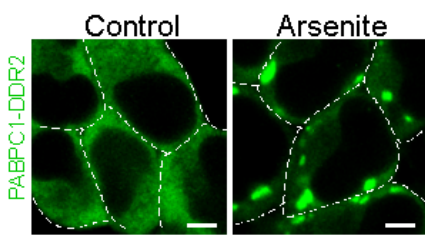

D

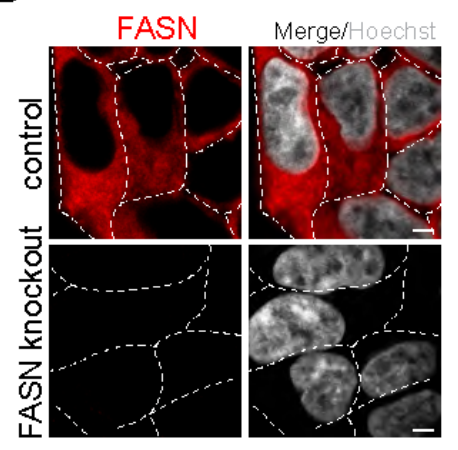

C

$90 \mathrm{~min}$
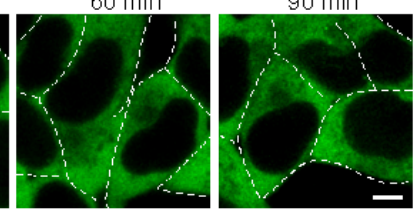

Fasnall

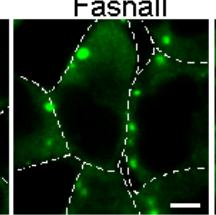

E

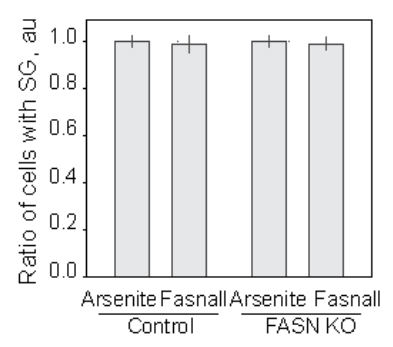

F
Fasnall
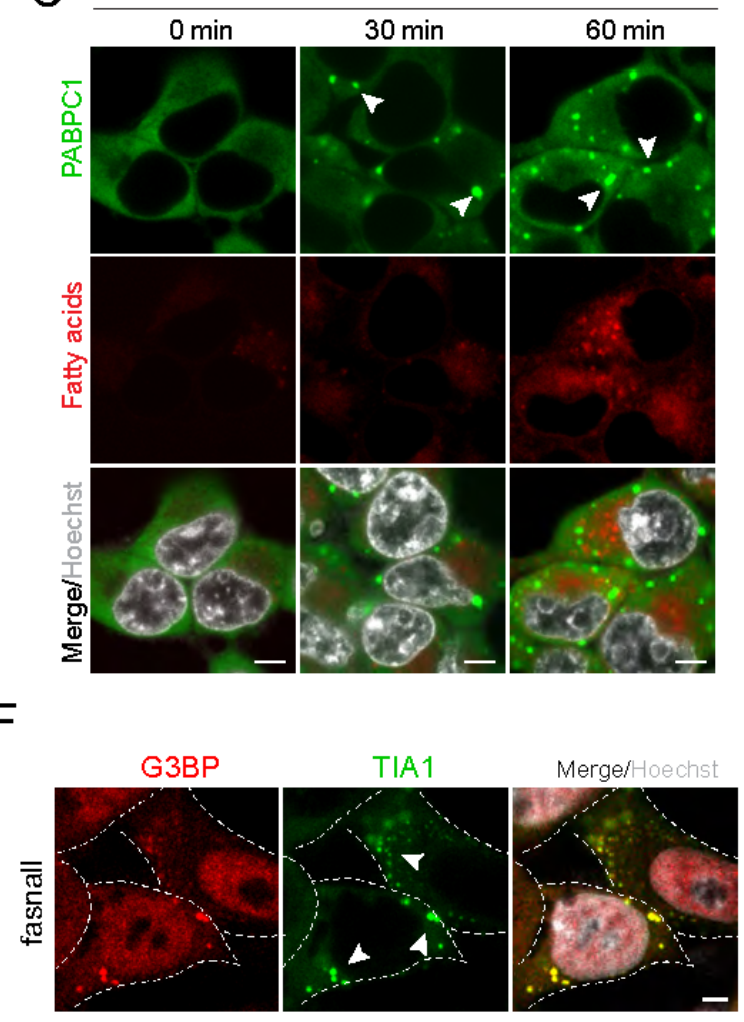


\section{Figure S3}
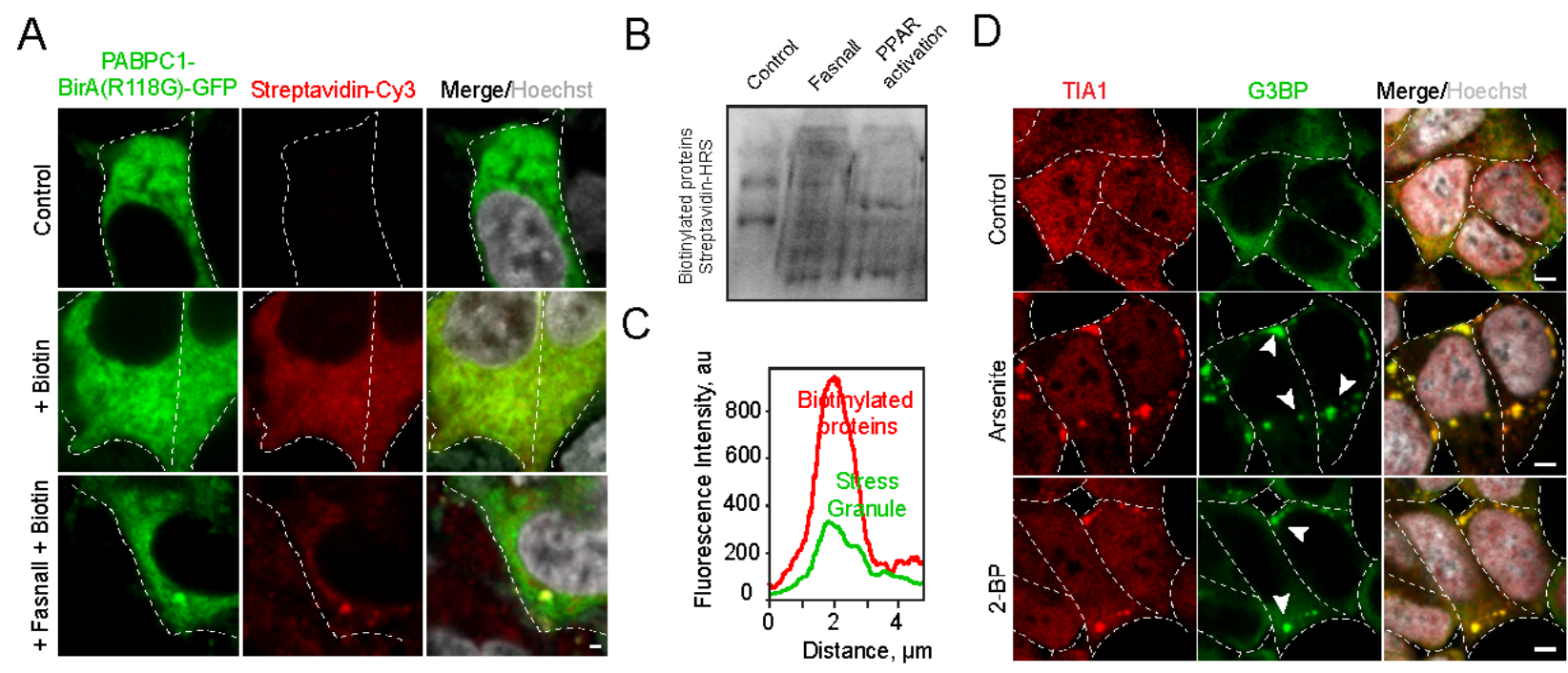


\section{Experimental Procedures}

\section{Cell culture and cell lines}

HEK293T cells were maintained in DMEM supplemented with 10\% fetal bovine serum (FBS), $1 \%$ penicillin/streptomycin, at $37^{\circ} \mathrm{C} / 5 \% \mathrm{CO} 2$, SH-SY5Y cells were maintained in 1:1 F12/DMEM media supplemented with $10 \% \mathrm{FBS}, 1 \%$ penicillin/streptomycin at $37^{\circ} \mathrm{C} / 5 \% \mathrm{CO} 2$. Cells modified via CRISPR/Cas9 were maintained as above with addition of puromycin $(2 \mu \mathrm{g} / \mathrm{ml}$, Sigma) during selection of clonal populations.

\section{Fatty acid import and quantification in Lipid Droplets}

Lipid droplets are pre-stained with either Bodipy (Green, $1 \mu \mathrm{M}$ Thermo Fischer Scientific) or Bodipy-C12 (Red, $1 \mu \mathrm{M}$ Thermo Fischer Scientific) for 30min. Fatty acids (bodipy-C12, $1 \mu \mathrm{M}$ ) were added 30 minutes prior to quantification (Holtta-Vuori et al., 2008). Accumulation of fatty acids in lipid droplets was measured as an increase of fluorescent intensity of a bodipy-FA dye. Fluorescence intensity was quantified inside lipid droplet area in 30 cells.

\section{Antibodies}

We used the following reagents to detect proteins: monoclonal anti-G3BP (Sigma-Aldrich WH0010146M1), polyclonal anti-TIA1 produced in rabbit (Sigma-Aldrich SAB4301803), antiGAPDH (sc-47724, Santa Cruz Biotechnology), anti PPARA (sc 398394, Santa Cruz Biotechnology), anti-PPARG (sc-7273X, Santa Cruz Biotechnology), anti-PPARD (sc74517, Santa Cruz Biotechnology), anti-mCherry (34974 Invitrogen), anti phospho-4EBP (Ser64, sc293124), anti-4EBP (sc 9977, Santa Cruz Biotechnology), anti-FASN (Sigma, SAB1403807).

Secondary antibodies for immunofluorescence: anti-Rabbit IgG Cy3-conjugated (Sigma-Aldrich C2306), anti-Mouse IgG Cy3-conjugated (Sigma-Aldrich C2181), anti-rabbit IgG Cy5 conjugated (Invitrogen A10523).

\section{Chemicals}

BODIPYтм 558/568 C12 (4,4-Difluoro-5-(2-Thienyl)-4-Bora-3a,4a-Diaza-s-Indacene-3Dodecanoic Acid, Thermo Fischer Scientific), Hoechst (Sigma), sodium arsenite (Fischer Chemical), cycloheximide (Sigma), BODIPY тм 493/503 (ThermoFischer Scientific, D3922), 
Streptavidin-Cy3 (Thermo Fischer Scientific), fatty acid free BSA (PAN), Rosiglitazone (Sigma), Clofibrate (Sigma), GW501516 (Sigma), UK5099 (Sigma), Etomoxir (Sigma), BPTES (Sigma), 2-bromopalmitic acid (Sigma), Streptavidin-HRP (Thermo Scientific), fatty acid free BSA (PAN Biotech), DMEM (PAN Biotech), FBS (PAN Biotech), PBS (PAN Biotech), methanol (Roth), Chlorophorm (Sigma), biotin (Sigma), aprotinin (Roth), leupeptin (Roth), Phenylmethylsulfonyl fluoride (PMSF, Sigma), fasnall (Sigma), C75 (Sigma), Kinase screening library (10505, Cayman Chemical)

\section{CRISPR/Cas9}

Knockout and endogenously tagged cell lines were constructed using CRISPR/Cas9 protocol and plasmids described in Ran et. al (Ran et al., 2013). Knockout cell lines were verified by western blotting, immunofluorescence. Genomic DNA was sequenced to verify disrupted region in knockout or fidelity of endogenous tagging. CRISPR specificity was profiled using DigenomeSeq web tool (http://www.rgenome.net/cas-offinder/) (Bae et al., 2014). Off targets were not found. The following target sequences are used to modify genomic DNA: knockout of PPARA CACAACCAGCACCATCTGGTCGCGA, knockout of FASN GAAGCTGCCAGAGTCGGAGAACTTGC.

\section{Plasmid Construction:}

All plasmids were constructed using Escherichia coli strain DH5a. Plasmids used in this study are summarized in the Table 1. We used px459 plasmid to clone CRISPR/Cas9 constructs for gene knockout, and px330 plasmid to construct plasmids used for endogenous tagging. pSpCas9(BB)2A-Puro (PX459) V2.0 was a gift from Feng Zhang (Addgene plasmid \# 62988; http://n2t.net/addgene:62988; RRID:Addgene_62988) (Ran et al., 2013). pX330-U6Chimeric_BB-CBh-hSpCas9 was a gift from Feng Zhang (Addgene plasmid \# 42230; http://n2t.net/addgene:42230; RRID:Addgene_42230) (Cong et al., 2013). Improved MS2 was cloned using pET251-pUC 12xMS2V6 Loxp KANr Loxp, which was a gift from Robert Singer (Addgene plasmid \# 104392; http://n2t.net/addgene:104392; RRID:Addgene_104392) (Tutucci et al., 2018). We fused 2 repeats of PPRE consensus sequence (aagtcaaaggtca), replacing CMV enhancer in pcDNA3.1, to construct the PPAR reporter plasmid (pPPAR-mCH). pCI-MS2V5PABPC1 was a gift from Niels Gehring (Addgene plasmid \# 65807; http://n2t.net/addgene:65807; RRID:Addgene_65807) (Fatscher et al., 2014). pcDNA3.1_FASN was a gift from Brijesh Kumar 
Singh (Addgene plasmid \# 107138; http://n2t.net/addgene:107138; RRID:Addgene_107138). Plasmid maps and cloning information is available upon request.

\begin{tabular}{|l|l|}
\hline Plasmid name & Source \\
\hline Px459-PPAR-KO-gRNA & This study \\
\hline Px330-FASN-KO-gRNA & This study \\
\hline pPPRE-mCH & This study \\
\hline pPPRE-SOD1-MS2-3'UTR & $\begin{array}{l}\text { This study, MS2 from } \\
\text { addgene plasmid \#104392 }\end{array}$ \\
\hline pPPRE-FASN-MS2-3'UTR & $\begin{array}{l}\text { This study, MS2 from } \\
\text { addgene plasmid \#107138 }\end{array}$ \\
\hline pcDNA3.1-MCP-GFP*2 & This study \\
\hline $\begin{array}{l}\text { pcDNA-PABPC1- } \\
\text { BirA(R118G)-GFP }\end{array}$ & $\begin{array}{l}\text { This study, PABPC1 from } \\
\text { addgene plasmid \#65807 }\end{array}$ \\
\hline
\end{tabular}

\section{MCP-MS2 experiment}

We used an improved MS2 binding sites fragment (MBSV6) which we fused to the SOD1 or FASN open reading frames (ORFs) 3' region (Tutucci et al., 2018). MS2-coat protein (MCP) fused to 2 GFP was co-transfected with MS2 constructs (Bernardi and Spahr, 1972).

\section{Extraction of biotinylated proteins}

Cells expressing PABPC1-BirA(R118G)-GFP were grown to 80-90\% confluency. SGs were induced with $100 \mu \mathrm{M}$ arsenite, $200 \mu \mathrm{M}$ 2-BP, or PPAR activation with $100 \mu \mathrm{M}$ rosiglitazone, clofibrate, and GW601516. After that, cells were incubated with biotin $(100 \mu \mathrm{M})$ for 4 hours, SGs formation was visualized on the microscope. Lysis and affinity capture were done according to Roux et al. with minor modifications (Roux et al., 2012). Cells were washed with PBS and (subsequent steps at $4^{\circ} \mathrm{C}$ ) lysed in the buffer $(50 \mathrm{mM}$ Tris $\mathrm{pH} 7.4,500 \mathrm{mM} \mathrm{NaCl}, 0.4 \% \mathrm{SDS}, 5 \mathrm{mM}$ EDTA, 1mM DTT, and protease inhibitor cocktail (aprotinin, leupeptin, and PMSF, 10 $\mu$ g) with glass beads $425-600 \mu \mathrm{m}$ (Sigma). After $1 \mathrm{~min}$ of vortex Triton X-100 was added to $2 \%$ concentration, after second round of vortex equal amount of 50mM Tris pH7.4 was added. After 
third round of vortex and centrifugation $5 \min 13000 \mathrm{rpm} 300 \mu \mathrm{l}$ of streptavidin-coated magnetic beads (NEB) were added to collected supernatants and incubated overnight at $4^{\circ} \mathrm{C}$ with agitation, $10 \%$ of the sample was collected for the Western Blot analysis. Beads were collected and (subsequent steps at room temperature) washed according to Roux et al.

\section{Preparing samples for the Mass spectroscopy}

The beads were washed free of detergents by two washes with $25 \mathrm{mM}$ Tris- $\mathrm{HCl} \mathrm{pH}$ 8.0. Then the packed beads were resuspended in $100 \mathrm{ul}$ of $8 \mathrm{M}$ urea, $10 \mathrm{mM}$ DTT, $25 \mathrm{mM}$ Tris-HCl pH 8.0 and incubated for $20 \mathrm{~min}$, followed by addition of iodoacetamide to a concentration of $55 \mathrm{mM}$ and incubation for $20 \mathrm{~min}$ in the dark. The urea was diluted by the addition of 6 volumes of $25 \mathrm{mM}$ Tris- $\mathrm{HCl} \mathrm{pH}$ 8.0, $0.25 \mu \mathrm{g}$ trypsin was added (Sigma) and the beads were incubated overnight at $37^{\circ} \mathrm{C}$ with gentle agitation. The released peptides were desalted by loading the whole bead supernatant on C18 Stage tips (Rappsilber et al., 2007). Eluted peptide material was used for MS analysis.

\section{LC/MS/MS analysis}

Protein digests were analyzed on a nanoflow chromatography system (Eksigent nanoLC425) hyphenated to a hybrid triple quadrupole-TOF mass spectrometer (TripleTOF 5600+) equipped with a Nanospray III ion source (Ionspray Voltage $2400 \mathrm{~V}$, Interface Heater Temperature $150^{\circ} \mathrm{C}$, Sheath Gas Setting 12) and controlled by Analyst TF 1.7.1 software build 1163 (all AB Sciex). In brief, peptides were dissolved in loading buffer ( $2 \%$ acetonitrile, $0.1 \%$ formic acid in water), enriched on a precolumn $(0.18 \mathrm{~mm}$ ID x $20 \mathrm{~mm}$, Symmetry C18, $5 \mu \mathrm{m}$, Waters, Milford/MA, U.S.A) and separated on an analytical RP-C18 column (0.075 mm ID x $250 \mathrm{~mm}$, HSS T3, $1.8 \mu \mathrm{m}$, Waters) using a 90 min linear gradient of 5-35\% acetonitrile/0.1\% formic acid (v:v) at $300 \mathrm{nl} \mathrm{min}^{-}$ 1 .

Qualitative LC/MS/MS analysis was performed using a Top25 data-dependent acquisition method with an MS survey scan of $\mathrm{m} / \mathrm{z} 350-1250$ accumulated for $350 \mathrm{~ms}$ at a resolution of 30,000 full width at half maximum (FWHM). MS/MS scans of m/z 180-1600 were accumulated for $100 \mathrm{~ms}$ at a resolution of 17,500 FWHM and a precursor isolation width of $0.7 \mathrm{FWHM}$, resulting in a total cycle time of 2.9 s. Precursors above a threshold MS intensity of $125 \mathrm{cps}$ with charge states 2+, $3+$, and $4+$ were selected for MS/MS, the dynamic exclusion time was set to $30 \mathrm{~s}$. MS/MS 
activation was achieved by CID using nitrogen as a collision gas and the manufacturer's default rolling collision energy settings. Two technical replicates per sample were acquired.

\section{MS data analysis}

Mass spectra data were processed using the MaxQuant computational platform, version 1.6.3.4 (Cox and Mann, 2008). Peak lists were searched against the human Uniprot FASTA sequence database (downloaded 02.01.19). The search included cysteine carbamidomethylation as a fixed modification and oxidation of methionine as variable modifications. Peptides with minimum of seven amino-acid length were considered and the required FDR was set to $1 \%$ at the peptide and protein level. Protein identifications required at least three unique or razor peptides per protein group. Relative protein quantification in MaxQuant was performed using the label free quantification (LFQ) algorithm (Cox et al., 2014). Identified proteins were analyzed with Perseus software (Tyanova et al., 2016). Gene ontology analysis was performed using STRING web tool (https://string-db.org/,(Szklarczyk et al., 2015).

\section{Microscopy}

For live cell imaging we used 4-well microscope glass bottom plates (IBIDI), or Cellview cell culture dish (Greiner Bio One). Confocal images and movies were acquired using a dual pointscanning Nikon A1R-si microscope equipped with a PInano Piezo stage (MCL), temperature and CO2 incubator, using a 60x PlanApo VC oil objective NA 1.40. We used 406nm, 488nm, 561nm, and 640nm laser (Coherent, OBIS). Movies for kymographs were acquired in resonant-scanning mode. Image processing was performed using NIS-Elements software (Nikon).

\section{Statistics and data analysis}

At least three independent biological and two technical repeats were performed to obtain the data. $P$ values were calculated by two-tailed Student t-test, or one-way ANOVA followed by a Turkey's post hoc test. The sample sizes were not predetermined. P-value $<0.05$ is considered statistically significant.

\section{Data availability}

Additional data that support the conclusions of this study are available on reasonable request. 


\section{References}

Alwarawrah, Y., Hughes, P., Loiselle, D., Carlson, D.A., Darr, D.B., Jordan, J.L., Xiong, J., Hunter, L.M., Dubois, L.G., Thompson, J.W., et al. (2016). Fasnall, a Selective FASN Inhibitor, Shows Potent Anti-tumor Activity in the MMTV-Neu Model of HER2(+) Breast Cancer. Cell Chem Biol 23, 678-688.

Ariumi, Y., Kuroki, M., Kushima, Y., Osugi, K., Hijikata, M., Maki, M., Ikeda, M., and Kato, N. (2011). Hepatitis $\mathrm{C}$ virus hijacks P-body and stress granule components around lipid droplets. J Virol 85, 68826892.

Bae, S., Park, J., and Kim, J.S. (2014). Cas-OFFinder: a fast and versatile algorithm that searches for potential off-target sites of Cas9 RNA-guided endonucleases. Bioinformatics 30, 1473-1475.

Bernardi, A., and Spahr, P.F. (1972). Nucleotide sequence at the binding site for coat protein on RNA of bacteriophage R17. Proc Natl Acad Sci U S A 69, 3033-3037.

Bischof, J., Salzmann, M., Streubel, M.K., Hasek, J., Geltinger, F., Duschl, J., Bresgen, N., Briza, P., Haskova, D., Lejskova, R., et al. (2017). Clearing the outer mitochondrial membrane from harmful proteins via lipid droplets. Cell Death Discov 3, 17016.

Boren, J., and Brindle, K.M. (2012). Apoptosis-induced mitochondrial dysfunction causes cytoplasmic lipid droplet formation. Cell Death Differ 19, 1561-1570.

Buchan, J.R., and Parker, R. (2009). Eukaryotic Stress Granules: The Ins and Outs of Translation. Molecular Cell 36, 932-941.

Buchan, J.R., Yoon, J.H., and Parker, R. (2011). Stress-specific composition, assembly and kinetics of stress granules in Saccharomyces cerevisiae. J Cell Sci 124, 228-239.

Cong, L., Ran, F.A., Cox, D., Lin, S., Barretto, R., Habib, N., Hsu, P.D., Wu, X., Jiang, W., Marraffini, L.A., et al. (2013). Multiplex genome engineering using CRISPR/Cas systems. Science 339, 819-823.

Contreras, A.V., Torres, N., and Tovar, A.R. (2013). PPAR-alpha as a key nutritional and environmental sensor for metabolic adaptation. Adv Nutr 4, 439-452.

Cox, J., Hein, M.Y., Luber, C.A., Paron, I., Nagaraj, N., and Mann, M. (2014). Accurate proteome-wide label-free quantification by delayed normalization and maximal peptide ratio extraction, termed MaxLFQ. Mol Cell Proteomics 13, 2513-2526.

Cox, J., and Mann, M. (2008). MaxQuant enables high peptide identification rates, individualized p.p.b.range mass accuracies and proteome-wide protein quantification. Nat Biotechnol 26, 1367-1372.

Dalen, K.T., Schoonjans, K., Ulven, S.M., Weedon-Fekjaer, M.S., Bentzen, T.G., Koutnikova, H., Auwerx, J., and Nebb, H.I. (2004). Adipose tissue expression of the lipid droplet-associating proteins S3-12 and perilipin is controlled by peroxisome proliferator-activated receptor-gamma. Diabetes 53, 1243-1252.

Fatscher, T., Boehm, V., Weiche, B., and Gehring, N.H. (2014). The interaction of cytoplasmic poly(A)binding protein with eukaryotic initiation factor $4 \mathrm{G}$ suppresses nonsense-mediated mRNA decay. RNA 20, 1579-1592.

Fournier, M.J., Coudert, L., Mellaoui, S., Adjibade, P., Gareau, C., Cote, M.F., Sonenberg, N., Gaudreault, R.C., and Mazroui, R. (2013). Inactivation of the mTORC1-eukaryotic translation initiation factor 4E pathway alters stress granule formation. Mol Cell Biol 33, 2285-2301. 
Gingras, A.C., Gygi, S.P., Raught, B., Polakiewicz, R.D., Abraham, R.T., Hoekstra, M.F., Aebersold, R., and Sonenberg, N. (1999). Regulation of 4E-BP1 phosphorylation: a novel two-step mechanism. Genes \& Development 13, 1422-1437.

Gorga, A., Rindone, G.M., Regueira, M., Pellizzari, E.H., Camberos, M.C., Cigorraga, S.B., Riera, M.F., Galardo, M.N., and Meroni, S.B. (2017). PPARgamma activation regulates lipid droplet formation and lactate production in rat Sertoli cells. Cell Tissue Res 369, 611-624.

Gubern, A., Barcelo-Torns, M., Casas, J., Barneda, D., Masgrau, R., Picatoste, F., Balsinde, J., Balboa, M.A., and Claro, E. (2009). Lipid droplet biogenesis induced by stress involves triacylglycerol synthesis that depends on group VIA phospholipase A2. J Biol Chem 284, 5697-5708.

Guil, S., Long, J.C., and Caceres, J.F. (2006). hnRNP A1 Relocalization to the Stress Granules Reflects a Role in the Stress Response. Molecular and Cellular Biology 26, 5744-5758.

Guo, Y., Cordes, K.R., Farese, R.V., Jr., and Walther, T.C. (2009). Lipid droplets at a glance. J Cell Sci $122,749-752$.

Heberle, A.M., Razquin Navas, P., Langelaar-Makkinje, M., Kasack, K., Sadik, A., Faessler, E., Hahn, U., Marx-Stoelting, P., Opitz, C.A., Sers, C., et al. (2019). The PI3K and MAPK/p38 pathways control stress granule assembly in a hierarchical manner. Life Sci Alliance 2.

Henne, W.M., Reese, M.L., and Goodman, J.M. (2018). The assembly of lipid droplets and their roles in challenged cells. EMBO J 37.

Holtta-Vuori, M., Uronen, R.L., Repakova, J., Salonen, E., Vattulainen, I., Panula, P., Li, Z., Bittman, R., and Ikonen, E. (2008). BODIPY-cholesterol: a new tool to visualize sterol trafficking in living cells and organisms. Traffic 9, 1839-1849.

Jevtov, I., Zacharogianni, M., van Oorschot, M.M., van Zadelhoff, G., Aguilera-Gomez, A., Vuillez, I., Braakman, I., Hafen, E., Stocker, H., and Rabouille, C. (2015). TORC2 mediates the heat stress response in Drosophila by promoting the formation of stress granules. J Cell Sci 128, 2497-2508.

Jin, Y., Tan, Y., Chen, L., Liu, Y., and Ren, Z. (2018). Reactive Oxygen Species Induces Lipid Droplet Accumulation in HepG2 Cells by Increasing Perilipin 2 Expression. Int J Mol Sci 19.

Kaganovich, D. (2017). There Is an Inclusion for That: Material Properties of Protein Granules Provide a Platform for Building Diverse Cellular Functions. Trends Biochem Sci 42, 765-776.

Kedersha, N., and Anderson, P. (2007). Mammalian stress granules and processing bodies. Methods Enzymol 431, 61-81.

Kedersha, N., Ivanov, P., and Anderson, P. (2013). Stress granules and cell signaling: more than just a passing phase? Trends Biochem Sci 38, 494-506.

Kedersha, N., Stoecklin, G., Ayodele, M., Yacono, P., Lykke-Andersen, J., Fritzler, M.J., Scheuner, D., Kaufman, R.J., Golan, D.E., and Anderson, P. (2005). Stress granules and processing bodies are dynamically linked sites of mRNP remodeling. J Cell Biol 169, 871-884.

Khong, A., Matheny, T., Jain, S., Mitchell, S.F., Wheeler, J.R., and Parker, R. (2017). The Stress Granule Transcriptome Reveals Principles of mRNA Accumulation in Stress Granules. Mol Cell 68, 808-820 e805.

Kim, Y.S., Lee, H.M., Kim, J.K., Yang, C.S., Kim, T.S., Jung, M., Jin, H.S., Kim, S., Jang, J., Oh, G.T., et al. (2017). PPAR-alpha Activation Mediates Innate Host Defense through Induction of TFEB and Lipid Catabolism. J Immunol 198, 3283-3295. 
Kobayashi, T., Winslow, S., Sunesson, L., Hellman, U., and Larsson, C. (2012). PKCalpha binds G3BP2 and regulates stress granule formation following cellular stress. PLoS One 7, e35820.

Krahmer, N., Farese, R.V., Jr., and Walther, T.C. (2013). Balancing the fat: lipid droplets and human disease. EMBO Mol Med 5, 973-983.

Li, Z., Thiel, K., Thul, P.J., Beller, M., Kuhnlein, R.P., and Welte, M.A. (2012). Lipid droplets control the maternal histone supply of Drosophila embryos. Curr Biol 22, 2104-2113.

Lindquist, S. (1981). Regulation of protein synthesis during heat shock. Nature 293, 311-314.

Lu, L., Han, A.P., and Chen, J.J. (2001). Translation Initiation Control by Heme-Regulated Eukaryotic Initiation Factor 2alpha Kinase in Erythroid Cells under Cytoplasmic Stresses. Molecular and Cellular Biology 21, 7971-7980.

Makishima, M., Tzeng, J., Byun, J., Park, J.Y., Yamamoto, T., Schesing, K., Tian, B., Sadoshima, J., and Oka, S.-i. (2015). An Ideal PPAR Response Element Bound to and Activated by PPARa. Plos One 10, e0134996.

Michelet, X., Dyck, L., Hogan, A., Loftus, R.M., Duquette, D., Wei, K., Beyaz, S., Tavakkoli, A., Foley, C., Donnelly, R., et al. (2018). Metabolic reprogramming of natural killer cells in obesity limits antitumor responses. Nat Immunol 19, 1330-1340.

Moldavski, O., Amen, T., Levin-Zaidman, S., Eisenstein, M., Rogachev, I., Brandis, A., Kaganovich, D., and Schuldiner, M. (2015). Lipid Droplets Are Essential for Efficient Clearance of Cytosolic Inclusion Bodies. Dev Cell 33, 603-610.

Nguyen, T.B., Louie, S.M., Daniele, J.R., Tran, Q., Dillin, A., Zoncu, R., Nomura, D.K., and Olzmann, J.A. (2017). DGAT1-Dependent Lipid Droplet Biogenesis Protects Mitochondrial Function during Starvation-Induced Autophagy. Dev Cell 42, 9-21 e25.

Nguyen, T.B., and Olzmann, J.A. (2017). Lipid droplets and lipotoxicity during autophagy. Autophagy 13, 2002-2003.

Olzmann, J.A., and Carvalho, P. (2019). Dynamics and functions of lipid droplets. Nat Rev Mol Cell Biol $20,137-155$.

Onal, G., Kutlu, O., Gozuacik, D., and Dokmeci Emre, S. (2017). Lipid Droplets in Health and Disease. Lipids Health Dis 16, 128.

Pennetta, G., and Welte, M.A. (2018). Emerging Links between Lipid Droplets and Motor Neuron Diseases. Dev Cell 45, 427-432.

Petan, T., Jarc, E., and Jusovic, M. (2018). Lipid Droplets in Cancer: Guardians of Fat in a Stressful World. Molecules 23.

Poulsen, L.1.C., Siersbæk, M., and Mandrup, S. (2012). PPARs: Fatty acid sensors controlling metabolism. Seminars in Cell \& Developmental Biology 23, 631-639.

Rakhshandehroo, M., Knoch, B., Muller, M., and Kersten, S. (2010a). Peroxisome proliferator-activated receptor alpha target genes. PPAR Res 2010.

Rakhshandehroo, M., Knoch, B., Müller, M., and Kersten, S. (2010b). Peroxisome Proliferator-Activated Receptor Alpha Target Genes. PPAR Research 2010, 1-20.

Rambold, A.S., Cohen, S., and Lippincott-Schwartz, J. (2015). Fatty acid trafficking in starved cells: regulation by lipid droplet lipolysis, autophagy, and mitochondrial fusion dynamics. Dev Cell 32, 678-692. 
Ran, F.A., Hsu, P.D., Wright, J., Agarwala, V., Scott, D.A., and Zhang, F. (2013). Genome engineering using the CRISPR-Cas9 system. Nat Protoc 8, 2281-2308.

Rappsilber, J., Mann, M., and Ishihama, Y. (2007). Protocol for micro-purification, enrichment, prefractionation and storage of peptides for proteomics using StageTips. Nat Protoc 2, 1896-1906.

Reineke, L.C., Cheema, S.A., Dubrulle, J., and Neilson, J.R. (2018). Chronic starvation induces noncanonical pro-death stress granules. J Cell Sci 131.

Rodriguez, M.A.D.R., and Kersten, S. (2017). Regulation of lipid droplet-associated proteins by peroxisome proliferator-activated receptors. Bba-Mol Cell Biol L 1862, 1212-1220.

Rohwedder, A., Zhang, Q., Rudge, S.A., and Wakelam, M.J. (2014). Lipid droplet formation in response to oleic acid in Huh-7 cells is mediated by the fatty acid receptor FFAR4. J Cell Sci 127, 3104-3115.

Rösch, K., Kwiatkowski, M., Hofmann, S., Schöbel, A., Grüttner, C., Wurlitzer, M., Schlüter, H., and Herker, E. (2016). Quantitative Lipid Droplet Proteome Analysis Identifies Annexin A3 as a Cofactor for HCV Particle Production. Cell Reports 16, 3219-3231.

Roux, K.J., Kim, D.I., Raida, M., and Burke, B. (2012). A promiscuous biotin ligase fusion protein identifies proximal and interacting proteins in mammalian cells. J Cell Biol 196, 801-810.

Sabatini, D.M. (2017). Twenty-five years of mTOR: Uncovering the link from nutrients to growth. Proc Natl Acad Sci U S A 114, 11818-11825.

San, Y.-Z., Liu, Y.U., Zhang, Y.U., Shi, P.-P., and Zhu, Y.-L. (2015). Peroxisome proliferator-activated receptor- $\gamma$ agonist inhibits the mammalian target of rapamycin signaling pathway and has a protective effect in a rat model of status epilepticus. Molecular Medicine Reports 12, 1877-1883.

Sengupta, S., Peterson, T.R., Laplante, M., Oh, S., and Sabatini, D.M. (2010). mTORC1 controls fastinginduced ketogenesis and its modulation by ageing. Nature 468, 1100-1104.

Sfakianos, A.P., Mellor, L.E., Pang, Y.F., Kritsiligkou, P., Needs, H., Abou-Hamdan, H., Desaubry, L., Poulin, G.B., Ashe, M.P., and Whitmarsh, A.J. (2018). The mTOR-S6 kinase pathway promotes stress granule assembly. Cell Death Differ 25, 1766-1780.

Sheikh, M.S., and Fornace, A.J., Jr. (1999). Regulation of translation initiation following stress. Oncogene $18,6121-6128$.

Shih, J.W., Wang, W.T., Tsai, T.Y., Kuo, C.Y., Li, H.K., and Wu Lee, Y.H. (2012). Critical roles of RNA helicase DDX3 and its interactions with eIF4E/PABP1 in stress granule assembly and stress response. Biochem J 441, 119-129.

Spriggs, K.A., Bushell, M., and Willis, A.E. (2010). Translational regulation of gene expression during conditions of cell stress. Mol Cell 40, 228-237.

Szklarczyk, D., Franceschini, A., Wyder, S., Forslund, K., Heller, D., Huerta-Cepas, J., Simonovic, M., Roth, A., Santos, A., Tsafou, K.P., et al. (2015). STRING v10: protein-protein interaction networks, integrated over the tree of life. Nucleic Acids Res 43, D447-452.

Takahara, T., and Maeda, T. (2012). Transient sequestration of TORC1 into stress granules during heat stress. Mol Cell 47, 242-252.

Teleman, A.A. (2005). 4E-BP functions as a metabolic brake used under stress conditions but not during normal growth. Genes \& Development 19, 1844-1848. 
Thedieck, K., Holzwarth, B., Prentzell, M.T., Boehlke, C., Klasener, K., Ruf, S., Sonntag, A.G., Maerz, L., Grellscheid, S.N., Kremmer, E., et al. (2013). Inhibition of mTORC1 by astrin and stress granules prevents apoptosis in cancer cells. Cell 154, 859-874.

Tsai, T.Y., Wang, W.T., Li, H.K., Chen, W.J., Tsai, Y.H., Chao, C.H., and Wu Lee, Y.H. (2017). RNA helicase DDX3 maintains lipid homeostasis through upregulation of the microsomal triglyceride transfer protein by interacting with HNF4 and SHP. Sci Rep 7, 41452.

Tutucci, E., Vera, M., Biswas, J., Garcia, J., Parker, R., and Singer, R.H. (2018). An improved MS2 system for accurate reporting of the mRNA life cycle. Nat Methods 15, 81-89.

Tyanova, S., Temu, T., Sinitcyn, P., Carlson, A., Hein, M.Y., Geiger, T., Mann, M., and Cox, J. (2016). The Perseus computational platform for comprehensive analysis of (prote)omics data. Nat Methods 13, 731-740.

VandeKopple, M.J., Wu, J., Auer, E.N., Giaccia, A.J., Denko, N.C., and Papandreou, I. (2019). HILPDA regulates lipid metabolism, lipid droplet abundance and response to microenvironmental stress in solid tumors. Mol Cancer Res.

Varga, T., Czimmerer, Z., and Nagy, L. (2011). PPARs are a unique set of fatty acid regulated transcription factors controlling both lipid metabolism and inflammation. Biochim Biophys Acta 1812, 1007-1022.

Velazquez, A.P., and Graef, M. (2016). Autophagy regulation depends on ER homeostasis controlled by lipid droplets. Autophagy 12, 1409-1410.

Walther, T.C., Chung, J., and Farese, R.V., Jr. (2017). Lipid Droplet Biogenesis. Annu Rev Cell Dev Biol 33, 491-510. 


\section{Chapter 4}

\section{Eisosome regulation of Stress Granule formation in yeast Saccharomyces cerevisiae}

SGs formation occurs in all eukaryotic cells. In this chapter we examine SG formation in yeast $S$. cerevisiae subjected to glucose starvation. Yeast cells are a particularly good tool to study cellular adaptation, since growth over many generations can be directly assessed. Therefore, we also examine SG functioning in stress adaptation. 


\section{Summary}

As the physical barrier between the cell and the outside environment, the plasma membrane is well-positioned to be the first responder to stress. The membrane is also highly vulnerable to many types of perturbation, including heat, force, osmotic pressure, lipid shortage, and starvation. To determine whether the structural changes in the plasma membrane of Saccharomyces cerevisiae brought about by nutrient stress can be communicated to regulatory networks within the cell, we identified proteins that interact with stress granules (SGs), subcellular structures composed of proteins and nontranslated RNAs that form when cells are stressed. We found that SG proteins interacted with components of eisosomes, which are subcortical membrane structures with a distinct lipid and protein composition. In response to starvation-triggered phosphorylation of eisosome proteins, eisosomes clustered and recruited SG components, including active Pkc1. The absence of eisosomes impaired SG formation, resulting in delayed recovery from nutrient deprivation. Thus, eisosome clustering represents an example of inter-domain communication in response to stress and identifies a previously unknown mechanism of SG regulation.

\section{Introduction}

Adaptation to stress requires communication between cellular compartments. Of all of these, the role of the plasma membrane in triggering stress responses is one of the least well understood, despite the fact that it is the location where many stresses are likely to be first detectable (1). Much like the cytoplasm, the plasma membrane is partitioned into discrete functional domains, some of which undergo structural changes in response to specific external and intracellular changes (2-5). In the yeast Saccharomyces cerevisiae one of the domains that has been most extensively studied in the context of stress response is the eisosome compartment ( 6 ).

Eisosomes are filament-like multiprotein complexes that localize radially throughout the membrane in fungi (6-9). The two main eisosome components, Pill (phosphorylation inhibited by long-chain bases 1) and Lsp1 (long-chain bases stimulate phosphorylation 1), grip the plasma membrane with groove-like Bin/amphiphysin/Rys $(\mathrm{BAR})$ domains $(10,11)$ and polymerize into cylindrical structures in vitro (10). Eisosome assembly and disassembly is regulated by the phosphorylation of Pill and Lsp1 by major regulatory kinases, including Pkh1 (protein kinase b- 
activating kinase homolog 1$)$ and $\operatorname{Pkh} 2(\operatorname{Pkh} 1 / 2)(12,13)$ and kinases that are activated by protein kinase C 1 (Pkc1) (14), as well as by other eisosome components (15). Although eisosomes are not essential and appear to lack a clearly defined cellular function, they have sparked interest due to their pronounced re-organization during stress and their association with nutrient transporters and protein components of ribonucleoprotein (RNP) granules during starvation, all of which suggests that they may play a protective role in stress responses (16-18).

Several functions have been proposed for eisosomes, including regulation of cell wall synthesis (19), storing a reservoir of plasma membrane in their furrows in order to withstand sheer stress $(6,20)$, and regulating RNA decay by recruiting the P body protein Xrn1 (7). The strongest available evidence indicates that eisosomes play a protective role during metabolic stress (16-18). The lateral microdomains that are formed by eisosome assembly protect nutrient transporters that are localized there during nutrient stress due to endocytosis and degradation $(17,18)$. Eisosome formation is sensitive to nutrient availability and the abundance of cellular sphingolipids, and can in turn modulate the activity of the nutrient stress mediator target of rapamycin complex 2 (TORC2) (21-24). This leads to the suggestion that eisosomes may constitute part of a signaling hub for relaying nutrient starvation stress to other compartments.

Here we investigated the role of eisosome signaling in the nutrient starvation stress response and show that eisosome clustering directly primed the assembly of sub-cortical stress granules (SGs). SGs respond to a variety of stress signals, propagating them across multiple levels of cellular regulation (25-29). Due to the presence of non-translated RNAs, ribosomal subunits, and translation initiation factors in SGs, these structures have been thought to repress translation and perhaps store mRNAs for future use (30-32). SGs can also recruit and modulate the activities of key signaling proteins, including the nutrient sensor TOR and PKC (32-35). We found that eisosomes underwent Pkc1-dependent clustering during starvation. Eisosome rearrangement promoted SG assembly, which in turn recruited more active Pkc1. The ablation of eisosomes severely hampered SG formation and resulted in a substantial delay of recovery from nutrient deprivation. 


\section{Results}

Stress Granules associate with eisosomes during starvation

We investigated the subcellular architecture of SGs in the context of glucose starvation (32). In order to uncover transient and peripheral SG interactors, we optimized the proximitydependent biotinylation (BioID) approach for use in yeast (Fig. 1A; fig. S1, A-C) (36). This approach allowed us to visualize SG formation while simultaneously inducing the biotinylation of SG components and nearby proteins in live cells (fig. S1, A-C). We used fusions of two independent SG components (Pub1 and Pab1) to the biotin ligase BirA for biotinylating nearby proteins (Fig. 1A; fig. S1E). By subtracting the control interactome from the starvation interactome, we identified a set of candidate SG components and interactors that were either coassembled with or located proximal to SGs (table S3; Fig. 1B). We found multiple interactions between SGs and the eisosome complex (Fig. 1, B-C), as well as the kinase Pkh2, which localizes to eisosomes and regulates eisosome assembly $(13,14,37,38)$. We also observed a strong enrichment of Pkc1 in SGs, which is consistent with the reported localization of PKC to SGs in mammalian cells (39), together with components of the actin cytoskeleton, actin bodies (Figure S1K) (40).

We confirmed the interaction between SGs and eisosomes with confocal and structured illumination microscopy (SIM) and bimolecular fluorescence complementation (BiFC) in live cells experiencing starvation stress (Fig. 1, D-F; fig.S1D, distance between eisosomes and SGs $250 \mathrm{~nm}$ ). Eisosomes were consistently associated with SGs, when visualizing the eisosome components Pil1 or Lsp1, during acute starvation or chronic stationary-phase starvation (fig. S1, F-G). The majority of SGs that formed during starvation did so in contact with eisosomes, suggesting that the interaction was not a random phenomenon (Figure 1D, F); $67 \%$ of the SGs that formed in cells were proximal to eisosomes, as calculated by measuring the overlap in fluorescence intensity peaks (fig. S1H). We also tested whether SGs associated with eisosomes when they first appeared during starvation (fig. S1, H and I). SGs remained in proximity to eisosomes with little to no movement from the beginning of starvation stress and for 2 hours thereafter (fig. S1, I and J). Eisosomes were also stable during the course of starvation (fig. S3F).

We then examined the nature of the SG-eisosome interaction. BiFC showed that the SG marker, polyA binding-protein 1 (Pab1), was bound to the eisosome core component Pill during 
SG assembly (Fig. 1E; fig. S1L). We asked whether abolishing eisosome complexes by deleting both Pill and Lsp1 had an effect on SG formation. Indeed, cells without eisosomes had a substantial defect in SG formation (Fig. 1, G-H; fig S1M). Complementation of cells in which

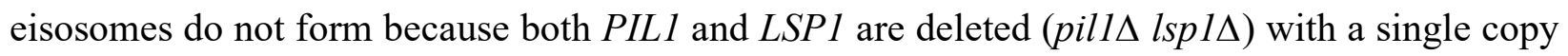
of PIL1-mCherry $(\mathrm{mCH})$ restored SG formation during starvation (Fig. 1, G and I). Cell lacking eisosomes showed a non-significant decrease in Pab1 amounts; however, a similar decrease in Pab1 during starvation did not impair SG formation in the wild-type strain, and so could not alone

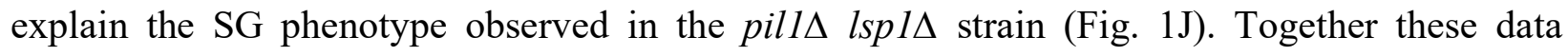
suggested to us that the eisosome complex plays a direct role in SG formation, and we set out to examine the mechanism of how it may do so.

\section{Eisosome clustering promotes Stress Granule formation}

We observed that the distance between eisosomes decreased under glucose starvation compared to non-starved conditions (Fig. 2, A-B), yet the amount of Pill in cells remained constant (Figure 2C). Measuring the average minimal distance between eisosomes on a radial cell section (fig. S2B), we noticed that, upon glucose depletion, Pill foci become closer to each other, occasionally forming elongated fibril-like structures (Fig.2,A and B; fig. S2, A and B). Total internal reflection - structured illumination (TIRF-SIM) imaging in live cells confirmed the pronounced clustering between eisosomes (Fig. 2A; fig. S2A). Eisosome assembly is known to be regulated by phosphorylation $(13,37,38)$. Mass spectroscopy phosphopeptide analysis showed that Pill was phosphorylated at $\operatorname{Ser}^{163}$ and $\operatorname{Ser}^{230}$ during glucose starvation (Fig. 2D; Table S3). We constructed phospho-null S163A and S230A and phospho-mimetic S163D and S230D mutant strains by complementing the pill $1 \Delta$ sp $1 \Delta$ strain with a single copy of each engineered form of PIL1 (fig.S2C; fig.S3D). Phospho-null Pill overexpression in the eisosome-free background led to impaired eisosome assembly and reduced the formation of SGs during starvation (Fig. 2, E-F). The phospho-mimetic mutant S230D restored the shorter spacing between eisosomes during starvation as well as the wild-type SG phenotype (Fig. 2, E-F). Additionally, we tested the Cterminal disordered region of Pill, because disordered regions play a role in protein aggregation and clustering, particularly in the case of SGs $(41,42)$. Complementation of the eisosome-deficient

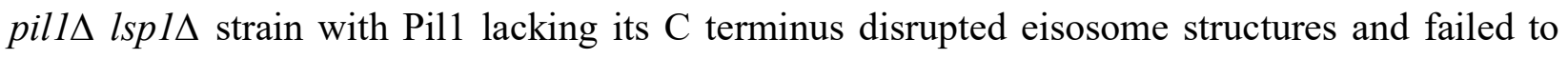


nucleate SG assembly during starvation (Fig. 2, E-F). Overall, we found a correlation between SG formation and the average minimal distance between eisosomes (clustering reduces the distance) to be -0.74 (Pearson correlation coefficient). Additionally, overexpression of high amounts of wild-type Pill resulted in the formation of cytoplasmic fibrils that primed Pab1 assembly (fig. $\mathrm{S} 2 \mathrm{D})$. Reconstituting eisosomes by complementation in pills $l s p 1 \Delta$ cells also restored SG formation during starvation (Fig. 2E). Thus, eisosome clustering is a bona fide adaptive response to stress, possibly promoting cellular fitness by priming SG assembly. We also observed that the ability to trigger eisosome clustering during starvation was linked to the replicative age of cells in a dividing yeast population (fig. S2E).

\section{Eisosomes undergo Pkc1-dependent rearrangements during starvation}

We next looked at the molecular mechanism of Pill-dependent eisosome rearrangement. Pill is phosphorylated in a Pkc1-dependent manner (13), and Pkcl itself is activated by the kinases Pkh1/2, which are associated with eisosomes (14). We were therefore confronted with the intriguing possibility that Pkcl-dependent phosphorylation triggers Pill clustering. Pkc1 is an essential signaling protein involved in polarized growth and division, activating downstream kinases of the mitogen-activated protein kinase (MAPK) module, including the MAPK Slt2. In order to test this feed-forward loop model of Pkc1-mediated Pill phosphorylation promoting eisosome reorganization and SG formation, we constructed a yeast strain in which Pkc1 was tagged with an auxin-inducible degron (AID) and an integrated copy of the OsTIR1 (43), allowing us to conditionally deplete cells of Pkc1 by treating them with auxin (fig. S2, F-G; Fig. 2, G-H). As suggested by the model, Pkc1 depletion abolished eisosome clustering (Fig. 2, G-H). We next asked whether phospho-mimetic mutants of Pill could bypass the Pkc1-dependent inhibition of clustering. Indeed, overexpression of S163D or S230D mutant forms of Pill restored wild-type spacing between eisosomes in response to starvation (fig. S2, I and J). Although it is possible that Pil1 is phosphorylated by downstream kinases of the MAPK module that is activated by Pkc1, and not by Pkc1 itself, we ruled out Slt2 as mediating this modification (fig. S2H). Thus, we argue that Pkc1-dependent phosphorylation of Pil1 - and perhaps direct phosphorylation of Pill by Pkc1 results in eisosome clustering during starvation. 


\section{Active Pkc1 is recruited to Stress Granules}

PKC, the mammalian homolog of Pkc1, has been shown to associate with SGs in mammalian cells (33). Our BioID analysis showed that Pkc1 is also a SG component in yeast (Fig. 3, A-B; fig. S3A). Thus, we hypothesized that Pkc1-dependent Pill phosphorylation primes SG nucleation by recruiting additional Pkc1. A key prediction of our feed-forward loop model, therefore, is that the Pkc1 recruited to SGs is in an active state. To test whether the active form of Pkc1 was preferentially recruited to SGs, we constructed a strain expressing a copy of Pkc1 R398P, a constitutively active form (Fig. 3A) (44). Overexpression of Pkc1 R398P promoted SG formation, and Pkc1 R398P was also preferentially incorporated into SGs during starvation compared to wild-type Pkc1 (Fig. 3B; fig. S3C), though it was also somewhat toxic. Wild-type Pkc1 exhibited pronounced toxicity in the eisosome-deficient pills lsp $1 \Delta$ strain (Fig. 3C; fig. S3B).

Overexpression of active Pkc1 restored SG formation during starvation to a wild-type amount in cells lacking eisosomes (pills $l_{s p} 1 \Delta$ ), indicating that $\mathrm{Pkc} 1$ activation was sufficient to drive SG formation (Fig. 3D). The links between eisosomes and SGs, therefore, may be Pkh1/2, which can activate Pkc1 and also localize to eisosomes (14, 37). Indeed, a Pkc1 T983A mutant that is not phosphorylated by Pkh2 (14) remained trapped on the membrane and in nuclei (Fig. 3, E; fig. S3G-I) and did not localize to SGs.

\section{Pkc1 stored in Stress Granules rescues cells after starvation}

To further confirm that Pkc1 is recruited to SGs in its active form , we constructed a livecell Pkc1 activity fluorescence resonance energy transfer (FRET) reporter by fusing Ruby2 and Clover to the $\mathrm{N}$ and $\mathrm{C}$ termini of Pkc1, respectively (Fig. 3F) (45). The whole-cell average amounts of Pkc1 activity, as measured by Clover/Ruby2 fluorescence intensity ratio with a single $488 \mathrm{~nm}$ excitation, decreased after 1 hour of starvation, indicating an overall decrease in Pkc1 activity (Fig. 3, G and H). The Pkc1 pool localized to SGs, on the other hand, was in an active state, comparable to that of the positive control (Pkc1 activated by caspofungin (46)) (Fig. 3I; fig. S3E). This further reinforces the idea that SGs sequester active Pkc1, either to reduce its activity elsewhere in the cytoplasm, to store active $\mathrm{Pkc1}$ for a burst of activity following stress resolution, or to create a 
high local concentration of active enzyme, thereby promoting further eisosome clustering and amplifying the stress signal (Fig. 3J). Pkcl is necessary for growth and localizes to the bud neck during cell division to promote mitosis (47). In order to understand the functional role of active Pkc1 storage in SGs we followed the pool of fluorescently tagged Pkc1 (Pkc1- $\mathrm{mCH})$ during longterm starvation and recovery (Fig. 4A). We found that, in recovery after starvation, Pkc1 was able to return to the bud neck after SG clearance in wild-type cells (Fig. 4, A and B). In cells lacking eisosomes, however, Pkc1 failed to relocalize to the bud neck after starvation, likely due to a clear decrease in the amount of tagged protein (Fig. 4B). Hence, without eisosomes and SGs, cells would have to re- synthesize Pkc1 after long-term starvation in order to accumulate the Pkc1 necessary to re-start division. Not surprisingly, therefore, when we monitored cell growth for 24 hours of recovery from 24 hours in stationary phase, we observed a dramatic delay in resumption of mitosis in eisosome-deleted cells (Fig. 4, C and D).

\section{Discussion}

Efficient adaptation to stress requires integration of several stress response pathways. Here we showed that in response to nutrient starvation stress, the structural rearrangement of eisosomes promoted $\mathrm{SG}$ formation. One of the key fitness benefits of SG formation in this context appears to be in enabling a faster re-initiation of cell division following stress resolution. We suggest that the eisosome-SG-Pkc1 interaction functions as a feed-forward loop, whereby $\mathrm{Pkc1}$ is responsible for the initial clustering of Pill, which then primes SG formation by recruiting Pkc1, which in turn promotes SG formation (Fig. 3J). Given that Pkc1 is generally in low abundance in the cell and that active Pkc1 is rapidly turned over $(48,49)$, a feed-forward loop is able to rapidly amplify a local stress signal, resulting in a broad reorganization of cellular resources and saving Pkc1 from degradation (Fig. 3J). This in turn allowed cells to restart their growth immediately after SG clearance. In the absence of eisosomes, SGs were less abundant, and without them Pkc1 was not protected from degradation, which resulted in a delayed recovery after long-term starvation (Fig. 4D).

Our findings resonate with much that is already known about eisosomes, including that Pil1 is phosphorylated in a Pkc1-dependent manner $(13,37)$, and that phosphorylation induces 
eisosome rearrangements (37). An increase in eisosome density during metabolic stress has also been reported (17). The data that we show here add mechanistic detail and extend the functional implications of eisosome dynamics. Our study also presents a previously unknown functionality for SGs and perhaps other RNP granules. Pkc1 promotes RNP granule formation in yeast and mammalian cells $(12,39)$, and we demonstrated that overexpression of active Pkc1 complemented the SG formation defect in an eisosome-deficient strain. We argue that localization of Pkh2 to eisosomes promotes local Pkcl activation, which in turn induces SG formation, which selfamplifies by recruiting more active Pkcl.

Active Pkc1 is normally degraded by the ubiquitin-proteasome system with rapid kinetics (49). This is particularly important in light of the known role of eisosomes in protecting nutrient transporters, including the arginine permease Can1, from degradation $(17,18)$. An increase in eisosome density during starvation leads to trans-membrane clustering of Can1, which protects this permease from endocytosis and lysosomal degradation (17). Our study showed that eisosomes also regulated the sub-cortical recruitment of active Pkcl to SGs, where it was protected from turnover and stored until the possibility of growth is restored. Additionally, in Candida albicans, eisosome complexes help alleviate copper toxicity by maintaining the lipid content of the plasma membrane (50). Taken together, this evidence opens up the possibility of a general protective role for eisosomes during metabolic stress $(16,18)$.

In addition to illuminating the cellular role of eisosomes in fungi, our model has implications for understanding the biology of SGs and other RNP granules in yeast as well as in other organisms. By providing a specific context for SG formation within the cellular architecture of yeast, our data raise the question of whether other SG nucleation sites exist in different cellular compartments and whether such nucleation sites might be a universal strategy for differentiating between compartment-specific and cell-wide stress responses.

\section{Materials and Methods}

\section{Strains and manipulations}

We used standard conditions for culturing yeast and bacterial cells (51). Yeast strains used in this study are based on BY4741 or W303 strains $(52,53)$. We introduced deletions using PCR based 
deletion strategy (54). Gene deletions were verified by PCR using a forward primer upstream of the reading frame and a reverse primer inside the marker reading frame. Yeast transformations were performed using LiAc/PEG method (55) with minor modifications (56). The strains used in the study are summarized in table S1. We used copper-inducible expression of PILI. We verified that the levels of copper were not causing additional toxicity to the eisosome-lacking strains as was demonstrated for C. albicans (50) (Fig. S3D).

\section{Plasmid construction}

All plasmids were constructed using Escherichia coli strain DH5a. Integration was performed using pDK plasmids as backbones $(49,56)$. Plasmids used in this study are summarized in table S2. Site-directed mutagenesis was verified by sequencing. Plasmids for endogenous tagging were constructed by modifying pKT127 plasmid (57).

\section{Protocol for stress granule induction}

Freshly grown yeast culture was diluted and grown in synthetic defined (SD) media to mid log phase. Cells are pelleted and the media is changed to SD without glucose for the time indicated in the experiment, usually 1 hour. To induce a more prominent SG response, the cells can be transferred to $37^{\circ} \mathrm{C}$ during starvation (used for SIM and TIRF/SIM imaging). Cells with SG are visualized in the same media, changing the media to glucose will result in fast clearance of inclusions.

\section{Extraction of biotinylated proteins}

Yeast strain expressing PAB1-BirA(R118G)-GFP or PUB1- BirA(R118G)-GFP was grown overnight in YPD media followed by 8 hours in biotin depleted SD media (Formedium). After that, biotin $(100 \mu \mathrm{M})$ was added to the media and cells were grown either in glucose depleted media with subsequent heat stress or SD complete media for $6 \mathrm{~h}$. SG formation was visualized on the microscope. Cells were harvested and frozen. Lysis and affinity capture were done according to Roux et al. with minor modifications (36). Cells were washed with water and (subsequent steps at $\left.4^{\circ} \mathrm{C}\right)$ lysed in the buffer $(50 \mathrm{mM}$ Tris $\mathrm{pH} 7.4,500 \mathrm{mM} \mathrm{NaCl}, 0.4 \% \mathrm{SDS}, 5 \mathrm{mM}$ EDTA, $1 \mathrm{mM}$ DTT,

and 1x ProBlock Gold yeast protease inhibitor cocktail (GoldBio)) with glass beads 425-600 $\mu \mathrm{m}$ (Sigma). After $1 \mathrm{~min}$ of vortex Triton X-100 was added to $2 \%$ concentration, after second round of vortex equal amount of $50 \mathrm{mM}$ Tris $\mathrm{pH} 7.4$ was added. After third round of vortex and 
centrifugation, streptavidin-coated magnetic beads (NEB) were added to the supernatants and incubated overnight at $4{ }^{\circ} \mathrm{C}$ with agitation. Beads were collected and washed according to Roux et al.

\section{Preparing samples for mass spectroscopy}

The beads were washed free of detergents by two washes with $25 \mathrm{mM}$ Tris- $\mathrm{HCl} \mathrm{pH} \mathrm{8.0.} \mathrm{Then} \mathrm{the}$ packed beads were resuspended in $100 \mathrm{ul}$ of $8 \mathrm{M}$ urea, $10 \mathrm{mM}$ DTT, $25 \mathrm{mM}$ Tris- $\mathrm{HCl} \mathrm{pH} 8.0$ and incubated for $20 \mathrm{~min}$, followed by addition of iodoacetamide to a concentration of $55 \mathrm{mM}$ and incubation for $20 \mathrm{~min}$ in the dark. The urea was diluted by the addition of 6 volumes of $25 \mathrm{mM}$ Tris- $\mathrm{HCl} \mathrm{pH}$ 8.0, $0.25 \mu \mathrm{g}$ trypsin was added (Promega Corp., Madison, WI, USA) and the beads were incubated overnight at $37^{\circ} \mathrm{C}$ with gentle agitation. The released peptides were desalted by loading the whole bead supernatant on C18 Stage tips (58). Two thirds of the eluted peptide material were used for MS analysis.

For phosphoprotein analysis, the same protocol of reduction, alkylation, and trypsin digestion was followed, but the phospho-peptides were subjected to enrichment by $\mathrm{TiO}_{2}$ resin (Calbochem ProteoExtract kit) and then desalted on stage tips.

\section{LC MS/MS analysis}

MS analysis was performed using a Q Exactive Plus mass spectrometer (Thermo Fisher Scientific) coupled on-line to a nanoflow UHPLC instrument (Ultimate 3000 Dionex, Thermo Fisher Scientific). Eluted peptides were separated over a 150-min gradient run at a flow rate of $0.3 \mathrm{ul} / \mathrm{min}$

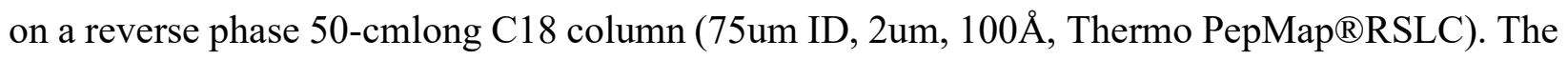
survey scans (380-2,000 m/z, target value 3E6 charges, maximum ion injection times $50 \mathrm{~ms}$ ) were acquired and followed by higher energy collisional dissociation (HCD) based fragmentation (normalized collision energy 28). A resolution of 70,000 was used for survey scans and up to 15 dynamically chosen most abundant precursor ions were fragmented (isolation window $1.6 \mathrm{~m} / \mathrm{z}$ ). The MS/MS scans were acquired at a resolution of 17,500 (target value 1E5 charges, maximum ion injection times $120 \mathrm{~ms})$.

For samples of $\mathrm{TiO}_{2}$-enriched phosphopeptides, the MS settings were modified as follows: for survey scans the maximum ion injection time was $200 \mathrm{~ms}$, MS/MS scans were acquired at a resolution of 35,000 (target value 2E5 charges, maximum ion injection times $200 \mathrm{~ms}$ ). 


\section{MS data analysis}

Mass spectra data were processed using the MaxQuant computational platform, version 1.5.3.12 (59). Peak lists were searched against the Saccharomyces cerevisiae Uniprot FASTA sequence database containing a total of 9,591 reviewed entries. The search included cysteine carbamidomethylation as a fixed modification and oxidation of methionine as variable modifications. Peptides with minimum of seven amino-acid length were considered and the required FDR was set to $1 \%$ at the peptide and protein level. Protein identification required at least 3 unique or razor peptides per protein group. Relative protein quantification in MaxQuant was performed using the label free quantification (LFQ) algorithm (60). LFQ in MaxQuant uses only common peptides for pair-wise ratio determination for each protein and calculates a median ratio to protect against outliers. It then determines all pair-wise protein ratios and requires a minimal number of two peptide ratios for a given protein ratio to be considered valid. For phosphopeptide analysis by MaxQuant, two options were added to the settings: variable modification of phosphorylation at $\mathrm{S}, \mathrm{T}$ and $\mathrm{Y}$, and dependent peptide search. Identified proteins were analyzed with Cytoscape software (GeneMania plugin) (61).

\section{Bimolecular fluorescent complementation (BiFC)}

We modified BIFC yeast plasmids (62) by inserting a mCH-VenusC reading frame in yeast plasmid for endogenous tagging pKT127 (57). VenusN fragment was cloned into pDK-HC plasmid for endogenous tagging with subsequent subcloning of $P A B$ 1-IFP or IFP to extend the ORF resulting in plasmids pDK-HC-VenusN-IFP/pDK-HC-VenusN-PAB1-IFP. To detect complementation signal yeast were grown to mid log phase in SD media containing $10 \mu \mathrm{M} \mathrm{Cu}^{2+}$, then cells were divided into samples grown in control and starvation conditions. After that cells were plated and visualized.

\section{Auxin-inducible degradation}

We modified pKT127 (57) plasmid by introducing AID-HPH fragment for endogenous tagging (63). AID-ymRFP pOQR4 plasmid was a kind gift from Michael Lisby. pBabe Blast osTIR19Myc was a gift from Andrew Holland (Addgene plasmid \#80073). We integrated OsTIR on pDKHC plasmid. Pkcl was endogenously tagged with AID or AID-ymRFP. Yeast cells were grown in SD media to middle log phase, $\mathrm{Cu}^{+}$was added to a $10 \mathrm{uM}$ final concentration to induce OsTIR 
expression. 1-naphthaleneacetic acid (NAA, Sigma) 10 $\mu \mathrm{M}$ or vehicle were added for 3 hours to induce Pkc1 depletion. The cells were then shifted to media with or without glucose for 1hour to induce SG formation.

\section{Microscopy}

For imaging yeast cells were grown to mid-log phase and seeded on concanavalin A (Sigma) coated 4-well microscope plates (IBIDI). Confocal 3D images and movies were acquired using a dual point-scanning Nikon A1R-si microscope equipped with a PInano Piezo stage (MCL), using a 60x PlanApo VC oil objective NA 1.40. Movies were acquired in resonant-scanning mode. Image processing was performed using NIS-Elements software. For super resolution Structured Illumination Microscopy (SIM) Cells were prepared as described above. Images were acquired using a Nikon nSIM microscope with a 488nm and 561nm lasers. A 100x oil TIRF objective (NA 1.49) was used for the imaging. Prior to imaging the point-spread function was visualized with 100

$\mathrm{nm}$ fluorescence beads in order to adjust the correction ring of the objective to the coverslip thickness. The final image was reconstructed using NIS-Elements software (Nikon). For super resolution Total Internal Reflection Microscopy cells were prepared as described above. Images were acquired using Nikon nSIM microscope equipped with TIRF module and a 100× Apochromat TIRF oil objective (NA 1.49) in 2D mode. The final image was reconstructed using NIS-Elements software.

\section{Western Blotting}

We used the following reagents to detect proteins: anti-GFP (ab290), anti-Glucose-6phosphatedehydrogenase antibody (Sigma A9521), anti-rabbit IgG (HRP) (ab6721, StreptavidinCy3, Streptavidin-HRP (Thermo Scientific).

\section{Protein sequence analysis}

Identification of Intrinsically Disordered Regions (IDRs) and Low Complexity (LC) regions Prediction of LC regions were performed using SEG software (64). Prediction of IDR was done usinf IUPred resource (65). 


\section{Statistics}

Three or more independent experiments were performed to obtain the data. $\mathrm{P}$ values were calculated by two-tailed Student t-test, or one-way ANOVA for samples with $\mathrm{N}>10$ following normal distribution. Normal distribution of the data was verified using Shapiro Wilk test and the equality of variances was verified by Levene's test. Mann Whitney, or Kruskal Wallis tests were used for experiments with less than 5 samples. The sample sizes were not predetermined.

Table S1. S. cerevisiae strains used in this study.

\begin{tabular}{|c|c|}
\hline Source & Genotype \\
\hline BY4741 & MATa his3 31 leu $2 \Delta 0$ met15 $5 \Delta 0$ ura $3 \Delta 0$ \\
\hline W303a & MATa leu2-3,112 trp1-1 can1-100 ura3-1 ade2-1 his3-11,15 \\
\hline This study & BY4741 PAB1-BIRA(R118G)-GFP-HPH \\
\hline This study & BY4741 PUB1-BIRA(R118G)-GFP-HPH \\
\hline This study & BY4741 PAB1-GFP-HPH PIL1-mCherry-KanMX \\
\hline This study & BY4741 PUB1-BIRA(R118G)-GFP-HPH \\
\hline This study & BY4741 PIL1-mCherry-VenusC pDK-HC-VenusN-PAB1-IFP \\
\hline This study & BY4741 PIL1-mCherry-VenusC $p D K-H C$-VenusN-IFP \\
\hline This study & BY4741 PAB1-mCherry-VenusC $p D K-H C-V e n u s N-P A B 1-I F P$ \\
\hline This study & BY4741 PAB1-mCherry-VenusC $p D K-H C$-VenusN-IFP \\
\hline This study & BY4741 pillA::KanMX $\triangle l s p 1: \because U R A 3$ PAB1-GFP-HPH \\
\hline This study & BY4741 PAB1-GFP-HPH \\
\hline This study & BY4741 PAB1-GFP-HPH $p D K-H C$ \\
\hline This study & BY4741 PAB1-GFP-HPH pDK-HC-PIL1-mCherry \\
\hline This study & BY4741 PIL1-GFP-KanMX \\
\hline This study & $\begin{array}{l}\text { BY4741 pil14::KanMX lsp14::URA3 PAB1-GFP-HPH pDK- } \\
\text { HC-PIL1 }\end{array}$ \\
\hline This study & $\begin{array}{l}\text { BY4741 pil14::KanMX lsp1A::URA3 PAB1-GFP-HPH pDK- } \\
\text { HC-PIL1(1-276) }\end{array}$ \\
\hline
\end{tabular}




\begin{tabular}{|c|c|}
\hline This study & $\begin{array}{l}\text { BY4741 pilla::KanMX lsp14::URA3 PAB1-GFP-HPH pDK- } \\
\text { HC-PIL1S230A }\end{array}$ \\
\hline This study & $\begin{array}{l}\text { BY4741 pilla::KanMX lsp14::URA3 PAB1-GFP-HPH pDK- } \\
\text { HC-PIL1S230D }\end{array}$ \\
\hline This study & $\begin{array}{l}\text { BY4741 pil14::KanMX lsp14::URA3 PAB1-GFP-HPH pDK- } \\
\text { HC-PIL1S163A }\end{array}$ \\
\hline This study & $\begin{array}{l}\text { BY4741 pilla :KanMX lsp14::URA3 PAB1-GFP-HPH pDK- } \\
\text { HC-PIL1S163D }\end{array}$ \\
\hline This study & W303 pDK-AC-OsTIR PKC1-AID-myRFP-KanMX \\
\hline This study & W303 pDK-AC-OsTIR PKC1-AID-KanMX PIL1-GFP-HPH \\
\hline This study & W303 pDK-AC-OsTIR PKC1-AID-KanMX PAB1-GFP-HPH \\
\hline This study & BY4741 pDK-HC-PKC1-mCherry \\
\hline This study & BY4741 pDK-HC-PKC1R398P-mCherry \\
\hline This study & BY4741 pill4::KanMX lsp14::URA3 pDK-HC-PKC1-mCherry \\
\hline This study & $\begin{array}{l}\text { BY4741 pilla::KanMX lsp14::URA3 pDK-HC-PKC1R398P- } \\
\text { mCherry }\end{array}$ \\
\hline This study & BY4741 pDK-HC-Ruby2-PKC1-Clover \\
\hline This study & BY4741 pDK-HC-Ruby2-Clover \\
\hline This study & BY4741 PIL1-DDR2-KanMX \\
\hline This study & BY4741 PIL1-GFP-KanMX pDK-HC-PKC1T983A-mCH \\
\hline This study & BY4741 PAB1-GFP-KanMX pDK-HC-PKC1T983A-mCH \\
\hline This study & BY4741 pDK-HC-OsTIR PKCl-AID::HPH pDK-HC-PIL1-mCH \\
\hline This study & $\begin{array}{l}\text { BY4741 pDK-HC-OsTIR } \quad \text { PKC1-AID::HPH } \quad \text { pDK-HC- } \\
\text { PIL1S163D-mCH }\end{array}$ \\
\hline This study & $\begin{array}{l}\text { BY4741 pDK-HC-OSTIR } P K C 1 \text {-AID::HPH } \quad \text { pDK-HC- } \\
\text { PIL1S230D-mCH }\end{array}$ \\
\hline This study & BY4741 slt2 $\triangle::$ KanMX PAB1-GFP::HPH \\
\hline
\end{tabular}


Table S2. Plasmids used in this study.

\begin{tabular}{|c|c|}
\hline Plasmid & Source \\
\hline pKT127-BIRAR118G-GFP-HPH & This study \\
\hline pKT127-mCherry-VenusC & This study \\
\hline pDK-HC-VenusN-PAB1-IFP & This study \\
\hline pDK-HC-VenusN-IFP & This study \\
\hline pDK-HC-PIL1-mCherry & This study \\
\hline pDK-HC-PIL1(1-276)-mCherry & This study \\
\hline pDK-HC-PIL1S230A-mCherry & This study \\
\hline pDK-HC-PIL1S230D-mCherry & This study \\
\hline pDK-HC-PIL1S163A-mCherry & This study \\
\hline pDK-HC-PIL1S163D-mCherry & This study \\
\hline $\mathrm{pDK}-\mathrm{HC}-\mathrm{PKCl}$-mCherry & This study \\
\hline pKT127-AID-HPH & This study \\
\hline pDK-HC-PKC1R398P-mCherry & This study \\
\hline pDK-AC-OsTIR & This study \\
\hline pDK-HC-Ruby2-Clover & This study \\
\hline pDK-HC-Ruby2-PKC1-Clover & This study \\
\hline pDK-HC-PKC1T983A-mCH & This study \\
\hline
\end{tabular}

Acknowledgments: We thank Dr. William Breuer for proteomics data acquisition and Prof. Dr. Thomas Kneib for statistical consultation. Funding: This work was supported by the European Research Council under the European Union's Seventh Framework Programme (FP/20072013)/ERC-StG2013 337713 DarkSide starting grant and a EU Joint Programme Neurodegenerative Disease Research (JPND) 2 grant. TA was funded by the Jerusalem Brain Community Doctoral Fellowship for the part of this project. The authors declare no conflicts of interest. Author contributions: All aspects of the work comprising the manuscript were carried out jointly by DK and TA. Competing interests: The authors declare that they have no competing 
interests. Data and materials availability: The mass spectrometry proteomics data have been deposited to the ProteomeXchange, https:/www.ebi.ac.uk/pride (66) with the datasets identifiers PXD016988 and PXD017502. 


\section{Figure Legends}

Fig. 1. Stress granules associate with eisosomes during starvation. (A) Strategy for BioID proteomics analysis of yeast proteins adjacent to Pab1 and Pub1 after starvation but not Pab1- or Pub1-adjacent without starvation. (B and C) Gene ontology (GO) analysis of SG proteins significantly enriched in Pab1 and Pub1 starvation interactomes hierarchically arranged (B) and eisosome components identified in starvation conditions (C). $\mathrm{N}=3$ biological replicates. (D) Confocal microscopy of SGs and eisosomes in yeast expressing endogenously tagged Pill-mCH and Pab1-GFP that were starved for $30 \mathrm{~min}$. Arrowheads indicate SGs. Images and kymograph are representative of 4 independent experiments. Quantification of the ratio of SGs proximal to eisosomes is noted in the merged images and represent the means $\pm \mathrm{SEM}$ of 100 cells pooled from all experiments (see also fig. S1I). (E) BiFC analysis of Pab1 and Pill proximity in cells expressing endogenously tagged Pill-mCH-VenusC (Vc) and inducible VenusN-Pab1-IFP (Vn) that were starved for $1 \mathrm{~h}$. Images are representative of 3 independent experiments. Fold change of fluorescence intensity are means \pm SEM of 30 cells per strain pooled from all experiments, * $\mathrm{p}<0.05$ by Student $\mathrm{t}$ test. (F) Live cell SIM of SG and eisosomes during starvation. Images are representative of 4 independent experiments. (G) Distances between eisosomes and SGs as measured by SIM are indicated as mean \pm SEM of 30 pairs pooled from all experiments, * $-\mathrm{p}<0.05$ by Student $\mathrm{t}$ test. (H) SG formation in WT and pil1s lsp $1 \Delta$ strains under starvation conditions. Images are representative of 3 independent experiments. (I) SG formation in pill $1 \Delta l s p 1 \Delta$ (control) and pills $l s p 1 \Delta$ complemented with Pill-mCH strains in starvation conditions. Images are representative of 3 independent experiments. (J) Western blot for endogenous Pab1 tagged with GFP in lysates of WT and pills $l s p 1 \Delta$ yeast. The numbers below the blot represent combined densitometry results from 3 independent experiments normalized to WT control, $\mathrm{p}=0.17$ by Kruskal Wallis test. Scale bars, $1 \mu \mathrm{m}$ (D, E, F, H, I) and $200 \mathrm{~nm}$ (inset in F).

Fig. 2. Pil1 undergoes Pkc1-dependent rearrangements during starvation. (A) Confocal and TIRF/SIM microscopy of eisosomes in live yeast expressing endogenously tagged Pil1-GFP and starved for $1 \mathrm{~h}$ Images are representative of 3 independent experiments. Imaging plane is indicated above the frames. (B) Quantification of the distance between eisosomes on a radial cell section is shown as a mean $\pm \mathrm{SEM}, \mathrm{p}<0.05$. Student $\mathrm{t}$ test, $\mathrm{n}=100$ cells per condition in 3 independent 
experiments. (C) Western blot for endogenous GFP-tagged Pill in lysates of WT yeast under control and $1 \mathrm{~h}$ starvation conditions. The numbers below the blot represent densitometry combined from 3 independent experiments, $p=0.61$ by Mann Whitney test. (D) Schematic of Pill and Pab1 showing protein domains, low-complexity regions (LCR), and intrinsically disordered regions (IDR). Phosphosites in Pill identified during starvation conditions ( $\operatorname{Ser}^{163}$ and $\mathrm{Ser}^{230}$ ) are indicated. (E) Confocal microscopy in live pills $l s p 1 \Delta$ yeast expressing endogenously tagged Pab1-GFP and induced to express mCH-tagged WT, S230A, S230D, S163A, S163D, or truncated (1-276) Pill before the onset of starvation. Images are representative of 3 independent experiments. (F) Quantification of the ratio of cells in the population with SGs and the distance between the eisosomes. Data represent means \pm SEM of 100 cells per strain pooled from all experiments for distance quantification and 1000 cells per strain pooled from all experiments for ratio quantification, $\mathrm{N}=20$. Pearson correlation between $\mathrm{SG}$ ratio and the distance between eisosomes $=-0.74, *$ - indicates significant differences in the distance or ratio of cells with SGs comparing to WT, *- p $<0.05$ with one-way Anova. (G) Experimental scheme for assessing eisosome formation in yeast expressing endogenously tagged Pkc1-AID, an integrated copy of the auxin receptor OsTIR (51), and endogenously tagged Pill-GFP. Yeast were grown to middle log phase, then Pkcl depletion was initiated by addition of a synthetic auxin analogue before cells were starved and visualized. (H) Confocal imaging and quantification of the distance between eisosomes in of cells treated as in $(G)$. Data are mean \pm SEM of 30 cells per group per condition pooled from 3 independent experiments. Scale bars, $1 \mu \mathrm{m}$ (A, E top row, H), $500 \mathrm{~nm}$ (E bottom row), and $100 \mathrm{~nm}$ (insets in A).

Fig. 3. Pkc1 localizes to stress granules and is required for stress granule formation. (A) Schematic of Pkc1 showing protein domains, low complexity regions (LCR), and intrinsically disordered regions (IDR). The R398P mutation renders Pkc1 constitutively active (31). The T983A mutation blocks Pkc1 phosphorylation by Pkh2. (B) Live confocal microscopy of yeast expressing endogenously tagged Pab1-GFP and either inducible WT or constitutively active (Pkc1-R398P) Pkc1-mCH grown in control or starvation conditions. Quantified portion of SGs with detectable $\mathrm{Pkc1}$ are noted in the merged images. Fluorescence intensity of Pkc1-mCH in SGs were quantified and presented as means \pm SEM from 30 cells per genotype per condition pooled from 3 independent 
experiments. ${ }^{* *}-\mathrm{p}<0.01$.(C) Quantified ratios of dead cells in the population of WT and pill $\Delta$ lspl $1 \Delta$ yeast overexpressing an integrated copy of Pkc1-mCH or Pkc1-R398P-mCH stained with propidium iodide. Data represent means \pm SEM of 100 cells per genotype per condition pooled from 3 independent experiments. ${ }^{*}$ - $\mathrm{p}<0.05$. (D) Confocal microscopy of SGs in pill $1 \Delta l s p 1 \Delta$ yeast overexpressing Pkc1 or Pkc1-R398P prior to the starvation and quantification of the ratios of cells in the population with SGs. Data represent means \pm SEM of 30 images containing at least 20 cells of each type from 3 independent experiments. (E) Confocal microscopy of yeast expressing endogenously tagged Pil1-GFP and induced to express PKC1(T983A)-mCH $1 \mathrm{~h}$ before starvation. Images are representative of 3 independent experiments. (F) Schematic of FRET sensor for Pkc1 activity and control. Ruby2 (red, acceptor) and Clover (green, donor) are fused to opposite ends of $\mathrm{Pkc1}$. The control is a head-to-tail fusion of Ruby2 and Clover. Activation of Pkc1 is thought involve a change from a closed to an open conformation (35), thus reducing the FRET signal. (G) Confocal microscopy showing Pkc1 activity as assessed by FRET in WT yeast grown in control or starvation conditions or in the presence of the Pkc1-activating compound capsofungin. (I) Quantification of FRET activity in (H). Data represent means \pm SEM of 30 cells per condition pooled from 3 independent experiments, * - p<0.05, with one-way Anova (H) Quantification of donor (Clover)/acceptor (Ruby2) fluorescence intensities inside cells during control and starvation conditions. Graphs are representative of 10 independent experiments. (J) Model of eisosome rearrangement during starvation. Pkc1-dependent phosphorylation of eisosomes during starvation results in their clustering, which primes formation of SGs that recruit active Pkc1. Scale bars, 1 $\mu \mathrm{m}$.

Fig. 4. Stress Granules promote stress recovery by storing Pkc1 (A) Experimental scheme and confocal microscopy of middle log phase yeast induced to express Pkc1-mCH for $2 \mathrm{~h}$, then starved for $24 \mathrm{~h}$ before being placed in fresh glucose-containing media for $2 \mathrm{~h}$. (B) Quantification of whole-cell fluorescence intensities from (A). Data represent means $\pm \mathrm{SEM}$ of 30 cells per genotype per condition pooled from 3 independent experiments. * $-p<0.05$ (C) Growth curves of WT and pills lsp $1 \Delta$ yeast that were grown in control conditions to middle log phase or for 24 hours in stationary phase, then diluted in fresh glucose-containing media. Graphs are representative of 3 independent experiments. Data represent means \pm SEM. (D) Model of WT and pill $l s p 1 \Delta$ 
recovery after long-term starvation, showing that absence of SGs results in delayed resumption of division. Scale bars, $1 \mu \mathrm{m}$.

Fig. S1. Stress granules associate with eisosomes during starvation. (A) Confocal microscopy showing the indicated proteins in yeast expressing endogenous Pab1 or Pub1 tagged with BirA(R118G)-GFP grown without biotin, with excess biotin, or with biotin but without glucose for 6 h. Images are representative of 3 independent experiments. (B) Fluorescence intensity (FI) profile for green (Pab1-GFP) and red (Cy3-streptavidin) channels in the +Biotin starvation condition in (A), measured where indicated with the white line in the merged image. Data are representative of 3 independent experiments. (C) Western blot for biotinylated proteins and GFP in PAB1-BirA(R118G)-GFP yeast grown with biotin under starvation conditions. Blots are representative of 3 independent experiments. (D) Live cell SIM of SGs (Pab1-GFP, green) and eisosomes (Pil1-mCH, red) during starvation. Images are representative of 3 independent experiments. (E to $\mathbf{G})$ Confocal microscopy showing endogenously tagged Pab1-mCH colocalization with Pub1-GFP and Pbp1-GFP (E), Lsp1-mCH and endogenously tagged Pab1-GFP (F), or Pil1-mCH and endogenously tagged Pab1-GFP (G) in stationary phase or after starvation for 1 hour as indicated. Images are representative of 3 independent experiments. Arrowheads indicate SGs. (H to $\mathbf{J})$ Confocal microscopy of eisosomes and SGs in yeast expressing endogenous Pab1 tagged with GFP and Pill tagged with $\mathrm{mCH}$ grown to middle log phase and starved for the indicated amounts of time. The same cells were tracked in the start of starvation $(\mathrm{H})$ and after $1 \mathrm{~h}$ of starvation $(\mathrm{J})$ for the indicated amounts of time. Images and FI profiles for green (Pab1-GFP) and red $(\mathrm{Pil1}-\mathrm{mCH})$ channels are representative of 3 independent experiments. Quantified ratios of proximal SGs during the course of starvation (I) are means \pm SEM of 100 cells per condition pooled from all experiments. (K) Live cell SIM of SGs (Pab1-mCH) and actin bodies (LifeActGFP $(25,52))$ during starvation. Images are representative of 3 independent experiments. (L) BiFC analysis of Pab1 in cells expressing endogenously tagged Pab1-mCH-VenusC (Vc) and inducible VenusN-Pab1-IFP (Vn) starved for $1 \mathrm{~h}$. Fold change in FI are means \pm SEM of 30 cells from 3 independent experiments. (M) FI of SGs in WT and pills $l s p 1 \Delta$ yeast. Data represent means \pm SEM of 100 SGs from 10 independent experiments. Scale bars, $1 \mu \mathrm{m}$. 
Fig. S2. Eisosomes undergo Pkc1-dependent clustering during starvation. (A) TIRF-SIM microscopy of eisosomes in yeast expressing Pill endogenously tagged with mCH starved for $1 \mathrm{~h}$. Images are representative of 3 independent experiments. (B) Confocal microscopy and fluorescence intensity (FI) profile of eisosomes in yeast expressing Pill endogenously tagged with GFP grown in normal conditions. Image and data are representative of 3 independent experiments. (C) Serial dilutions of pill $\Delta l s p 1 \Delta$; Pab1-GFP yeast complemented with a single copy of inducible WT, S230A, S230D, S163A, S163D, or truncated (1-276) forms of Pil1. Images are representative of 3 independent experiments. (D) Confocal microscopy of yeast overexpressing an integrated copy of Pill-mCH and inducible Pab1-GFP. Arrowhead indicates a cytoplasmic fibril. Images are representative of 3 independent experiments. (E) Confocal microscopy of eisosomes (red) and bud scars in yeast expressing Pill endogenously tagged with $\mathrm{mCH}$ and stained with Calcofluor White. Quantified average distances between eisosomes in young (0-4 scars) and old (more than 4 scars) cells are means \pm SEM of 30 cells from 3 independent experiments. (F) Confocal microscopy of $\mathrm{Pkcl}$ in yeast expressing Pkcl endogenously tagged with AID-ymRFP, an integrated copy of the auxin receptor OsTIR (51), and endogenously tagged Pill-GFP that were grown to middle log phase before Pkcl depletion was initiated by addition of a synthetic auxin analogue NAA for $2 \mathrm{~h}$. Quantified whole-cell FI of Pkc1-AID-ymRFP are means \pm SEM of 30 cells from 3 independent experiments. (G) Confocal microscopy of untagged Pkc1 and Pkc1-AID in yeast cells stained with propidium iodide to indicate live cells. Ratios of dead cells in the population are mean \pm SEM of 100 cells pooled from 3 independent experiments. (H) Confocal microscopy of eisosomes in slt2 $\Delta$ yeast expressing Pill endogenously tagged with GFP grown in control and $1 \mathrm{~h}$ starvation conditions. Quantified distances between eisosomes are mean \pm SEM of 30 cells pooled from 3 independent experiments. ( $\mathbf{I}$ and $\mathbf{J}$ ) Confocal microscopy and quantification of the distance between eisosomes in live yeast expressing endogenously tagged Pkc1-AID, an integrated copy of OsTIR, and complemented with a single copy of inducible WT, S230D, or S163D forms of Pill that were grown to middle log phase, treated with NAA for $2 \mathrm{~h}$, and starved for $1 \mathrm{~h}$. Data are means \pm SEM of 30 cells pooled from 3 independent experiments. Scale bars, 1 $\mu \mathrm{m}$. 
Fig. S3. Pkc1 localizes to stress granules during starvation. (A) Confocal microscopy of the indicated proteins in yeast expressing endogenously GFP-tagged Pkc1 and Pab1 and grown in control and $1 \mathrm{~h}$ starvation conditions. Arrowhead indicates SGs. Images are representative of 3 independent experiments. (B) Confocal images of propidium iodide staining in WT and pills lspld yeast overexpressing WT Pkc1 or Pkc1 R398P tagged with $\mathrm{mCH}$ under normal conditions. Images are representative of 3 independent experiments. (C) Quantification of SGs per cell for yeast overexpressing Pkc1 or Pkc1 R398P tagged with $\mathrm{mCH}$ and starved for $1 \mathrm{~h}$. Data represent means \pm SEM of 100 SGs from 10 independent experiments. (D) Serial dilutions of WT and pil1s $l s p 1 \Delta$ strains grown in the presence of the indicated amounts of copper. (E) Quantification of donor/acceptor fluorescence intensity for yeast expressing Pkc1 sensor in caspofungin-treated (whole-cell average) or starvation (SG area) conditions. Data represent means \pm SEM of 30 cells from 3 independent experiments. (F) Photoconversion of eisosomes in yeast expressing endogenously tagged Pill-DDR2 in starvation conditions. Images are representative of 3 independent experiments. ( $\mathbf{G}$ to $\mathbf{J})$ Confocal microscopy of Pkc1 T983A in yeast expressing endogenously tagged Pab1-GFP, an integrated copy of Pkc1-T983A-mCH, and stained with Hoechst $(\mathrm{G})$ in control $(\mathrm{G})$ and starvation $(\mathrm{H}, \mathrm{I})$ conditions. Images are representative of 3 independent experiments. Scale bars, $1 \mu \mathrm{m}$. 


\section{Figure 1}

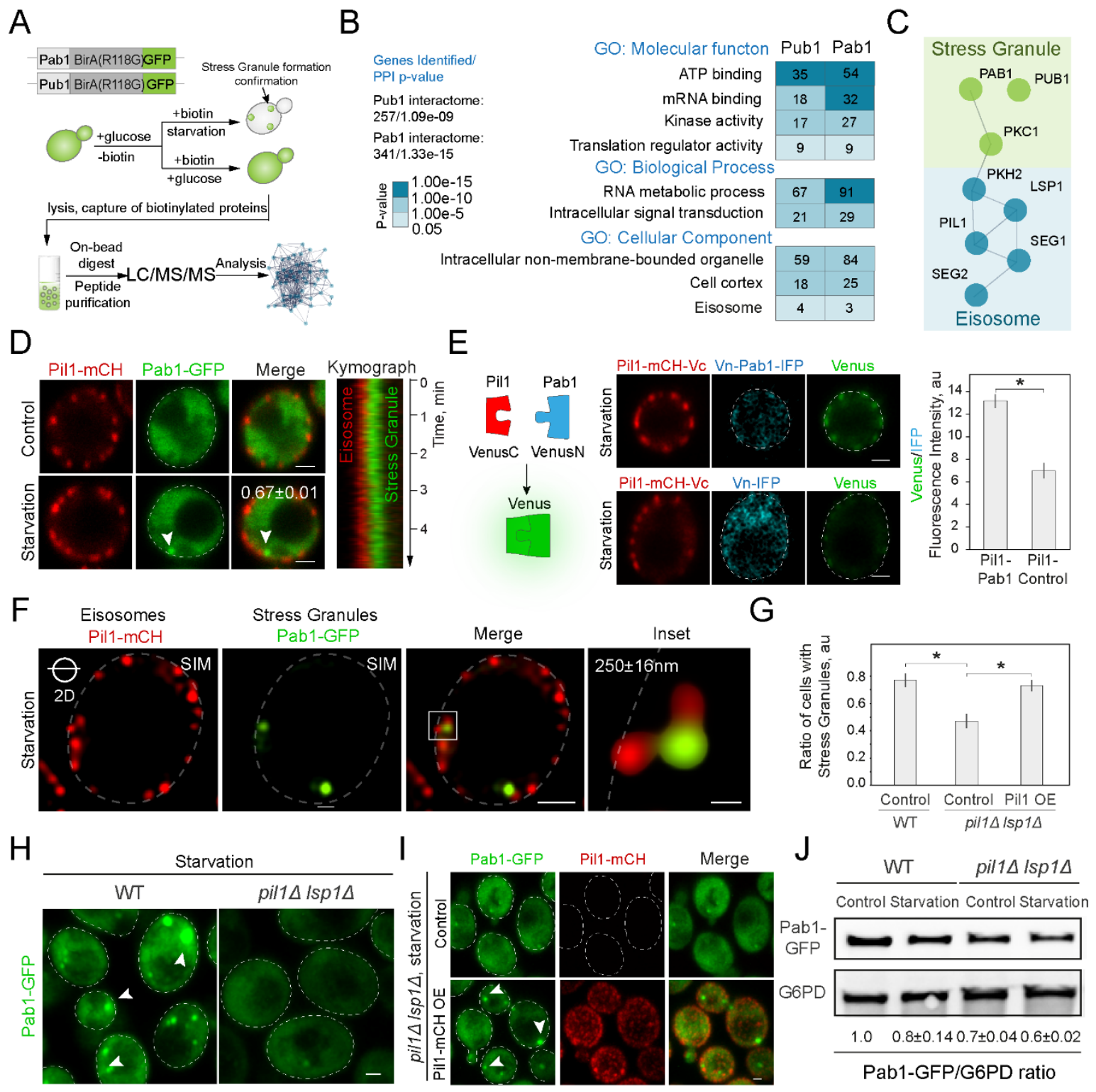




\section{Figure 2}
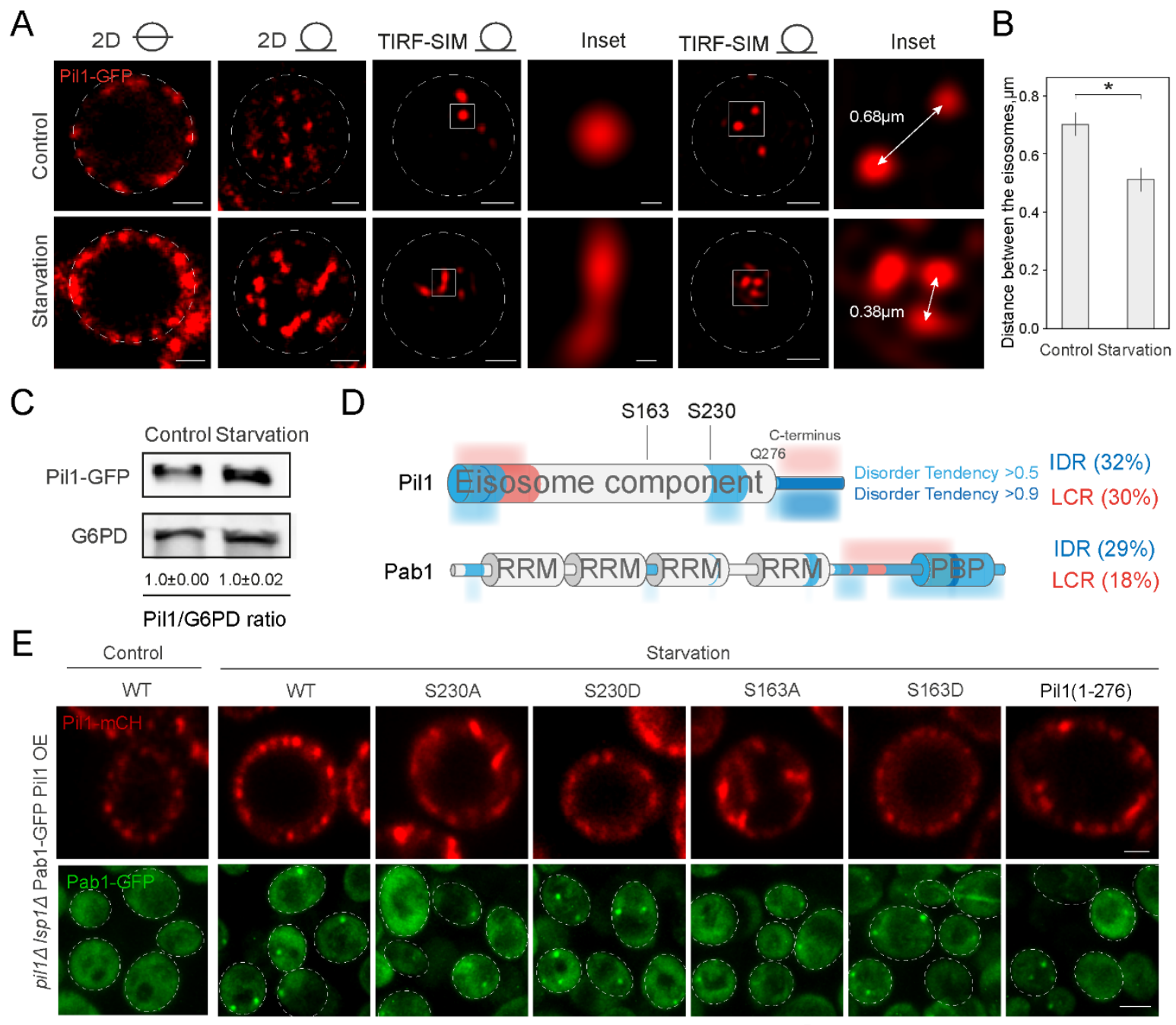

S163A

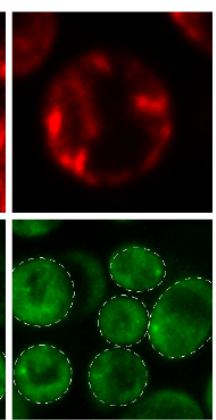

G

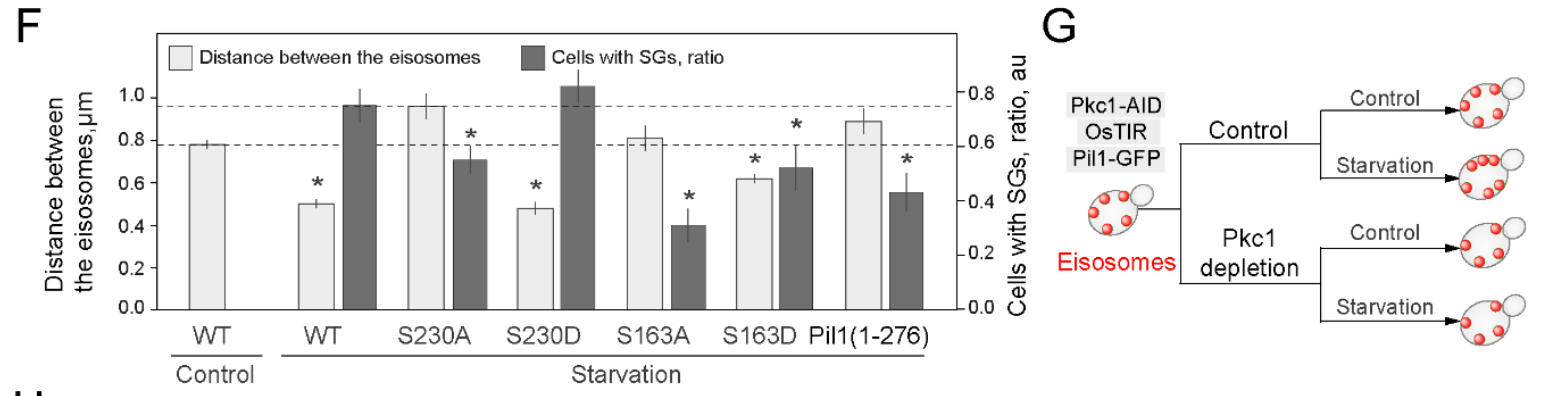

$\mathrm{H}$
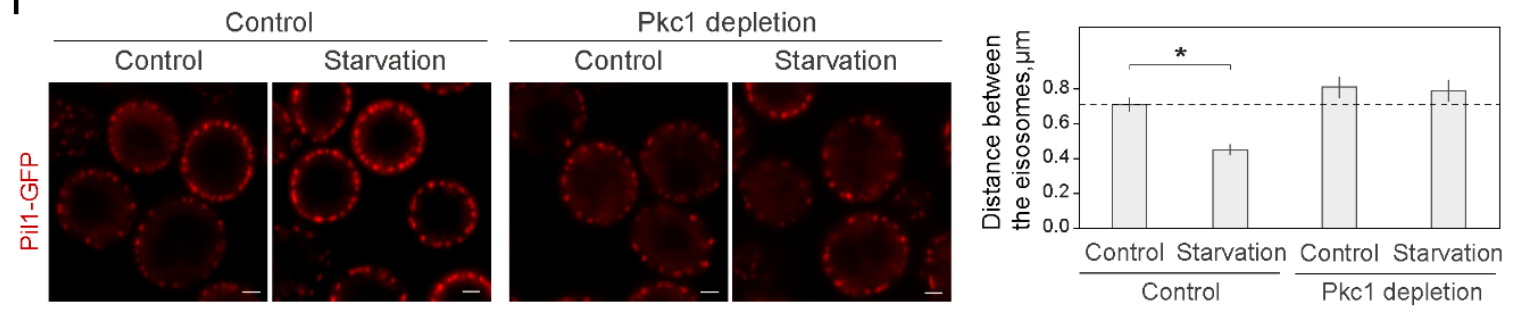


\section{Figure 3}
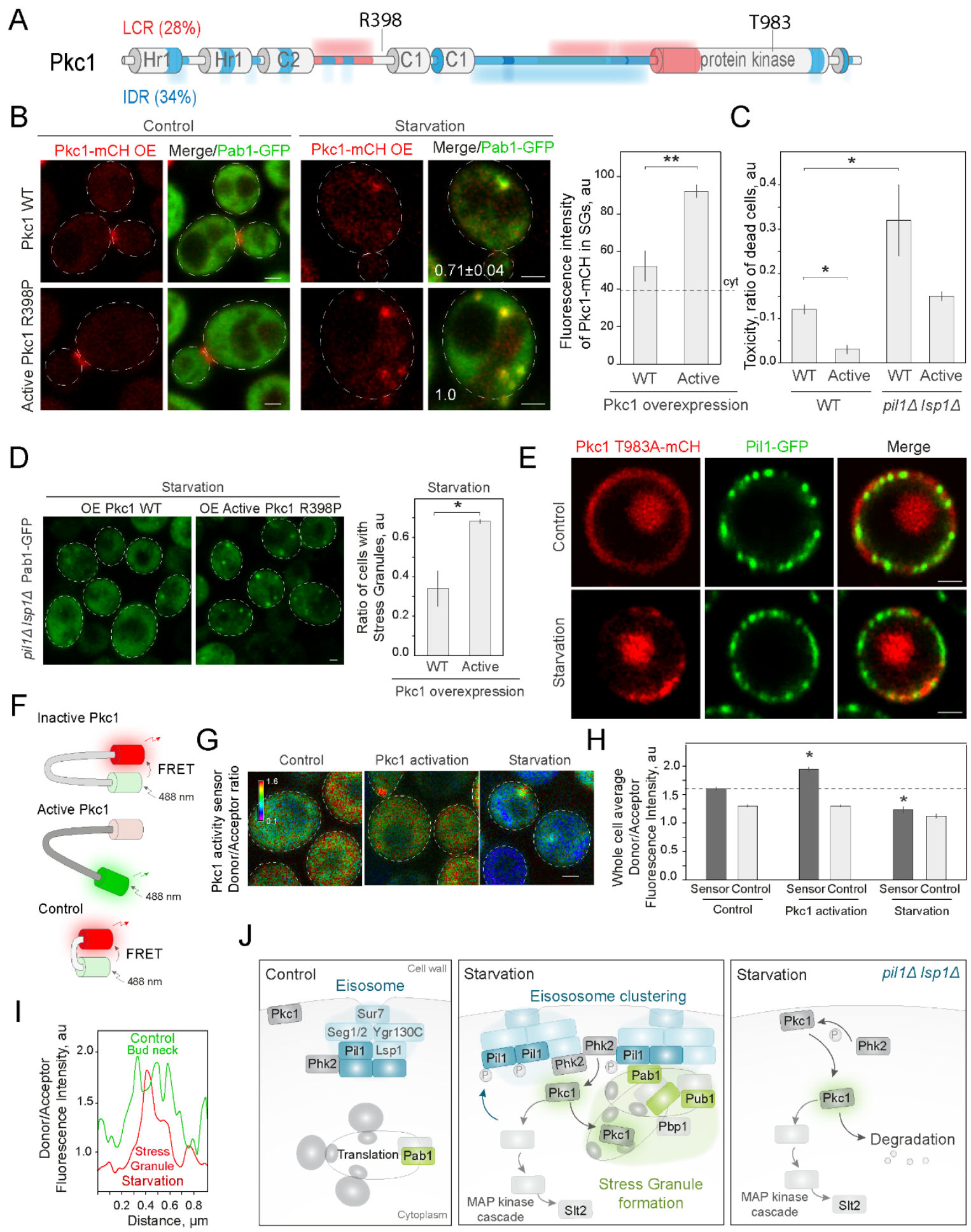
Figure 4

A
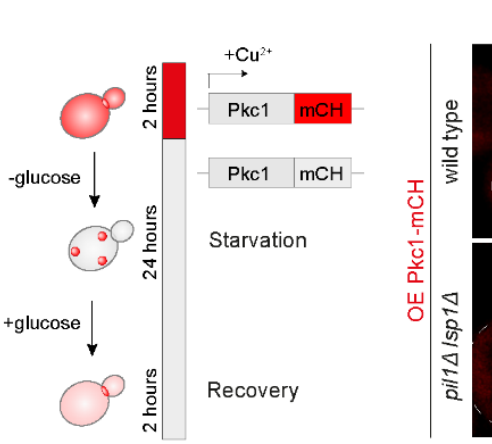

Control

Starvation
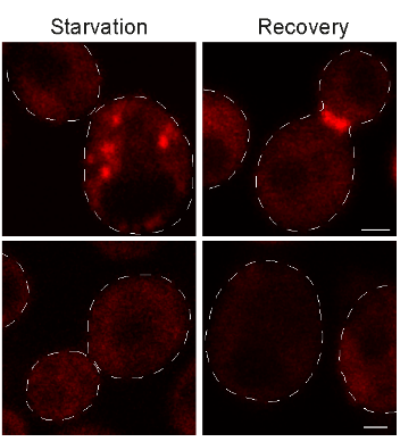

B

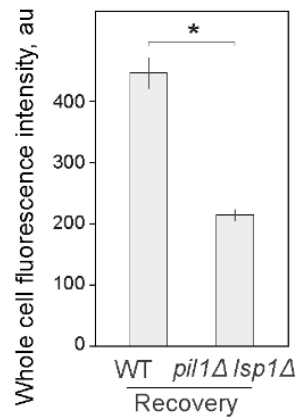

C

D

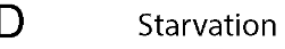

Recovery
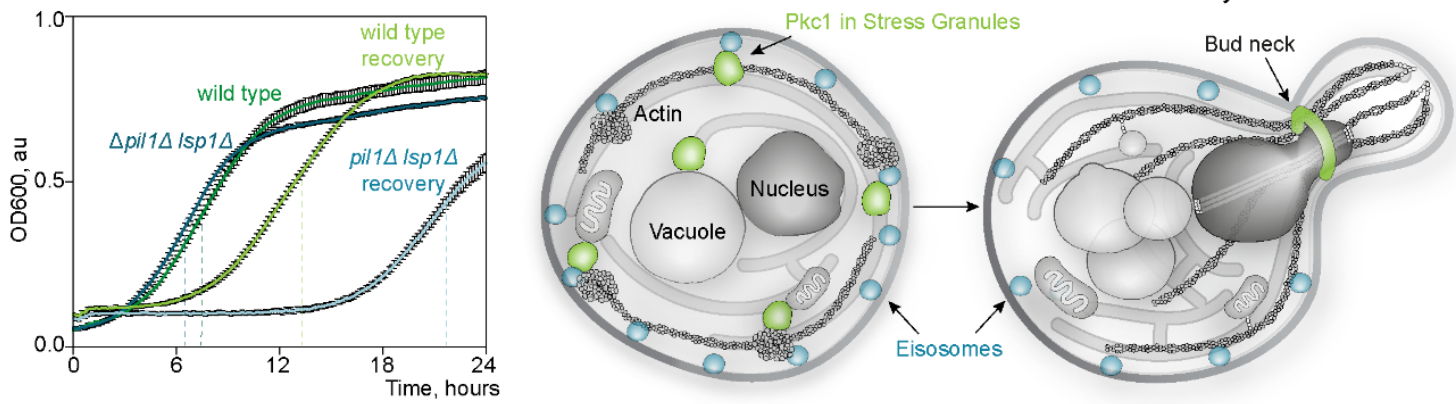
Figure S1.
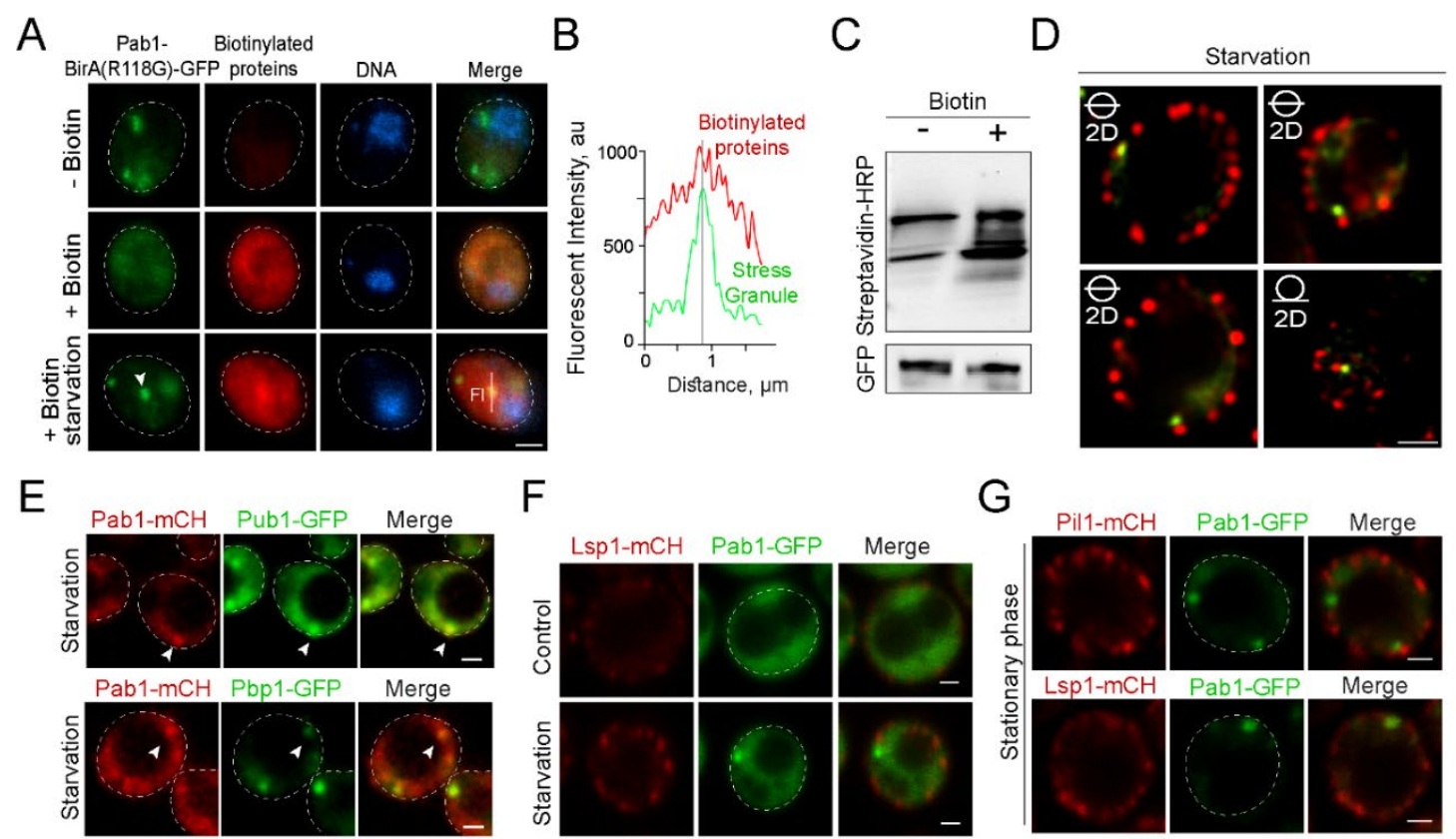

F

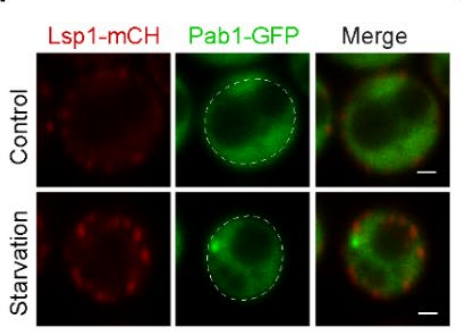

G

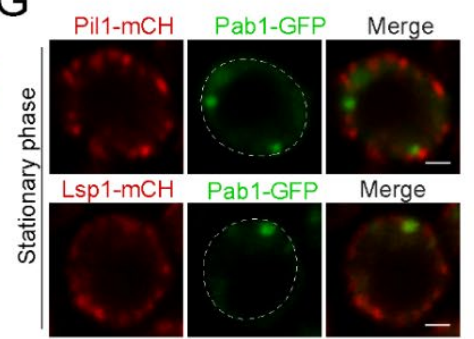

$\mathrm{H}$
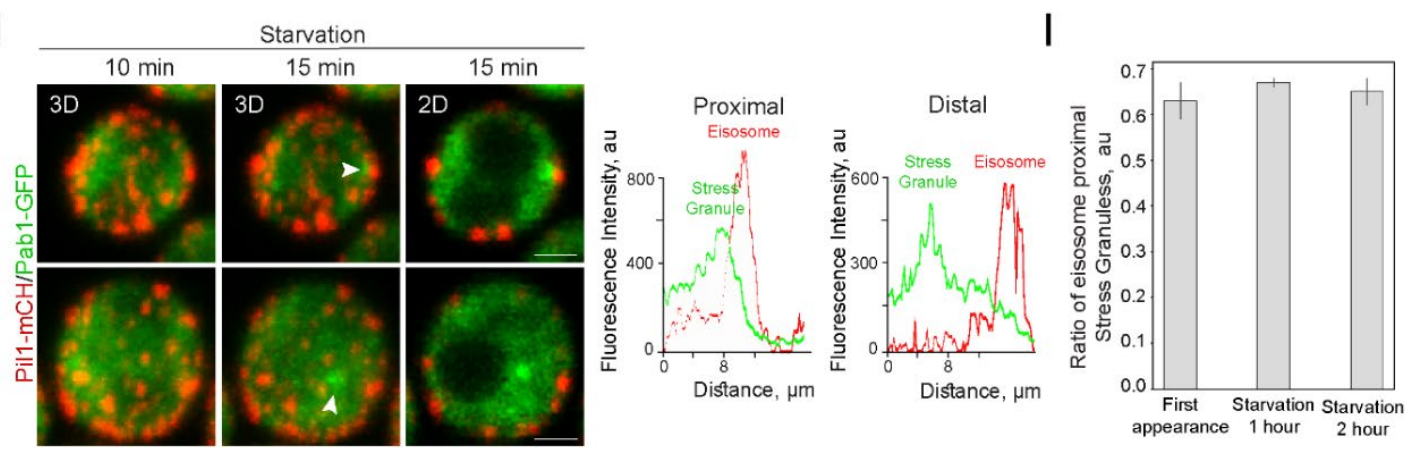

$\mathrm{J}$

Starvation

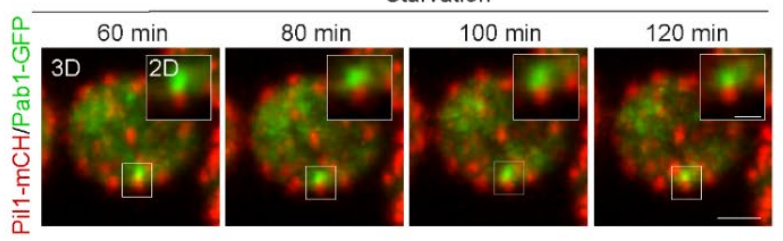

K Actin LifeAct-GFP Merge/Pab1-mCH

L
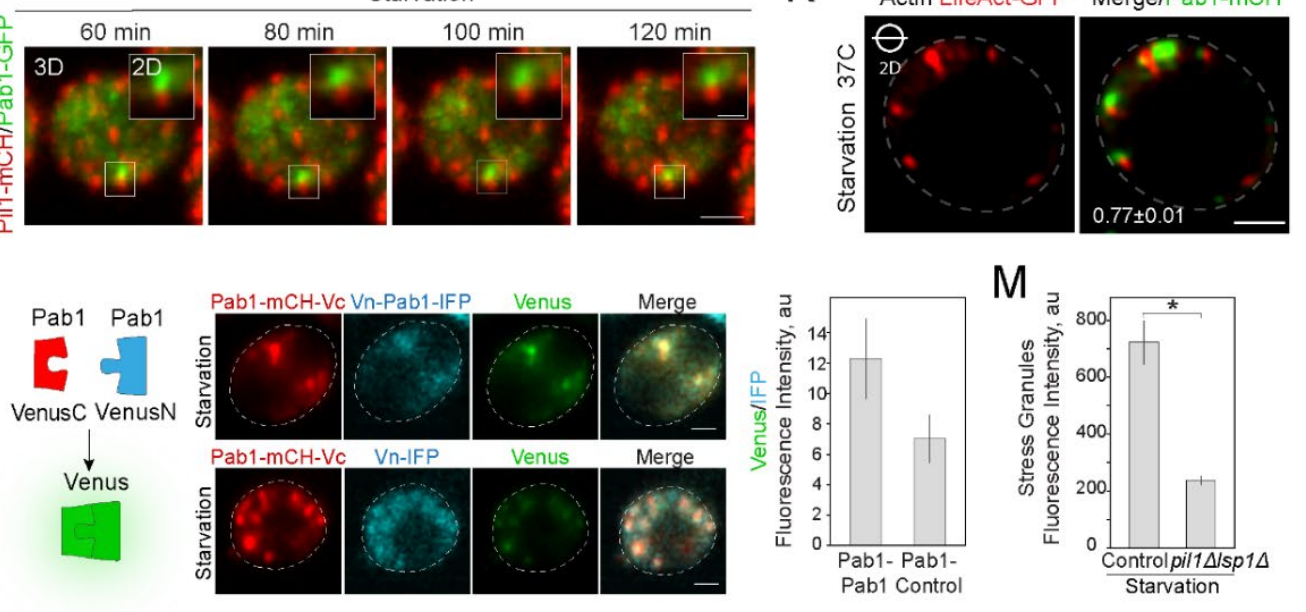


\section{Figure S2}
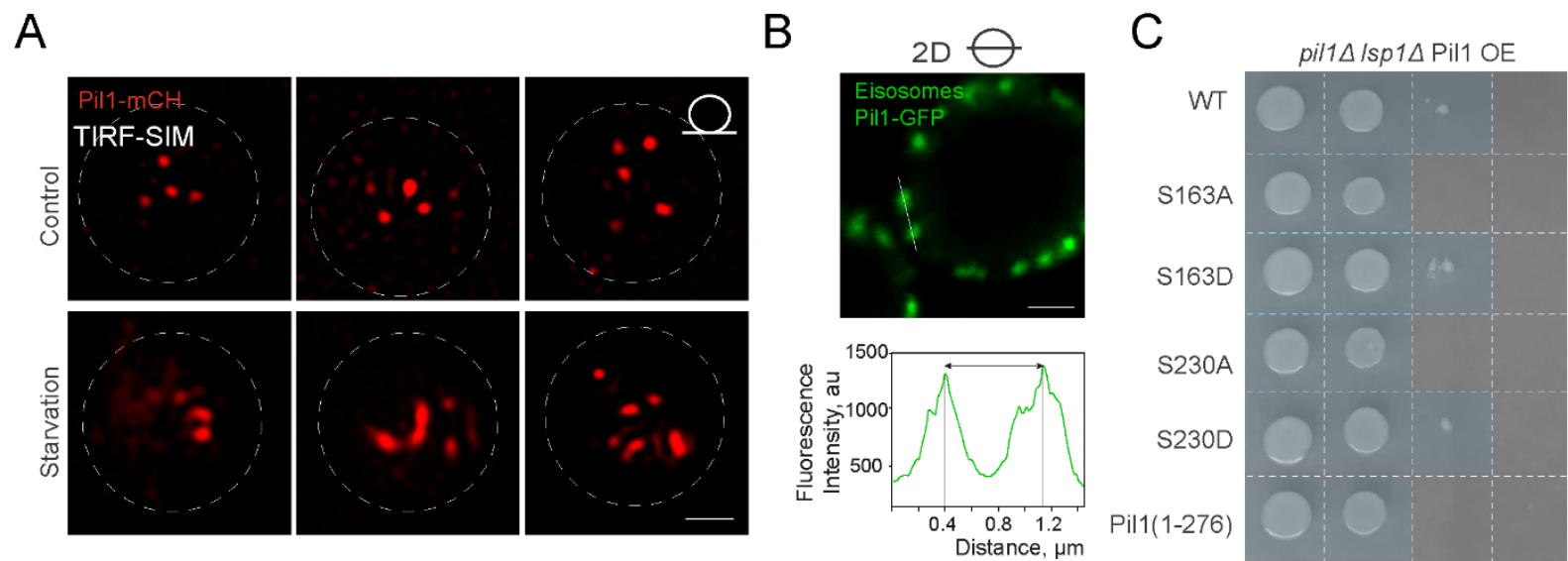

D

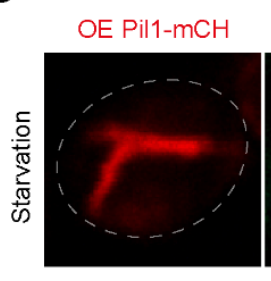

F

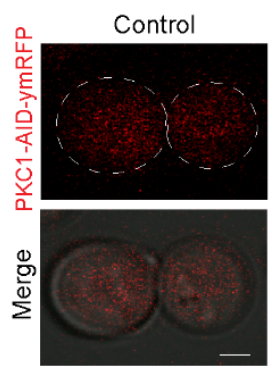

$\mathrm{H}$

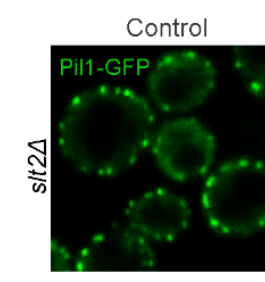

$\mathrm{J}$

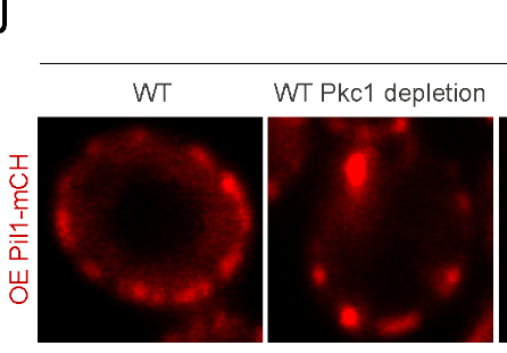

Pkc1 depletion
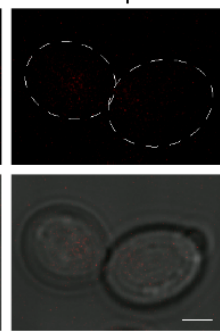

Starvation

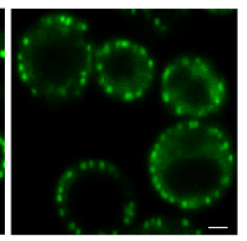

E
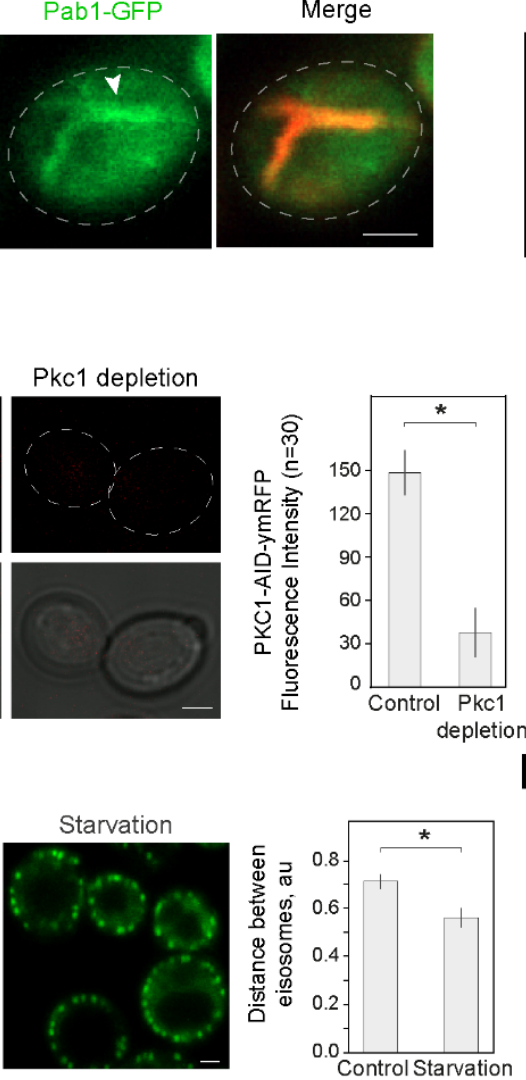

G
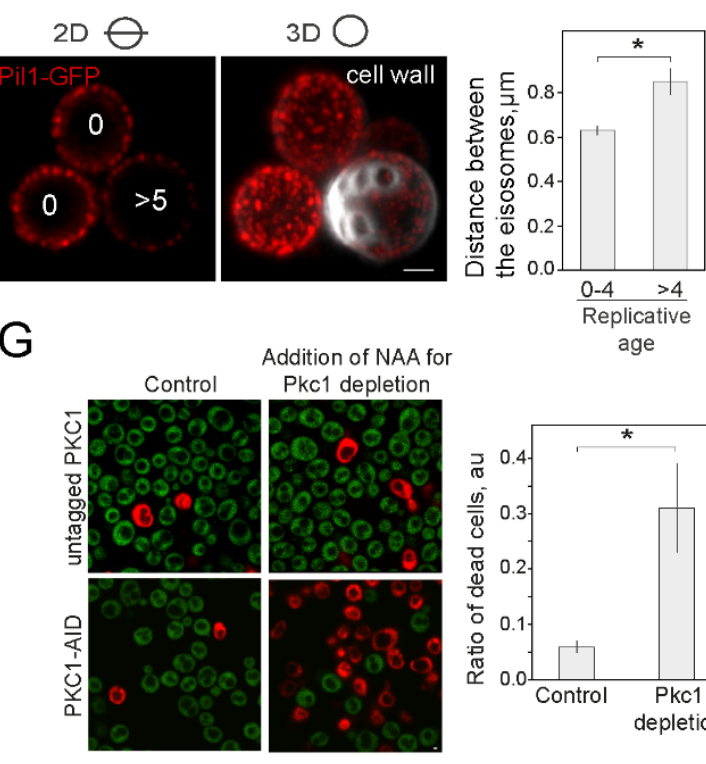

Addition of NAA for Pkc1 depletion
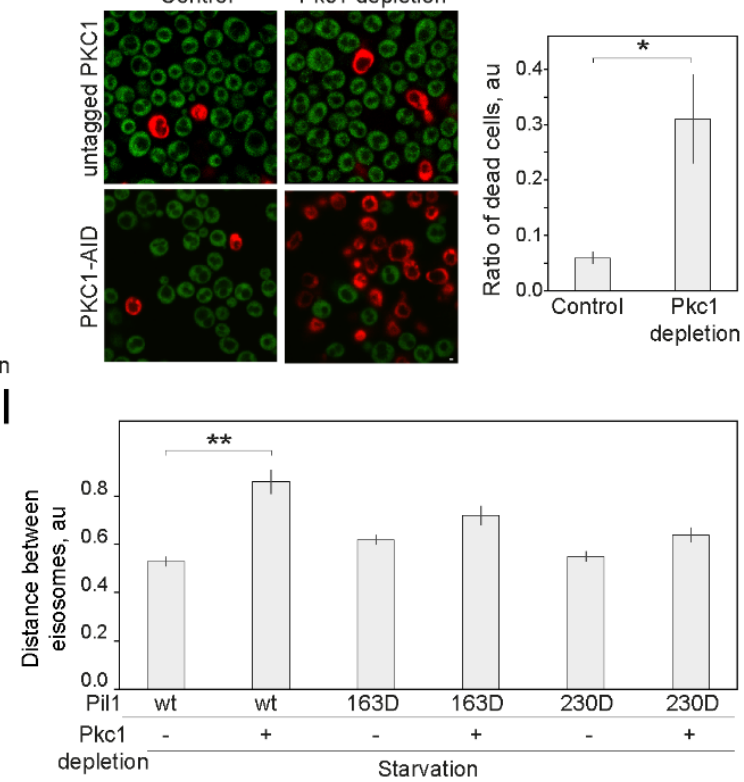

Starvation
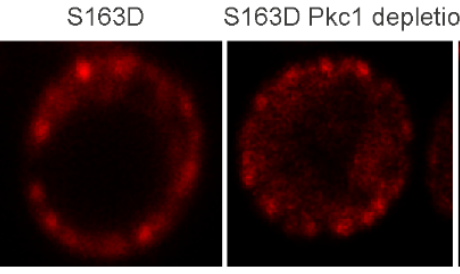

S230D

S230D Pkc1 depletion
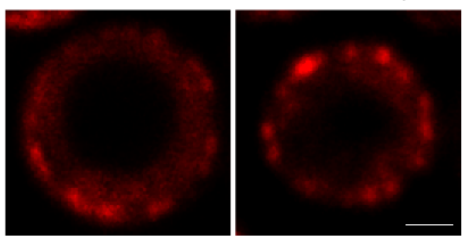
Figure S3

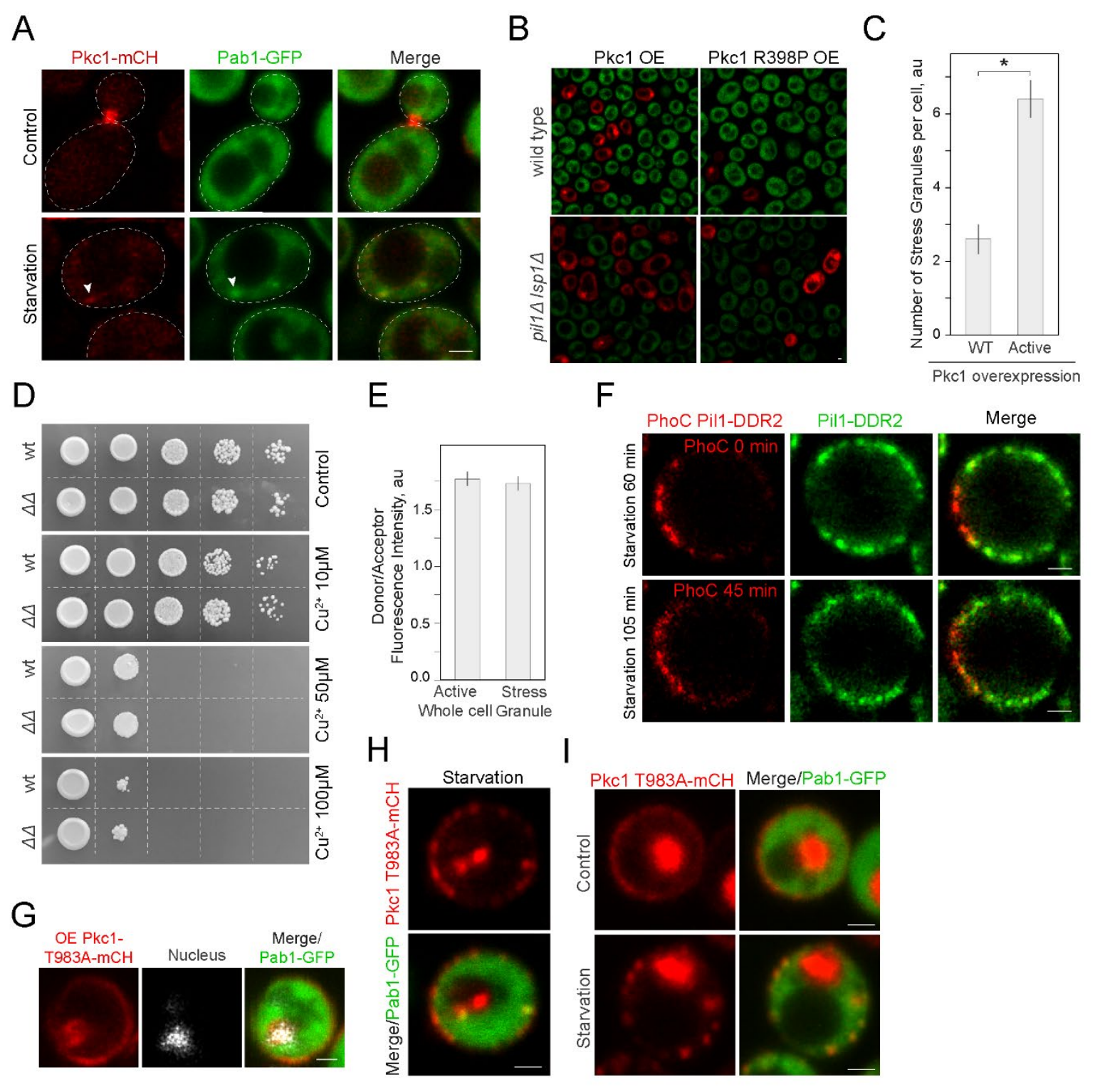




\section{References}

1. K. Kono, A. Al-Zain, L. Schroeder, M. Nakanishi, A. E. Ikui, Plasma membrane/cell wall perturbation activates a novel cell cycle checkpoint during G1 in Saccharomyces cerevisiae. Proc Natl Acad Sci U S A 113, 6910-6915 (2016); published online EpubJun 21 (10.1073/pnas.1523824113).

2. T. Makushok, P. Alves, S. M. Huisman, A. R. Kijowski, D. Brunner, Sterol-Rich Membrane Domains Define Fission Yeast Cell Polarity. Cell 165, 1182-1196 (2016); published online EpubMay 19 (10.1016/j.cell.2016.04.037).

3. N. E. Ziolkowska, R. Christiano, T. C. Walther, Organized living: formation mechanisms and functions of plasma membrane domains in yeast. Trends Cell Biol 22, 151-158 (2012); published online EpubMar (10.1016/j.tcb.2011.12.002).

4. A. Athanasopoulos, B. Andre, V. Sophianopoulou, C. Gournas, Fungal plasma membrane domains. FEMS Microbiol Rev, (2019); published online EpubAug 27 (10.1093/femsre/fuz022).

5. F. Spira, N. S. Mueller, G. Beck, P. von Olshausen, J. Beig, R. Wedlich-Soldner, Patchwork organization of the yeast plasma membrane into numerous coexisting domains. Nat Cell Biol 14, 640-648 (2012); published online EpubApr 29 (10.1038/ncb2487).

6. T. C. Walther, J. H. Brickner, P. S. Aguilar, S. Bernales, C. Pantoja, P. Walter, Eisosomes mark static sites of endocytosis. Nature 439, 998-1003 (2006)10.1038/nature04472).

7. H. X. Wang, L. M. Douglas, P. Vesela, R. Rachel, J. Malinsky, J. B. Konopka, Eisosomes promote the ability of Sur7 to regulate plasma membrane organization in Candida albicans. Mol Biol Cell 27, 1663-1675 (2016); published online EpubMay 15 (10.1091/mbc.E16-01-0065).

8. R. Kabeche, S. Baldissard, J. Hammond, L. Howard, J. B. Moseley, The filament-forming protein Pill assembles linear eisosomes in fission yeast. Mol Biol Cell 22, 4059-4067 (2011); published online EpubNov (10.1091/mbc.E11-07-0605).

9. I. Vangelatos, K. Roumelioti, C. Gournas, T. Suarez, C. Scazzocchio, V. Sophianopoulou, Eisosome organization in the filamentous ascomycete Aspergillus nidulans. Eukaryot Cell 9, 14411454 (2010); published online EpubOct (10.1128/EC.00087-10).

10. L. Karotki, J. T. Huiskonen, C. J. Stefan, N. E. Ziółkowska, R. Roth, M. A. Surma, N. J. Krogan, S. D. Emr, J. Heuser, K. Grünewald, T. C. Walther, Eisosome proteins assemble into a membrane scaffold. The Journal of Cell Biology 195, 889-902 (2011)10.1083/jcb.201104040).

11. A. Olivera-Couto, V. Salzman, M. Mailhos, Michelle A. Digman, E. Gratton, Pablo S. Aguilar, Eisosomes Are Dynamic Plasma Membrane Domains Showing Pil1-Lsp1 Heteroligomer Binding Equilibrium. Biophysical Journal 108, 1633-1644 (2015)10.1016/j.bpj.2015.02.011).

12. G. Z. Luo, M. Costanzo, C. Boone, R. C. Dickson, Nutrients and the Pkh1/2 and Pkc1 Protein Kinases Control mRNA Decay and P-body Assembly in Yeast. Journal of Biological Chemistry 286, 8759-8770 (2011); published online EpubMar 18 (10.1074/jbc.M110.196030).

13. V. Mascaraque, M. L. Hernaez, M. Jimenez-Sanchez, R. Hansen, C. Gil, H. Martin, V. J. Cid, M. Molina, Phosphoproteomic Analysis of Protein Kinase C Signaling in Saccharomyces cerevisiae Reveals Slt2 Mitogen-activated Protein Kinase (MAPK)-dependent Phosphorylation of Eisosome Core Components. Molecular \& Cellular Proteomics 12, 557-574 (2013); published online EpubMar (10.1074/mcp.M112.020438). 
14. M. Inagaki, T. Schmelzle, K. Yamaguchi, K. Irie, M. N. Hall, K. Matsumoto, PDK1 homologs activate the Pkc1-mitogen-activated protein kinase pathway in yeast. Mol Cell Biol 19, 8344-8352 (1999); published online EpubDec (10.1128/mcb.19.12.8344).

15. K. E. Moreira, S. Schuck, B. Schrul, F. Frohlich, J. B. Moseley, T. C. Walther, P. Walter, Seg1 controls eisosome assembly and shape. J Cell Biol 198, 405-420 (2012); published online EpubAug $6(10.1083 /$ jcb.201202097).

16. T. Grousl, M. Opekarova, V. Stradalova, J. Hasek, J. Malinsky, Evolutionarily conserved 5'-3' exoribonuclease Xrn1 accumulates at plasma membrane-associated eisosomes in post-diauxic yeast. PLoS One 10, e0122770 (2015)10.1371/journal.pone.0122770).

17. C. Gournas, S. Gkionis, M. Carquin, L. Twyffels, D. Tyteca, B. Andre, Conformation-dependent partitioning of yeast nutrient transporters into starvation-protective membrane domains. Proc Natl Acad Sci U S A 115, E3145-E3154 (2018); published online EpubApr 3 (10.1073/pnas.1719462115).

18. A. Moharir, L. Gay, D. Appadurai, J. Keener, M. Babst, Eisosomes are metabolically regulated storage compartments for APC-type nutrient transporters. Mol Biol Cell 29, 2113-2127 (2018); published online EpubAug 15 (10.1091/mbc.E17-11-0691).

19. J. E. Foderaro, L. M. Douglas, J. B. Konopka, MCC/Eisosomes Regulate Cell Wall Synthesis and Stress Responses in Fungi. J Fungi (Basel) 3, (2017); published online EpubNov 3 (10.3390/jof3040061).

20. R. Kabeche, L. Howard, J. B. Moseley, Eisosomes provide membrane reservoirs for rapid expansion of the yeast plasma membrane. Journal of Cell Science 128, 4057-4062 (2015)10.1242/jcs.176867).

21. M. E. Young, T. S. Karpova, B. Brugger, D. M. Moschenross, G. K. Wang, R. Schneiter, F. T. Wieland, J. A. Cooper, The Sur7p family defines novel cortical domains in Saccharomyces cerevisiae, affects sphingolipid metabolism, and is involved in sporulation. Mol Cell Biol 22, $927-$ 934 (2002); published online EpubFeb (10.1128/mcb.22.3.927-934.2002).

22. M. Babst, Eisosomes at the intersection of TORC1 and TORC2 regulation. Traffic 20, 543-551 (2019); published online EpubAug (10.1111/tra.12651).

23. J. Zahumensky, J. Malinsky, Role of MCC/Eisosome in Fungal Lipid Homeostasis. Biomolecules 9, (2019); published online EpubJul 25 (10.3390/biom9080305).

24. D. Berchtold, M. Piccolis, N. Chiaruttini, I. Riezman, H. Riezman, A. Roux, T. C. Walther, R. Loewith, Plasma membrane stress induces relocalization of Slm proteins and activation of TORC2 to promote sphingolipid synthesis. Nat Cell Biol 14, 542-547 (2012); published online EpubApr 15 $(10.1038 / \mathrm{ncb} 2480)$.

25. N. Kedersha, P. Ivanov, P. Anderson, Stress granules and cell signaling: more than just a passing phase? Trends Biochem Sci 38, 494-506 (2013); published online EpubOct (10.1016/j.tibs.2013.07.004).

26. N. Kedersha, G. Stoecklin, M. Ayodele, P. Yacono, J. Lykke-Andersen, M. J. Fritzler, D. Scheuner, R. J. Kaufman, D. E. Golan, P. Anderson, Stress granules and processing bodies are dynamically linked sites of mRNP remodeling. J Cell Biol 169, 871-884 (2005); published online EpubJun 20 (10.1083/jcb.200502088). 
27. A. Khong, T. Matheny, S. Jain, S. F. Mitchell, J. R. Wheeler, R. Parker, The Stress Granule Transcriptome Reveals Principles of mRNA Accumulation in Stress Granules. Mol Cell 68, 808820 e805 (2017); published online EpubNov 16 (10.1016/j.molcel.2017.10.015).

28. D. Kaganovich, There Is an Inclusion for That: Material Properties of Protein Granules Provide a Platform for Building Diverse Cellular Functions. Trends Biochem Sci 42, 765-776 (2017); published online EpubOct (10.1016/j.tibs.2017.08.002).

29. T. Amen, D. Kaganovich, Dynamic droplets: the role of cytoplasmic inclusions in stress, function, and disease. Cell Mol Life Sci 72, 401-415 (2015); published online EpubFeb (10.1007/s00018014-1740-y).

30. P. Anderson, N. Kedersha, Stress granules: the Tao of RNA triage. Trends in Biochemical Sciences 33, 141-150 (2008)10.1016/j.tibs.2007.12.003).

31. D. S. W. Protter, R. Parker, Principles and Properties of Stress Granules. Trends in Cell Biology 26, 668-679 (2016)10.1016/j.tcb.2016.05.004).

32. J. R. Buchan, J. H. Yoon, R. Parker, Stress-specific composition, assembly and kinetics of stress granules in Saccharomyces cerevisiae. J Cell Sci 124, 228-239 (2011); published online EpubJan 15 (10.1242/jcs.078444).

33. T. Kobayashi, S. Winslow, L. Sunesson, U. Hellman, C. Larsson, PKCalpha binds G3BP2 and regulates stress granule formation following cellular stress. PLoS One 7, e35820 (2012)10.1371/journal.pone.0035820).

34. A. P. Sfakianos, L. E. Mellor, Y. F. Pang, P. Kritsiligkou, H. Needs, H. Abou-Hamdan, L. Desaubry, G. B. Poulin, M. P. Ashe, A. J. Whitmarsh, The mTOR-S6 kinase pathway promotes stress granule assembly. Cell Death Differ 25, 1766-1780 (2018); published online EpubNov (10.1038/s41418-018-0076-9).

35. K. Thedieck, B. Holzwarth, M. T. Prentzell, C. Boehlke, K. Klasener, S. Ruf, A. G. Sonntag, L. Maerz, S. N. Grellscheid, E. Kremmer, R. Nitschke, E. W. Kuehn, J. W. Jonker, A. K. Groen, M. Reth, M. N. Hall, R. Baumeister, Inhibition of mTORC1 by astrin and stress granules prevents apoptosis in cancer cells. Cell 154, 859-874 (2013); published online EpubAug 15 (10.1016/j.cell.2013.07.031).

36. K. J. Roux, D. I. Kim, M. Raida, B. Burke, A promiscuous biotin ligase fusion protein identifies proximal and interacting proteins in mammalian cells. The Journal of Cell Biology 196, 801-810 (2012)10.1083/jcb.201112098).

37. T. C. Walther, P. S. Aguilar, F. Frohlich, F. Chu, K. Moreira, A. L. Burlingame, P. Walter, Pkhkinases control eisosome assembly and organization. EMBO J 26, 4946-4955 (2007); published online EpubDec 12 (7601933 [pii]

10.1038/sj.emboj.7601933).

38. G. Luo, A. Gruhler, Y. Liu, O. N. Jensen, R. C. Dickson, The sphingolipid long-chain base-Pkh1/2Ypk1/2 signaling pathway regulates eisosome assembly and turnover. J Biol Chem 283, 10433 10444 (2008); published online EpubApr 18 (10.1074/jbc.M709972200).

39. P. J. Kahle, T. Kobayashi, S. Winslow, L. Sunesson, U. Hellman, C. Larsson, PKC $\alpha$ Binds G3BP2 and Regulates Stress Granule Formation Following Cellular Stress. Plos One 7, e35820 (2012)10.1371/journal.pone.0035820). 
40. I. Sagot, B. Pinson, B. Salin, B. Daignan-Fornier, Actin bodies in yeast quiescent cells: an immediately available actin reserve? Mol Biol Cell 17, 4645-4655 (2006); published online EpubNov (10.1091/mbc.e06-04-0282).

41. S. Kroschwald, S. Maharana, D. Mateju, L. Malinovska, E. Nuske, I. Poser, D. Richter, S. Alberti, Promiscuous interactions and protein disaggregases determine the material state of stress-inducible RNP granules. eLife 4, e06807 (2015); published online EpubAug 04 (10.7554/eLife.06807).

42. D. Mateju, T. M. Franzmann, A. Patel, A. Kopach, E. E. Boczek, S. Maharana, H. O. Lee, S. Carra, A. A. Hyman, S. Alberti, An aberrant phase transition of stress granules triggered by misfolded protein and prevented by chaperone function. EMBO J 36, 1669-1687 (2017); published online EpubJun 14 (10.15252/embj.201695957).

43. M. R. Heider, M. Gu, C. M. Duffy, A. M. Mirza, L. L. Marcotte, A. C. Walls, N. Farrall, Z. Hakhverdyan, M. C. Field, M. P. Rout, A. Frost, M. Munson, Subunit connectivity, assembly determinants and architecture of the yeast exocyst complex. Nature Structural \& Molecular Biology 23, 59-66 (2015)10.1038/nsmb.3146).

44. H. Nonaka, K. Tanaka, H. Hirano, T. Fujiwara, H. Kohno, M. Umikawa, A. Mino, Y. Takai, A downstream target of RHO1 small GTP-binding protein is PKC1, a homolog of protein kinase C, which leads to activation of the MAP kinase cascade in Saccharomyces cerevisiae. EMBO J 14, 5931-5938 (1995); published online EpubDec 1 (

45. A. J. Lam, F. St-Pierre, Y. Gong, J. D. Marshall, P. J. Cranfill, M. A. Baird, M. R. McKeown, J. Wiedenmann, M. W. Davidson, M. J. Schnitzer, R. Y. Tsien, M. Z. Lin, Improving FRET dynamic range with bright green and red fluorescent proteins. Nat Methods 9, 1005-1012 (2012); published online EpubOct (10.1038/nmeth.2171).

46. C. Reinoso-Martin, C. Schuller, M. Schuetzer-Muehlbauer, K. Kuchler, The yeast protein kinase $\mathrm{C}$ cell integrity pathway mediates tolerance to the antifungal drug caspofungin through activation of Slt2p mitogen-activated protein kinase signaling. Eukaryot Cell 2, 1200-1210 (2003); published online EpubDec (10.1128/ec.2.6.1200-1210.2003).

47. V. Denis, M. S. Cyert, Molecular analysis reveals localization of Saccharomyces cerevisiae protein kinase $\mathrm{C}$ to sites of polarized growth and Pkclp targeting to the nucleus and mitotic spindle. Eukaryot Cell 4, 36-45 (2005); published online EpubJan (10.1128/EC.4.1.36-45.2005).

48. B. S. Kang, O. G. French, J. J. Sando, C. S. Hahn, Activation-dependent degradation of protein kinase C eta. Oncogene 19, 4263-4272 (2000); published online EpubAug 31 (10.1038/sj.onc.1203779).

49. J. J. Heinisch, R. Rodicio, Protein kinase $\mathrm{C}$ in fungi-more than just cell wall integrity. FEMS Microbiol Rev 42, (2018); published online EpubJan 1 (10.1093/femsre/fux051).

50. L. M. Douglas, J. B. Konopka, Plasma membrane architecture protects Candida albicans from killing by copper. PLoS Genet 15, e1007911 (2019); published online EpubJan (10.1371/journal.pgen.1007911).

51. F. Sherman, Getting started with yeast. Methods Enzymol 350, 3-41 (2002)10.1016/s00766879(02)50954-x).

52. B. J. Thomas, R. Rothstein, Elevated recombination rates in transcriptionally active DNA. Cell 56, 619-630 (1989); published online EpubFeb 24 (10.1016/0092-8674(89)90584-9).

53. C. B. Brachmann, A. Davies, G. J. Cost, E. Caputo, J. Li, P. Hieter, J. D. Boeke, Designer deletion strains derived from Saccharomyces cerevisiae S288C: a useful set of strains and plasmids for PCR- 
mediated gene disruption and other applications. Yeast 14, 115-132 (1998); published online EpubJan 30 (10.1002/(SICI)1097-0061(19980130)14:2<115::AID-YEA204>3.0.CO;2-2).

54. A. Baudin, O. Ozier-Kalogeropoulos, A. Denouel, F. Lacroute, C. Cullin, A simple and efficient method for direct gene deletion in Saccharomyces cerevisiae. Nucleic Acids Res 21, 3329-3330 (1993); published online EpubJul 11 (10.1093/nar/21.14.3329).

55. R. D. Gietz, R. H. Schiestl, A. R. Willems, R. A. Woods, Studies on the transformation of intact yeast cells by the LiAc/SS-DNA/PEG procedure. Yeast 11, 355-360 (1995); published online EpubApr 15 (10.1002/yea.320110408).

56. T. Amen, D. Kaganovich, Integrative modules for efficient genome engineering in yeast. Microb Cell 4, 182-190 (2017); published online EpubJun 5 (10.15698/mic2017.06.576).

57. M. A. Sheff, K. S. Thorn, Optimized cassettes for fluorescent protein tagging in Saccharomyces cerevisiae. Yeast 21, 661-670 (2004); published online EpubJun (10.1002/yea.1130).

58. J. Rappsilber, M. Mann, Y. Ishihama, Protocol for micro-purification, enrichment, prefractionation and storage of peptides for proteomics using StageTips. Nat Protoc 2, 1896-1906 (2007)10.1038/nprot.2007.261).

59. J. Cox, M. Mann, MaxQuant enables high peptide identification rates, individualized p.p.b.-range mass accuracies and proteome-wide protein quantification. Nat Biotechnol 26, 1367-1372 (2008); published online EpubDec (10.1038/nbt.1511).

60. J. Cox, M. Y. Hein, C. A. Luber, I. Paron, N. Nagaraj, M. Mann, Accurate proteome-wide labelfree quantification by delayed normalization and maximal peptide ratio extraction, termed MaxLFQ. Mol Cell Proteomics 13, 2513-2526 (2014); published online EpubSep (10.1074/mcp.M113.031591).

61. J. Montojo, K. Zuberi, H. Rodriguez, F. Kazi, G. Wright, S. L. Donaldson, Q. Morris, G. D. Bader, GeneMANIA Cytoscape plugin: fast gene function predictions on the desktop. Bioinformatics 26, 2927-2928 (2010); published online EpubNov 15 (10.1093/bioinformatics/btq562).

62. M. K. Sung, W. K. Huh, Bimolecular fluorescence complementation analysis system for in vivo detection of protein-protein interaction in Saccharomyces cerevisiae. Yeast 24, 767-775 (2007); published online EpubSep (10.1002/yea.1504).

63. M. R. Heider, M. Gu, C. M. Duffy, A. M. Mirza, L. L. Marcotte, A. C. Walls, N. Farrall, Z. Hakhverdyan, M. C. Field, M. P. Rout, A. Frost, M. Munson, Subunit connectivity, assembly determinants and architecture of the yeast exocyst complex. Nat Struct Mol Biol 23, 59-66 (2016); published online EpubJan (10.1038/nsmb.3146).

64. J. C. Wootton, Non-globular domains in protein sequences: automated segmentation using complexity measures. Comput Chem 18, 269-285 (1994); published online EpubSep (

65. Z. Dosztanyi, V. Csizmok, P. Tompa, I. Simon, IUPred: web server for the prediction of intrinsically unstructured regions of proteins based on estimated energy content. Bioinformatics 21, 3433-3434 (2005); published online EpubAug 15 (10.1093/bioinformatics/bti541).

66. Y. Perez-Riverol, A. Csordas, J. Bai, M. Bernal-Llinares, S. Hewapathirana, D. J. Kundu, A. Inuganti, J. Griss, G. Mayer, M. Eisenacher, E. Perez, J. Uszkoreit, J. Pfeuffer, T. Sachsenberg, S. Yilmaz, S. Tiwary, J. Cox, E. Audain, M. Walzer, A. F. Jarnuczak, T. Ternent, A. Brazma, J. A. Vizcaino, The PRIDE database and related tools and resources in 2019: improving support for quantification data. Nucleic Acids Res 47, D442-D450 (2019); published online EpubJan 8 (10.1093/nar/gky1106). 
Chapter 5

Conclusion 


\section{Conclusion}

Cellular metabolism has a remarkable ability to successfully navigate two opposing objectives: robustness and adaptability. Robustness means standing unperturbed in the face of constant minor fluctuations in the environment, while successful adaptation requires that certain fluctuations trigger a massive set of systemic changes (Masel and Trotter, 2010; Stelling et al., 2004). Cells excel at robustness by compartmentalizing biochemical pathways in distinct organelles (Gottschling and Nystrom, 2017; Moldavski et al., 2015) and membraneless subdomains (Amen and Kaganovich, 2015; An et al., 2008; Kaganovich, 2017; Kaganovich et al., 2008; Maharana et al., 2018), creating a nested dynamic network regulating cellular functioning (He et al., 2013; Hunter, 2009; Smart et al., 2008). The stability of this regulatory network is seemingly at cross purposes with adaptation to severe and unprecedented stress. Adaptation requires sensitivity to only those challenges that call for a partial breakdown of an existing metabolic network, and its replacement with a different one (Schreier et al., 2017). Moreover, metabolic flexibility and remodeling require communication between metabolic modules that otherwise function independently (Muoio, 2014). Fine-tuned metabolic flexibility is particularly essential during starvation stress, when parts of the metabolic infrastructure have to be uprooted and replaced in response to changing fuel sources, before ATP shortages prove fatal (Gomes et al., 1985; Leprivier et al., 2013). In order for cells to survive extreme fluctuations in fuel sources, they must solve the multidimensional problem of balancing energy production with the rationing of scarce resources and the damage caused by metabolic activity. The data presented in this thesis argue that membraneless organelles, Stress Granules (SGs), are master regulators of cellular adaptation to stress, providing robustness of functioning in nutrient starvation. SGs house protein kinase $\mathrm{C}$, preserving it throughout the stress conditions, which allows cells to restart division when normal conditions resume. That reduces the impact of starvation on cellular growth. SGs regulate stress induced mitochondrial fatty acid oxidation response, by channeling lipids away from mitochondria in the long-term starvation. Additionally, SGs favor translation of lipid metabolism associated targets, including lipid droplet proteins, which results in lipid droplet formation.

Together the findings in this thesis show that SGs can exert several modes of actions proposed for membraneless compartments (Kaganovich, 2017). SGs can store enzymes, providing active pool of protein kinase $\mathrm{C}$ to resume division. SGs can filter the PPAR translation signal, by 
and earlier proposed mechanism of retaining longer transcripts (Khong et al., 2017). The regulation of lipid metabolism in the long-term starvation implies that SGs sense cellular metabolic state. Strikingly, genetic or pharmacological perturbation in the assembly of SGs led to an inability to regulate fatty acid oxidation in the long-term starvation, which, in turn, resulted in oxidative damage. In yeast $S$. cerevisiae, inability to form SGs led to a stress-specific defects in growth rates. Thus, SG formation is a general adaptive response to stress.

Aberrant aggregation of SG-resident proteins is implicated in human neurodegenerative conditions, such as Amyotrophic Lateral Sclerosis (ALS) (Arai et al., 2006; Kaganovich, 2017; Lenzi et al., 2015; Li et al., 2013; Mackenzie et al., 2017; Mateju et al., 2017; Patel et al., 2015). Both the inability to form SGs, and persistent formation of overly aggregated SGs lead to a pathological state (Aulas and Vande Velde, 2015; Dewey et al., 2012; Kaganovich, 2017; Khalfallah et al., 2018; Mann et al., 2019; Patel et al., 2015). Our data on SG metabolic regulation help reconcile many cellular and clinical observations pertaining to metabolic dysfunction in ALS (Tefera and Borges, 2017). Metabolic shifts often underlie cellular activity (Knobloch et al., 2017). We show that the inability to mount a functional SG response leads to a breakdown in the control of metabolic outputs. We argue that it contributes to a metabolic dysfunction observed in the disease pathology (Szelechowski et al., 2018; Tefera and Borges, 2017). This suggests that regulating the function of SGs in metabolic output control can be exploited for drug discovery. We identify small molecules that induce and prevent SG formation. We show that molecules inducing SG formation lead to a reduction in fatty acid oxidation, while inhibiting SG formation increases oxidation. Additionally, our data suggest that it is possible that SG pathology involves both the inability to mount a SG response on time and the subsequent persistent accumulation of aggregates as a result of pathological composition. In such case it wouldn't suffice to simply prevent aberrant SG formation. As we demonstrated, SGs exert a set of adaptive functions, that help cells withstand stress conditions. Rather a complex therapy, including replacement of SG adaptive functions would be necessary.

In this thesis I attempt to shift the perception of protein aggregation from a process that is perceived to be dysfunctional to a necessary component of cellular stress adaptation, which exerts many protective functions. The results described here provide a basis for further inquiry into the mechanisms of ALS pathology. It is possible that we can restore SG function and reverse the 
pathology resulting from aberrant aggregation. Since SGs have different functional modes, studying SGs in disease-relevant cell types, such as motor neurons, and especially their terminals forming neuro-muscular junctions, will be an invaluable next step in understanding the mechanisms of neurodegeneration. 


\section{References}

Amen, T., and Kaganovich, D. (2015). Dynamic droplets: the role of cytoplasmic inclusions in stress, function, and disease. Cell Mol Life Sci 72, 401-415.

An, S., Kumar, R., Sheets, E.D., and Benkovic, S.J. (2008). Reversible compartmentalization of de novo purine biosynthetic complexes in living cells. Science 320, 103-106.

Arai, T., Hasegawa, M., Akiyama, H., Ikeda, K., Nonaka, T., Mori, H., Mann, D., Tsuchiya, K., Yoshida, M., Hashizume, Y., et al. (2006). TDP-43 is a component of ubiquitin-positive taunegative inclusions in frontotemporal lobar degeneration and amyotrophic lateral sclerosis. Biochem Biophys Res Commun 351, 602-611.

Aulas, A., and Vande Velde, C. (2015). Alterations in stress granule dynamics driven by TDP-43 and FUS: a link to pathological inclusions in ALS? Front Cell Neurosci 9, 423.

Dewey, C.M., Cenik, B., Sephton, C.F., Johnson, B.A., Herz, J., and Yu, G. (2012). TDP-43 aggregation in neurodegeneration: Are stress granules the key? Brain Research 1462, 16-25.

Gomes, M.I., Kim, W.J., Lively, M.K., and Amos, H. (1985). Heat-shock treatment lethal for mammalian cells deprived of glucose and glutamine: protection by alpha-keto acids. Biochem Biophys Res Commun 131, 1013-1019.

Gottschling, D.E., and Nystrom, T. (2017). The Upsides and Downsides of Organelle Interconnectivity. Cell 169, 24-34.

He, F., Fromion, V., and Westerhoff, H.V. (2013). (Im)Perfect robustness and adaptation of metabolic networks subject to metabolic and gene-expression regulation: marrying control engineering with metabolic control analysis. BMC Syst Biol 7, 131.

Hunter, P. (2009). Robust yet flexible. In biological systems, resistance to change and innovation in the light of it go hand in hand. EMBO Rep 10, 949-952.

Kaganovich, D. (2017). There Is an Inclusion for That: Material Properties of Protein Granules Provide a Platform for Building Diverse Cellular Functions. Trends Biochem Sci 42, 765-776.

Kaganovich, D., Kopito, R., and Frydman, J. (2008). Misfolded proteins partition between two distinct quality control compartments. Nature 454, 1088-1095.

Khalfallah, Y., Kuta, R., Grasmuck, C., Prat, A., Durham, H.D., and Vande Velde, C. (2018). TDP-43 regulation of stress granule dynamics in neurodegenerative disease-relevant cell types. Sci Rep 8, 7551.

Khong, A., Matheny, T., Jain, S., Mitchell, S.F., Wheeler, J.R., and Parker, R. (2017). The Stress Granule Transcriptome Reveals Principles of mRNA Accumulation in Stress Granules. Mol Cell 68, 808-820 e805.

Knobloch, M., Pilz, G.A., Ghesquiere, B., Kovacs, W.J., Wegleiter, T., Moore, D.L., Hruzova, M., Zamboni, N., Carmeliet, P., and Jessberger, S. (2017). A Fatty Acid Oxidation-Dependent Metabolic Shift Regulates Adult Neural Stem Cell Activity. Cell Rep 20, 2144-2155.

Lenzi, J., De Santis, R., de Turris, V., Morlando, M., Laneve, P., Calvo, A., Caliendo, V., Chio, A., Rosa, A., and Bozzoni, I. (2015). ALS mutant FUS proteins are recruited into stress granules in induced pluripotent stem cell-derived motoneurons. Disease Models \& Mechanisms 8, 755-766. Leprivier, G., Remke, M., Rotblat, B., Dubuc, A., Mateo, A.R., Kool, M., Agnihotri, S., El-Naggar, A., Yu, B., Somasekharan, S.P., et al. (2013). The eEF2 kinase confers resistance to nutrient deprivation by blocking translation elongation. Cell 153, 1064-1079.

Li, Y.R., King, O.D., Shorter, J., and Gitler, A.D. (2013). Stress granules as crucibles of ALS pathogenesis. J Cell Biol 201, 361-372. 
Mackenzie, I.R., Nicholson, A.M., Sarkar, M., Messing, J., Purice, M.D., Pottier, C., Annu, K., Baker, M., Perkerson, R.B., Kurti, A., et al. (2017). TIA1 Mutations in Amyotrophic Lateral Sclerosis and Frontotemporal Dementia Promote Phase Separation and Alter Stress Granule Dynamics. Neuron 95, 808-816 e809.

Maharana, S., Wang, J., Papadopoulos, D.K., Richter, D., Pozniakovsky, A., Poser, I., Bickle, M., Rizk, S., Guillen-Boixet, J., Franzmann, T.M., et al. (2018). RNA buffers the phase separation behavior of prion-like RNA binding proteins. Science 360, 918-921.

Mann, J.R., Gleixner, A.M., Mauna, J.C., Gomes, E., DeChellis-Marks, M.R., Needham, P.G., Copley, K.E., Hurtle, B., Portz, B., Pyles, N.J., et al. (2019). RNA Binding Antagonizes Neurotoxic Phase Transitions of TDP-43. Neuron 102, 321-338 e328.

Masel, J., and Trotter, M.V. (2010). Robustness and evolvability. Trends Genet 26, 406-414.

Mateju, D., Franzmann, T.M., Patel, A., Kopach, A., Boczek, E.E., Maharana, S., Lee, H.O., Carra, S., Hyman, A.A., and Alberti, S. (2017). An aberrant phase transition of stress granules triggered by misfolded protein and prevented by chaperone function. EMBO J 36, 1669-1687.

Moldavski, O., Amen, T., Levin-Zaidman, S., Eisenstein, M., Rogachev, I., Brandis, A., Kaganovich, D., and Schuldiner, M. (2015). Lipid Droplets Are Essential for Efficient Clearance of Cytosolic Inclusion Bodies. Dev Cell 33, 603-610.

Muoio, D.M. (2014). Metabolic inflexibility: when mitochondrial indecision leads to metabolic gridlock. Cell 159, 1253-1262.

Patel, A., Lee, H.O., Jawerth, L., Maharana, S., Jahnel, M., Hein, M.Y., Stoynov, S., Mahamid, J., Saha, S., Franzmann, T.M., et al. (2015). A Liquid-to-Solid Phase Transition of the ALS Protein FUS Accelerated by Disease Mutation. Cell 162, 1066-1077.

Schreier, H.I., Soen, Y., and Brenner, N. (2017). Exploratory adaptation in large random networks. Nat Commun 8, 14826.

Smart, A.G., Amaral, L.A., and Ottino, J.M. (2008). Cascading failure and robustness in metabolic networks. Proc Natl Acad Sci U S A 105, 13223-13228.

Stelling, J., Sauer, U., Szallasi, Z., Doyle, F.J., 3rd, and Doyle, J. (2004). Robustness of cellular functions. Cell 118, 675-685.

Szelechowski, M., Amoedo, N., Obre, E., Leger, C., Allard, L., Bonneu, M., Claverol, S., Lacombe, D., Oliet, S., Chevallier, S., et al. (2018). Metabolic Reprogramming in Amyotrophic Lateral Sclerosis. Sci Rep-Uk 8.

Tefera, T.W., and Borges, K. (2017). Metabolic Dysfunctions in Amyotrophic Lateral Sclerosis Pathogenesis and Potential Metabolic Treatments. Frontiers in Neuroscience 10. 